\title{
Self-assembled InAs/InP quantum dots and quantum dashes: Material structures and devices
}

\author{
Mohammed Zahed Mustafa Khan, Tien Khee Ng, and Boon S. Ooi* \\ Photonics Laboratory, King Abdullah University of Science \& Technology (KAUST), \\ Thu wal 23955-6900, Kingdom of Saudi Arabia \\ *email: boon.ooi@kaust.edu.sa
}

\begin{abstract}
The advances in lasers, electronic and photonic integrated circuits (EPIC), optical interconnects as well as the modulation techniques allow the present day society to embrace the convenience of broadband, high speed internet and mobile network connectivity. However, the steep increase in energy demand and bandwidth requirement calls for further innovation in ultra-compact EPIC technologies. In the optical domain, innovation in the laser technologies beyond the current quantum well (Qwell) based laser technologies are already taking place and presenting very promising results. Homogeneously grown quantum dot (Qdot) lasers, can serve in the future energy saving information and communication technologies (ICT) as the work-horse for transmitting information through optical fiber. The encouraging results in the zero-dimensional (0D) structures emitting at $980 \mathrm{~nm}$, in the form of vertical cavity surface emitting laser (VCSEL), are already operational at low threshold current density and capable of $40 \mathrm{Gbps}$ error-free transmission at $108 \mathrm{fJ} / \mathrm{bit}$. Eventual achievements for lasers operating in the $\mathrm{O}_{-}, \mathrm{C}-, \mathrm{L}-, \mathrm{U}-$ bands, and beyond will eventually lay the foundation for green ICT. On the hand, the inhomogeneously grown quasi OD quantum dash (Qdash) lasers are brilliant solutions for potential broadband connectivity in server farms or access network. A single broadband Qdash laser operating in the stimulated emission mode can replace tens of discrete narrow-band lasers in dense wavelength division multiplexing (DWDM) transmission thereby further saving energy, cost and footprint. In this article, we review the progress of both Qdots and Qdash devices based on the InAs/InGaAlAs/InP and In As/InGaAsP/InP material systems, from the angles of growth and device performance.
\end{abstract}




\section{Contents}

1. Introduction

2. InAs Qdots grown on InP

2.1 InAs/InGaAlAs Material System

2.1.1 Qdots on (311)B InP substrate

2.1.2 Qdots on (100) InP substrate

2.2 InAs/InGaAsP Material System

2.2.1 Qdots on (100) InP substrate

2.2.2 Qdots on (311)B InP substrate

2.3 Post-growth tuning of InAs/InP Qdots

2.4 Towards $>2.0 \mu \mathrm{m}$ wa velength emission

3. InAs/InP Qdots Devices

3.1 InAs/InP Qdots lasers

3.1.1 Lasers on (311)B InP substrate

3.1.2 Lasers on (100) InP substrate

3.1.3 Multiwavelength Qdots lasers

3.1.4 Single mode lasers

3.2 InAs/InP Qdots mode-locked lasers

3.2.1 Two section

3.2.2 Self-pulsation

3.3 InAs/InP Qdots semiconductor optical amplifiers

3.3.1 Broad gain

3.3.2. Ultrafast gain and index dynamics

3.3.3. High speed amplification and signal processing

3.4 InAs/InP Qdots superluminescent diodes

4. InAs Qdashes grown on InP

4.1 InAs/InGaAlAs Material System

4.1.1 Qdashes on (100) InP substrate

4.1.2 Qdash Optical Properties

4.2 InAs/InGaAsP Material System

4.1.1 Qdashes on (100) InP substrate

4.1.2 Qdash Optical Properties

4.3 Post-growth tuning of InAs/InP Qdashes

4.4 Chirped Qdash active region design

5. InAs/InP Qdash Devices

5.1 InAs/InP Qdash lasers

5.1.1 Lasers on (100) InP substrate

5.1.2 Multiwavelength Qdash lasers

5.1.3 Broadband Qdash lasers

5.1.4 Single mode lasers

5.1.5 Injection locking/critical feedback of Qdash lasers

5.2 InAs/InP Qdash mode-locked lasers

5.2.1 Two section

5.2.2 Self-pulsation

5.2.3 Selected system-level applications

5.3 InAs/InP Qdash semiconductor optical a mplifiers

5.3.1 Broad gain

5.3.2. Ultrafast gain and index dynamics

5.3.3. High speed amplification and signal processing

5.4 InAs/InP Qdash superluminescent diodes

6. Conclusion 


\section{Introduction}

Demand for high performance active optical elements in the form of semiconductor lasers, lightemitting diodes, mode-locked lasers, and semiconductor optical amplifiers (SOA), modulators, etc. are significantly rising because of the current demand for large bandwidths in the $1.55 \mu \mathrm{m} \mathrm{C}$-band optical communication window to meet the requirements for the exponentially growing internet and mobile connectivity market. The intervention of optical fiber technology and related active optical elements in the short-reach communications, such as intra-rack and inter/intra-chip optical interconnects, due to the struggling electrical interconnects to satisfy the requirements of bandwidth, packing density, cost, and power consumption, demand further investigations into achieving high quality semiconductor material and devices.

In this regard, quantum confined nanostructure based active-regions in the form of Qwells and selfassembled Qdots have played a pivotal role in the achievement of efficient semiconductor devices, thanks to the high quality epitaxial material growth on GaAs substrate emitting at $\sim 1.3 \mu \mathrm{m}$. Exceptional performances from these semiconductor devices based on InAs/GaAs dots has already been achieved and commercialized [1, 2]. However, the inability of the GaAs based material system to reach the long haul optical communication window of $1.55 \mu \mathrm{m}$ and beyond resulting in the dominance of Qwell based lasers grown on InP substrates to take the center stage. Already in the market and serving the optical fiber technology $1.55 \mu \mathrm{m}$ Qwell devices has shown substantial improvement in device characteristics compared to the double heterostructure counterparts. Recently, much attention has been directed to achieve InAs Qdots at long-wavelengths on InP substrate with the aim of further improving the $1.55 \mu \mathrm{m}$ device performances. However the small lattice mismatch and the resulting complex strain distribution resulted in the formation of a new class of selfassembled Qdash nanostructures instead of Qdots. These are elongated dot-like nanostructures emitting between 1.5 and $2.0 \mu \mathrm{m}$ wavelength range and possess interesting mixed characteristics in between Qwell and Qdots [3]. Epitaxial growth of InAs Qdots which require alteration to the growth kinetics in order to avoid Qdash formation has been addressed by various research groups in recent years by employing conventional and miscut (100) InP, and (311) InP substrates, along with various innovations in growth. In As Qdots and Qdashes on both In GaAsP and InGaAlAs material systems have been developed with respectable device performances. However, the significant reduction of size and shape dispersion (large inhomogeneous broadening) of these nanostructures due to selfassemble growth procedure still pose a challenge in achieving high quality epitaxial material and device performance. Currently, the size dispersion, characterized in terms of photoluminescence (PL) linewidth (full-width-at-half-maximum, FWHM), are $\sim 20 \mathrm{meV}$ at $10 \mathrm{~K}$ for Qdots [4] and $\sim 50 \mathrm{meV}$ at room temperature for Qdashes [5-7]. These values indicate further improvement is required to make it as competitive as the matured InAs/GaAs Qdots on GaAs substrate technology [1, 2].

Alternately, by exploiting the inherent inhomogeneous nature and ultra-broad gain profile of InAs/InP Qdots and Qdashes, a highly attractive broadband optical component can be achieved. This will enable broadband SOAs, electro-absorption modulator, photodetector to be implemented as an integral part of the DWDM technologies. An ultrabroad gain profile of $>300 \mathrm{~nm}$ from Qdash active elements, as well as lasing and amplified spontaneous emission (ASE) bandwidths crossing more than $50 \mathrm{~nm}$ and $700 \mathrm{~nm}$, respectively, from lasers and superluminescent diodes (SLD) are two examples of the recent significant developments in this field of study $[5,8,9]$. Broadband nature of the In As/InP Qdot and Qdash laser diodes has been exploited to generate ultra-short femto-second pulses of $158 \mathrm{fs}$ at $2.21 \mathrm{THz}$ based on the passive mode-locked scheme [10,11] which find important applications particularly in high speed optical time-domain multiplexer (OTDM), wavelength division multiplexed (WDM) communications, and optical clocking to replace electronic clocking in high-speed electronic circuits, such as a microprocessor. 

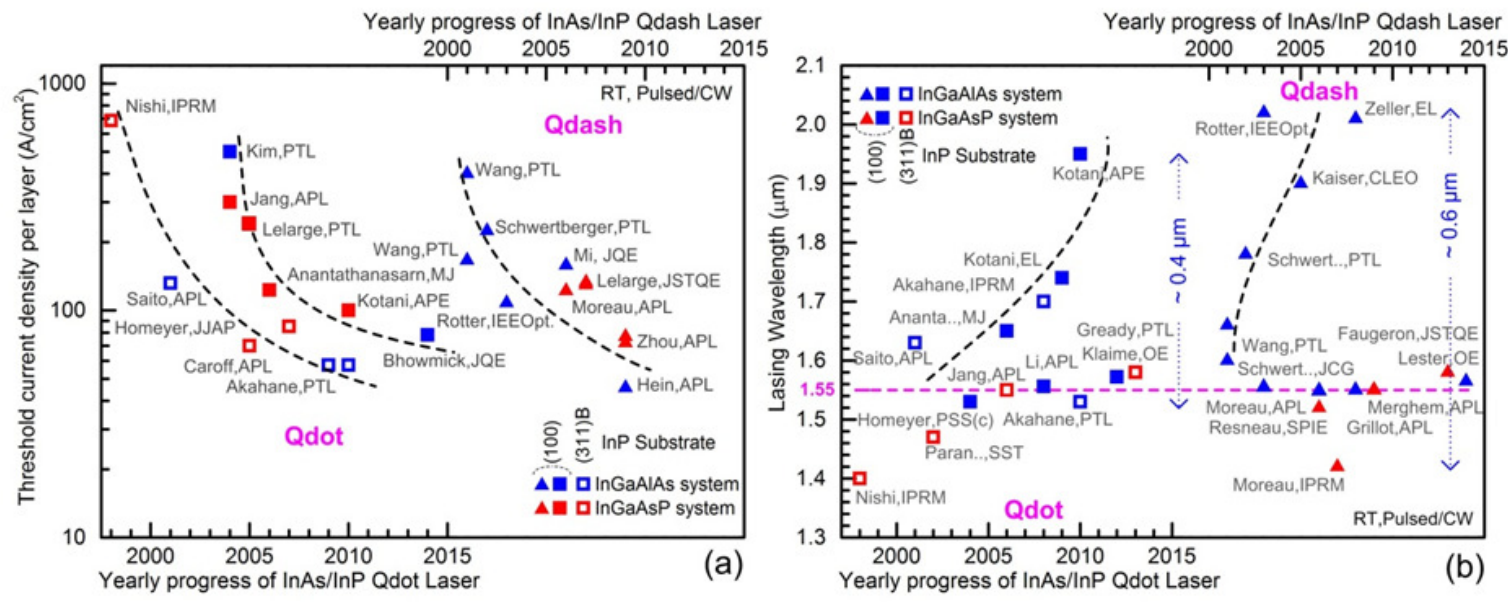

Fig. 1 Chronological progress in the (a) reduction of the threshold current density per layer, and (b) room temperature lasing wavelength tunablity of InAs/InP Qdot (squares) and Qdash (triangles) semiconductor lasers. The dash lines are guide to the eyes showing improvement trend. The data points are collected from the references.

Furthermore, the wavelength tuning capability of InAs/InP Qdot and Qdash devices reaching $2 \mu \mathrm{m}$ and beyond in tandem with their broad gain profile has a potential impact on various multidisciplinary fields. For instance, in medical diagnostics, a broadband semiconductor optical source would enable a compact, high axial resolution, low cost, optical coherent tomography (OCT) system for tissue and bio-molecule imaging, particularly in dentistry and bone-related disease diagnostics [12-15]. In addition, a compact $2 \mu \mathrm{m}$ laser source would be a promising candidate for highly precise surgical application for both soft and hard tissues such as precise tissue ablation, ophthalmic surgery and dentistry, neurosurgery, spinal surgery, etc. where currently solid state lasers dominate [16]. In spectroscopy methods such as absorption, transmission, luminescent, etc., for material and molecular characterizations, a compact long wavelength broadband light source can be employed. These optical sources also has the potential to offer compact hand-held chemical sensor capable of in-situ multicomponent analysis (simultaneous sensing of $\mathrm{CH}_{4}, \mathrm{CO}, \mathrm{CO}_{2}, \mathrm{H}_{2} \mathrm{~S}, \mathrm{HCl}, \mathrm{NH}_{3}, \mathrm{C}_{2} \mathrm{H}_{4}, \mathrm{C}_{2} \mathrm{H}_{2}$, $\mathrm{C}_{2} \mathrm{H}_{6}, \mathrm{C}_{6} \mathrm{H}_{6}$, etc.) as well as integrated real-time near-infrared Lidar (LIght Detection And Ranging) system for monitoring environmental health, and managing emission of greenhouse gases and hazardous chemicals. Many high precision optical metrology systems require light sources emitting broad spectrum of wavelength in the near infrared regime. These include optical time-domain reflectometry (OTDR) for optical fiber fault measurements, and DWDM component characterizations, optical instrumentation, etc., and fiber gyroscopes. Deployment of ultra-broadband optical sources in OTDR would greatly improve the system sensitivity and resolution. In material processing, a compact, high power, near infrared wavelength $(\sim 2 \mu \mathrm{m})$ lasers would allow direct processing of plastics which are transparent in the visible wavelength range via laser cutting, welding, and marking [16]. Besides, long wavelength optical source based OCT systems are finding niche applications in the field of dimensional metrology, material research, non-destructive testing (NDT), for imaging ceramics, studying on the penetration depths in various polymer materials as well as on birefringence imaging of different crystalline polymer structures, are already gaining popularity [17], and could further benefit by achieving larger penetration depth offered by ultrabroadband optical sources.

In a nutshell, the chronological progress of the performance of InAs/InP Qdot and Qdash lasers and mode-locked lasers are plotted in Figs. 1 and 2, respectively. The significant improvement of the threshold current density per Qdots/Qdashes layer of InAs/InP lasers, as illustrated in Fig. 1(a), is indicative of a key step towards highly efficient semiconductor lasers. Although the best values of $\sim 57 \mathrm{~A} / \mathrm{cm}^{2}$ (Qdots) and $\sim 45 \mathrm{~A} / \mathrm{cm}^{2}$ (Qdashes) are far inferior to the InAs/GaAs Qdots material system $\left(17 \mathrm{~A} / \mathrm{cm}^{2}\right)$, the recent solutions to address the complex growth kinetics of dots/dash on InP 

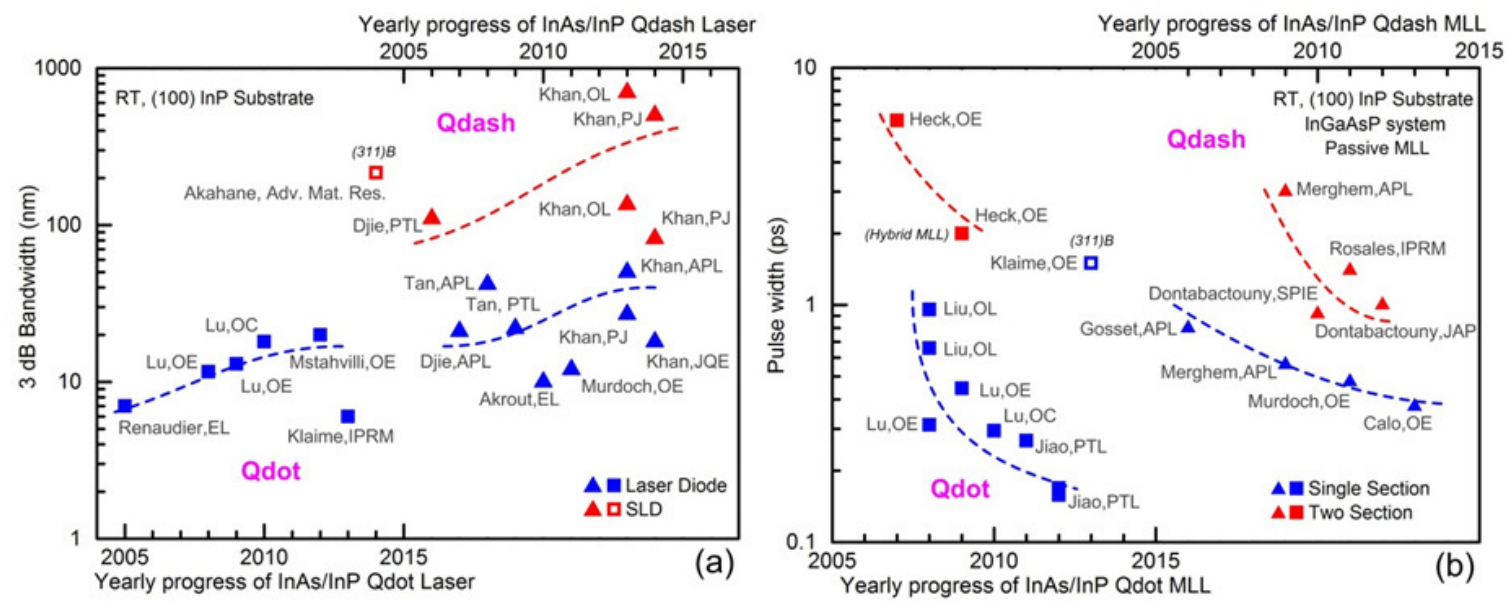

Fig. 2. Chronological progress in (a) achieving ultrabroad emission bandw idth, and (b) reduction in pulse width of InAs/InP Qdot (squares) and Qdash (triangles) lasers and mode-locked lasers, respectively, at room temperature. The dash lines are guide to the eyes showing improvement trend. The data points are collected from the references.

substrate are expected to rapidly reduce threshold current density. The tuning capability of the ground state (GS) lasing wavelength of InAs/InP Qdot and Qdash lasers at room temperature is depicted in Fig. 1(b), with a tuning range of $\sim 0.4 \mu \mathrm{m}$ and $\sim 0.6 \mu \mathrm{m}$, respectively, covering the entire $\mathrm{C}-\mathrm{L}-\mathrm{U}$ optical communication bands. In addition, demonstrations of lasing beyond $2 \mu \mathrm{m}$ wavelength are highly attractive for multi-disciplinary applications as discussed earlier. On the other hand, tapping on the broad gain profile of InAs/InP Qdots and Qdashes, broadband laser diodes and SLDs have been demonstrated and summarized in chronological order in Fig. 2(a). The output power in the range of few hundreds of $\mathrm{mW}$ from laser diodes and tens of $\mathrm{mW}$ from SLDs has been reported. The broadband nature of lasers was the key feature to generate ultra-short femto-second pulses based on the passive mode-locked scheme with reported values progressively reaching few hundred fs range with time, as illustrated in Fig. 2(b). A pulse repetition rate as high as $356 \mathrm{GHz}$ and even reaching terahertz (via external means), and pulse width as low as 158 fs has been achieved on In As/InP Qdot/Qdash material platform, thus showing the potential of this platform for future optical communications and cross-disciplinary applications.

In this review, we examine the existing self-assembled growth of InAs Qdot and Qdash based nanostructures on InP platform for eventual operation in the $\mathrm{C}-\mathrm{L}-\mathrm{U}$ band and towards $2 \mu \mathrm{m}$. The tremendous progress and achievement in this area resulted in an astounding amount of literature. This review took the liberty to divide the work based on Qdots (sections 2, and 3), and Qdashes (sections 4, and 5) on InP substrate. While sections 2 and 4 focus on the achievements in the epitaxial material growth, sections 3 and 5 summarize the respective device implementation and system level performances. The InAs/InGaAlAs and InAs/InGaAsP based active elements discussed in this review are based on (100) and (311)B InP substrates, though it is noted that for III-V-silicon integration for integrated optical telecommunication chip had seen efforts in the growth of Qdots on other unique platforms such as $\mathrm{Ge}$ and $\mathrm{SiGe} / \mathrm{Si}$ based substrates $[18,19]$, as well as other Qdots based on ternary, and Qwell based quintenary material system, i.e. InAsSb and InGaAsNSb, respectively [20]. 


\section{InAs Qdots grown on InP}

The Stranski-Krastanov (SK) [21] growth of InAs Qdots on InP substrate has been a subject of intensive research. One of the main technology hurdles for growing high quality InAs Qdots has been the inclination of elongated wire-like nanostructures formation along the [0-11] crystal directions known as quantum-dashes. The formation of quantum-dashes on InP substrate has been attributed to the lattice mismatch of $3.2 \%$ between In As and InP and the possible complex growth kinetics, growth conditions, buffer layer surface morphology and composition [22, 23]. In addition, another major problem in the growth of InAs Qdots on InP substrate is the relatively large Qdots inhomogeneity compared to the In As/GaAs Qdots system. In the existing literature, various epitaxy growth techniques have been explored by various groups in three key growing systems viz. molecular beam epitaxy (MBE), metal-organic chemical vapor deposition (MOCVD), and chemical beam epitaxy (CBE). Here we will review the achievements in the material growth process of InAs Qdots on InP for two dominant material systems: (i) InAs/InGaAlAs and (ii) InAs/InGaAsP structures.

\subsection{InAs/InGaAlAs Material System}

The In As/InGaAlAs system is preferred due to its large band offset between the InAs dots and the In GaAlAs barrier, eliminating the As/P source exchange during the growth process. It also gives liberty in design of the barriers avoiding complicated quaternary InGaAsP alloys by simply adjusting the relative ratio of $\mathrm{Ga}$ and $\mathrm{Al}$ keeping a constant In flux and avoiding the switching of group $\mathrm{V}$ elements during growth [24]. However, MBE growth of In As on InGaAlAs buffer layer utilizing (100) InP substrate results in elongated nanostructures along the [0-11] direction as shown in Fig. 3(a) has been attributed to the significant influence of the effects of phase separation caused by the different growth behavior of In, Al, and Ga atoms [5, 25, 26]. On the other hand, growth of InAs on high Miller index (311)B InP substrate resulted in high density round shaped Qdots. This is reported by Saito et al. [27] and shown in Fig. 3(b). The Qdots and Qdash areal density are $9 \times 10^{10} \mathrm{~cm}^{-2}$ and $2 \times 10^{10} \mathrm{~cm}^{-2}$, respectively which is advantageous in achieving large optical gain. It is postulated that $\mathrm{Al}$ content in the buffer layer caused the formation of smaller size and higher density InAs Qdots. Moreover, the PL intensity from the single stack InAs Qdot, shown in Fig. 3(c), after capping with InGaAlAs, showed stronger emission from homogeneous Qdots with narrow PL linewidth of 51 meV centered around $1.55 \mu \mathrm{m}$. In the case of Qdash, a broad PL emission and comparatively low in tensity is observed due to highly inhomogeneous nature of the nanostructure. This is undesirable as it leads to laser performance degradation. However, our group exploited this feature of Qdashes in realization of novel devices and has been discussed in section 5. In the following, we will review the systematic progress in the growth of high quality, large density InAs Qdots on different InP substrate orientations.

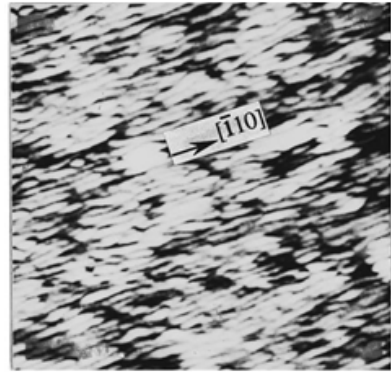

(a)

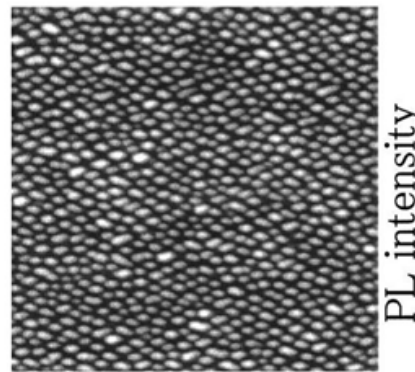

(b)

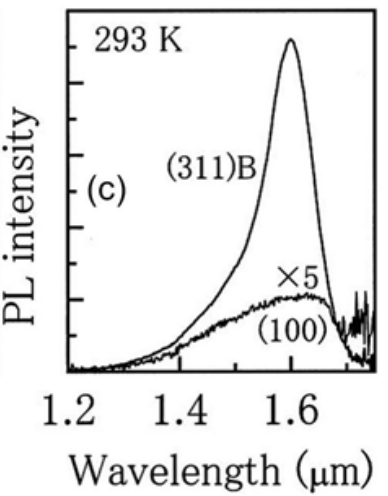

Fig. 3. Atomic force microscopy (AFM) images of InAs Qdots on InGaAlAs buffer on (a) (100) InP and (b) (311)B InP substrate. (b) Room temperature photolumine scence from InAs Q dots on (100) InP and (311)B substrates. Taken from [27, 28]. 


\subsubsection{Qdots on (311)B InP substrate}

The formation of InAs Qdots on (311)B InP substrate was initially reported by Notzel et al. [29] using MOCVD growth technique. Utilizing a thin (3-5 nm) strained InGaAs layer on $100 \mathrm{~nm}$ thick AlInAs buffer layer, a OD microstructure was observed. This growth on high Miller index semiconductor surfaces opened door for growing InAs Qdots on InP substrate for long wavelength near infra-red device applications.
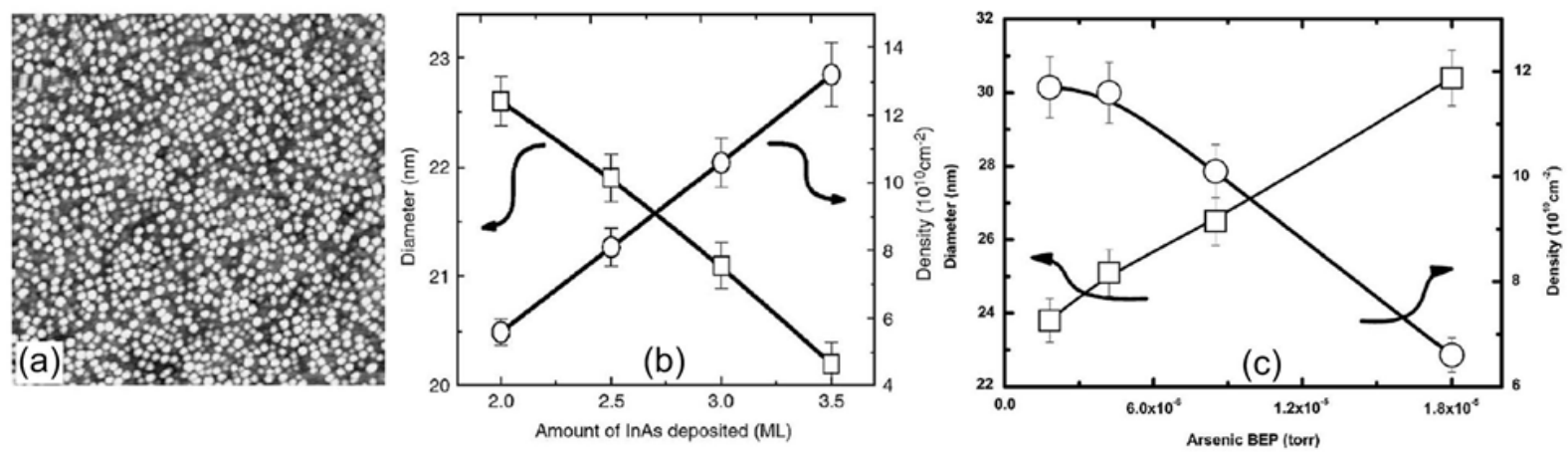

Fig. 4. (a) AFM images ( $\left.1 \times 1 \mu \mathrm{m}^{2}\right)$ of InAs Q dots grown on AlInAs alloy lattice matched to InP, after InAs deposition at 3.5 ML nominal thickness. (b) Qdots diameter and density versus amount of InAs deposited and (c) arsenic beam equivalent pressure (BEP) during the Qdots formation. Adapted from [30].

Following this work, Alghoraibi et al. [30] investigated the formation of Qdots on the highindex (311)B InP substrate via MBE growth. A decrease (increase) in the Qdot diameter (density) was observed for Al-rich surface lattice matched buffer layers $\left(\mathrm{Al}_{0.48} \mathrm{In}_{0.52} \mathrm{As}, \mathrm{Al}_{0.29} \mathrm{Ga}_{0.19} \mathrm{In}_{0.52} \mathrm{As}\right.$, and $\mathrm{In}_{0.53} \mathrm{Ga}_{0.47} \mathrm{As}$ ). Moreover, a continuous increase in Qdot density with the amount of InAs deposition is reported on AlInAs buffer layer, reaching a value as high as $1.3 \times 10^{11} \mathrm{~cm}^{-2}$ with small Qdots $(\sim 20 \mathrm{~nm})$ as shown in the AFM image of Fig. 4(a). The evolution of Qdot density and size, illustrated in Fig. 4(b), is attributed to the interacting stress fields induced by the Qdot within the substrate. On the other hand, a drastic Qdot size reduction (2.2 $\mathrm{nm}$ mean height) and sharp density increase $\left(1.2 \times 10^{11} \mathrm{~cm}^{-2}\right)$ are observed when the As flux [measured in terms of beam equivalent pressure, (BEP)] is reduced during the Qdots formation (Fig. 4(c)). These results are similar to that reported by Caroff et al. using InAs/InGaAsP material system [31], discussed in section 2.2.2. The authors postulated that the increase in the InAs (311) B surface energy at low As pressure, which favors Qdots nucleation, has led to high density of small dots.

Stacking of Qdot layers for increasing the material gain is a standard process employed in quantum-confined nanostructure based active region device designs. However, the tensile strain generated by the InAs Qdots is not compensated as the capping layer is normally lattice matched to the substrate material. This issue has been successfully addressed by Akahane et al. using MBE growth technique who proposed and demonstrated a strain-compensation scheme [32]. The approach was based on adjusting the composition of the InGaAlAs spacer layers to have a smaller lattice constant than that of the InP substrate in order to achieve strain compensation after the growth of one cycle of Qdots and the spacer layer. Fig. 5(a) shows the AFM image of a single layer of InAs Qdots on (311)B InP substrate with average diameter, fluctuation and density of $51.2 \mathrm{~nm}, 13.4 \%$ and $7.84 \times 10^{10} \mathrm{~cm}^{-2}$. The corresponding figures of merits for a equivalent 20 stack Qdots are $54 \mathrm{~nm}$, $10.1 \%$ and $7.36 \mathrm{~cm}^{-2}$, respectively. These impressive results have been attributed to the quaternary spacer layer which allowed continuous change of its lattice constant by controlling the composition, and the $\mathrm{Al}$ in the spacer layer which plays the key role by preventing the segregation of In in In GaAlAs [33]. In a recent demonstration, successful growth of 300 stack Qdots (shown in Fig. 5(b) and (c)) with room temperature PL emission at $\sim 1.55 \mu \mathrm{m}$, GS linewidth of $30 \mathrm{meV}$, and a total Qdot density of $>1.9 \times 10^{13} \mathrm{~cm}^{-2}$ shows the effectiveness of this technique [34]. Later laser device with a 30-stack InAs/InP Qdots active region was also reported [35] and is discussed in section 3.1.1. 
Nevertheless, epitaxial growth of In As Qdots on the (311)B InP substrate suffers from device processing problems like mirror cleaving, anisotropic etching, etc. Therefore, growth of Qdots on conventional (100) InP substrate was required and has been addressed by various research group concurrently which is reviewed below.
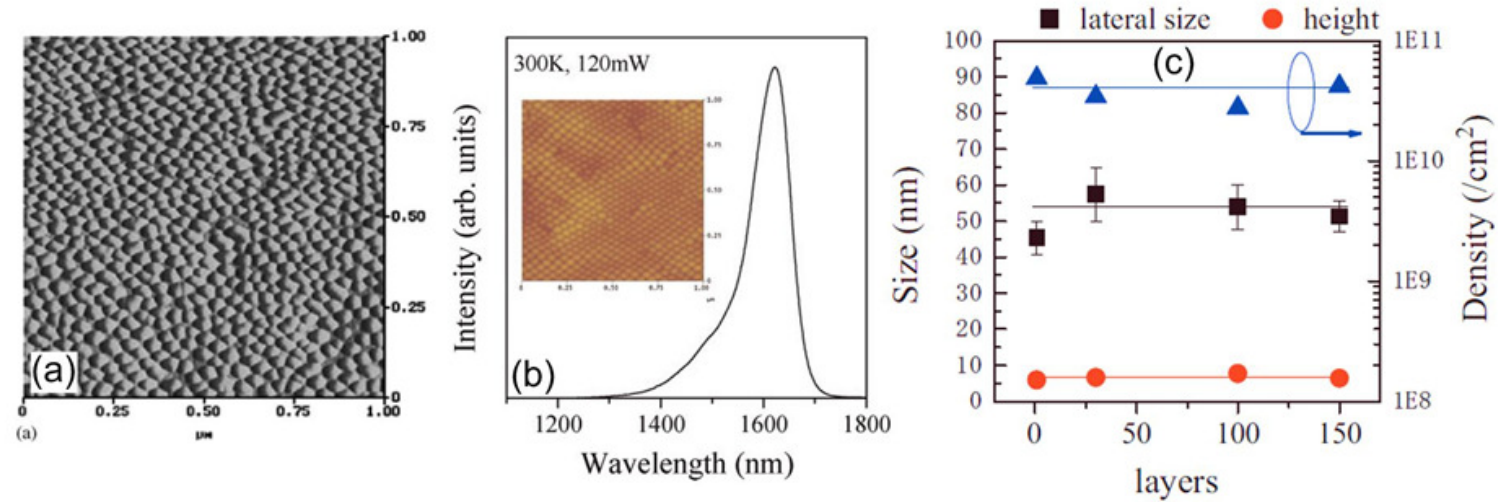

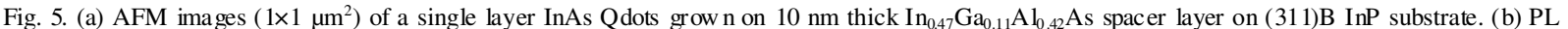
spectrum of a 300 layer stack of InAs Qdots measured at room temperature. (c) Stacking layer dependence of size and density of Qdots. The inset in (b) is an AFM image of the 300th layer of InAs Qdots stack. Courtesy of $[32,34]$.

\subsubsection{Qdots on (100) InP substrate}

MBE growth of InAs Qdashes instead of Qdots on InGaAlAs buffer layers has been explored to elucidate their growth kinetics and provide key insights for eventual realization of InAs Qdots on (100) InP substrate. For instance, the role of buffer layer surface morphology and alloy contents was examined in Ref. [22] as they affect the formation of the nanostructures; growth of high density InAs nanostructures resembling like elongated Qdots [36]; sparsely populated InAs nano-islands [37] on InGaAs, InAlAs, and InP buffer layers on (100) InP [23]. In this respect, Stinz et al. [38] suggested the utilization of vicinal (100) InP substrates for the growth of InAs Qdots. From the MBE growth of In As Qdots on lattice matched InGaAlAs barriers and utilizing vicinal (100) InP substrate, it was found that the Qdot formation was favored when the step edges run perpendicular to the direction of Qdashes i.e. tilted towards (111)B direction while the other direction (111)A shows no change when compared to the exact (100) direction. It was postulated that this shape transition to Qdots occurred because of the local strain field occurring at the steps. The PL emission on capped structures at room temperature was in the wavelength range from 1.5-2.1 $\mu \mathrm{m}$. Similar observation of Qdot formation on (311) B InP substrate by MBE was reported in Ref. [30]. The room temperature PL emission up to $2.1 \mu \mathrm{m}$ and PL linewidth $\sim 70 \mathrm{meV}$ was reported. Furthermore, growth of In As Qdots was also noticed under MOCVD growth on lattice matched In GaAlAs buffer layer on $2^{\circ}$ misoriented (100) InP substrate towards the nearest $<011>$ direction [39]. It was found that when increasing the $\mathrm{Al}$ content in the buffer layer, on top of which Qdots were grown, the PL emission wavelength was blue shifted in conjunction with the growth of smaller Qdot size and higher Qdot density.

Instead of using vicinal InP substrate, Li et al. [40] proposed and assisted growth technique for the MBE growth of InAs Qdots on exact (100) InP. Through the introduction of a lattice-matched thin $(\sim 5 \mathrm{~nm})$ In GaAlAs underlying layer on AlIn As buffer layer, a high quality InAs Qdots with high uniformity has been demonstrated by exploiting the strong dependence of the nanostructure morphology on the characteristics of the underlying alloy. For InAs deposited directly on InAlAs buffer layer, Qdashes elongated along [-110] are formed with an average $\sim 25 \mathrm{~nm}$ width and $3.5 \times 10^{9}$ $\mathrm{cm}^{-2}$ density while employing the assisted growth technique, round Qdots with $\sim 45 \mathrm{~nm}$ in size and $2.5 \times 10^{9} \mathrm{~cm}^{-2}$ in density were observed.

In another study, Kim et al. utilized thin underlying GaAs [36] and In GaAs [41] layer as a means to control the optical and structural properties of InAs Qdots grown on latticed matched In GaAlAs matrix on (100) InP substrate. Increasing the GaAs layer thickness from $0.8 \mathrm{~nm}$ to $1.6 \mathrm{~nm}$ blue shifted the PL emission wavelength and resulted in reduced PL intensity. This was attributed to 
the decrease in the Qdot size and high GaAs potential barrier, and reduction in Qdots density, respectively. In addition, uniform Qdots size is obtained with GaAs underlying layer compared to the Qdots directly grown on InGaAlAs matrix, as reflected by the PL linewidth of $70 \mathrm{meV}$ in the former case and $80 \mathrm{meV}$ in the latter case. Using the $\operatorname{In}_{0.32} \mathrm{Ga}_{0.68} \mathrm{As}$ underlying layer, similarly uniform Qdots were observed with an average lateral size and height of $42 \mathrm{~nm}$ and $3 \mathrm{~nm}$, respectively, and PL linewidth $64 \mathrm{meV}$ centered at $\sim 1.55 \mu \mathrm{m}$. The improvement in Qdots size uniformity was ascribed to the different growth front, growth behavior of group III elements at the interface between Qdots and barrier, and the modulation in the strain field. Surprisingly, growth of nanostructures directly on AlInAs lattice matched matrix layer without any underlying layer show Qdots formation with slight elongation along [1-10] direction, as seen in Fig. 6(a), consistent with the results reported by Brault et al. [23], while the elongation significantly reduced on InAlGaAs matrix layer (see Fig. 6(b)). However, this observation is in contrast to the work reported by Li et al. [40] who reported dash formation on AlInAs buffer layer with similar amount of InAs deposition ( $5 \mathrm{ML})$. Thus, this disparity cannot be attributed to the different amounts of In As deposition as reported by Stintz et al. [38] who observed: (i) Qdash formation at low InAs coverage above the critical thickness, and (ii) transformation into Qdots on increasing InAs coverage. This shows that the resulting nanostructures are highly sensitive to the properties of the surface and the growth conditions. The InAs Qdots on $\mathrm{In}_{0.32} \mathrm{Ga}_{0.68}$ As were observed to be larger in size as depicted in Fig. 6(c).
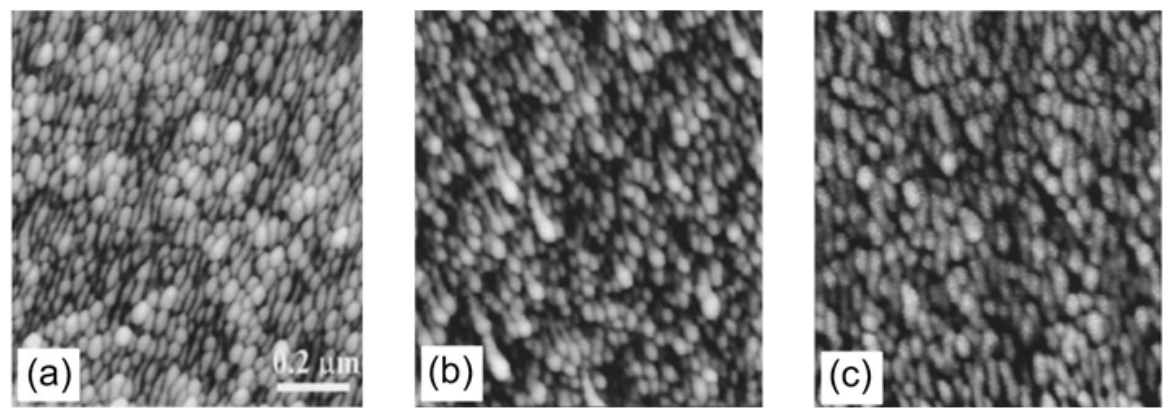

Fig. 6. AFM images $\left(1 \times 1 \mu \mathrm{m}^{2}\right)$ of $5.6 \mathrm{ML}$ InAs Qdots grown on: (a) AlInAs, (b) InA $\mathrm{GaAs}$, and (c) $11.5 \mathrm{~mm}$ thick $\mathrm{In}_{0.32} \mathrm{Ga}_{0.68} \mathrm{As}$ on InAlGaAs. Taken from [41]
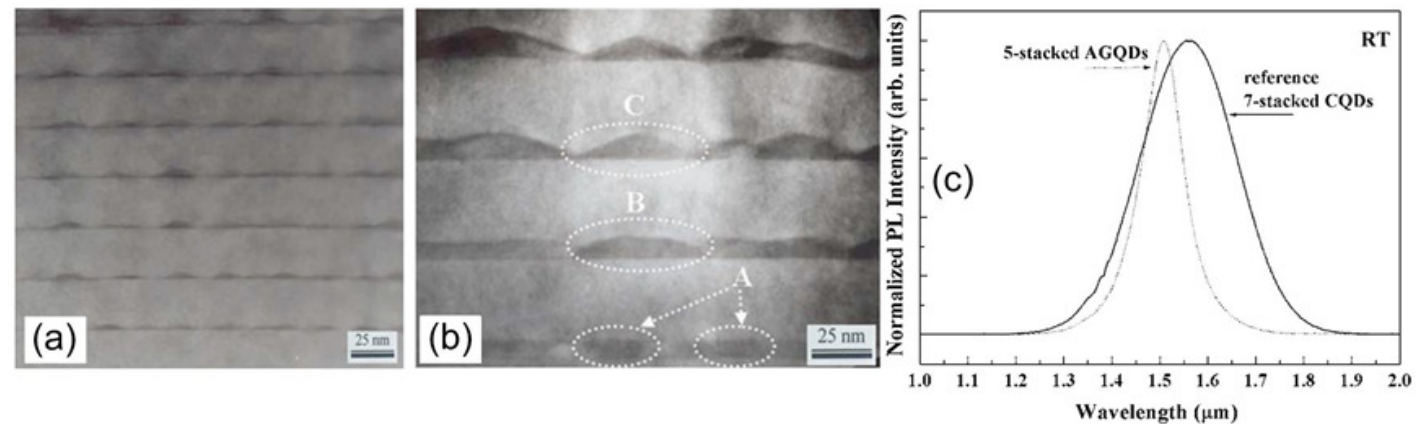

Fig. 7. TEM micrographs of: (a) seven stack CQDs and (b) five stack AGQDs. (c) Room temperature PL spectra of the two samples. Collected from [42].

Instead of changing the buffer layer alloy or composition for the growth of InAs Qdots on (100) InP substrate, Kim et al. [25] explored the alteration in the growth of In As Qdots. They presented an alternate growth method, i.e. AGQD where an InAs layer with a thickness of $1 \mathrm{ML}$ and an InAlGaAs layer with thickness of $1 \mathrm{ML}$ were alternately deposited. Single stack sample was grown by MBE on In GaAlAs buffer layer. The average lateral size and height of AGQDs were 40 $\mathrm{nm}$ and $\sim 10 \mathrm{~nm}$, respectively, and aspect ratio (height/width) $\sim 0.25$; while the corresponding values of the conventionally grown reference InAs Qdots (CQD) were $\sim 26 \mathrm{~nm}$ and $\sim 2.5 \mathrm{~nm}$, and aspect ratio $\sim 0.1$. They attributed the formation of Qdots to the initial accumulated strain arising from the large lattice mismatch between the In As layer and the InAs/GaAs layer. In addition, they also ascribed to the strong influence of locally directional migration of In, Al, and Ga adatoms during the 
deposition of the thin InGaAlAs layer on the Qdots nucleation sites due to the surface chemical potential. The observation of well-defined GS at $1.485 \mu \mathrm{m}$ and excited state (ES) at $1.4 \mu \mathrm{m}$ were reported at room temperature in Ref. [25]. In addition, they also compared a multi-stack grown InAs Qdots based on AGQD (5-stack) and the CQD (7-stack). The corresponding aspect ratio obtained were $\sim 0.22$ and $\sim 0.1$ [42] and is shown in the transmission electron micrograph (TEM) of Fig. 7(a) and (b). The room temperature PL linewidth and emission wavelength from the CQD and AGQDs, depicted in Fig. 7(c), were $85 \mathrm{meV}$ at $1.55 \mu \mathrm{m}$, and $35 \mathrm{meV}$ at $1.5 \mu \mathrm{m}$, respectively, with the latter one among one of the best inhomogeneous broadening value obtained at room temperature on In As/InP Qdots. The optical stability [43] and the effects of group III elements on the growth kinetics of AGQDs were also investigated via utilization of $1 \mathrm{ML}$ InAs and GaAs layers instead of In GaAlAs layer during the growth of InAs Qdots on (100) InP substrate [44].

Recently, in an attempt to realize In As Qdots on (100) InP using MBE, Gilfert et al. [45] found that the arsenic species had a dramatic effect on the formation of nanostructures on In GaAlAs buffer layer. The $\mathrm{As}_{4}$ supply during InAs deposition resulted in Qdash formation on InGaAlAs whereas $\mathrm{As}_{2}$ supply produced round-shaped Qdots structures with density $2.0 \times 10^{10} \mathrm{~cm}^{-2}$, as shown in Figs. 8(a) - (c). The transformation in nanostructure growth was attributed to different incorporation mechanisms of arsenic species. Eliminating the process of cracking $\mathrm{As}_{4}$ into two $\mathrm{As}_{2}$ molecules at the surface by direct supply of As 2 severely lowered the anisotropic migration of indium adatoms along the [0-11] direction due to the stable As-terminated atomic steps along this direction under $\mathrm{As}_{2}$ flux. Under optimized growth conditions of 6 ML InAs deposition and 15 V/III ratio, an increased surface density of $4.0 \times 10^{10} \mathrm{~cm}^{-2}$ and a PL linewidth of $23 \mathrm{meV}$ at $\sim 1.59 \mu \mathrm{m}$ was reported, which is the record low PL linewid th value at 10K. The results are depicted in Fig. 8(d). Similar InAs Qdots formation using $\mathrm{As}_{2}$ species on InAlAs buffer layer on (100) InP was also reported by Fafard et al. [46] more than a decade ago.
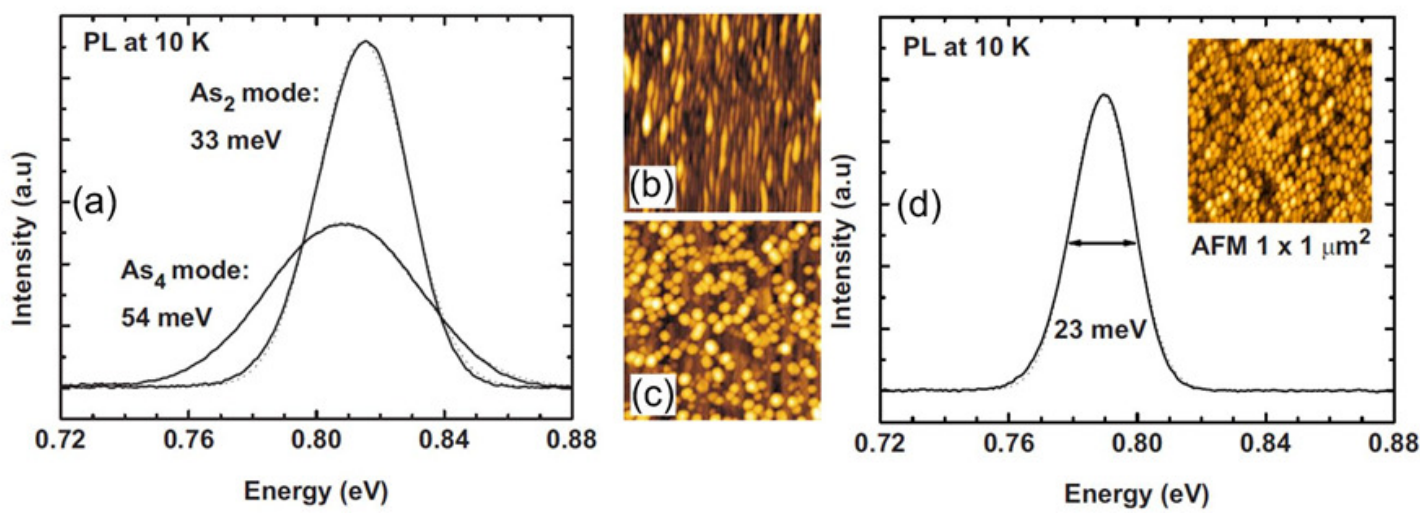

Fig. 8. (a) PL spectra measure d at $10 \mathrm{~K}$ under non-optimized growth conditions and the AFM images $\left(1 \times 1 \mu \mathrm{m}^{2}\right)$ of samples grown using (b) As $\mathrm{s}_{4}$ and (c) $\mathrm{As}_{2}$ fluxes. (d) PL spectrum at $10 \mathrm{~K}$ and AFM image (inset) of the optimized $\mathrm{As}_{2}$ sample with $6 \mathrm{ML}$ InAs and a V/III ratio of 15 . In (a) and (d) the solid lines are experimental data and the dashed lines indicate the Gaussian fits. Taken from [45].

\subsection{InAs/InGaAsP Material System}

This material system has smaller lattice mismatch which resulted in relatively large Qdots. It also suffers from issues related to As/P exchange during InAs growth on InP/InGaAsP buffer layer [47], which inevitably contributes to the Qdots with emission wavelength beyond $1.6 \mu \mathrm{m}$. For instance, Yoon et al. [47] found that the areal density, size distribution, and shape of MOCVD grown InAs self-assembled Qdots chan ged significantly with temperature and V/III ratio with maximum reported density $4.5 \times 10^{10} \mathrm{~cm}^{-2}$. Michon et al. showed that decreasing the trimethyl-indium (TMI) flow rate [48] i.e. InAs growth rate or InP capping layer growth rate [49] decreased the dot density while maintaining PL peak at $1.58 \mu \mathrm{m}$, reaching value as low as $9 \times 10^{7} \mathrm{~cm}^{-2}$. These findings were ascribed to the As/P exchange reaction at the surface which played an important role in the growth kinetics of In As Qdots on (100) InP substrates. In brief, the P atoms on the InP/InGaAsP surface buffer layer are 
easily exchanged by As atoms and deteriorate the interface quality when the surface is exposed to an As-bearing ambient. In the growth of In As Qdots the strain field around the Qdots may make the As/P exchange reaction even more complicated, significantly altering the kinetic processes of the dot formation which are functions of the MOCVD, CBE, and MBE growth conditions. In the next section, we discuss the InAs/InP Qdot growth utilizing these growth techniques.

\subsubsection{Qdots on (100) InP substrate}

Using (100) InP vicinal substrates with $2^{\circ}$ misorientation towards (110), Notzel et al. [50] reported a comprehensive study of different MOCVD growth conditions to obtain InAs/InP Qdots. Increasing the Qdot growth temperature increased the dot size with diameter and height of more than $\sim 100 \mathrm{~nm}$, and $\sim 15 \mathrm{~nm}$, respectively, thus achieving PL emission beyond $1.6 \mu \mathrm{m}$; while at reduced growth temperature, the corresponding Qdot size significantly reduced to $\sim 4 \mathrm{~nm}$ and $\sim 50 \mathrm{~nm}$. This was primarily attributed to the suppression of As/P exchange during low temperature growth which also helped in increasing the Qdots density considerably to around $10^{10} \mathrm{~cm}^{-2}$ [51]. In addition, an ultrathin ( 2 ML) GaAs interlayer sandwiched between the InGaAsP buffer layer and the InAs Qdots layer was also proposed to control the emission wavelength of the Qdots. The average Qdot height reduced from $6 \mathrm{~nm}$ to $3.1 \mathrm{~nm}$, and the peak PL emission wavelength blue shifted from longer than $1.6 \mu \mathrm{m}$ to $1.58 \mu \mathrm{m}$, measured on the sample grown with and without the GaAs interlayer, respectively. This was ascribed to the effective suppression of As/P exchange during InAs Qdots growth by the GaAs layer, and also to the consumption of surface-segregated In on the InGaAsP layer, leading to reduction of the Qdots size. An additional flushing step of trimethyl-gallium (TMG) under tertiarybutyl-arsine (TBA) after the interlayer growth was also employed to ensure the presence of pure TMI as group III source for Qdots growth. This gas switching sequence was done to minimize the composition inhomogeneities in the Qdot layer. Similar reduction in the Qdot size was also observed on reducing the V/III ratio which effectively controls the As/P surface exchange reaction [52] as illustrated in Fig. 9(a). It was also indicated that the thickness of the GaAs interlayers can be used as a parameter to tune the emission wavelength of the InAs Qdots, as shown in Fig. 9(b) and (c). The reduction in the GaAs interlayer thickness from 2 ML to 1.2 ML red shifted the PL emission wavelength of a single stack sample with improved PL efficiency. The resultant Qdots height increased from $4.5 \mathrm{~nm}$ to $5.6 \mathrm{~nm}$, respectively, a direct result of continuous reduction of As/P exchange and consumption of surface-segregated In with GaAs layer thickness. Qdashes elongated along [0-11] were shown to emerge for GaAs layer smaller than 1ML, as depicted in Fig. 9(b), due to low group $\mathrm{V}$ flow, which changes the properties of the InGaAsP surface to cause anisotropic surface diffusion along [0-11] leading to Qdash formation for sub-monolayer GaAs coverage. In fact, the GaAs interlayer assisted growth method was also shown to successfully reproduce identical Qdots stacks with thick separation layers, as summarized in Fig 9(c) [53]. With increasing InAs deposition and GaAs layer thickness, the tuning of PL emission wavelength of the multi-stacked InAs/In GaAsP Qdots was possible.
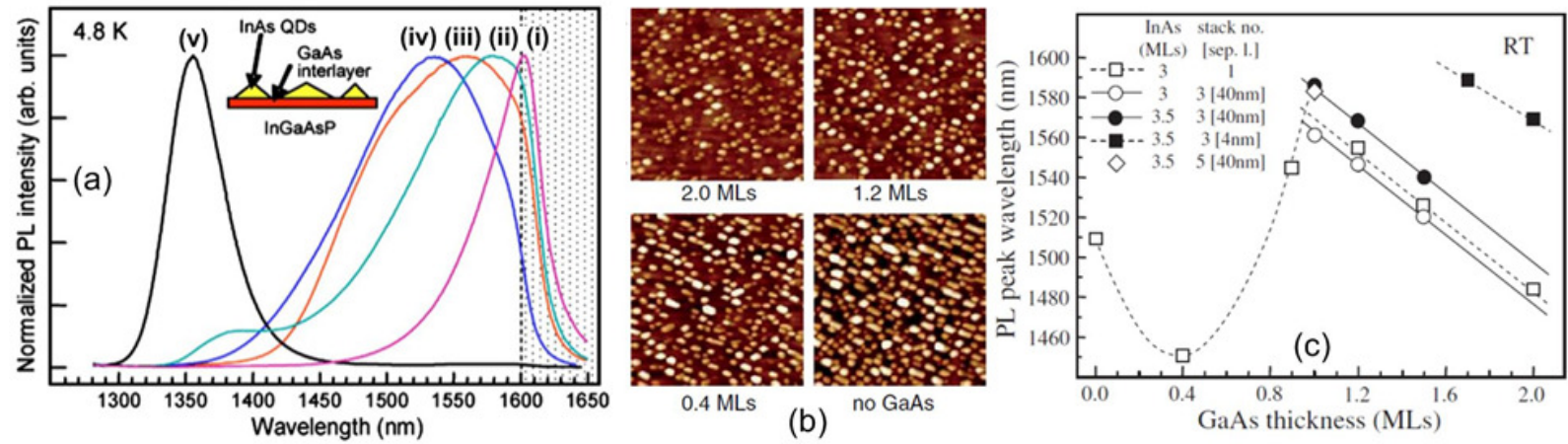

Fig. 9. (a) N ormalized PL spectra at $4.8 \mathrm{~K}$ of the 3 ML InAs single Q dot layer grown: (i) without, and (ii) with 2 ML GaAs interlayer. (iii)-(v) PL spectra of Qdots with $2 \mathrm{ML} \mathrm{GaAs}$ interlayer and $45 \mathrm{~s}$ flushing time grown at TBA flow rates of (iii) 6.1, (iv) 2.0, and (v) $1.0 \mathrm{sccm}$. The shaded area in (a) is above the detection limit at $1.6 \mu \mathrm{m}$. (b) AFM images $\left(1 \times 1 \mu \mathrm{m}^{2}\right)$ of $3 \mathrm{ML}$ InAs single stack Qdots with different GaAs interlayer thickness. (c) PL 
emission wavelength at room temperature as a function of GaAs interlayer thickness of single and vertically stacked InAs Qdot layers formed using various amount of InAs, InGaAsP separation layer thicknesses, and number of Qdots stacks. The inset of (a) shows a single InAs Qdots structure with GaAs interlayer on InGaAsP buffer layer. Reproduced from [50, 52, 54].

Rather than tuning the emission wavelength of Qdots via changing the buffer layer surface growth kinetics, Jang et al. [55] utilized Qdots material composition instead. In(Ga) As/In GaAsP Qdots were grown on exact (100) InP substrate using MOCVD technique. By controlling the composition of $\operatorname{In}(\mathrm{Ga})$ As Qdots material, PL emission in the range of 1.35 to $1.65 \mu \mathrm{m}$ was achieved. The Qdots size increased while the height decreased with the increase in the relative concentration of TMG to TMI during the Qdot growth. In addition, Qdot density as high as $1.13 \times 10^{11} \mathrm{~cm}^{-2}$ was obtained with higher concentration of group III alkyl supply. A 5-stack $\operatorname{In}(\mathrm{Ga})$ As/InGaAsP Qdot sample showed a PL emission and linewidth of $1.5 \mu \mathrm{m}$ and $63 \mathrm{meV}$, respectively, at room temperature [56]. In another study, Michon et al. [57] showed that additional $P$ flux during the MOCVD growth of InAs Qdots on InP buffer layer also allowed tuning of Qdots emission around $1.55 \mu \mathrm{m}$. It was shown that increasing the $\mathrm{PH}_{3} / \mathrm{AsH}_{3}$ ratio from 0 to 50 blue shifted the PL peak wavelength from $\sim 1.88 \mu \mathrm{m}$ to $\sim 1.38 \mu \mathrm{m}$ with a reduced PL linewidth, enlightened by the kinetic effects due to additional $\mathrm{P}$ flow and energetic effects due to the reduction in the lattice mismatch between Qdots material and (100) InP substrate.

Growth of In As Qdots on exact (100) InP by means of CBE was introduced by Poole et al. [58], via depositing a thin 2-5 ML of InAs on a buffer layer followed by a growth interruption under As overpressure to avoid surface degradation, similar to the work reported by Fafard et al. on MOCVD grown InAs/InP Qdots [24]. A clear transformation from a two dimensional (2D) Qwell state to one dimensional Qdash state elongated along [0-11] direction, and eventually to OD islands was observed as a function of growth interrupt time. The Qdashes were 20-25 nm wide and 40-130 $\mathrm{nm}$ long while the Qdots were 25-40 nm wide. A red shift in the PL emission with decreased PL linewidth indicated a continual increase in Qdot height and uniformity with growth in terruption time. Moreover, a high overpressure of As flux during growth interruption favored Qdot growth while a very low As overpressure resulted in the formation of 2D InAs Qwell layer [58]. Alteration to the growth rate of the Qdot capping layer was also examined via PL measurements, shown in Fig. 10(a), and found that higher growth rate reduced the As/P exchange at the Qdots surface and preserve the Qdot size and shape (indicative of red shift in the PL emission), thus able to control the Qdot emission wavelength. This was required since the buried dots tend to become shorter compared to the surface dots due to exposure to P flux during capping [59] which causes the InP adatoms (formed at the top layer of In As) to migrate towards the region in between the Qdots due to the strain mismatch. This process was further improved by a double capping procedure [60], in troduced by Ledentsov et al. [61] and Gutierrez et al. [62] on InAs/GaAs and InAs/InP Qdots, respectively, as shown in Fig. 10(b). In this modified approach the region between the Qdots was first partially filled with a thin InP capping layer followed by a growth interruption under $\mathrm{P}$ overpressure where the As/P exchange was exploited to trim the taller Qdots which are not buried under th in capping layer compared to shorter Qdots. Later, a thick second capping layer was deposited. This significantly helped in controlling the Qdots height uniformity, and their emission energies.

Different ultra-thin interlayers were also utilized to tune the emission wavelength of InAs/InP Qdots grown by CBE system [63] on (100) InP substrate miscut $2^{\circ}$ towards (110), similar to the MOCVD grown InAs/InP Qdots discussed above. As depicted in Fig. 10(c), both GaAs [64] and GaP interlayers blue shifted the emission wavelength due to suppressed As/P exchange and consumption of surface segregated In. The PL linewid ths with increasing interlayer thickness remained within 45$55 \mathrm{meV}$ at $4.8 \mathrm{~K}$ and the PL efficiencies were comparable. Smaller coverage of GaP was required to obtain the same wavelength blue shift compared to the GaAs coverage which was attributed to better effectiveness of $\mathrm{GaP}$ in suppressing the As/P exchange, as the $\mathrm{GaP}$ bond strength is stronger in the InGaAsP material system compared to GaAs [65].

High density InAs/InGaAsP Qdots active region are highly desirable in realizing optoelectronic devices for photonic integrated circuits. This motivated the research community to continue growing Qdots on (311)B InP substrates [66] instead of (100) InP substrate for device demonstrations. Balancing the built-in strain caused by Qdots became crucial in a multi-stack 
structure, since the generated strain field in the subsequent capping layers causes variation in its composition leading to preferential Qdot growth from layer to layer. This in turn encourages the Qdot size to grow resulting in degradation of Qdot uniformity, as observed in the InAs/GaAs material system [67]. Combining the double capping procedure and employing a GaP interlayer, Poole et al. [68] was able to stack 1 to 10 layers of In As/InGaAsP Qdots without any change in PL peak wavelength or PL linewidth, as shown in Fig. 10(d). Furthermore, tuning the emission wavelengths of the Qdots was also demonstrated by means of InAs deposition rate and the first capping layer thickness. A red shift in the PL peak wavelength was observed on increasing the thickness of first cap or decreasing the In As deposition rate which was ascribed to the change in the Qdots height as a consequence of As/P exchange process [68].
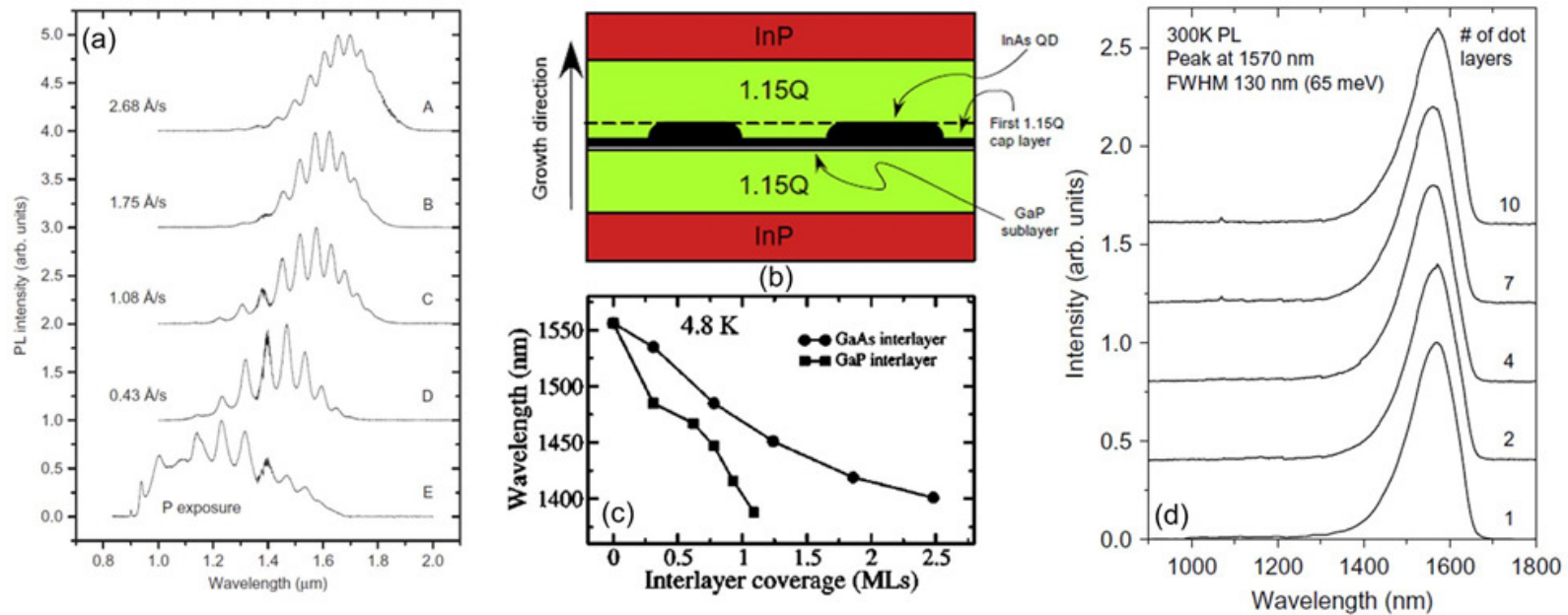

Fig. 10. (a) Normalized PL spectra of InAs/InP Qdots as a function of growth rate of InP capping layer at $4 \mathrm{~K}$. (b) A single double capped layer structure with underneath GaP layer. (c) Tuning capability obtained from the PL emission wavelength at $4.8 \mathrm{~K}$, of a single InAs/InP Qdot layer on a $\mathrm{GaP}$ and GaAs interlayer, as a function of intelayer coverages. (d) Normalized PL spectra of InAs/InP Qdots as a function of number of stacking layers at $300 \mathrm{~K}$, and utilizing double capping technique with $\mathrm{GaP}$ underlayer. Adapted from $[65,69,70]$.

MBE growth of InAs/In GaAsP Qdots was carried out by Li et al. [71] and Elias et al. [72] on exact and vicinal (100) InP substrates, respectively. Without assistance of any additional growth techniques which is normally required in MOCVD and CBE growth of InAs/InGaAsP Qdots, a $10^{10}$ $\mathrm{cm}^{-2}$ areal density of Qdot is reported with room temperature PL emission wavelength and linewidth of $1.55 \mu \mathrm{m}$ and $108 \mathrm{meV}$, respectively. The Qdots were slightly elongated along the [01-1] direction with mean diameter and height of $76 \mathrm{~nm}$ and $2.9 \mathrm{~nm}$, respectively, similar to the InAs/In AlGaAs Qdots grown in MBE by Kim et al. [41], discussed in section 2.1.2. By adjusting the thickness of the In As layer and the growth temperature, wavelength tuning of the Qdots was achieved [73]. It was observed that increasing the InAs layer thickness beyond $3 \mathrm{ML}$ transforms the Qdot-like structures to Qdashes elongated along [0-11] direction with red shifting of the PL emission wavelength. This was attributed to the In migration on the surface of InGaAsP along [100] direction. A value of 3.5 ML was suggested to be the critical thickness for the formation of Qdots.

Recently, Jo et al. [74] explored utilization of Qdot-in-a-well (DoWELL) structure to improve the laser device performance. In As Qdots were sandwiched between $1.35 \mu \mathrm{m}$ InGaAsP Qwell and further embedded in $1.15 \mu \mathrm{m}$ barrier layer on (100) InP substrate. GaAs interlayer was utilized to maintain the Qdot wavelength tuning for a seven stack structure. The Qdot density in DoWELL structures were found to be slightly lower $\left(\sim 2 \times 10^{10} \mathrm{~cm}^{-2}\right)$ than that of Qdots on InGaAsP buffer layer without GaAs interlayer $\left(\sim 2.6 \times 10^{10} \mathrm{~cm}^{-2}\right)$. This was believed to be due to alteration in the migration characteristics of In atoms on GaAs surface during the In and As precursor supply. The PL linewidth and efficiency of DoWELL structures substantially increased (from $92 \mathrm{meV}$ to 68 $\mathrm{meV}$ ) and was attributed to a combined effect of enhanced carrier capturing [74]. This is a wellestablished technique utilized in InAs Qdots on GaAs substrate system for device performance improvement [2]. 
The thrust to increase the InAs Qdot density on (100) InP substrate rather than relying on (311)B InP substrate which is incompatible with the standard laser fabrication process, led to the exploration of misoriented substrates. These substrates helped in modifying the In diffusion length arising from the atomic steps thus resulting in more isotropic nanostructure formation, as discussed initially in this section. Elias et al. [72] employed a growth interruption step after InAs deposition on $1.18 \mu \mathrm{m} \mathrm{InGaAsP}$ buffer layer for $30 \mathrm{~s}$ under As overpressure, and compared the nanostructure formation on the nominal $(\mathrm{N})$, and $2^{\circ}$ off-cut towards (111)A and (111)B, (100) InP substrates forming surface steps namely; A steps and B steps which will be used henceforth. At high As flow rate, larger $\left(\sim 50 \mathrm{~nm}\right.$ diameter) and lower density $\left(\sim 2 \times 10^{10} \mathrm{~cm}^{-2}\right)$ Qdots were formed independent of the substrate orientation. However, at low As flow rate, smaller and higher density Qdots were formed on vicinal (100) InP miscut towards (111)B while the other two substrates showed Qdash formation, as shown in Fig. 11. This was attributed to the lower step energy of In As A steps (along [1-10]) than B steps (along [110]) and thus of the shorter diffusion length of In in the [110] direction. Furthermore, the effect of growth temperature did not significantly change the Qdot structural and optical characteristics which indicated that the effective diffusion length of the adatoms on the surface is limited by the presence of steps. An improvement in the Qdot density from $7 \times 10^{10} \mathrm{~cm}^{-2}$ to $9 \times 10^{10} \mathrm{~cm}^{-2}$ and a weak decrease in average dot height (from 2 to $1 \mathrm{~nm}$ ) and diameter (from $35 \mathrm{~nm}$ to $26 \mathrm{~nm}$ ) were observed on decreasing the growth temperature [75]. In brief, an areal InAs/InGaAsP Qdot density on exact (100) InP substrate was measured in the range of $\sim 2 \times 10^{10}-4 \times 10^{10} \mathrm{~cm}^{-2}$ and up to $9 \times 10^{10} \mathrm{~cm}^{-2}$ from vicinal substrates [72].

(a)
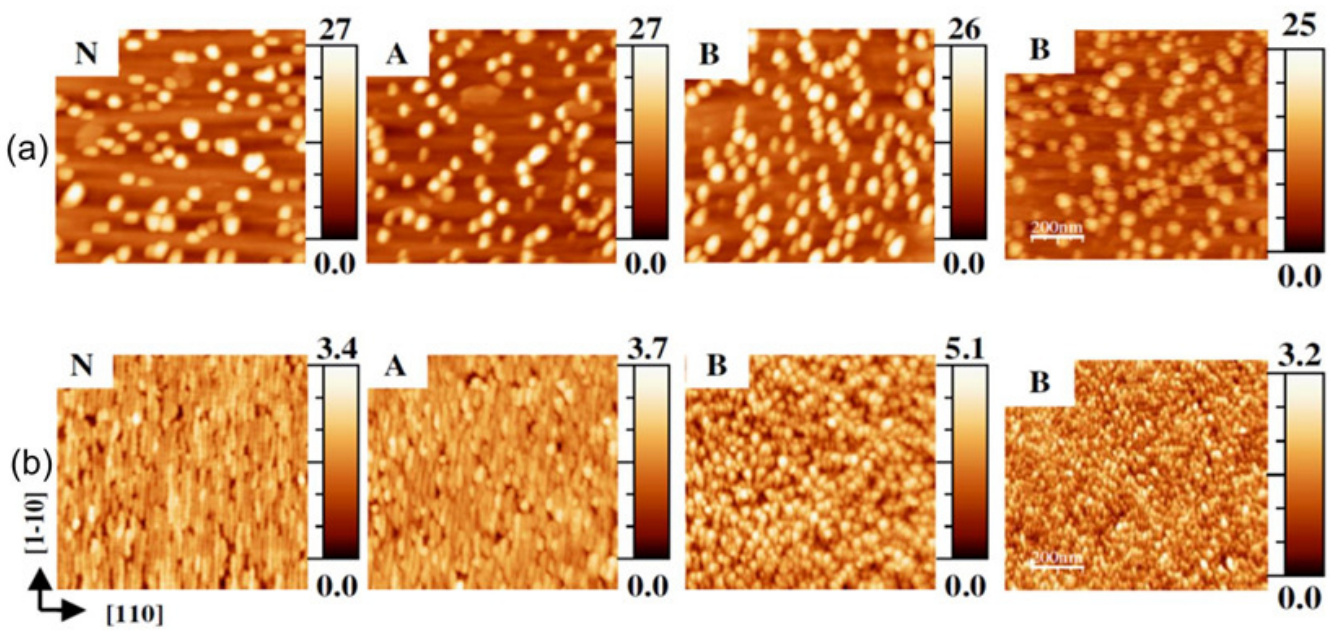

Fig. 11. AFM images $\left(1 \times 1 \mu \mathrm{m}^{2}\right)$ of InAs/InP nanostructures deposited on nominal N, A, and B surfaces under two different As fluxes at (a) 6 and (b) $0.3 \mathrm{sccm}$. The first three columns from the left were grown at $480^{\circ} \mathrm{C}$ while the last column was grown at $400{ }^{\circ} \mathrm{C}$. The height $\mathrm{contrast} \mathrm{scale}$ is in nm. Courtesy of [75].

\subsubsection{Qdots on (311)B InP substrate}

Growth of InAs Qdots on the high index (311)B InP substrate permitted the reproducible formation of dense and small size Qdots when compared to Qdots grown on the nominal (100) InP substrate, under same growth conditions. This was demonstrated by Frechengues et al. [59] on InAs/InGaAsP material system grown by MBE. A dot density of $5 \times 10^{9} \mathrm{~cm}^{-2}$ was reported on (100) InP with highly inhomogeneous large size Qdots (70 nm diameter and $16 \mathrm{~nm}$ height) whereas $5 \times 10^{10} \mathrm{~cm}^{-2}$ density was obtained on the (311)B InP with uniform small dots (35 nm diameter, and $8 \mathrm{~nm}$ height), as shown in Fig.12 (a) and (b). Furthermore, tunability of the Qdot emission wavelength was obtained by annealing the InAs Qdots under P overpressure before the InGaAsP capping layer growth, and the substrate temperature. By this modification of the As/P flux sequence a wavelength tunability of $>$ $250 \mathrm{meV}$ was obtained at high substrate temperature around $1.55 \mu \mathrm{m}$ and is illustrated in Fig. 12(c).

Dispersive size Qdots are highly undesirable as they deteriorate a laser performance if used in active region. This issue was addressed by Paranthoen et al. [76] on (311)B InP substrate by 
demonstrating reduced PL linewidth via double capping technique, discussed previously [60], on the MBE grown InAs/InGaAsP Qdots also. It was shown that the two step capping separated by a growth interruption under $\mathrm{P}$ flux decreased the inhomogeneity of Qdots with density and mean dot diameter (height) of $5 \times 10^{10} \mathrm{~cm}^{-2}$ and $30(8) \mathrm{nm}$, respectively. The room temperature PL linewidth drastically reduced from $120 \mathrm{meV}$ to $50 \mathrm{meV}$ at $\sim 1.55 \mu \mathrm{m}$ using this modified technique. Moreover, a similar red shift in the PL emission wavelength was observed on increasing the thickness of the first InP cap, as seen in the CBE grown InAs/InGaAsP Qdots, and was attributed to the As/P exchange procedure [77]. In continuation to this work, Caroff et al. looked into the effect the first In GaAsP capping layer thickness (see Fig. 13(a)), different growth interrupts overpressure types viz. As, $\mathrm{P}$ and As and $\mathrm{P}$, and the growth interrupt duration, on the Qdots emission [78]. A red shift (blue shift) in PL peak wavelength was observed on decreasing (increasing) the first cap thickness under As ( $\mathrm{P}$ or As and $\mathrm{P}$ ) which was discussed in terms of the compressive (tensile) stress experienced by In GaAsP first capping layer due to InAs (P-rich) surface as a result of alteration in the As/P exchange process. Qdots emission tuning capability from $\sim 1.3 \mu \mathrm{m}$ to $\sim 1.8 \mu \mathrm{m}$ was possible. In another study Ulloa et al. [79] showed that the height of the Qdots correspond to the thickness of the first cap layer up to $3.5 \mathrm{~nm}$ which indicated the double capping procedure's effectiveness in controlling the Qdots height.
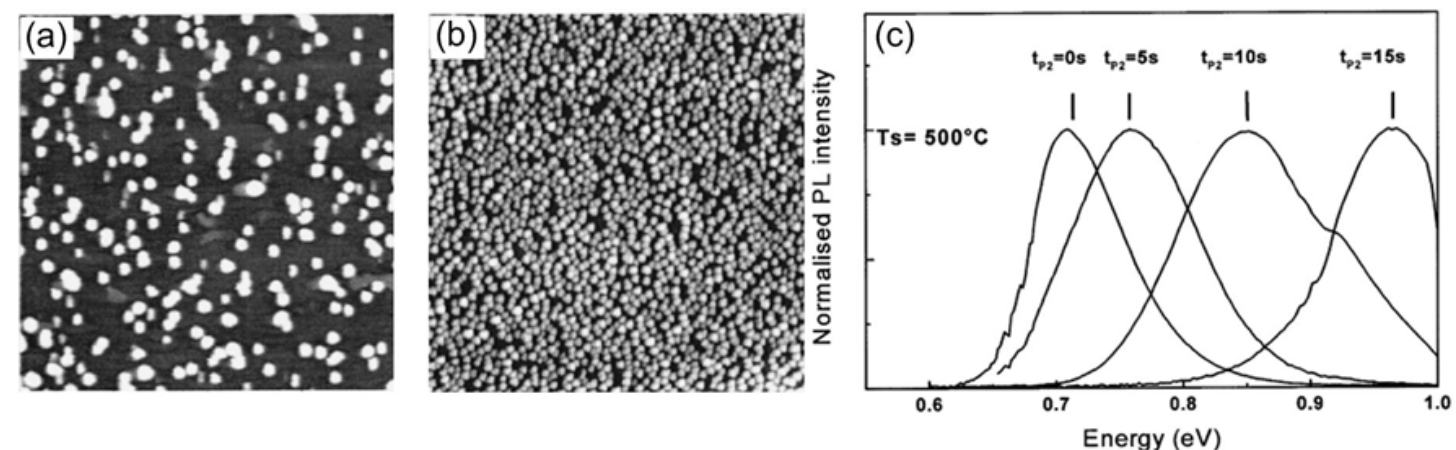

Fig. 12. AFM images $\left(2 \times 2 \mu \mathrm{m}^{2}\right)$ of the uncapped InAs Qdots on (a) (100) InP and (b) (311)B InP substrate. (c) PL spectra of the InAs Qdots on (311)B InP substrate after a growth interruption under $\mathrm{P}$ overpressure for various times at the substrate temperature of $500^{\circ} \mathrm{C}$. Taken from [80].
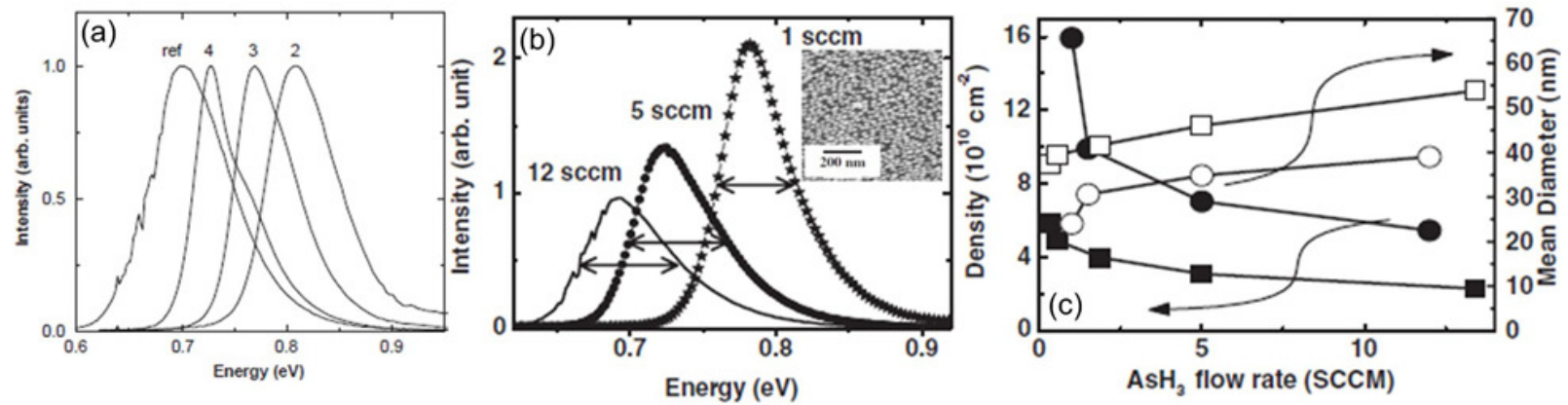

Fig. 13. R oom temperature PL spectra of (a) double capped samples with first InGaAsP (Q1.18) cap layer thicknesses of 4, 3 and $2 \mathrm{~nm}$ and a growth interrupt under a combined $\mathrm{As}_{2}$ and $\mathrm{P}_{2}$ overpressure during $120 \mathrm{~s}$. (b) Single capped InAs/InGaAsP Qdot samples with different As flow rates during the growth interruption of $30 \mathrm{~s}$ after InAs deposition. (c) Changes in Qdots density (filled symbols) and mean diameter (empty symbols) versus As flow rate during the growth interruption of $30 \mathrm{~s}$ after InAs deposition on InGaAs (square symbols) and InGaAsP (circle symbols) buffer layers. The inset of (b) is an AFM images of the unc apped InA s/InGaAsP Qdots at an As flow rate of $1.0 \mathrm{sccm}$. Taken from $[31,78]$.

With already noteworthy accomplishment in In As/InP Qdots density reaching $\sim 5.5 \times 10^{10} \mathrm{~cm}^{-2}$ on (311)B InP substrate, Caroff et al. [31] further explored the possibility for attaining values beyond $10^{11} \mathrm{~cm}^{-2}$ as this value was already achieved in InAs/InAlGaAs Qdots system on (311)B substrate (see section 2.1.1). By reducing the As flux during In As Qdots formation, it was shown that the Qdot density dramatically increased up to $1.6 \times 10^{11} \mathrm{~cm}^{-2}$ and the room temperature PL linewidth decreased from $70 \mathrm{meV}$ at $12 \mathrm{sccm}$ As flux to $50 \mathrm{meV}$ at $1.0 \mathrm{sccm}$, as depicted in Fig. 13(b) [81]. Furthermore, the Qdots height (diameter) decreased from 7.7 (19.5) $\mathrm{nm}$ to 4.8 (12) $\mathrm{nm}$, thus, blue shifting the PL peak wavelength from $\sim 1.79 \mu \mathrm{m}$ to $\sim 1.58 \mu \mathrm{m}$. This trend of the effect of As overpressure was found 
to be independent of the buffer layer nature as shown in Fig. 13 (c), and hence was related to increase in the strained In As (311) B surface energy on decreasing As overpressure which in turn increased the instability of the growth front and favored Qdots nucleation leading to large Qdots density.

Stacking of InAs/InGaAsP Qdots on (311)B InP utilizing double cap technique was also proven by Bertru et al. as highly efficient in maintaining the Qdot uniformity with a spacer layer thickness around $40 \mathrm{~nm}$ [82]. The room temperature PL emission at $1.55 \mu \mathrm{m}$ with linewidth of 62 meV were maintained for a single stack and six stacks of Qdots, attributed to the reduced mean strain accumulated which drastically reducing the PL energy shift.

\subsection{Post-growth tuning of InAs/InP Qdots}

Instead of achieving tunability of self-assembled Qdot dimensions and composition in order to control the emission wavelength and uniformity during the growth process, post growth bandgap engineering was also carried out to blue shift the InAs/InP Qdots emission towards $1.55 \mu \mathrm{m}$ regime. Different tuning process such as rapid thermal annealing (RTA), impurity-free vacancy disordering (IFVD), laser-induced intermixing (LII), grown-in-defects (GID) mitigated intermixing, and ionimplantation-induced disordering (IID) have been proposed. Girard et al. [83] performed a simple RTA on InAs/(100)-InP Qdots grown by CBE method, and reported a blue shift of up to $120 \mathrm{meV}$ (reference as grown sample PL emission at $\sim 1.55 \mu \mathrm{m}$ ) at $650-800^{\circ} \mathrm{C}$ for $210 \mathrm{~s}$ on both conventional single capped sample and samples with double capping procedure. Chia et al. [84] investigated the post-growth group-V intermixing of MOCVD growth In As/(100)-InP Qdots via IFVD with $\mathrm{SiO}_{2}$ and $\mathrm{Si}_{\mathrm{x}} \mathrm{N}_{\mathrm{y}}$ capping and LII with $1.064 \mu \mathrm{m} \mathrm{CW}$ laser source. A substantial improvement in the PL peak intensity and linewidth was observed with energy blue shift as large as $350 \mathrm{meV}$ from the reference sample emission at $1.65 \mu \mathrm{m}$, as shown in Fig. 14(a). A maximum differential energy shift between the two capped samples at $850^{\circ} \mathrm{C}$ was $90 \mathrm{meV}$. From LII process a differential energy shift of $>250$ meV was demonstrated. In another study Wang et al. [85] reported post growth bandgap tuning of In As/InP Qdots in InGaAlAs Qwell laser structure via $\mathrm{SiO}_{2}$ and $\mathrm{Si}_{\mathrm{x}} \mathrm{N}_{\mathrm{y}}$ capping. The IFVD process resulted in larger bandgap blue shift from the latter cap compared to former one with a differential shift of $92 \mathrm{~nm}$ after annealing at $800^{\circ} \mathrm{C}$ for $30 \mathrm{~s}$. This was related to the dominant In diffusion with respect to other group-III atoms.
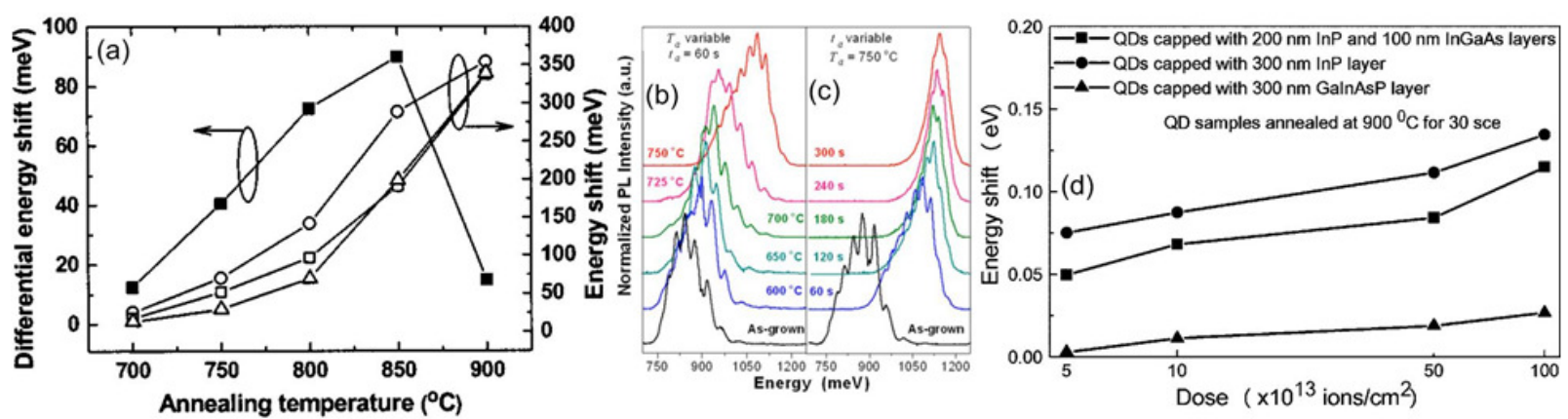

Fig. 14. (a) Energy shift as a function of annealing temperature for samples capped with $\mathrm{SiO}_{2}(\circ), \mathrm{SiN}_{\mathrm{x}}(\Delta)$ and without dielectric cap ( $\square$ ). Also shown is the differential energy shift (-) for samples capped with $\mathrm{SiO}_{2}$ and $\mathrm{SiN}_{\mathrm{x}}$. PL spectra from as-grown and annealed GID samples (b) as a function of annealing temperature $T_{a}$ for an annealing time $t_{a}=60 \mathrm{~s}$ and (c) as a function of $t_{a}$ for $T_{a}=750{ }^{\circ} \mathrm{C}$. (d) The implantation-induced energy shifts of the quantum dots capped with $\mathrm{InP}, \mathrm{InP}$ and $\mathrm{InGaAs}$, and InGaAsP layers and annealed at $900{ }^{\circ} \mathrm{C}$ for $30 \mathrm{~s}$. The implantation dose is varied from $5 \times 10^{13}$ to $1 \times 10^{15}$ ions $/ \mathrm{cm}^{2}$. Collected from $[84,86,87]$.

The GID induced intermixing by introduction of point defects into the epitaxial layers during growth at reduced temperatures was demonstrated by Dion et al. [86]. Energy shifts of $270 \mathrm{meV}$ $(\sim 1.5 \mu \mathrm{m}$ to $\sim 1.1 \mu \mathrm{m})$ from the CBE grown In As/(100)-InP Qdots sample with low temperature grown InP (LT-InP) layer was reported after annealing at $750^{\circ} \mathrm{C}$ for $300 \mathrm{~s}$ and is illustrated in Fig. 14(b) and (c). This was attributed to the presence of a non-equilibrium concentration of point defects in the LT-InP which was confirmed by the progressive etching of this layer which led to the gradual 
quenching of the PL shift. The effect of the influence of post-growth phosphorus implantation [88, 89] and proton-implantation [87] followed by RTA was carried out on the CBE and MOCVD grown In As/(100)-InP Qdots with InP capping layer and with thin GaAs interlayer capped with InGaAs or InGaAsP capping layer. A large P implantation induced blue shift of up to $325 \mathrm{meV}(\sim 1.48 \mu \mathrm{m}$ reference sample) was observed by Dion et al. [88]. On the other hand, Barik et al. [87] reported high proton implantation-induced energy blue shift due to strong group V interdiffusion, and the least blue shift from the Qdots capped with InGaAsP layer due to weak group V and group III interdiffusion. In addition, higher dose decreased the PL linewidth of the samples due to homogenization of Qdots as a result of interdiffusion which is shown in Fig. 14(d).

\subsection{Towards $>2.0 \mu \mathrm{m}$ wavelength emission}

From the discussion in sections 2.1 and 2.2 it is clear that most of reported work was interested in blue shifting the InAs/InP Qdots luminescence emission at $1.55 \mu \mathrm{m}$ in the $\mathrm{C}$-band telecommunication window for optical communications because of their inherent long wavelength emission beyond $1.55 \mu \mathrm{m}$ due to the reduced lattice mismatch between InAs and InP. Attempts to move the In As/InP Qdots emission wavelength other way around and extending beyond $\sim 1.8 \mu \mathrm{m}$ has recently attracted attention owing to the various cross-disciplinary field applications in spectroscopy and sensing [90] as discussed in section 1. In this respect, alteration of various growth parameters that result in the red shifting of InAs/InP Qdots emission; for instance, increasing the thickness of the In As deposition or the growth interruption time before capping the dots or the thickness of the first capping layer in the double capping procedure, etc. could be exploited to attain the desired wavelength. However, the maximum room temperature PL emission wavelength reported from this nanostructure is about $\sim 1.8 \mu \mathrm{m}$. To further increase the emission wavelength, Zhukov et al. [91] proposed and demonstrated utilization of InGaAs matrix layer on (100) InP substrate for the growth of InAs Qdots. $77 \mathrm{~K}$ PL studies as a function of InAs ML deposition on $\mathrm{In}_{0.53} \mathrm{Ga}_{0.47} \mathrm{As} / \mathrm{InP}$ showed a wavelength tunability from $\sim 1.7 \mu \mathrm{m}$ to a maximum of $\sim 1.95 \mu \mathrm{m}$. Another alternative to extend the emission wavelength was to incorporate Sb during the growth of InAs Qdots on InP substrate [92, 93]. Qui et al. [93] reported this possibility by growing InAsSb Qdots on (100) InP substrate using MOCVD. A dot density as high as $4 \times 10^{10} \mathrm{~cm}^{-2}$ was obtained via self-assembled growth by alternating group III and group V precursors, and almost pure InSb dots were formed on $\mathrm{In}_{0.53} \mathrm{Ga}_{0.47} \mathrm{As} / \mathrm{InP}$ substrate with room temperature PL emission at 1.7-2.2 $\mu \mathrm{m}$, as depicted in Fig. 15(a). In another study, Dore et al. [94] also reported a room temperature PL emission at $2.35 \mu \mathrm{m}$ from InAs(Sb) Qdots embedded in InGaAs alloy lattice matched to (100) InP. In this case, after the deposition of InAs, a growth interrupt under $\mathrm{Sb}$ flux was carried out before capping with the InGaAs layer. The result is plotted in Fig. 15(b).
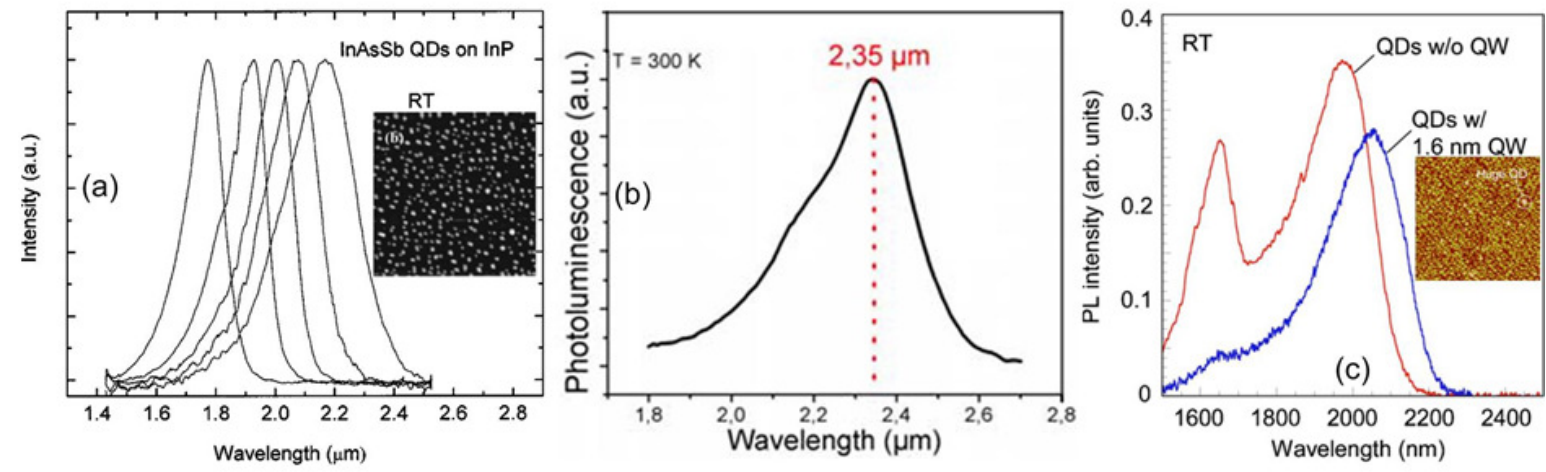

Fig. 15. (a) Normalized room temperature PL spectra of $\operatorname{InAs}(\mathrm{Sb})$ Qdots and almost pure InSb Qdots (emission at $2.2 \mu \mathrm{m})$ self-assembled on InGaAs/(100) InP. (b) R oom temperature PL spectrum of self-assembled InAsS b nanostructures on InGaAs lattice-matched to (100) InP. (c) Room temperature PL spectra of InAs Qdots directly grown on $20 \mathrm{~nm}$ InGaAs and on $1.6 \mathrm{~nm}$ InAs QW. The inset of (a) and (c) are the AFM ima ges of $1 \times 1$ $\mu^{2}$ and $2 \times 2 \mu \mathrm{m}^{2}$ size InAsSb Qdots self-assembled on InGaAs/(100) InP and the extremely dense InAs Q dots on Qwell with a huge QD indicated, respectively. Reproduced from [93-95]. 
Recently, Kotani et al. [95] reported an emission wavelength of $2.06 \mu \mathrm{m}$ from optimized growth conditions of InAs Qdots by capping with $5 \mathrm{~nm}$ lattice matched InGaAs layers before the thick In GaAsP buffer layer deposition on vicinal (100) InP substrate using MOCVD growth method. The red shift in the emission wavelength due to the InGaAs layer was attributed to a larger In adatom surface migration length on InGaAs. In addition, by compressively straining InGaAs layer, a red shift in the PL emission was observed reaching $2.16 \mu \mathrm{m}$. Successively, growth of InAs Qdots on top of $1.6 \mathrm{~nm}$ InAs Qwell (dot-on-well) was also proposed and demonstrated which resulted in an increase of dot density and with room temperature PL emission at $2.05 \mu \mathrm{m}$, as shown in Fig. 15(c). All these efforts shows the potential of InAs/InP Qdots material system in achieving emission beyond $2 \mu \mathrm{m}$, and thus open up routes for further extending the emission wavelength via InAs( $\mathrm{Sb}) / \mathrm{InP}$ Qdots material system with already demonstrated $\mathrm{CW}$ semiconductor laser diode operation from this material system with lasing near $2 \mu \mathrm{m}[96,97]$. 


\section{InAs/InP Qdots Devices}

In this section, the achievements of InAs/InP Qdots as the gain region in various semiconductor devices is reviewed. The section begins with the discussion on the accomplishment of narrow band Fabry-Perot (FP) edge-emitting semiconductor lasers performance. These lasers are based on optimized InAs/InP Qdots active region discussed in section 2. Later, the inherent inhomogeneous broadening from multi-stack InAs/InP Qdots active region which usually results in a broadband gain profile and hence electroluminescence, was exploited to realize broadband semiconductor components such as SOAs and SLDs. In addition, this characteristic also results in broadening of multimode lasing spectra under stimulated emission with increasing current injection due to many Qdots taking part in capturing the carriers. This formed the basis of realizing semiconductor broadband lasers, mode-locked lasers, multi-wavelength and tunable lasers, which are summarized later in this section.

\subsection{InAs/InP Qdots lasers}

The first InA/InP Qdots laser was reported at $77 \mathrm{~K}$ by Ustinov et al. $[98,99]$ in InGaAs matrix layer lasing at $\sim 1.9 \mu \mathrm{m}$, and subsequent room temperature operation [100]. Later, extensive research in Qdots growth was conducted to achieve high quality InAs Qdots on InP substrate. The active region design developments were carried out on both high index (311)B InP, and exact or vicinal (100) InP substrates. In the following, we review the perfromance achievements in InAs/InP Qdot lasers on these substrates concentrating on (i) InAs/InGaAlAs and (ii) InAs/InGaAsP material systems.
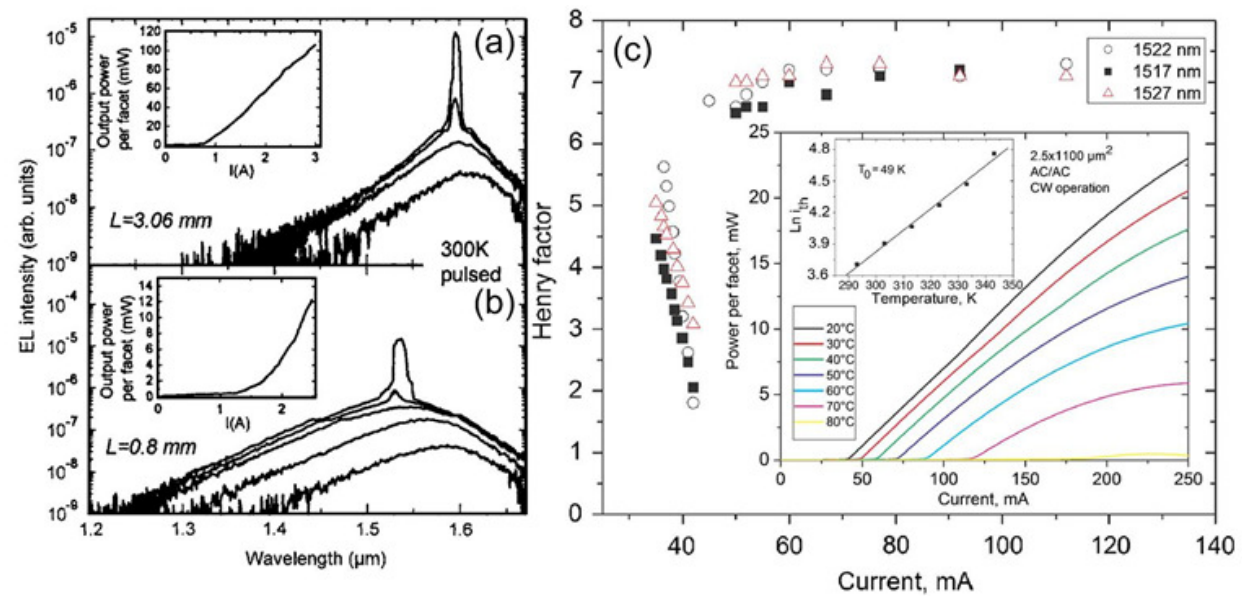

Fig. 16. Room temperature electroluminescence spectra under pulsed operation for severalcurrent densities for a laser cavity length of (a) $3.06 \mathrm{~mm}(65$, 160,208 , and $\left.220 \mathrm{~A} / \mathrm{cm}^{2}\right)$, (b) $0.8 \mathrm{~mm}\left(65,375,1010,1405\right.$, and $\left.1800 \mathrm{~A} / \mathrm{cm}^{2}\right)$. Insets are corres ponding $L-I$ characteristics per facet. (c) Linewidth enhance ment factor(LEF) below and above thre shold of a $1100 \mu \mathrm{m}$ long InAs/InP (311)B InAs/InGaAsP Qdots laser. Inset shows the corresponding $L$ $I$ characteristics w th as-cleaved facets for temperature from 20 to $80^{\circ} \mathrm{C}$ in $\mathrm{CW}$ re gime and characteristic temperature. Taken from [101, 102]

\subsubsection{Lasers on (311)B InP substrate}

\section{In As/InGaAsP material system}

The first $77 \mathrm{~K}$ [100] and room temperature [103] demonstration of long wavelength lasing at $~ 1.4$ $\mu \mathrm{m}$ was reported by Nishi et al. using a 7 stack InAs/InGaAsP Qdots active region on (311)B InP substrate grown by MBE. The lasing action was origin ated from the Qdots ES with threshold current densities $\left(J_{t h}\right) 0.54 \mathrm{kA} / \mathrm{cm}^{2}$ and $4.8 \mathrm{kA} / \mathrm{cm}^{2}$, respectively. The device was based on separate confinement heterostructure $(\mathrm{SCH})$ design with Qdot density $2 \times 10^{10} \mathrm{~cm}^{-2}$. Later, with the improvements in the growth techniques to blue shift the Qdot emission and increase the density and uniformity which improves the laser performance, in particular, the threshold current density, different reports started to appear; from demonstration of optically pumped InAs/InP Qdots laser at 
$1.52 \mu \mathrm{m}[77,104]$ to eventual electrically injected high-gain and low-threshold InAs/InP Qdots laser by Caroff et al. [101]. The latter results are summarized in Figs. 16(a) and (b). By using the double capping technique with a growth interruption under As overpressure, Caroff et al. achieved a Qdot density of $1.1 \times 10^{11} \mathrm{~cm}^{-2}$ and PL linewidth of $50 \mathrm{meV}$ centered at $1.59 \mu \mathrm{m}$ from the laser active region. The threshold current den sity and transparency current density were $190 \mathrm{~A} / \mathrm{cm}^{2}\left(63 \mathrm{~A} / \mathrm{cm}^{2} \mathrm{per}\right.$ layer) and $68 \mathrm{~A} / \mathrm{cm}^{2}\left(23 \mathrm{~A} / \mathrm{cm}^{2}\right.$ per layer), respectively, with GS and ES lasing wavelength at 1.59 $\mu \mathrm{m}$ and $1.53 \mu \mathrm{m}$, respectively. These are the best threshold current density values reported in this material system with high ground-state modal gain of $7 \mathrm{~cm}^{-1}$ per dot layer.

Shortly after this demonstration, employing high quality In As/InGaAsP Qdots, Homeyer et al. [105] reported a record GS modal gain of $8 \mathrm{~cm}^{-1}$ per Qdot layer and best internal quantum efficiency of $62 \%$ from their multi-stack laser devices while maintaining a low threshold current density of $85 \mathrm{~A} / \mathrm{cm}^{2}$ per Qdot layer. The GS and ES lasing were observed at $1.54 \mu \mathrm{m}$ and $1.5 \mu \mathrm{m}$, respectively. The significant improvement in the growth of the material quality was substantiated by: (i) lasing wavelength at $1.52 \mu \mathrm{m}$, originated from a single Qdot active layer on (311)B InP substrate under pulsed current injection [66], (ii) highest reported modal gain of $13 \mathrm{~cm}^{-1}$ from a single stack In As/InP Qdot laser [7, 66], (iii) the largest dot density of $1.3 \times 10^{11} \mathrm{~cm}^{-2}$ and (iv) low internal loss of $6 \mathrm{~cm}^{-1}$ from Qdot lasers on (311)B InP [106]. However, the temperature stability of the In As/InGaAsP Qdots lasers were comparatively poor compared to their InAs/InGaAlAs Qdots laser counterparts, due to the low conduction band offset arising from the mere $25-50 \mathrm{~K}$ characteristics temperature $\mathrm{T}_{0}$ [101]. In general, the output power from the laser devices was reasonable, ranging from few tens (ridge-waveguide laser) to hundreds of $\mathrm{mWs}$ (broad area laser).

Dynamic performance of Qdots lasers on this material system and on (311)B InP substrate was reported by Martinez et al. [102] employing a single mode ridge-waveguide laser structure. GS lasing at $1.52 \mu \mathrm{m}$ and $\mathrm{L}-\mathrm{I}$ characteristics visible up to $75^{\circ} \mathrm{C}$ with a $\mathrm{CW}$ current injection of $>200$ $\mathrm{mA}$ was observed, as depicted in Fig. 16(c). Moreover, measurement of linewidth enhancement factor (LEF) revealed a value of $\sim 1.8$ below threshold before increasing to $\sim 6.8$ above threshold. This was attributed to the band filling of higher energy levels in the wetting layer (see Fig. 16(c)). A maximum relaxation frequency and small signal bandwidth of $3.8 \mathrm{GHz}$ and $4.8 \mathrm{GHz}$, respectively, were also deduced. In general, the lasers static and dynamic performances suggest further improvement of Qdot grown in the material system and on (311)B InP substrate.
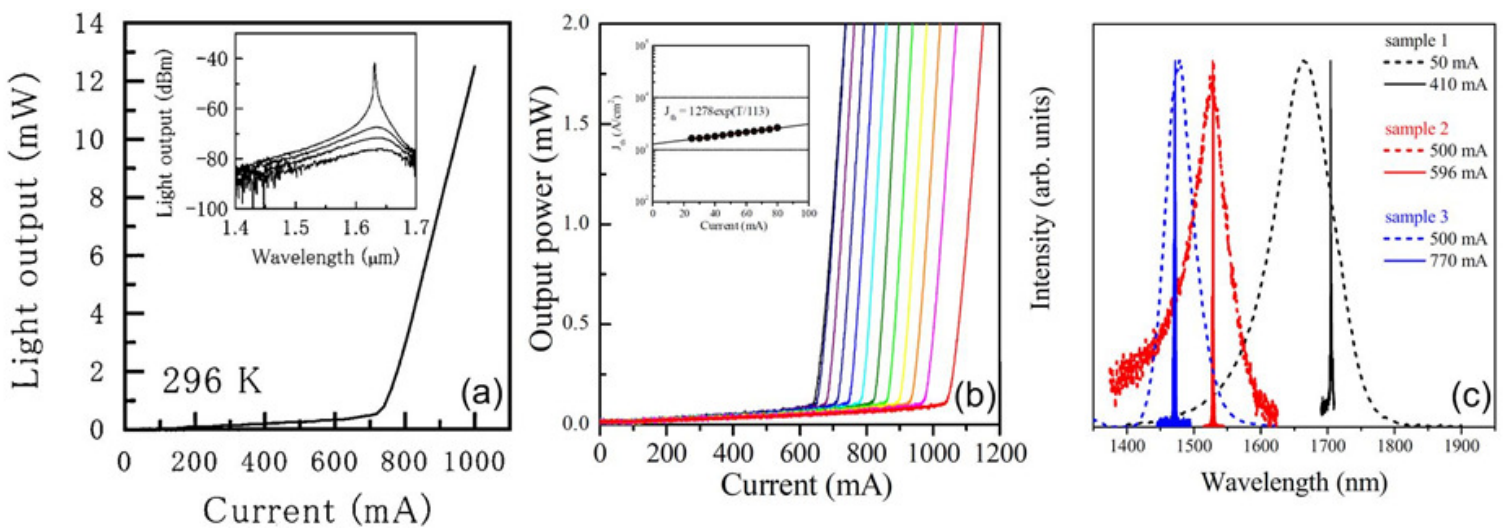

Fig. 17. (a) Light-output curve of an uncoated laser with $2.22 \mathrm{~mm}$ long and $50 \mu \mathrm{m}$ wide stripe at room temperature. Inset is its electroluminescence at 200, 400, 600, and $850 \mathrm{~mA}$. (b) Temperature dependent $\left(25-80^{\circ} \mathrm{C}\right) L-I$ characteristics of InAs/InGaAlAs/(311)B-InP laser comprising $30 \mathrm{InAs}$ Qdots stacks in pulsed mode. Inset shows the relationship between threshold current density and temperature. (c) Electroluminescence and lasing spectra of 30 stacks InAs/InGaAlAs Qdots laser (sample 1, 2, and 3) below (dotted line) and above (solid line) threshold current value and showing the wavelength tuning capability. Adapted from [27, 107, 108].

In As/InGaAlAs Material system

Saito et al. [27] first demonstrated the ground state lasing InAs/InGaAlAs Qdots laser on (311) B InP substrate at a wavelength of $1.63 \mu \mathrm{m}$, and threshold current density of $660 \mathrm{~A} / \mathrm{cm}^{2}\left(132 \mathrm{~A} / \mathrm{cm}^{2}\right.$ per layer) from as-cleaved facets, and $380 \mathrm{~A} / \mathrm{cm}^{2}\left(76 \mathrm{~A} / \mathrm{cm}^{2}\right.$ per layer) from high reflection coated facets, 
plotted in Fig. 17(a). The extracted internal loss was $3.6 \mathrm{~cm}^{-1}$ which to the best of our knowledge the lowest reported value on any InAs/InP Qdots material system. However, after this work, the In As/InGaAlAs Qdot material system on (311) B InP saw a huge gap since most of the research concentrated on the InAs/InGaAsP material system. It was after six years that Alghoraibi et al. [109] and Akahane et al. [35, 110] re-visited this material system on 311(B) InP and reported low temperate and room temperature lasing, respectively, from multi-stack Qdots lasers. A negative characteristics temperature was measured at low temperature (100-130 K) by Alghoraibi et al. which was related to a delayed carrier thermalization within Qdot ensembles. By exploiting the $\mathrm{Al}$ atoms in the spacer layers and employing the strain compensation technique, Akahane et al. demonstrated room temperature lasing from a 30 stack InAs/InGaAlAs Qdots laser with GS lasing at $1.58 \mu \mathrm{m}$ and threshold current density $2.7 \mathrm{kA} / \mathrm{cm}^{2}\left(90 \mathrm{~A} / \mathrm{cm}^{2}\right.$ per layer) from $10 \times 600 \mu \mathrm{m}^{2}$ device, as shown in Fig. 17(b). In addition, by varying the amount of InAs deposition, composition and thickness of the spacer layers, the lasing emission was successfully tuned from 1.47 to $1.7 \mu \mathrm{m} \mathrm{[107]} \mathrm{which} \mathrm{is}$ illustrated in Fig. 17(c). Subsequently, with improvement in the material growth quality, Akahane et al. was able to reduce the threshold current density of the 30 stacked Qdots laser to $1.72 \mathrm{kA} / \mathrm{cm}^{2}$ $\left(57.4 \mathrm{~A} / \mathrm{cm}^{2}\right.$ per layer) with extremely high temperature stability. The corresponding laser characteristics temperature $\mathrm{T}_{0}$ was $114 \mathrm{~K}\left(20-75^{\circ} \mathrm{C}\right)$ and further improved to $148 \mathrm{~K}\left(25-80^{\circ} \mathrm{C}\right)$ from the 20-stack InAs/InP Qdots laser [108]. The threshold current density and $\mathrm{T}_{0}$ values are found to be the best values ever reported on any In As/InP Qdots system. However, high internal loss values of $\sim 26 \mathrm{~cm}^{-1}$ were extracted that was attributed to the imperfect coupling of the optical mode to the multi-stack Qdot gain media.

\subsubsection{Lasers on (100) InP substrate}

\section{$\underline{\text { In As/InGaAlAs material system }}$}

Growth of Qdots on (100) InP substrate was challenging utilizing the InAs/InGaAlAs material system since the conditions always favored Qdash growth instead of Qdots. Consequently, utilizing an assisted growth techniques of a thin GaAs underlying layer before the growth of InAs Qdots on InGaAlAs matrix, Kim et al. demonstrated lasing up to $260 \mathrm{~K}$ [111], and later at room temperature [112], for the first time on a 7-stack Qdot (100) InP laser structure. The achieved threshold current density, Qdot density and lasing wavelength were $2.8 \mathrm{kA} / \mathrm{cm}^{2}$ (400 A/ $\mathrm{cm}^{2}$ per layer), $6.0 \times 10^{10} \mathrm{~cm}^{-2}$ and $1.5 \mu \mathrm{m}$, respectively. Later, the same groups reported room temperature lasing from 7-stacked shape-engineered AGQD Qdots active region with reduced threshold current density of $1.55 \mathrm{kA} / \mathrm{cm}^{2}$ $\left(220 \mathrm{~A} / \mathrm{cm}^{2}\right.$ per layer) [42]. Furthermore, the AGQD laser performance was shown to be better than CQD laser performance which was attributed to the increase in the overlap integral between the electron and hole wave-functions due to two times increase in the aspect ratio (height/width) of the AGQD compared to CQD [113]. More details of the AGQD and CQD Qdot growth was discussed in section 2.1.2.
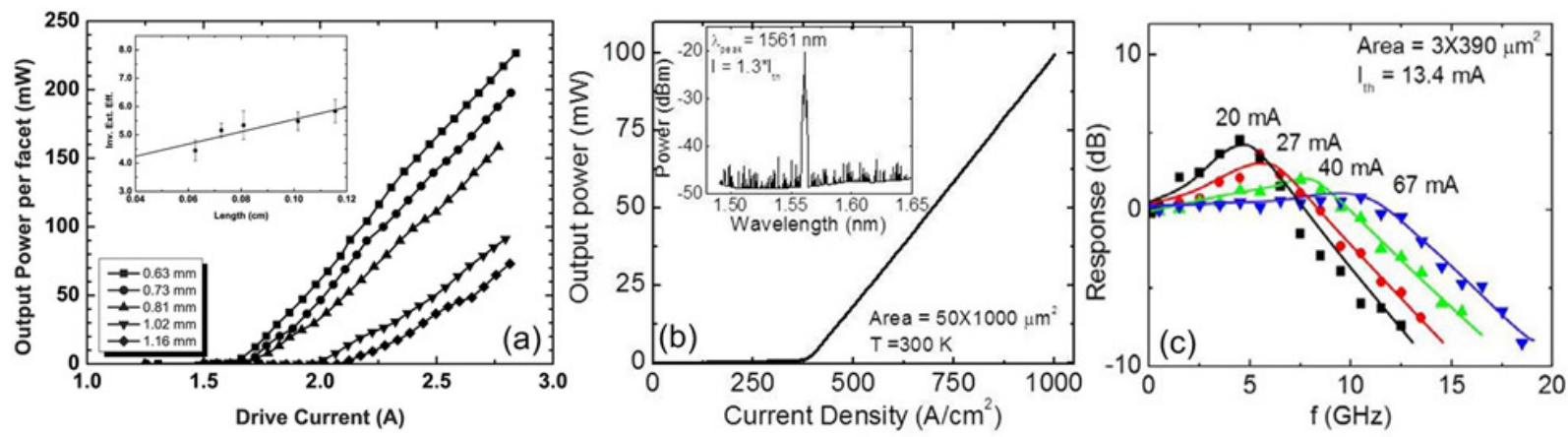

Fig. 18. (a) Plot of the light output power versus drive current of a broad area InAs/InGaAlAs/(100)-InP quantum dot laser at different cavity lengths. The inset depicts the reciprocal external efficiency versus the cavity length providing internal absorption and efficiency. (b) Measured light-current characteristics of a broad area tunnel injection quantum dot laser. Inset shows the lasing spectrum at $\mathrm{I}=1.3 \mathrm{I}_{\mathrm{th}}$. (c) Measured small signal modulation 
response of ridge waveguide tunnel injection quantum dot laser. The solid curves are calculated modulation responses. The $-3 \mathrm{~dB}$ modulation bandw idth measured for $\mathrm{I}=67 \mathrm{~mA}$ is $14.4 \mathrm{GHz}$ Courtesy of $[114,115]$

Recently, Gilfert et al. [114] reported a high gain and room temperature operation of InAs/In GaAlAs Qdots laser on (100) InP by growing in $\mathrm{As}_{2}$ environment using MBE. A low internal loss of $4 \mathrm{~cm}^{-1}$ and high gain of $15 \mathrm{~cm}^{-1}$ per layer were reported, with lasing wavelength at $1.56 \mu \mathrm{m}$ and threshold current density $1.95 \mathrm{kA} / \mathrm{cm}^{2}\left(325 \mathrm{~A} / \mathrm{cm}^{2}\right.$ per layer). The results are plotted in Fig. 18(a). Furthermore, by increasing the material growth quality and reducing the number of Qdots layers, Sichkovskyi et al. [4] reported a record high modal gain of $15.5 \mathrm{~cm}^{-1}$ per layer and an extremely small PL linewidth of $25 \mathrm{meV}$ at $10 \mathrm{~K}$ for their InAs/InP Qdots. The laser also showed high wavelength stability of $0.078 \mathrm{~nm} / \mathrm{K}$ measured above room temperature. The dynamic characteristics of this laser was studied by Gready et al. [116] who reported small signal modulation bandwidth of $5 \mathrm{GHz}$ and large signal modulation capability of $15 \mathrm{Gbps}$ with $4 \mathrm{~dB}$ on/off ratio utilizing pseudo random bit sequence (PRBS). This very different response between the small signal and large signal modulations was attributed to the complex and highly nonlinear carrier dynamics of the In As/InGaAlAs Qdots gain medium. Later, by further optimization of the Qdots gain section [117] a small signal modulation and the large signal modulation bandwidth were pushed to $>9 \mathrm{GHz}$ and 22 Gbps, respectively.

In a very recent demonstration, modulation p-doping and tunneling scheme was applied in the design of InAs/InGaAlAs multi-stack Qdots laser on (100) InP by Bhowmick et al. [115] and the device performance characteristics are plotted in Figs. 18(b) and (c). A low threshold current density of $390 \mathrm{kA} / \mathrm{cm}^{2}\left(78 \mathrm{~A} / \mathrm{cm}^{2}\right.$ per layer), a high characteristic temperature $\mathrm{T}_{0}$ of $100 \mathrm{~K}\left(45-75^{\circ} \mathrm{C}\right)$, near zero below threshold LEF and high modal gain of $14.5 \mathrm{~cm}^{-1}$ per layer was reported, shown in Fig. 18 (b). In addition, a small signal modulation bandwidth of $14.4 \mathrm{GHz}$ was measured from the laser device which is the best value reported on any InAs/InP Qdot material system.

\section{InAs/InGaAsP Material system}

The FP semiconductor lasers investigated with InAs/InGaAsP Qdots on (100) InP were mostly based on $\mathrm{CBE}$ or MOCVD growth techniques until recently, growth using MBE system has also attracted attention. The first demonstration of the electrically injected InAs/In GaAsP/(100)-InP Qdots laser at low temperature $(77 \mathrm{~K})$ was reported on CBE system [118] followed by room temperature operation in both pulsed [119] and CW [120, 121] operations. A pulsed threshold current density of 3.56 $\mathrm{kA} / \mathrm{cm}^{2}$ (713 A/cm $\mathrm{cm}^{2}$ per layer) was reported by Allen et al. [119] who employed Qdots height trimming procedure with growth interruptions under As and As and $\mathrm{P}$ overpressures during the epitaxial growth of the active region. It was shown that higher barrier energy and Qdots densities $\left(1.5 \times 10^{10} \mathrm{~cm}^{-2}\right.$ versus $\left.6.0 \times 10^{11} \mathrm{~cm}^{-2}\right)$ led to a significant improvement in laser threshold current and slope efficiency. Lelarge et al. [121], on the other hand, utilized a hybrid growth technique with MBE grown active region in conjunction with MOCVD grown p-doped cladding and contact layers. With this device growth configuration they demonstrated a CW threshold current density of 1.4 $\mathrm{kA} / \mathrm{cm}^{2}$ (240 A/cm ${ }^{2}$ per layer) and $0.1 \mathrm{~W} / \mathrm{A}$ slope efficiency from a buried ridge-waveguide laser, shown in Fig. 19(a). Lasing action was observed even from the $200 \mu \mathrm{m}$ cavity showing a record high modal gain of $64 \mathrm{~cm}^{-1}\left(10.7 \mathrm{~cm}^{-1}\right.$ per layer), and GS and ES lasing at $\sim 1.5 \mu \mathrm{m}$ and $\sim 1.46 \mu \mathrm{m}$, respectively. As depicted in Fig. 19(b), an internal quantum efficiency and an internal loss of 0.37 W/A and $7 \mathrm{~cm}^{-1}$, respectively, were extracted.

A gradual improvement in the laser performance was observed with the first demonstration of room temperature operation from InAs/InGaAsP Qdots lasers on MOCVD [50, 54, 122] and MBE [71] system, by Notzel et al. and Li et al., respectively. The MOCVD growth laser showed a GS lasing wavelength at $\sim 1.57 \mu \mathrm{m}$ with comparatively reduced threshold current density of $615 \mathrm{~A} / \mathrm{cm}^{2}$ $\left(123 \mathrm{~A} / \mathrm{cm}^{2}\right.$ per layer). The extracted transparency current density and internal loss reached as low as $30 \mathrm{~A} / \mathrm{cm}^{2}$ ( $6 \mathrm{~A} / \mathrm{cm}^{2}$ per layer) and $4.2 \mathrm{~cm}^{-1}$ which are among the best values reported values on (100) InP substrate and in this material system. The results are summarized in in Fig. 19(c). Benefiting from the optimized MOCVD growth process, including reduced As flow rate during Qdots growth 
and utilization of GaAs interlayer, the Qdots uniformity and emission in a multi-stack structure was maintained due to suppression of As/P exchange. On the other hand, MBE grown optimized Qdots laser showed a threshold current density of $790 \mathrm{~A} / \mathrm{cm}^{2}\left(158 \mathrm{~A} / \mathrm{cm}^{2}\right.$ per layer) [71], large temperature stability with $\mathrm{T}_{0}$ of $69 \mathrm{~K}\left(20-70^{\circ} \mathrm{C}\right)$ [123], high wavelength stability of $0.08 \mathrm{~nm} / \mathrm{K}(80-310 \mathrm{~K})$ [124], and lasing around $\sim 1.55 \mu \mathrm{m}$ to $\sim 1.65 \mu \mathrm{m}$. A small LEF of $\sim 1.4-1.6$ above threshold and $<1$ below threshold, was measured recently by Jiao et al. [125] which is half the value reported by Lelarge et al. ( 2.2 below threshold) [121], and the Qdots exhibited better uniformity with PL linewidth 63 $\mathrm{meV}$ at room temperature. Laser devices at low temperature [56] and room temperature [55, 126] lasing from InGaAs Qdots on InGaAsP/(100)-InP were also reported with high Qdot density reaching value $1.1 \times 10^{11} \mathrm{~cm}^{-2}$ and with notable performances.

Qdot lasers based on In As/InGaAsP DoWELL [74] and InAs/In As dot-on-well [127] active region design were also demonstrated after observation of their superior quality at material level which has been discussed in section 2.2.1. As expected, the laser devices employing these structures showed improved performance attributed to the efficient carrier capturing process. Employing doton-well structure Kotani et al. [127] reported GS lasing at $1.74 \mu \mathrm{m}$ from a single Qdot stack with threshold current density $2.75 \mathrm{kA} / \mathrm{cm}^{2}$ and improved slope efficiency compared to a 5-stack Qdot laser structure. Furthermore, using an optimized growth conditions of the waveguide core and claddings and with minimum defect density, longest ever reported GS lasing wavelength of $1.95 \mu \mathrm{m}$ from a single stack InAs/InP Qdot-on-well laser was demonstrated with an extremely low threshold current density of $100 \mathrm{~A} / \mathrm{cm}^{2}$ [128] which is plotted in Figs. 19(d) and (e). In general, the performance of InAs/InP Qdot lasers showed tremendous improvement in the past decade and is expected to be comparable to the performance of the InAs/GaAs Qdots lasers very soon. which have already achieved a threshold current density of $17 \mathrm{~A} / \mathrm{cm}^{2}$ [2].
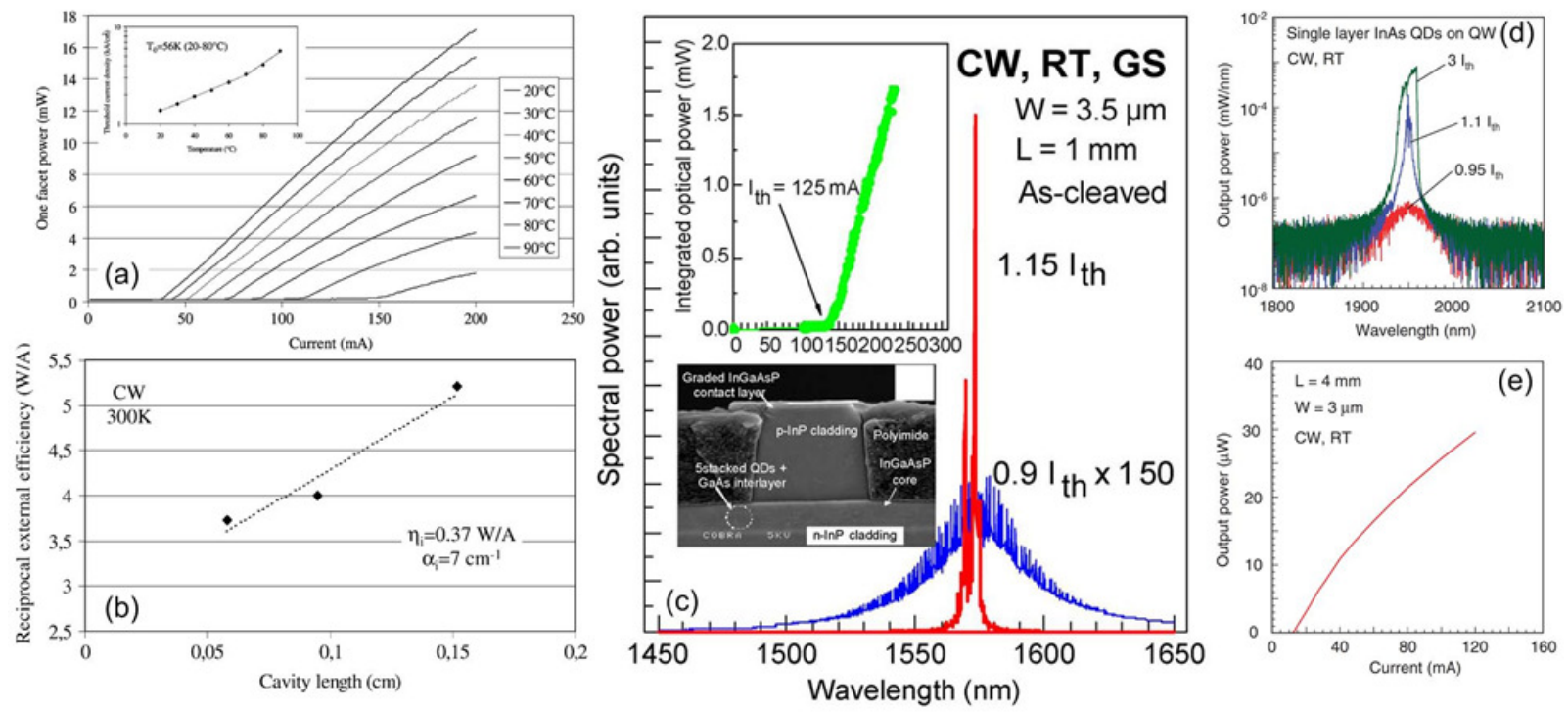

Fig. 19. (a) Output power versus injection current of a $2.5 \times 1040 \mu \mathrm{m}^{2}$ buried ridge-waveguide InAs/InGaAsP/(100)-InP Qdot laser measured under CW operation in the temperature range of $20-90{ }^{\circ} \mathrm{C}$. Inset shows the threshold current density versus temperature. (b) Reciprocal external differential efficiency of buried ridge-waveguide laser lasers versus cavity length measured under CW room temperature operation. (c) Amplified spontaneous emission and lasing spectra (CW, room temperature, Qdot GS) together with the light-current characteristics (upper inset) of the InAs/InGaAsP/(100)InP Qdots laser shown in the scanning electron microscopy image (lower inset) (c) Amplified spontaneous emission and lasing spectra and (d) light output versus injection c urrent curve of a single-layer InAs/InP Qdot-on-well laser emitting near 2.0 $\mu$ m. Reproduc ed from [121, 128, 129].

\subsubsection{Multiwavelength lasers}

Application of InAs/InGaAsP/(100)-InP Qdots gain chip in conjunction with the external cavity laser system (ECLS) in Littrow configuration was first reported by Allen et al. [119, 130]. In this system, a single lasing mode can be tuned at a special angle of blazed gratings. A broad electroluminescence profile of $175 \mathrm{~nm}$ was achieved before lasing from the ridge waveguide laser device and when used 
in the ELCS, a tuning range as large as $110 \mathrm{~nm}$ was demonstrated. Shorter cavity laser diodes showed broader wavelength tunability than longer cavity diodes but at the expense of high threshold current density. The internal loss of the system was estimated to be $20-30 \mathrm{~cm}^{-1}$ [131]. In the same year, Ortner et al. [132] showed improved tunability from the InAs/InP Qdots ECLS with a value of $166 \mathrm{~nm}$, illustrated in Fig. 20(a). The key idea was to coat a high reflection layer on one facet and antireflection coating on the other facet. Under pulsed operation, an output power as high as $6 \mu \mathrm{W}$ was obtained when tuned at $1.571 \mu \mathrm{m}$ wavelength. The threshold current density was found to increase at the extreme gratings angles which was attributed to the decrease in the Qdots material at extreme short (higher density of states but decrease in the occupational probability at a given current injection), and long (reduced density of states) wavelengths. A high output power up to $24 \mathrm{~mW}$ at a tuned wavelength of $\sim 1.594 \mu \mathrm{m}$ in the ECLS was reported by Chen et al. [133] on the $8 \times 1500 \mu \mathrm{m}^{2}$ In As/InGaAsP on (100) InP Qdots laser. A maximum tunable range of $70 \mathrm{~nm}$ was obtained with a threshold current density $<1.62 \mathrm{kA} / \mathrm{cm}^{2}$. By utilizing smaller device size, the tuning range was increased to $98 \mathrm{~nm}$ but at the expense of higher threshold current density of $3.33 \mathrm{kA} / \mathrm{cm}^{2}$. This is shown in Figs. 20(b) and (c). A more detailed review on the ECLS on InAs/InP Qdots lasers was performed by Li et al. [131].

In the lasing mode, the large gain bandwidth and Qdots gain saturation usually results in broadening of the lasing spectra with increasing current injection. The resulting spectra could be very wide and flat-topped. This feature was exploited to realize multiwavelength laser where the sharp line corresponding to the FP modes represents a channel with spacing determined by the cavity length. Such devices are highly attractive in optical networks replacing many individual lasers. This concept was first reported by Liu et al. [134] on In As/InP Qdots material system. By employing a 2 $\times 4500 \mu^{2}$ ridge-waveguide InAs/InGaAsP/(100)-InP Qdots laser and in CW operation, 93 uniform channels (centered at $1.642 \mu \mathrm{m}$ ) with channel intensity non-uniformity of $3 \mathrm{~dB}$ was reported. The channel spacing was estimated to be $86 \mathrm{pm}(9.56 \mathrm{GHz})$ with $\sim 30 \mu \mathrm{W}$ output power from each channel. It was suggested that the non-trivial channel spacing alteration due to intracavity waveguide dispersion (alteration in temperature, effective current injection, and vacuu m wavelength) of $\pm 0.8 \mathrm{pm}$ could be corrected by designing the dispersion of ridge-waveguides. In a subsequent study, Liu et al. [135] estimated the linear intracavity waveguide dispersion of $-5.1 \times 10^{-4} \mathrm{~nm}^{-1}$ from a $2 \times 461 \mu \mathrm{m}^{2}$ ridge-waveguide laser with 24 channels. The channel spacing and maximum signal-to-noise ratio was $0.8 \mathrm{~nm}(91.6 \mathrm{GHz})$ and $62 \mathrm{~dB}$, respectively. Very recently, Lu et al. [136] developed a $50 \mathrm{GHz}$ spacing C-band (central wavelength $1.555 \mu \mathrm{m}$ ) multiwavelength laser using a $3 \times 860 \mu \mathrm{m}^{2}$ ridgewaveguide In As/InP Qdot active region device with an average output power of $40 \mathrm{~mW}$ that matches the International Telecommunication Union (ITU) grid, as shown in Fig. 21(a). The relative intensity noise (RIN) of individual channel which describes the laser's maximum available amplitude range for signal modulation, was measured to be $\sim-118 \mathrm{~dB} / \mathrm{Hz}[69]$.
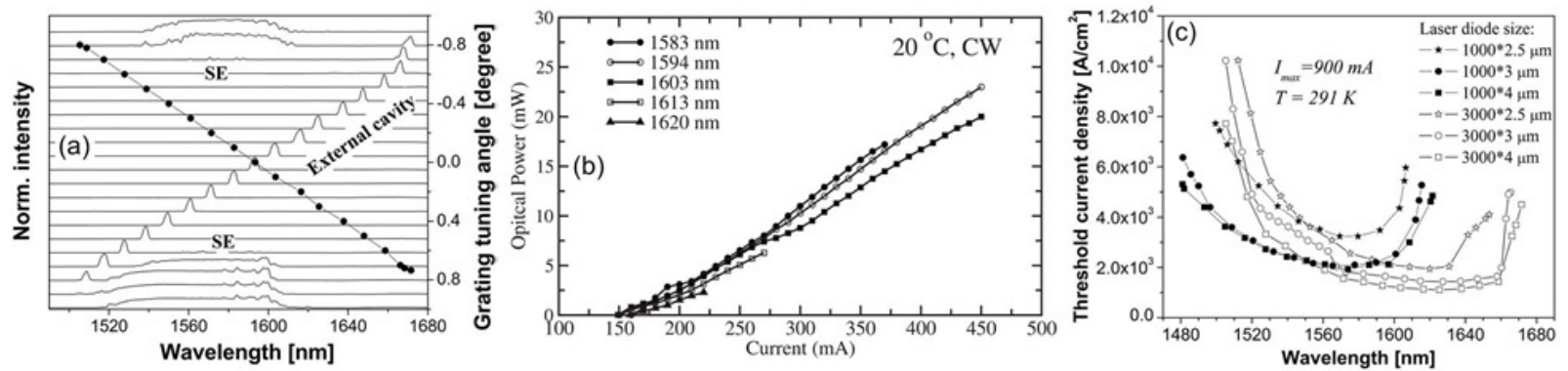

Fig. 20. Spectra of the ECLS displaying the peak linearly tuned (full circles) in wavelength with the angular position of the blazed grating. The InAs/InGaAsP Qdots laser diode size is $4 \times 3000 \mu \mathrm{m}^{2}$. Note that simultaneous spontaneous emission of the QD ensemble also emerges. (b) $L-I$ curves of $8 \times 1500 \mu \mathrm{m}^{2}$ InAs/InP Qdots laser in ECLS configuration at different operating wavelengths. (c) Threshold current density as a function of laser wavelength for different de vice lengths and widths as denoted by the different symbols. An upper limit for the injected current has been set to $900 \mathrm{~mA}$ to avoid damaging the haser diode. Adapted from [130, 132, 133].

\subsubsection{Single mode lasers}


Single mode lasers are the opposite extreme to the broad gain multiwavelength lasers, and forms the backbone of the long haul optical communications. The first single mode InAs/InP Qdots laser diode was demonstrated by Kim et al. [137] on InAs/InGaAlAs material system and using distributedfeedback (DFB) configuration. The room temperature lasing emission of $3 \times 1000 \mu^{2}$ ridgewaveguide device was $1.564 \mu \mathrm{m}$ with side mode suppression ratio (SMSR) of $42 \mathrm{~dB}$ at $100 \mathrm{~mA} \mathrm{CW}$ current injection. However, the output power of the device was low reaching a value of $1 \mathrm{~mW}$ at 100 $\mathrm{mA}$ and a red shift in the Qdot lasing emission was observed compared to the Qdot lasing wavelength without the gratings. This required tuning of the Qdots emission by reducing the amount of InAs deposition for the Qdot self-assembled formation. On the other hand, Lu et al. [138] reported a InAs/InGaAsP/(100)-InP Qdots DFB laser fabricated using the commercial buried grating process. After the growth of the active region using CBE system, the wafer was removed to pattern the grating region and then the p-contact layers were regrown using MOCVD system. No change in the PL emission was observed before and after grating overgrowth. The fabricated $3 \times 1000 \mu \mathrm{m}^{2}$ ridgewaveguide DFB laser demonstrated a lasing threshold of $48 \mathrm{~mA}$ with output power up to $19 \mathrm{~mW}$ at $200 \mathrm{~mA} \mathrm{CW}$ operation up to $90^{\circ} \mathrm{C}$. The lasing wavelength was $1.52 \mu \mathrm{m}$ with SMSR of $>61 \mathrm{~dB}$ and is depicted in Fig. 21(b). The measured RIN and optical linewidth was around $<-154 \mathrm{~dB} / \mathrm{Hz}(10$ $\mathrm{MHz}-10 \mathrm{GHz}$ ) and $150 \mathrm{kHz}$, respectively.

Recently, Reithmaier et al. [139] modified the InAs/InGaAlAs/(100)-InP Qdots layer design by integrating a In GaAsP etch stop layer which allowed fabrication of the lateral gratings after ridgewaveguide processing. An output power of $>12 \mathrm{~mW}$ in $\mathrm{CW}$ operation, lasing at $\sim 1.52 \mu \mathrm{m}$ with SMSR of $45-50 \mathrm{~dB}$ was reported from the $2 \times 1000 \mu \mathrm{m}^{2}$ device at room temperature. The results are shown in Fig. 21(c). The optical linewidth in this case was measured to be $\sim 149 \mathrm{kHz}$, however, due to internal heating, the lasing wavelength shifted with increasing current injection.
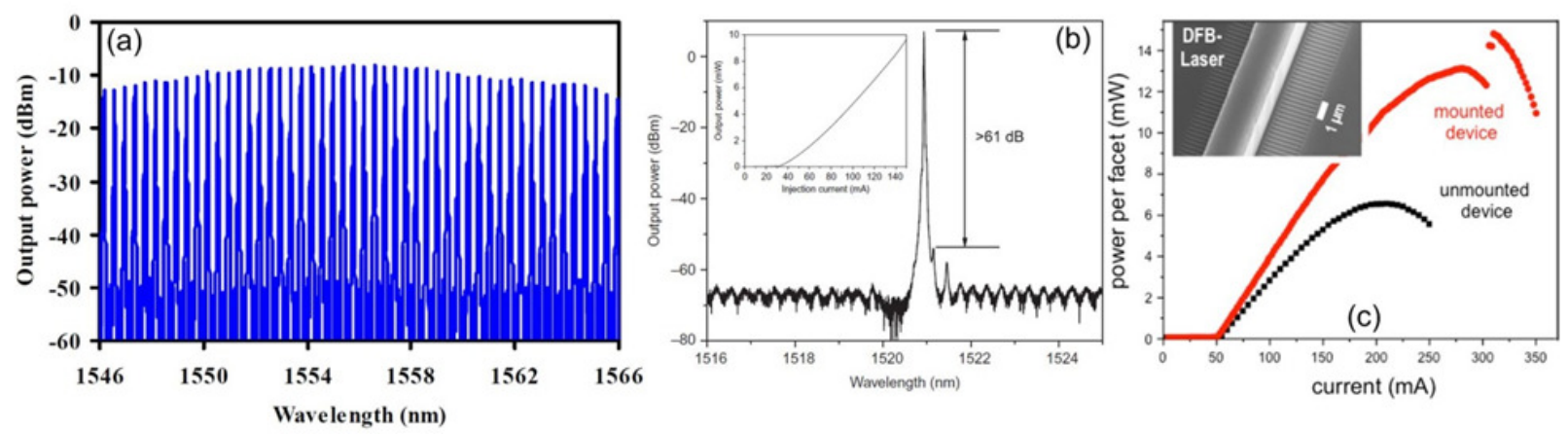

Fig. 21. (a) The optical spectra of over 46 channels InAs/InGaAsP/InP Qdots multiwa velength laser with 50-GHz frequency spacing and within the 3$\mathrm{dB}$ bandwidth at an injection current of $200 \mathrm{~mA}$. (b) Lasing spectrum of single mode InAs/InGaAsP/InP Qdots DFB laser at an injection current of 200 $\mathrm{mA}$ and at room temperature. Inset shows the corresponding $L-I$ curve of the ridge-waveguide InAs/InP DFB laser operating in CW at room temperature. (c) $L-I$ curve of an un-mounted and mounted InAs/InGaAlAs/InP Qdots DFB laser. The inset shows a scanning electron microscopy (SEM) picture of the lateral first order grating before planarization and contact metallization. Taken from [136, 138, 139]

\subsection{InAs/InP Qdots mode-locked lasers}

Mode synchronization in FP lasers could emit very short pulses with the repetition rate determined by the round trip tracing of the cavity. This concept of mode locking in laser devices could be key components in optical clocking, optical communications, etc. Moreover, monolithic passive mode locking device is highly attractive because of its compactness, stability and straightforward fabrication process. In this case, two-section devices are usually employed with one section being forward bias which forms an optical amplifier, and the other is reversed bias which forms a saturable absorber (SA) section and assists in achieving the mode synchronization condition [2]. Since, selfassembled Qdots possess broad gain due to inhomogeneous broadening; the requirement of mode synchronization could be easily fulfilled from the same array of Qdots (some Qdots act as absorbers while others contribute to gain) in a mono-section device known as self-pulsating devices [2]. Here, we reviewed the achievements of mode locking in InAs/InP Qdots laser utilizing both of these configurations. 


\subsubsection{Two section}

Heck et al. [140] reported the first investigation of mode-locking characteristics from two section In As/InGaAsP Qdots laser on (100) InP. The long $2 \times 9000 \mu \mathrm{m}^{2}$ ridge-waveguide device with $270 \mu \mathrm{m}$ SA section showed a threshold current of 660-690 mA and demonstrated a clear mode locking characteristics with strong peaks in the radio-frequency (RF) spectrum at a repetition rate of $4.6 \mathrm{GHz}$ (round trip cavity frequency) and peak width of $0.57 \mathrm{MHz}$ at $-20 \mathrm{~dB}$, as shown in Fig. 22(a). The pulse duration was measured to be around 6-11 ps. On the other hand, the smaller device $(2 \times 7000$ $\mu \mathrm{m}^{2}$ ridge-waveguide with $350 \mu \mathrm{m}$ SA section) did not show the signs of model-locking but instead showed Q-switching at three regimes 32.5, 153, and $390 \mathrm{MHz}$, with increasing injection, as depicted in Fig. 22(b). The pulse duration was $0.6-0.8$ ps. A reverse bias of $-1 \mathrm{~V}$ and $-3 \mathrm{~V}$ were applied to long and short devices, respectively. Subsequently, the timing jitter was evaluated for the long device with a value 35-39 ps and the effect of longer SA of $540 \mu \mathrm{m}$ was also investigated [141]. It was found that mode locking was achieved at relatively high values of injection current and higher timing jitter 36-53 ps, with output power, in the regime of stable mode locking, in between 1-4 mW. Moreover, from a $2 \times 4000 \mu \mathrm{m}^{2}$ ridge-waveguide with 120 and $240 \mu \mathrm{m}$ SA, stable passive modelocking was observed for a smaller regime with pulse repetition rate of $10.5 \mathrm{GHz}$ corresponding to the 95 ps cavity round trip. The RF peak wid th was in the order to $0.5-2.5 \mathrm{MHz}$ at $-20 \mathrm{~dB}$. The effect of simultaneous lasing from ground state and excited state in the short cavity laser on mode locking characteristics was also studied by Heck et al. [141]. It was found that not only mode-locking of both the mode groups was observed but also their pulses were synchronized. A similar mode-locking characteristic was also observed by Tahvili et al. [142] in a dual-wavelength mode-locked laser, which corresponds to lasing emission from the same electronic transition of a $2 \times 4000 \mu^{2}$ twosection device. In addition, employing a long $13 \mathrm{~mm}$ device $(650 \mu \mathrm{m}$ long SA), a stable mode locking of $3 \mathrm{GHz}$ was also demonstrated which is the lowest repetition rate reported for a monolithic In As/InP Qdots laser.

Recently, hybrid mode-locking in two section In As/InGaAsP/(100)-InP Qdots laser was in vestigated $[142,143]$. In this case an electrical modulation, with frequency close to the cavity freespectral range, is added to a reversely biased SA. Employing a $9000 \mu \mathrm{m}$ two-section device, it was shown that the timing jitter strongly decreased to $0.5-0.6 \mathrm{ps}(10 \mathrm{kHz}-300 \mathrm{MHz})$ and via second order dispersion of single mode fiber, pulse compression down to 2 ps was obtained at $4.6 \mathrm{GHz}$ pulse frequency. In the case of $13 \mathrm{~mm}$ device, the timing jitter reduced from 8-16 ps (passive modelocking) to $0.7-3$ ps (hybrid mode-locking).
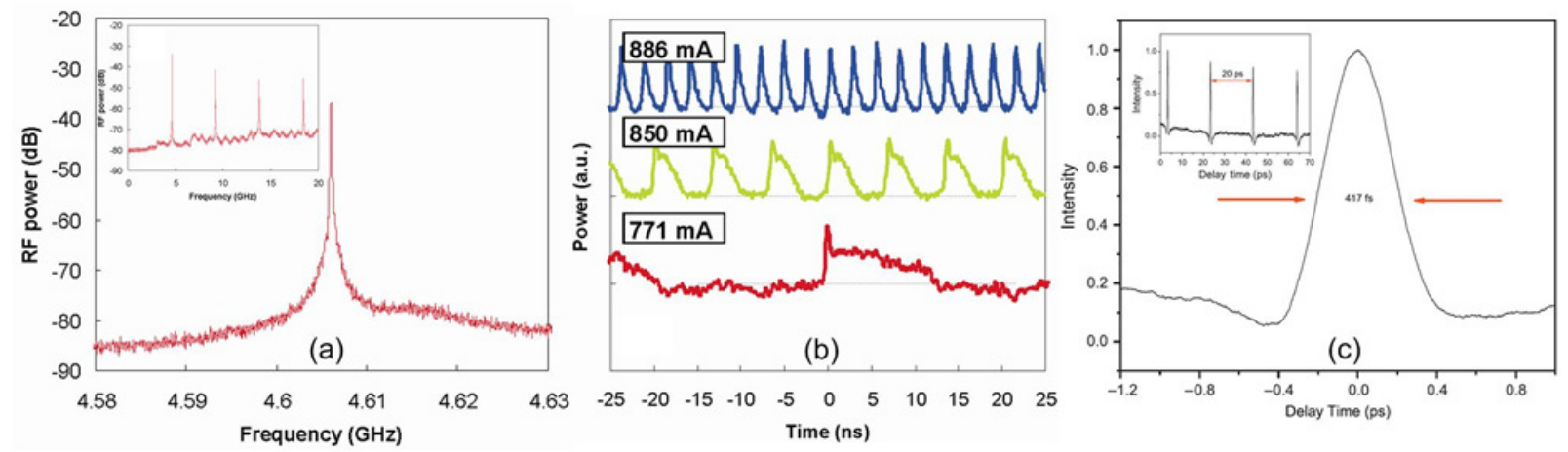

Fig. 22. (a) Detailed view of the spectrum around the first RF peak obtained for a $9 \mathrm{~mm}$ device with $3 \% \mathrm{SA}$ length. Injection current is $900 \mathrm{~mA}$ and $\mathrm{SA}$ bias voltage is $-1 \mathrm{~V}$. Inset shows the RF-spectrum. (a) Oscilloscope traces obtained for different injection currents corresponding to the three different regimes of Q-switching obtained with a $7 \mathrm{~mm}$ de vice with $5 \% \mathrm{SA}$ length and an SA bias voltage of $-3 \mathrm{~V}$. Traces have been offset for clarity, i.e. the dotted lines represent the respective 0-levels. (c) Optical intensity autocorrelation trace with second-order autocorrelation measurements. Assuming a Gaussian pulse shape, the real pulse duration of the $2.5 \times 861 \mu \mathrm{m}^{2} \mathrm{InAs} / \mathrm{InP}$ Qdots mode-locked laser was estimate d at $295 \mathrm{fs}$ when the injection current is $200 \mathrm{~mA}$ and at a temperature of $18^{\circ} \mathrm{C}$. Inset shows the optical intensity autocorrelation pulse trains with the periodic time of $20 \mathrm{ps,}$, which corresponds to a repetition rate of $50 \mathrm{GHz}$. Courtesy from [11, 140, 144] 


\subsubsection{Self-pulsation}

Monosection or single-section mode-locking was first demonstrated by Renaudier et al. [120] on InAs/InP Qdots/Qdash active region laser. Self-pulsation at $45 \mathrm{GHz}$ under $\mathrm{CW}$ operation was observed from a $950 \mu \mathrm{m}$ device which is equivalent to the round trip cavity time. From the mode beating spectra, the pulse width was measured to be $70 \mathrm{kHz}$ at $-3 \mathrm{~dB}$, operating at $160 \mathrm{~mA}$. Active mode-locking with an optical clock signal at $45.06 \mathrm{GHz}$ was also reported with a significant decrease in the RF spectral linewidth to $3 \mathrm{KHz}$ (determined by the chosen resolution bandwidth) and extremely low timing jitter of $82 \mathrm{fs}$. This work was soon followed up with the demonstration of 92 $\mathrm{GHz}$ repetition rate with femtosecond pulses from passive-single section CBE grown $2.5 \times 456 \mu \mathrm{m}^{2}$ InAs/In GaAsP Qdot laser on (100) InP, lasing at $~ 1.54 \mu \mathrm{m}$ (C-Band), by Lu et al. [145] and dualwavelength self-mode locking at $92.5 \mathrm{GHz}$ by Liu et al. [146]. In the former case, the laser showed a threshold current of $17.2 \mathrm{~mA}$ under $\mathrm{CW}$ operation with output power $\sim 9 \mathrm{~mW}$ at $45 \mathrm{~mA}$. Moreover, through autocorrelation measurements, periodic time of the measured pulse train was 10.87 ps (92 $\mathrm{GHz}$ ) with 312 fs pulse width (after removing the DC components from $442 \mathrm{fs}$ autocorrelation trace and converting to real pulse duration), at $45 \mathrm{~mA}$. This self-pulsation characteristic from Qdot active media was attributed to strong self-phase and cross-phase modulation effects leading to significant enhancement in the four wave mixing (FWM) process between the longitudinal laser modes of the FP laser cavity. Subsequently, an L-band $(\sim 1.59 \mu \mathrm{m})$ mono-section InAs/InP Qdots mode-locked laser was reported with a $3 \times 930 \mu \mathrm{m}^{2}$ (with one facet high reflection coated) device exhibiting 46 $\mathrm{GHz}$ RF resonance peak with linewidth $<100 \mathrm{kHz}$ and optical signal-to-noise-ratio $>20 \mathrm{~dB}$ [147]. The measured autocorrelation pulsed width was $629 \mathrm{fs}$ which after conversion to real pulse width was $445 \mathrm{fs}$. The time bandwidth product of this device was 0.69 which was larger than the transformlimited Gaussian-shaped pulses of 0.44 and was attributed to residual frequency chirp being present in the pulses. With continues improvements in the Qdots active region design, material quality, and fabrication, $\mathrm{Lu}$ et al. reported a real pulse duration of $295 \mathrm{fs}$ from $2.5 \times 861 \mu \mathrm{m}^{2}$ laser device at a repetition rate of $50 \mathrm{GHz}$ and lasing around $\sim 1.56 \mu \mathrm{m}$ with an output power of $40 \mathrm{~mW}$ at $200 \mathrm{~mA}$. The results are shown in Fig. 22(c). This is the smallest pulse width value reported on InAs/InP Qdots material system [11, 144] without any external means. In addition, achievement of repetition rates as low as $10 \mathrm{GHz}$ and as high as $100 \mathrm{GHz}$ from $2.5 \times 430 \mu \mathrm{m}^{2}$ and $2.5 \times 4300 \mu \mathrm{m}^{2}$ Qdot modelocked lasers, respectively, highlights the achievements of In As/InP Qdot active media in mode locking. Very recently, apart from the leading (100)InP based Qdot mode-locked lasers, selfpulsation in InAs/InGaAsP Qdots lasers on (311)B InP substrate was also reported by Klaime et al. [106] with repetition rates around 23 and $39 \mathrm{GHz}$ and pulse width down to $1.5 \mathrm{ps}$. The $2 \times 1000 \mu \mathrm{m}^{2}$ and $2 \times 2000 \mu \mathrm{m}^{2}$ ridge-waveguide devices exhibited extremely low RF spectral width of $20 \mathrm{kHz}$ indicating very low timing jitter.

Through changing the inherent active length of the FP cavity to external cavities coupled with fiber Bragg gratings (FBG), Lu et al. [11] was able to generate a pulse repetition rate of 437 $\mathrm{GHz}$, thus removing the barrier which limits the realization of very high repetition rates (due to cavity length dependence) mode locking. In this case, eight FBG with equal frequency spacing were utilized to form the external fiber mixed cavity with $2.5 \times 861 \mu \mathrm{m}^{2} \mathrm{InAs} / \mathrm{InGaAsP} /(100)$-InP Qdots laser with one fact high reflection coated and other anti-reflection coated. The working principle was based on the reflection of those longitudinal modes which matches the wavelength of FBG, by the external cavity, thus increasing their intensities compared to other longitudinal modes of the laser itself ( -40 to $-10 \mathrm{dBm}$ higher and equally spaced). By using an autocorrelator, a pulse train of $2.29 \mathrm{ps}$ duration ( $810 \mathrm{fs}$ real width) was measured at $437 \mathrm{GHz}$, with an average output power of $1 \mathrm{~mW}$. This work was further improved by demonstration of $403 \mathrm{GHz}$ pulse repetition rate with a pulse width of $268 \mathrm{fs}$ at $110 \mathrm{~mA}$ and $563 \mathrm{fs}$ at $112 \mathrm{~mA} \mathrm{CW}$ current injection from a similar FBG based external cavity configuration and utilizing $3 \times 850 \mu \mathrm{m}^{2}$ Qdot laser device (with free spectral range $50.25 \mathrm{GHz}$ ) in the C-band [148]. This is illustrated in Figs. 23(a) and (b) and was attributed to the FWM between the strong light peaks of the optical spectrum (obtained in external cavity configuration) and leading to mode-locking. 

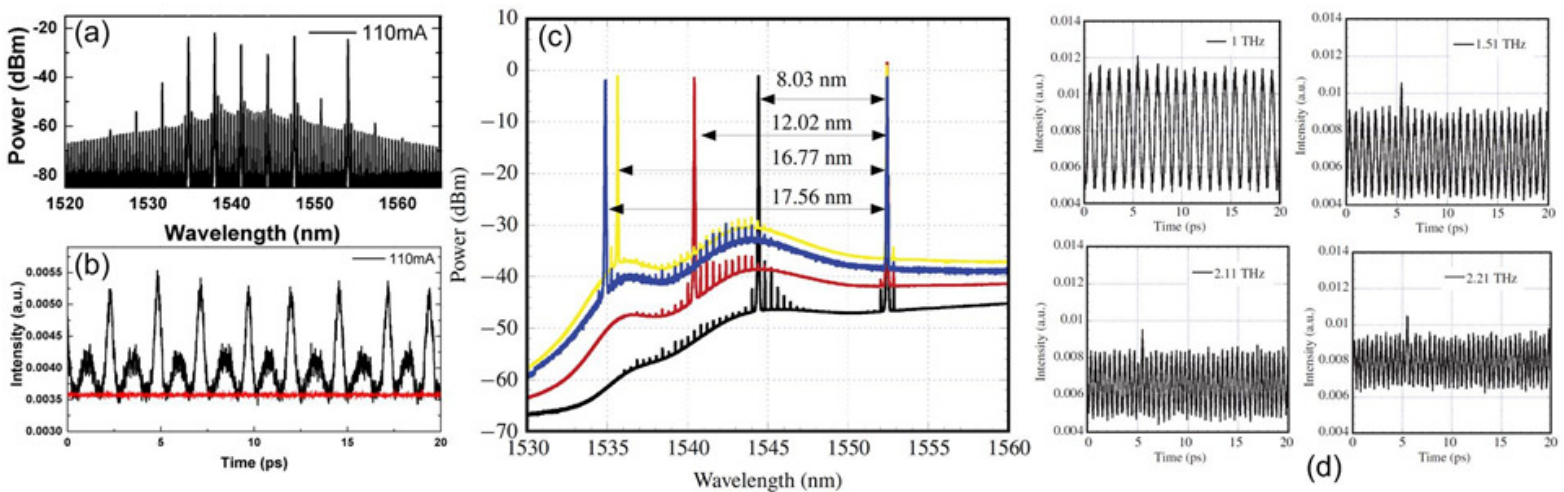

Fig. 23. (a) Measured optical spectrum of the InAs/InP Qdots mode-locked laser with the external cavities of eight FBGs, at the biased current of 110 $\mathrm{mA}$. Optical resolution use d is $10 \mathrm{pm}$. (b) Measured autocorrelations of the pulse train indicating a period of $2.48 \mathrm{ps}$, which corresponds to a repetition rate of $403 \mathrm{GHz}$ at the biased current of $110 \mathrm{~mA}$. The red line indicates noise level of the autoc orrelator. The intensity autocorrelation is measured based on second-harmonic generation with a time resolution of $10 \mathrm{fs}$. (c) Tunable dual-mode spectra of InAs/InP Qdots laser with external cavities of three FBGs, from 1 to $2.21 \mathrm{THz}$ at the biased current of $120 \mathrm{~mA}$ and temperature controlled at $18{ }^{\circ} \mathrm{C}$. The mode separations are indicated in the spectrum. (d) Tunable beating frequencies from 1 to $2.21 \mathrm{THz}$ in the time domain corresponding to the four mode-separations in (c). Taken from [148, 149].

The accomplishment of coupled external cavity In As/InP Qdots laser based mode locking was brought to the next level by the demonstration of pulse repetition rates in terahertz range by selection of inter frequency spacing of FBG in the terahertz since this spacing determines the repetition rate. A $3 \times 1000 \mu \mathrm{m}^{2} \mathrm{InAs} / \mathrm{InP}$ Qdot laser with one facet $95 \%$ high reflectivity and other $31 \%$ reflectivity was utilized with a FBG central wavelengths corresponding to $1.01 \mathrm{THz}$ [10]. It was shown that at low CW bias current below $48 \mathrm{~mA}$, the coupled cavity modes lased with dominant wavelength peaks defined by the three FBG wavelengths. The resulting pulsed train measured with autocorrelator showed pulsed repetition rate of $1.0 \mathrm{THz}$ with pulse duration of $500 \mathrm{fs}$. However, with increasing bias the envelope of $1.01 \mathrm{THz}$ pulse train got modulated at $43.8 \mathrm{GHz}$ equal to the mode spacing of the Qdots laser itself which dominated with further increase in bias current. Modifying the external coupled cavity Qdots laser configuration by employing two FBG configurations with one FBG tunable in wavelength, Jiao et al. demonstrated a tunable 1 to $2.21 \mathrm{THz}$ ultra-high repetition rate mode-locked laser; the highest repetition rate reported on a InAs/InP Qdots material system [149]. This is illustrated in Fig. 23(c) by plotting the mode spectra and in Fig. 23(d) by showing the corresponding pulse widths variation from $158 \mathrm{fs}$ (at $2.11 \mathrm{THz})$ to $315 \mathrm{fs}$ (at $1.0 \mathrm{THz}$ ). These are the record pulse width values ever reported on InAs/InP Qdots material system. Mode locking via external cavity configuration seems to be a promising technique in achieving extremely high repetition rates which is FBG dependent rather than Qdots laser except that the gain and carrier dynamics of the active media should be fast enough to sustain successive pulse emission.

\subsection{InAs/InP Qdots semiconductor optical amplifiers}

Long wavelength SOA based on InAs/InP Qdots active region has shown remarkable improvement in terms of performance characteristics compared to the Qwell based SOA [150-152], particularly broadband gain profile. This is attributed to the inhomogeneously broadened Qdots gain and the ultra-fast carrier capture process [153]. In the subsequent sub-sections, we review the recent achievements of InAs/InP Qdot based SOA component operating around $\sim 1.55 \mu \mathrm{m}$ and encompassing $\mathrm{C}-\mathrm{L}-\mathrm{U}$ communication bands of the ITU grid.

\subsubsection{Broad Gain}

Akiyama et al. [154, 155] demonstrated an InAs/InGaAsP on (100) InP MOCVD grown Qdots SOA with more than $25 \mathrm{~dB}$ gain over $90 \mathrm{~nm}$ bandwidth and noise figure $(\mathrm{NF})<5 \mathrm{~dB}$ which is the degradation of the signal due to amplified-spontaneous emission noise. The saturated output power of this $2.2 \times 6150 \mu \mathrm{m}^{2}$ long device was measured to be $>19 \mathrm{dBm}$ within the bandwidth. Such an impressive results, which are summarized in Fig. 24(a) and (b), has not been achieved by the Qwell 
couparts thus making InAs/InP Qdots SOA highly attractive in this domain. The polarization dependence of the gain was eliminated by growing closely stacked columnar Qdots, thus favouring vertical coupling and leading to polarization insesitive SOA gain characteristics [156]. This work was soon followed by various deomstrations of the gain characteritics of InAs/InP Qdots SOA [157, 158]. For instance Kim et al. [159] showed a chip gain as high as $37 \mathrm{~dB}$ and maximum saturation output power $17.2 \mathrm{dBm}$ at $950 \mathrm{~mA}$ from $3 \times 3000 \mu \mathrm{m}^{2}$ long device after determining the coupling-loss $(14.4 \mathrm{~dB})$ and performing the fiber-to-fiber gain $(22.5 \mathrm{~dB})$ measurements. The gain peak was found to be at $\sim 1.51 \mu \mathrm{m}$ with a NF of 7.2-9 dB. Contestabile et al. [160] obtained fiber-to-fiber peak gain of $37 \mathrm{~dB}$ at $1.49 \mu \mathrm{m}$ with $3 \mathrm{~dB}$ bandiwdth of $75 \mathrm{~nm}$. A maximum output saturation power measured was around $18.5 \mathrm{dBm}$. These impressive values are attributed, in general, to the spatially distributed Qdots optical tranistions, ultra-short gain recovery time (discussed later in this section), and small optical confinement factor [69].

\subsubsection{Ultrafast gain and index dynamics}

Ultrafast gain response, which is in the order ot few picoseconds, is the characteristics property of Qdots, also observed in InAs/GaAs Qdots material system [161, 162]. This is a property wherein remaining electrons from the surrounding material are supplied to the Qdots whose electroncs are extracted via stimulated emission triggered by the incident singal. Thus, the saturated gain (hole burning) in these Qdots which are resonant with the wavelength of the incident signal and the surrounding material which is unidque to Qdots helps in realizing an ultrafast gain recovery [154, 163]. Employing strong 150 fs resolution pump-probe experimentts, Zilkie et al. [164] measured a 15 ps long-lived and 1-2 ps short-lived gain recovery time in InAs/InGaAsP Qdots SOA on (100) InP and attributed, in general, to the phonon-mediated carrier capture in to Qdots, and slow $400 \mathrm{ps}$ absorption decay time due probably to the slow spontaneous carrier recombination, or carrier escape from the Qdots. Morevoer, comparison of Qdot, Qdash, and Qwell SOA behavior showed the gain recovery times to be fastest for dots (approximately 4-6 times smaller than Qwell) with the phase response following the same time scale as the gain recovery [165] indicative of small SOA linewidth enhanceent factor, and was confirmed by reporting values around 2-10 [166] with increasing current njection. In another study, Park et al. [167] performed similar pump-probe experiments to shed light on the contributions of GS and ES to the gain recovery time. It was shown, as depicted in Fig. 24(c) and (d), that for the GS wavelength, an ultrafast gain recovery time $\sim 0.7-2$ ps was extracted; however, at higher energy levels corresponding to the ES, the extracted gain recoevery time increased to $30 \mathrm{ps}$. Due to the peculiar nature, a three-step exponential fit was performed by Contestabile et al. [160] on the cross gain modulation signal obtained with a $0 \mathrm{~dB}$ CW signal at 1.55 $\mu \mathrm{m}$ and $3 \mathrm{dBm}$ mean power ( $13 \mathrm{dBm}$ peak power) for the $10 \mathrm{GHz}$ signal at $1.54 \mu \mathrm{m}$, to extract the gain recovery time. It was shown that around $85 \%$ of the gain recovery was achieved in only $10 \mathrm{ps}$ and complete gain saturation ( $100 \%$ recovery) in $\sim 30 \mathrm{ps}$.

\subsubsection{High speed amplification and signal processing}

The ability to achieve fast gain response to spectral hole burning, high output power and low noise, and the broad gain bandwidth of Qdots SOAs offered a variety of promising applications suh as aimplifiers, wavelength convertors, switches and regeneratores, in the optical network [168]. Here we review some of these achievments with regards to the InAs/InP Qdots SOA.

The high speed gain response to $10-40 \mathrm{GHz}$ signal intensity change was discussed in the previou section [154] with achieved gain of $>25 \mathrm{~dB}$ and $<5 \mathrm{~dB}$ NF. Similarly, the results of pumpprobe experiments in femtosecond resolution with gain recovery $\sim 2-15$ ps showed the potential of Qdots SOA for near $100 \mathrm{GHz}$ switching speed at $1.55 \mu \mathrm{m}$. The ability to operate with hih speed input signals (40 Gbps) with minimum signal distortion in the gain sauration regime (pattern effect) was demonstrated upto an output power of $22.8 \mathrm{dBm}$ (zero error free power penalty for up to 23.1 $\mathrm{dBm}$ output power) which is $8 \mathrm{~dB}$ superior compared to the Qwell SOA counterparts. Fig. 24(e) shows the bit error rate (BER) and eye diagrams of the Qdots SOA and Qwell SOA demonstrating 
superior preformance of Qdots device. In addition, Sugawara et al. [168] also demonstrated the Qfactor improvement of $40 \mathrm{Gbps}$ PRBS non-return-to-zero (NRZ) signal degraded by adding the amplified spontaneous emsission. The corresponding input and output signal eye diagrams were remarkably similar thus affirming Qdots SOA as a potenetial optical regenerator. The regenerating capability of the InAs/InP Qdots SOA was attributed to ' 1 '-level noise supression by driving the amplifier into gain saturation which could not be realized in Qwell SOA due to pattern effects. Furthermore, Contestabile et al. [169] reported utilization of Qdot SOA for regenerative amplification of 10, 20, and 40 Gbps return-to-zero (RZ) signals by a simple scheme exploiting self phase modulation with a detuned filter and working at low input power and with a large input dynamic range. The effect of high speed amplification of $10 \mathrm{Gbps}$ NRZ signal by Park et al. [170] revealed no singal distortion (pattern effects) at any applied current under GS operation of InAs/InP Qdots SOA, inline with the measured ultrafast gain recovery time. However, the slower gain recoevery componenet of the ES significantly deteorated the amplified output signal with patten effects when operated under ES.

InAs/InP Qdots SOA function as wavelength converstor was also reported recently utilizing two main non linear processes viz. FWM [171-173] and cross gain modulation [174]. In the former case, a symmetric FWM efficiency between positive and negative detuning was observed by Lu et al. [171], and the efficiency decreased by $<20 \mathrm{~dB} / \mathrm{decade}$ with frequency detuning increased. A 40 ps pulse train with repetition rate $500 \mathrm{MHz}$ was successufly detuned ut po $6.2 \mathrm{~nm}$ from the CW pump. By using low power single pump FWM, Contestabile et al. [172] reported a $30 \mathrm{~nm}$ conversion span with $>-10 \mathrm{~dB}$ conversion efficiency and output optical signal-to-noise ratio $>20 \mathrm{~dB}$. Transparency to coherent modulations was also demonstrated in a $25 \mathrm{~nm}$ conversion of 10 GBaud quadrature phase shift keying (QPSK), 8-phase shift keying (PSK) and 16-quadrature amplitude modulation (QAM) signals. Very recently, utilizing double pump FWM process, an ultra-broadband spanning $100 \mathrm{~nm}$ and efficient wavelength conversion (positive conversion efficiency $>30 \mathrm{~dB}$ output optical signal-to-noise-ratio) was reported in the telecommunication band around $1.55 \mu \mathrm{m}$ [173]. In the latter case of cross gain modulation, employing InAs/In GaAsP Qdot SOA on (100) InP, an efficient distortion free wavelength conversion on detuning of $\sim 12 \mathrm{~nm}$ was achieved for frequencies upto $40 \mathrm{Gbps}$ [174]. For frequencies 80 and $160 \mathrm{Gbps}$, a slightly detuned ou tput filter was utilized to obtain pattern-effects free wavelength conversion via cross-phase-modulation. Moreover, with a 80 Gbps signal format conversion of output inverse-RZ to RZ was also demonstrated.
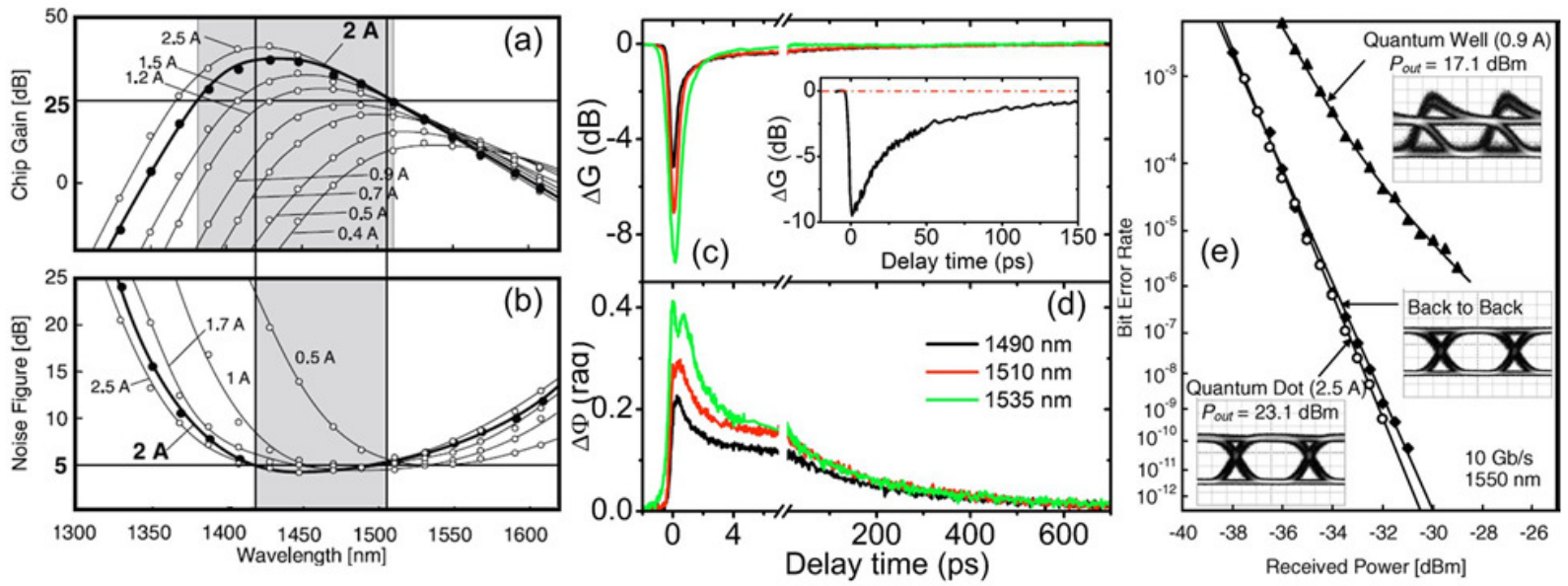

Fig. 24. Wavelength dependence of (a) gain and (b) NF, as a function of wavelength for the InAs/InGaAsP Qdots SOA. Pump and probe measurements of the (c) gain and (d) phase recovery curves at $1.535 \mu \mathrm{m}(\mathrm{GS})$ and 1.510 and $1.490 \mu \mathrm{m}(\mathrm{ES})$ under an applied current of $300 \mathrm{~mA}$ for the InAs/InP Qdots SOA. Inset shows the gain recovery curve of a commercial SOA composed of a bulk active material. (e) B it error rate (BER) and eye diagrams of the InAs/InP Qdots SOA. Reproduced from [167, 168].

\subsection{InAs/InP Qdots superluminescent diodes}


Long wavelength SLD working in the C-band are highly attractive in optical measurement and instrumentation, OCT, etc., besides optical communications, as has been discussed in section 1. In the case of In As/InP Qdots based active region, by exploiting the inhomogeneous broadening via modulated stacking of the self-assembled InAs Qdots on strain reducing InGaAlAs layer on (311)B InP substrate, Akahane et al. [175] reported a room temperature ultra-broadband SLD operating under pulsed operation. By varying the amount of InAs deposition among the Qdots stacks and the thickness of the buffer/spacer layer (chirped design), a 36-stack device structure was grown using MBE with Qdot density $7.1 \times 10^{10} \mathrm{~cm}^{-2}$. The $50 \mu \mathrm{m}$ wide device under pulsed operation demonstrated an electrolumenescence bandwidth of $213 \mathrm{~nm}$ at $100 \mathrm{~mA}$ centered at around $1.53 \mu \mathrm{m}$. A maximum bandiwdth of $217 \mathrm{~nm}$ was achieved at $3 \mathrm{~A}$, and the measured coherence length using Michelson interferrometer was $6.5 \mu \mathrm{m}$ which is impressive and probably the best value reported in literature around this wavelength. 


\section{InAs Qdashes grown on InP}

The word "quantum dash" was introduced in late 1990"s by Utzemier et al. [176] and Guo et al. [177] who reported self-assembled InSb Qdashes on (100) InP and In As Qdashes on (211)B GaAs substrates, respectively. The Qdashes were elongated along [110] direction in the former case and [110] direction in the latter case with typical dimensions $\sim 18 \mathrm{~nm} \times 60 \mathrm{~nm} \times 140 \mathrm{~nm}$. It was until early 2000's that this class of quantum confined nanostructure key features were identified and exploited in the demonstration of various optoelectronic devices. In this section, we reviewed the Qdash growth on (100) InP substrate starting from the self-assembled quantum-wire (Qwire) growth because this work laid the foundation for eventual Qdash growth technology. This nanostructure was also dominated by two material system (i) InAs/nGaAlAs and (ii) InAs/InGaAsP, similar to that of the InAs/InP Qdot case discussed in section 2 and 3.
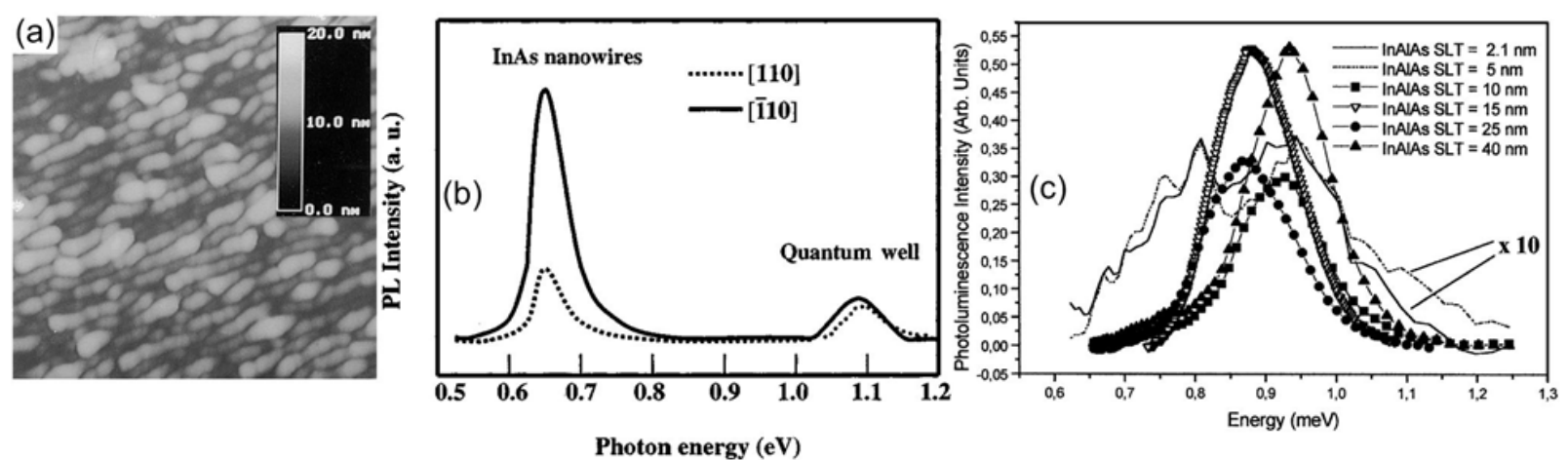

Fig. 25. (a) AFM image of $\left(1 \times 1 \mu \mathrm{m}^{2}\right)$ InAs surface morphology with 7.5 ML deposition thickness on $\mathrm{A} b_{0.48} \mathrm{In}_{0.52} \mathrm{As}$ showing the growth anis otropy. (b) $15 \mathrm{~K}$ polarized PL spectra of the multilayer sample with 6 stack InAs $\sim 6.5 \mathrm{ML} / \mathrm{A} b_{6.48} \mathrm{In}_{0.52} \mathrm{As} \sim 20 \mathrm{~nm}$. The incident laser beam was polarized in the [110] or [-110] direction. (c) Comparison of $300 \mathrm{~K}$ PL spectra of 5-stack InAs layer samples with different spacer layer thickness (SLT). Collected from $[28,178,179]$.

\subsection{InAs/InGaAlAs Material System}

\subsubsection{Qdashes on (100) InP substrate}

Research interest in the MBE growth of self-organized InAs Qwires or wire-like nanostructures on (100) InP substrate started in late 1990's with in itial demonstrations by Brault et al. [23] and Li et al. [28] on In AlAs buffer layers. In the latter case, high density wire-like nanostructures, shown in Fig. 25 (a), were observed from the 6-stack InAs/Al ${ }_{0.48} \mathrm{In}_{0.52} \mathrm{As}$ Qdashes $(\sim 70 / \mu \mathrm{m})$ and ascribed to the surface an isotropy due to As-dimer reconstruction. Moreover, linearly polarized light based $15 \mathrm{~K} \mathrm{PL}$ measurements revealed peak PL wavelength at $\sim 1.9 \mu \mathrm{m}$ and the wires exhibited large anisotropy for [1-10] and [110] polarization with peak PL intensity ratio of 3, as depicted in Fig. 25(b). Subsequently, Li et al. found via TEM characterization on a single stack InAs/ $\mathrm{Al}_{0.48} \mathrm{In}_{0.52} \mathrm{As}$ Qwire structure that increasing the amount of InAs deposition from 1.5-7.5 ML resulted in more inhomogeneous and large size Qwire formation [178]. This work was soon followed by a thorough investigation of the effect of AlInAs spacer/buffer layer thickness in a multi-stack Qwire structure on its optical characteristics by Brault et al. [179]. A fixed 5-stack Qwire sample with 3 ML InAs deposition thickness and $120 \mathrm{~s}$ growth interruption was employed in the study, and varying the AlInAs thickness from $5 \mathrm{~nm}$ to $40 \mathrm{~nm}$. For $<10 \mathrm{~nm}$ spacer layer thickness (SLT), a highly dispersive Qwire formation with no vertical self-organization of the Qwire layers was observed and verified by large room temperature PL linewidth of $\sim 300 \mathrm{meV}$ centered at $\sim 1.77 \mu \mathrm{m}$, as illustrated in Fig. 25(c). A strong staggered Qwire vertical arrangement was found for 10-25 nm SLT with increase in the Qwire size and reduction in PL linewid th to $\sim 130 \mathrm{meV}$, while no correlation was observed between the SLT and Qwire formation beyond $25 \mathrm{~nm}$ which suggested no influence of dash layer on each 
other. These observations were attributed to the complex interdependence between phase separation, surface morphology, and mismatched stresses [179] within the structure.

Next, Wang et al. [3] reported the first dash-in-a-well (DaWELL) laser structure on (100) InP substrate by growing InAs Qdashes within an asymmetric compressively strained InGaAlAs Qwell separated by In GaAlAs tensile strained barrier layers. A Qdash density $\sim 10^{10} \mathrm{~cm}^{-2}$ with typical Qdash dimensions of $\sim 5 \mathrm{~nm} \times 25 \mathrm{~nm} \times 300 \mathrm{~nm}$ was reported, via AFM study. Room temperature PL peak emission was observed at $\sim 1.57 \mu \mathrm{m}$ and linewidth $\sim 75 \mathrm{meV}(\sim 140 \mathrm{~nm})$. Following this work, Schwertberger et al. [180] performed a systematic study of different MBE growth parameters influencing InAs Qdash formation on InGaAlAs buffer layer on (100) InP substrate. By fixing the substrate temperature and increasing the In As deposition thickness from $2.5 \mathrm{ML}$ to $7 \mathrm{ML}$ drastically affected the Qdash morphology with decreasing Qdash length and increasing Qdash height. For instance, SEM image of 5 ML InAs deposition thickness Qdashes is shown in Fig. 26(a). On further increase in InAs thickness, single Qdashes coalesce to form larger islands of the material with reduced Qdash like nature. This study was further supported by PL results at $8 \mathrm{~K}$ from these samples as depicted in Fig. 26(b). A clear red shift in the PL peak emission from $1.2 \mu \mathrm{m}(0.4 \mathrm{~nm})$ to $2.0 \mu \mathrm{m}$ $(10 \mathrm{ML}, 3.1 \mathrm{~nm})$ was observed on increasing the InAs deposition thickness [181]. Furthermore, the effect of substrate temperature on the Qdash growth was examined Schwertberger et al. and suggested an ideal growth temperature around $500^{\circ} \mathrm{C}$ which showed maximum PL intensity without any change in the PL peak position. Any temperatures higher than $500^{\circ} \mathrm{C}$ was not realized due to increased In desorption during Qdash growth. In another study, Stintz et al. [38] also reported similar results on the effect of InAs coverage (8-23 $\AA$ ) on the Qwire formation on InGaAlAs and InGaAs buffer layers ascribing the asymmetric nanostructure formation to the anisotropy of strain relaxation, difference in surface mobility and diffusion length with direction. However, beyond these InAs coverage the directional dash preference was observed to decrease with almost no evidence of dotlike structures at $23 \AA$ In As deposition, and the PL measurements suggested large misfit dislocations in these Qdots as no light emission was observed. Furthermore, by room temperature PL measurements on DaWELL samples with Qdashes embedded in InGaAlAs and InGaAs Qwell, surrounded by InGaAlAs barrier layers, Rotter et al. showed a huge red shift with respective PL peak emission at $1.52 \mu \mathrm{m}$ to $2.04 \mu \mathrm{m}$; a notable demonstration of the tunability of Qdash emission by the surrounding Qwell layers and InAs coverage [182].
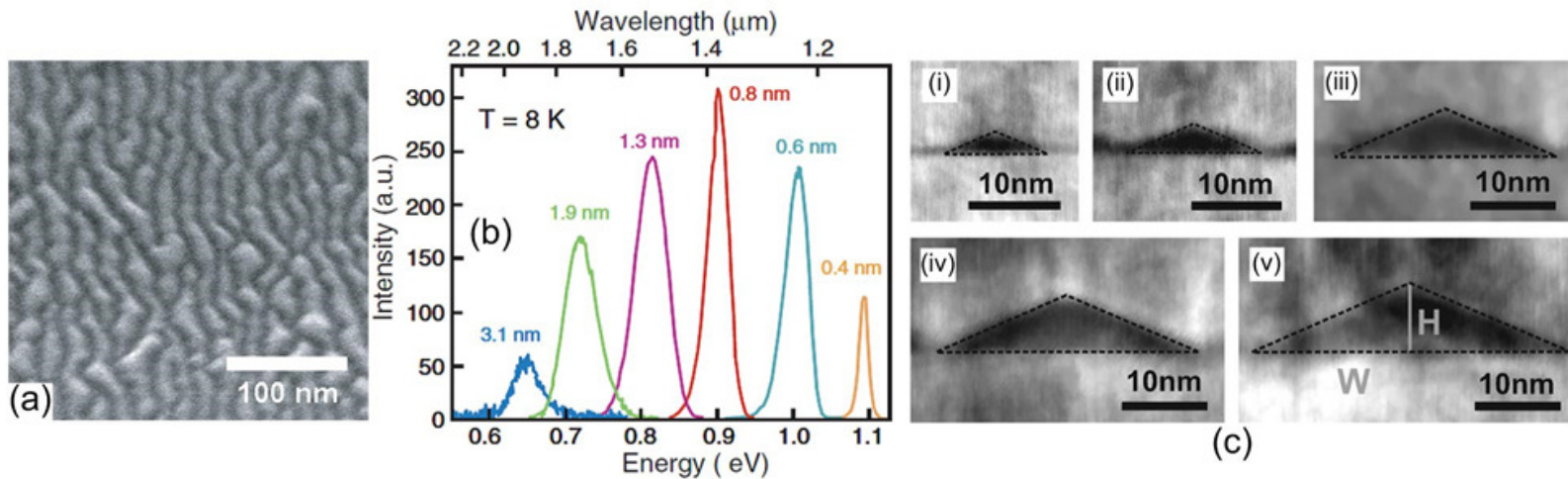

Fig. 26. (a) SEM image of the top view of an uncapped InAs Qdash sample with 5.0 ML InAs grown on InGaAlAs buffer layer on (100) InP substrate. (b) L ow temperature PL spectra of Qdash layers with different nominal InAs layer thickness as indicated in the figure, on InGaAlAs buffer layer on (100) InP substrate. (c) Cross-sectional bright-field TEM micrographs of the InAs Qdash samples with InAs nominal thicknesses of 0.8, 1.2, 1.6, 2.4, and $3.1 \mathrm{~nm}$ for samples (i), (ii), (iii), (iv), and (v), respectively, on InGaAlAs buffer layer. The parameters ' $\mathrm{H}$ ' and ' $\mathrm{W}$ ' in (v) correspond to the Qdash height and width. Adapted from [26, 183].

Next, Sauerwald et al. [183] demonstrated, via chemically sensitive scanning TEM and PL measurements, that by adjusting a single growth parameter i.e. InAs deposition thickness, the Qdash emission can be controlled between 1.37 and $1.9 \mu \mathrm{m}$ without modifying its shape and composition. By MBE growth of single stack InAs/In GaAlAs Qdash samples on (100) InP substrate at various In As coverage $(0.8,1.2,1.6,2.4$, and $3.1 \mathrm{~nm})$, they showed that Qdashes maintained a constant height-to-width ratio within the sample and among the samples, as shown in Fig. 26(c). Moreover, 
the Qdash height and width increased linearly (i.e. Qdash size) with increasing nominal InAs layer thickness, thus red shifting the PL peak wavelength and broadening the PL linewidth. No significant intermixing of Qdash and the embedding material was observed, and the PL shift was attributed to the change in Qdash size (smaller dashes with higher energy quantization shifted to higher energies) [184]. In addition to the growth kinetics, surface and strain energies also played an important role in the Qdash formation and hence this observation was also related to the significant reordering of the material during the whole growth of the InAs layer. This work of single stack Qdash was further extended to a multi-stack Qdash structure with the objective of examining the impact of vertical stacking on the size and composition of Qdashes [185]. By growing 4-stack InAs/InGaAlAs DaWELL structure in three samples, each with different InAs layer thickness $(1.1 \mathrm{~nm}, 1.5 \mathrm{~nm}$, and $2.1 \mathrm{~nm})$, it was shown that the Qdash size increased with subsequent stacking of layers. This effect was found to increase with nominal InAs layer thickness and was ascribed to the strain effects that caused the merging of Qdashes in the upper layers and hence increase in size leading to larger inhomogeneity in the DaWELL structure.
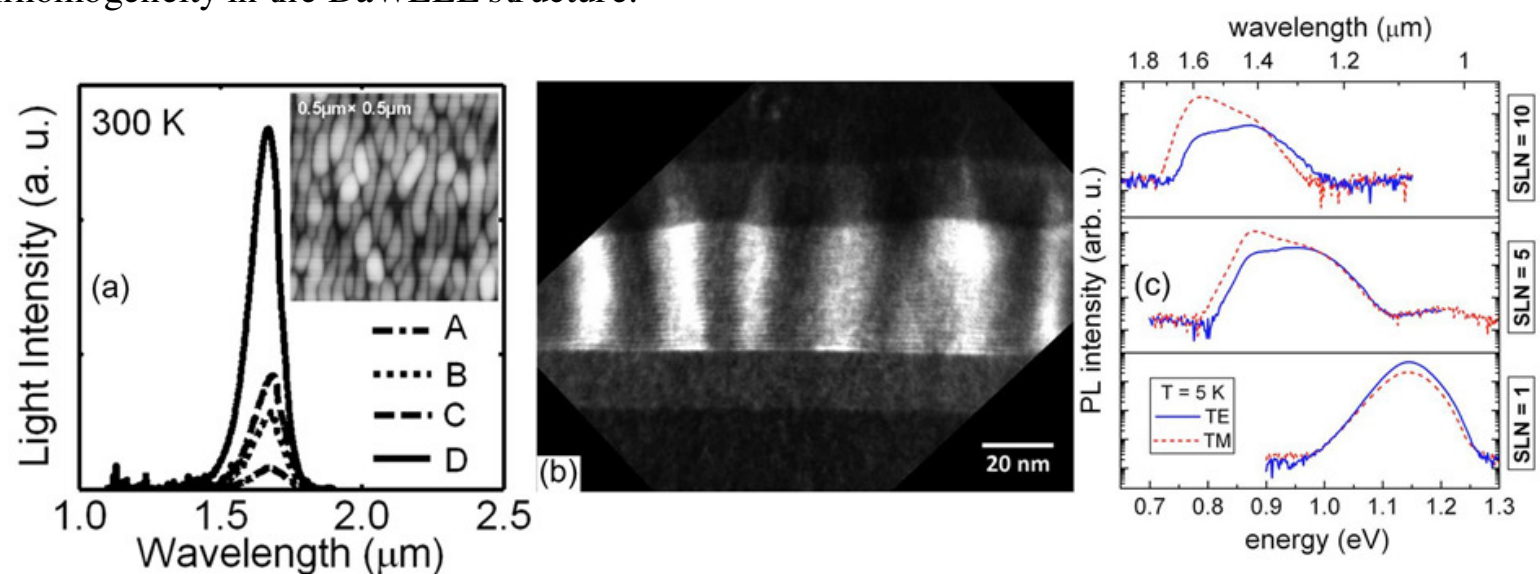

Fig. 27. (a) PL spectra measured at $300 \mathrm{~K}$ for four InAs Qdash samples grown at various conditions (A and B with InGaAlAs barrier grown at $505{ }^{\circ} \mathrm{C}$ and $535^{\circ} \mathrm{C}$, respectively. $\mathrm{C}$ and D are similar to B except an ultra-thin GaAs layer was grown before the growth of InAs layer, and before and after the growth of InAs layer, respectively). Inset shows the $\left(0.5 \times 0.5 \mu \mathrm{m}^{2}\right) \mathrm{AFM}$ image of an InAs Qdash layer without InAlGaAs cap. (b) Cross-sectional TEM micrograph of InAs/GaAs columnar Qdashes with 24 stacking layers. The intersection was made in [110] direction, i.e., perpendicular to the longer lateral size of the Qdashes. (c) Polarization resolved low temperature PL from the cleaved edge perpendic ular to the columnar Qdashes' longer lateral size. Stacking layer number (SLN) is equal 1, 5, and 10, where SLN=1 corresponds to the structure with standard Qdashes. Courtesy of [186, 187].

In order to improve the inhomogeneous broadening of Qdash multi-stack structure, the concept of growing ultrath in ( 2 ML) GaAs layer on InGaAlAs barrier layer before InAs deposition for the Qdash formation was reported by Mi et al. [186] who demonstrated an improvement in the optical quality ( $>3$ times increase in the PL intensity) as illustrated in Fig. 27(a) and is coherent with other reports [36]. A dramatic enhancement (> 10 times) in PL intensity with reduced PL linewidth of $\sim 50 \mathrm{meV}$ at room temperature was observed if additional GaAs layers were deposited after the growth of $5 \mathrm{~nm}$ In GaAlAs capping layer. This was attributed to the suppressed phase separation and enhanced surface migration in the barrier layer. A PL peak wavelength of $\sim 1.7 \mu \mathrm{m}$ and Qdash density of $\sim 2 \times 10^{10} \mathrm{~cm}^{-2}$ was reported. Furthermore, modulation p-doping the active region (barriers) and tunnel injection schemes were also investigated on the multi-stack Qdash structures without affecting the Qdash quality [188]. Very recently, Podemski et al. [187] reported the growth of highly stacked columnar Qdashes on (100) InP. The nominal thickness of the Qdash layers were $\sim 0.7 \mathrm{~nm}$ (just above the critical thickness of Qdash formation) with $\sim 1.0 \mathrm{~nm} \mathrm{GaAs} \mathrm{spacer} \mathrm{layer,} \mathrm{and} \mathrm{hence}$ formation of thin dashes, as shown in TEM image of Fig. 27(b). The Qdash layers were then sandwiched between InGaAlAs layers and finally with InAlAs buffer layers. It was shown that by controlling the number of stacking layers (SLN) from 1 to 24 , a transition of the dominant polarization from transverse electric to transverse magnetic could be realized which is highly attractive for polarization independent SOA. Besides, plotted in Fig. 27(c), a red shift in the PL peak emission was observed on increasing the number of Qdash layers (from $\sim 1.1 \mu \mathrm{m}$ to $\sim 1.6 \mu \mathrm{m}$ ). In general, these findings were attributed to the increase in Qdash vertical dimensions when PL 
intensity from the cleaved facet edge perpendicular to the columnar dashes longer lateral size was acquired. However, emission from the other edge (parallel to the Qdashes' longer lateral size) did not show any dependence of polarization on the dash height since the polarization was driven by the dashes longer lateral size rather than height [189]. A more detailed and systematic investigation of the Qdash height on the polarization of the surface emission was carried out by Musial et al. [190] who showed through surface PL emission from these dashes was primarily driven by Qdash height due to heavy hole and light hole mixing.

\subsubsection{Qdash Optical Properties}

Ukhanov et al. observed anisotropy in modal gain which was due to the polarization dependence of the transition matrix element in the Qdash nanostructures [191]. The experimental measurements of gain, differential gain, and LEF showed a strong Qdashes-orientation dependence in the laser cavity. Qdashes parallel to the cavity showed a peak gain blue shift of $10 \mathrm{~nm}$ compared to the Qdashes perpendicular to the cavity and peak gain difference of $>5 \mathrm{~cm}^{-1}$ below the threshold. This was attributed to the addition of light-hole contributions in to the primarily heavy-hole transitions in these Qdashes. This study was further supported by Hein et al. [189] and Podemski et al. [187] via columnar Qdashes growth, stating a strong relationship between the polarization degree of emission and the orientation of the columnar Qdashes. Besides the above findings, Popescu et al. [192] showed that geometrical asymmetry of Qdashes influences the carrier migration (ambipolar carrier migration length), and noticed a $20 \%$ reduction in carrier migration along Qdash elongation compared to that across the dash elongation (i.e. perpendicular to elongation).

Further detailed investigations on the optical properties of this new class of nanostructures grown on InAs/InGaAlAs/(100)-InP platform were performed. For instance, thermal quenching of the PL was studied by temperature dependent PL on In As/InGaAlAs Qdash structures with deduced two activation energies which were ascribed to electron escape and heavy hole escape to the conduction and valance band in Qdash barriers, respectively [193]. A similar temperature dependent carrier dynamics was also carried out by Jahan et al. [194] on InAs/InAlAs Qdash heterostructure. Using PL and photo-reflectance (PR) measurements on a single Qdash layer embedded in InGaAlAs buffer layers, Rudno-Rudzinski et al. showed the existence of $\sim 2$ ML thick wetting layer [195], observed the presence of ES at an energy of $~ 150 \mathrm{meV}$ above the GS transition in Qdashes [196], and studied the interband optical transitions in DaWELL structure with InGaAs/InGaAlAs Qwells [197]. In addition, Podemski et al. investigated the efficiency of excitons and free carrier injections in Qdash tunnel-injection structures via PL measurements [198] and measured Qwell-Qdash energy transfer up to $130 \mathrm{~K}$ employing PL excitation (PLE) study [199]. Marko et al. [200] showed in the high pressure measurements on Qdash-lasers that Auger recombination in Qdashes was the major contributor to the high threshold current density at room temperature.

\subsection{InAs/InGaAsP Material System}

\subsubsection{Qdashes on (100) InP substrate}

Early work of Qdash growth on this material system started from the investigations on InP buffer layer on nominal [22] and vicinal [201, 202] (100) InP substrates on MBE system. Gonzalez et al. [22] demonstrated that the buffer layer surface morphology played an important role in the formation of InAs nanostructures by growing the InP buffer layer using atomic-layer MBE and MBE. Under identical growth condition, the former growth method resulted in self-assembled Qwire-like nanostructures elongated along [1-10] direction while the latter outcome was Qdot-like formation. Later, via in-situ stress measurements, it was elucidated that the associated anisotropic stress relaxation of the heteroepitaxial system involving different group V elements was the key cause for Qwire formation [203]. On the other hand, Walther et al. [201] showed Qwire growth on both exact and vicinal [2 $2^{\circ}$ offcut along (-110)] (100) InP substrates employing InP, InGaAs and AlInAs buffer layers, and growth interruption after InAs deposition. This was attributed primarily 
due to strain and discarded the contribution due to the chemical details of the buffer layer under the given growth parameters. An AFM image of Qwires on AlInAs buffer layer is shown in Fig. 28(a). This work was further supported by Salem et al. [202] who also compared the nanostructure growth on nominal and $2^{\circ}$ offcut [along (-110) and (010)] (100) InP substrates and found Qwire-like formation on the former two substrates and Qdot on the latter substrate. A low temperature $(8 \mathrm{~K}) \mathrm{PL}$ peak emission at $\sim 1.46 \mu \mathrm{m}$ with linewidth $\sim 75 \mathrm{meV}$ was observed from these Qwire nanostructures.
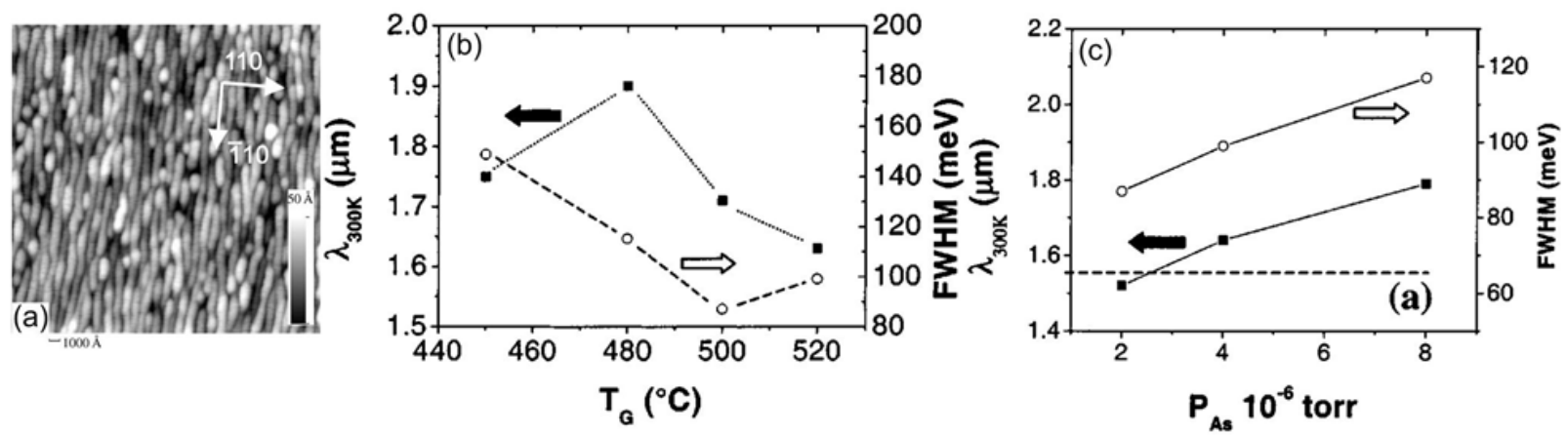

Fig. 28. (a) AFM image of InAs wires (InAs deposition thickness $~ 1.2 \mathrm{~nm}$ ) grown on a ternary $\mathrm{A} b_{.52} \mathrm{In}_{0.48} \mathrm{As}$ buffer layer on (100) InP (b) PL characteristics [peak emission and linew idth] of InAs island PL spectra on InP buffer layer at $300 \mathrm{~K}$ as a function of the growth temperature for an InAs de position thickness just above $(\sim 0.5 \mathrm{ML})$ the critical thickness. (c) PL characteristics of InAs islands on InP at $300 \mathrm{~K}$ versus the As overpressure during the InAs growth (growth temperature $520^{\circ} \mathrm{C}$, InAs deposition thickness $0.9 \mathrm{~nm}$ ). Taken from [201, 204].

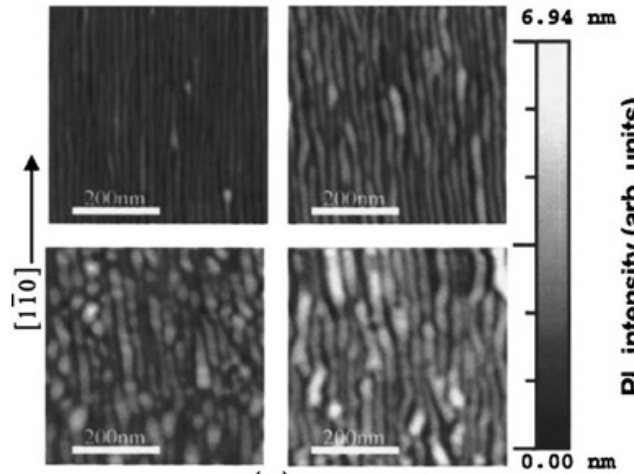

(a)
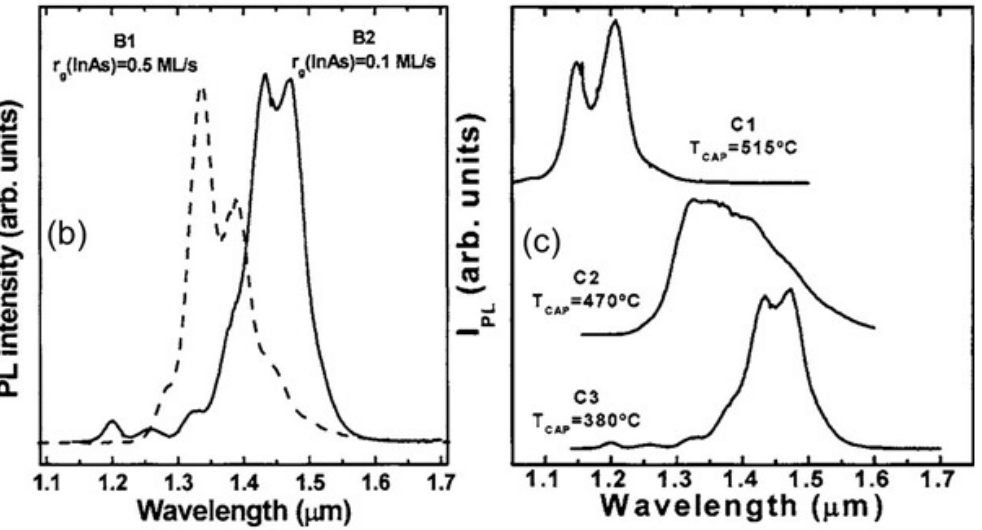

Fig. 29. (a) AFM images $\left(0.5 \times 0.5 \mu \mathrm{m}^{2}\right)$ of four uncapped InAs Qw ire samples with different InAs de posited thicknesses $2.5 \mathrm{ML}, 3.3 \mathrm{ML}, 4.3 \mathrm{ML}$, and 5.3 ML from upper left to bottom right. The four images have the same z-scale bar. (b) $12 \mathrm{~K}$ PL spectra of InAs Qwires grown at different InAs growth rates of 0.1 and $0.5 \mathrm{ML} / \mathrm{s}$. (c) $12 \mathrm{~K} \mathrm{PL}$ spectra of InAs Qwire samples where the InP cap layer has been grown at different substrate temperature s $T_{\mathrm{CAP}}=$ 380,470 , and $515^{\circ} \mathrm{C}$. Collected from [205].

Later, Gendry et al. [204] addressed the size dispersion and wavelength tunability issue of In As quantum-stick nanostructures. They showed employing a single stack of quantum-sticks in InP matrix layer that optimized epitaxial growth parameters could result in reduced nanostructure height dispersion. First, by increasing the growth temperature which in turn lowers the critical thickness for 2D/3D (three-dimensional) growth mode transition, they observed uniform islands growth, as depicted in Fig. 28(b). The measured room PL linewidth of the sample grown at $520^{\circ} \mathrm{C}$ (InAs deposition $0.5-0.65 \mathrm{~nm}$, just above the critical thickness at this temperature) was $\sim 100 \mathrm{meV}$ compared to $\sim 170 \mathrm{meV}$ for the sample grown at $480^{\circ} \mathrm{C}$ (InAs deposition $1.6-1.8 \mathrm{~nm}$, just above the critical thickness at this temperature). In addition, the tuning capability of the islands emission energy via InAs layer thickness variation was also highlighted; with the former sample PL peak wavelength at $\sim 1.64 \mu \mathrm{m}$ compared to the latter sample at $\sim 1.9 \mu \mathrm{m}$. In brief, reducing the As overpressure during InAs deposition (see Fig. 28(c)), increasing the InAs thickness to a much larger values than the critical thickness for the 2D/3D growth mode transition, and higher growth temperature, were shown to be the optimum conditions for low height dispersion grown selforganized quantum-sticks. In fact, a PL linewidth of $\sim 50 \mathrm{meV}$ was reported at $8 \mathrm{~K}$ from the 
optimized single stack structure with island density $6 \times 10^{10} \mathrm{~cm}^{-2}$. This achievement was accredited to the kinetic and thermodynamic factors such as adatom surface diffusion, strain accumulation, etc., that benefited the island self-organization [204].

In continuation to the growth parameter optimization for quantum islands size and emission control, Fuster et al. [205] studied the effect of InAs layer thickness on the Qwire formation and found that large Qwire formed with increased InAs layer thickness, as depicted in Fig. 29(a), which was consistent with other reports on the InAs/In GaAlAs material system, reviewed in section 4.1. Next, they explored two additional methods that depends on the As/P exchange process control for Qwire formation; firstly, during InAs growth of Qwires, and secondly, during the InP capping layer deposition to cover the nanostructures. An increase in the InAs deposition rate from $0.1 \mathrm{ML} / \mathrm{s}$ to 0.5 ML/s (keeping other growth parameters fixed) resulted in PL emission blue shift of $\sim 100 \mathrm{~nm}(\sim 1.45$ $\mu \mathrm{m}$ to $\sim 1.35 \mu \mathrm{m}$ ) deduced from the 12K PL experiment, as shown in Fig. 29(b). This was attributed to the exchange between As and $\mathrm{P}$ atoms at the InP surface exposed to As flux during InAs growth. In general As/P exchange process produces source of In at the expense of the InP buffer layer quality, resulting in an effective InAs growth rate higher than the deposition rate, and hence larger size wires with higher heights (emitting at longer wavelengths) were grown at slow InAs growth rates. On the other hand, the PL spectrum shifted to shorter wavelengths as the substrate temperature during capping layer growth was increased from $380^{\circ} \mathrm{C}$ to $515^{\circ} \mathrm{C}$ as illustrated in Fig. 29(c). In this case, P/As exchange at the interface between the InAs wires and InP capping layer when exposed to $P$ flux during InP capping layer growth affected the height of the InAs Qwires [206].
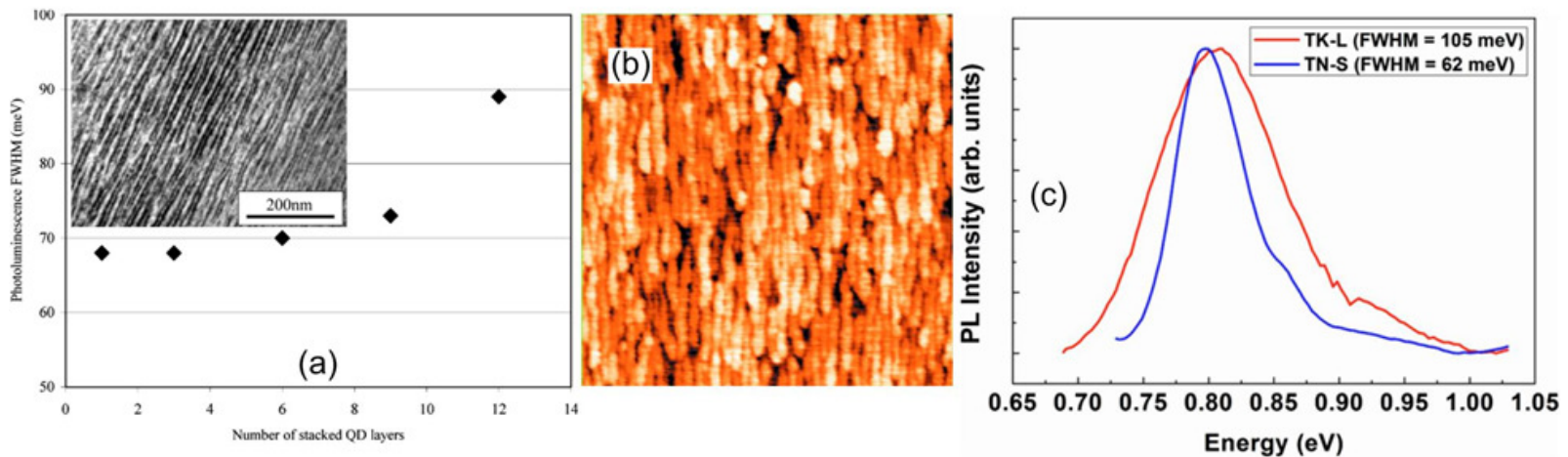

Fig. 30. (a) Room temperature PL linewidth of stacked InAs/InGaAsP Qdashes as a function of the number of stacking layers. Inset shows the planview TEM micrograph of a stack of 6 Qdash layers. (b) AFM image $\left(1 \times 1 \mu \mathrm{m}^{2}\right)$ of an uncapped 3 stacked InAs/InGaAsP Qdash sample, and (c) room temperature PL spectra of a 5 stack Q dash sample with a thicker InGaAsP first capping layer and a longer growth interruption time (TK-L) and a Qdash sample with a thinner InGaAsP first capping layer and a shorter growth interruption time (TN-S) sample. Courtesy of [6, 7, 207].

Subsequently, growth and optical characterization of multi-stack Qwire structures were reported by Alen et al. [208] where 10 stacks of InAs/InP Qwires were grown with $5 \mathrm{~nm}$ and $10 \mathrm{~nm}$ spacer thickness for comparison. It was shown that smaller spacer layer thickness structure resulted in Qwire size homogeneity and uniformity of Qwire stacks attributed to the strain driven vertical filtering of the Qwire sizes along the growth direction. Low temperature PL peak emission around $1.54 \mu \mathrm{m}$ was observed with $\sim 100 \mathrm{meV}$ PL linewidth. In contrast, the thick InP spacer layer structure was highly inhomogeneous with broad PL linewidth of $\sim 350 \mathrm{meV}$ due to inhibition of strain propagation and hence no size filtering (vertically uncorrelated Qwires). The results of this work is in line with Podemski et al. [187] work on InAs/InGaAlAs material system. Following this work, a detailed analysis on the effect of capping layer thickness on 2-stack and 5-stack Qwire structures inhomogeneity was carried out by Fuster et al. [209] utilizing the in-situ and real time stress measurements and reflection high-energy electron diffraction (RHEED) observations besides relating to the P/As switching process. Rather than reducing the barrier layer thickness for achieving homogeneous size Qdashes, Lelarge et al. [207] recently developed a solution to achieve the same with a barrier thickness of $40 \mathrm{~nm}$. They utilized an empirical rule involving the growth conditions, number of Qdash layers, thickness, and the strain of barriers as key parameters to identify the nominal thickness of the subsequent dash layer in order to compensate for the natural thickening of 
the Qdashes during stacking. With this method MBE grown Qdashes up to 10 stacks were achieved with out affecting the room temperature PL linewidth (maintained at $\sim 70 \mathrm{meV}$ ), as illustrated in Fig. 30(a). The optimized double capping technique, primarily developed for the InAs/InP Qdots growth (discussed in section 2.2), was also employed by Zhou et al. [6, 7] to obtain homogeneous Qdashes and to tune the emission wavelength of a single and multi-stack InAs/InGaAsP Qdash structures on (100)InP substrate. By selecting a thin $2.2 \mathrm{~nm}$ first capping layer and short $30 \mathrm{~s}$ growth interruption time, they measured a room temperature PL linewidth of $60 \mathrm{meV}$ from 5 stacked Qdash structure (TN-S) centered at $1.55 \mu \mathrm{m}$, shown in Figs. 30(b) and (c).

Very recently, Lenz et al. [210] explored the formation of Qdashes on In GaAsP buffer layers on (100) InP grown by MOCVD system. 2.65 ML thick InAs deposition thickness was utilized for Qdash growth in a single and 7 stack structure, separated by $40 \mathrm{~nm}$ InGaAsP barrier layers. A series of samples at different growth temperature $\left(470^{\circ} \mathrm{C}\right.$ to $\left.530^{\circ} \mathrm{C}\right)$ and TMI flow rate $(13$ to $200 \mathrm{sccm})$ revealed Qdash formation at low temperature $\left(\leq 500^{\circ} \mathrm{C}\right)$ and/or high flow rate $(\geq 100 \mathrm{sccm})$. The PL linewidth of $\sim 60 \mathrm{meV}$ centered at $\sim 1.55 \mu \mathrm{m}$ was reported at room temperature. Moreover, composition fluctuation of the InGaAsP material towards the InAs rich and GaP rich regions was also noted. Alternatively, Faugeron et al. [211] presented the concept of an asymmetrical cladding in the Qdash active region. An undoped intermediate optical index slab layer was inserted between the Qdash active zone and the substrate in the 6-stack Qdash-in-barrier structure with the aim of reducing the overlap of the optical mode with the lossy p-doped cladding layers. The room temperature PL peak wavelength was shown to be independent of the slab layer thickness, at $\sim 1.57$ $\mu \mathrm{m}$ with no change in the PL linewidth, thus maintaining the optical quality of the material.

\subsubsection{Qdash Optical Properties}

The radiative optical transitions available in InAs/InP self-assembled Qwire structure was investigated by Alen et al. [212] employing PL and absorption measurements. Several optical transitions were observed at $85 \mathrm{~K}$, consistent with both the measurements, and were attributed to Qwires of different height. In addition, the non-radiative recombination mechanism responsible for the quenching of PL was related to the thermal carrier escape of carriers out of the wires towards the barrier material. In contrast, Salem et al. [213] reported that the multicomponent transitions from the InAs/InP quantum islands structure showed the existence of excited states and substantiated the results with PLE measurements at $8 \mathrm{~K}$. In another study, Heck et al. [214] studied the band structure of the DaWELL and Qdash-in-barrier InAs/InGaAsP structures on (100) InP and found that electron states are not confined in the former structure while weakly confined in the latter one due to small effective mass and conduction band offset [215].

\subsection{Post-growth tuning of InAs/InP Qdashes}

Our group pioneered in the intermixing study of InAs/InGaAlAs DaWELL nanostructures with the first demonstration of $e x$-situ defect annealing and wavelength tuning using the IFVD process with $200 \mathrm{~nm} \mathrm{SiO} 2$ capping by Djie et al. [216]. As shown in Figs. 31(a) and (b), a linear increase in the low temperature (77 K) PL peak blue-shift was observed in a 4 stack Qdash structure with increasing annealing temperature, attributed to the strong thermally induced group III intermixing effects and reaching a maximum value of $180 \mathrm{~nm}$ at $850^{\circ} \mathrm{C}$. In addition, a strong increase in the radiative efficiency indicated by the improvement in the integrated PL signal (30-40\% increase) was reported at $700^{\circ} \mathrm{C}$ compared to the as-grown Qdash structure, with minimal PL peak shift $(\sim 1.532 \mu \mathrm{m}$ versus $\sim 1.54 \mu \mathrm{m}$ at low excitation power). This improvement in the material quality after intermixing was related to the reduced defect density which might be presented near the DaWELL interfaces due to low growth temperature and increased stress field. A subsequent device fabrication and improved performance was also demonstrated which will be discussed in section 5.1. On the other hand, it was shown that intermixing was enhanced under a $\mathrm{Si}_{\mathrm{x}} \mathrm{N}_{\mathrm{y}}$ cap at the same temperature of $700^{\circ} \mathrm{C}$ with a differential PL peak blue shift of $80 \mathrm{~nm}$ (PL peak at $\sim 1.46 \mu \mathrm{m}$ at low excitation power), as shown in 
Fig. 31(c), and was attributed to the different group-III intermixing rates [217]. In addition, the material quality was maintained with no significant broadening of the PL linewidth.

A spatial control of group-III intermixing rate by IID technique employing nitrogen ions (NIID) was also reported on four stacks InAs/InGaAlAs DWELL structure separated by $30 \mathrm{~nm}$ tensile strained InGaAlAs barrier layer [218], and plotted in Fig. 31(d). At low temperature annealing of $650^{\circ} \mathrm{C}$ and $700^{\circ} \mathrm{C}$ a large blue shift in the PL peak of the samples implanted with $5 \times 10^{12}$ and $5 \times 10^{13}$ ions $/ \mathrm{cm}^{2}$ was observed with a value $\sim 112 \mathrm{~nm}$ compared to the bare as-annealed samples. The PL blue shift value reduced to $\sim 108 \mathrm{~nm}$ at $5 \times 10^{14}$ ions $/ \mathrm{cm}^{2}$ ascribed to the formation of clusters that trapped point defects during annealing treatment and effective reduction in defect concentration. An indirect NIID (implantation to the $300 \mathrm{~nm}$ thick $\mathrm{SiO}_{2}$ protected sample) process was also carried out at various ion doses to assess the placement of implantation induced damage into the sample and correlate the defect migration and intermixing efficiency. An integrated PL intensity comparable to the as-grown sample was observed with indirect NIID process for all the above implantation doses while the direct NIID showed weaker PL intensity, thus validating the fact that residual defects generated post-annealing with indirect NIID process was minimum. The optical quality of the active region was retained during the process. The PL peak wavelength and blue shift of the indirect NIID remained at $\sim 112 \mathrm{~nm}$ at annealing temperature of $650^{\circ} \mathrm{C}$ and $700^{\circ} \mathrm{C}$ and the PL linewidth was smaller than the as-grown and as-annealed samples [219]. These demonstrations of annealing effect on practical $p-i-n$ structures allowed realization of bandgap tuned lasers and provided insight into the technological capabilities of intermixing in monolithic integration of different devices for photonics in tegrated circuits.
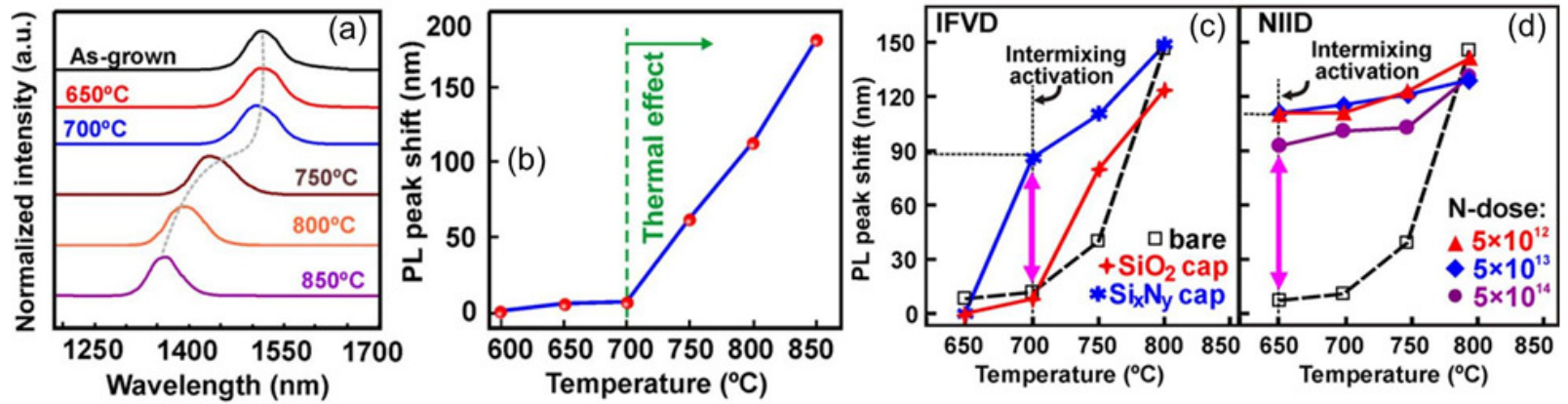

Fig. 31. (a) PL spectrum and (b) PL peak shift at $77 \mathrm{~K}$ taken under an excitation density of $3 \mathrm{~W} / \mathrm{cm}^{2}$, when the GS PL signal from InAs/InGaAlAs DaWELL samples is not saturated, versus annealing temperature, and capped with $200 \mathrm{~nm} \mathrm{SiO}$. (c) Peak shift of $77 \mathrm{~K}$ PL against annealing temperature using the following: (c) IFVD with $\mathrm{SiO}_{2}$ and $\mathrm{Si}_{x} \mathrm{~N}_{y}$ cap dielectric cap annealing, and (d) NIID at different doses. The dotted line in (c) and (d) indicates the activation temperature required to initiate the spatially selective intermixing. Taken from [219].

\subsection{Chirped Qdash active region design}

The large inhomogeneous broadening of self-assembled Qdash nanostructure active region demerits the laser performance since there is always a trade-off between the spectral linewidth and the minimum gain required for driving the device. In contrast, the flexibility offered by Qdash geometry and density has been exploited for realization of broad gain material for SOA and broadband light emitting devices. Various approaches were reported in literature to create additional Qdash inhomogeneity within the active region, usually referred as chirped designs. Deubert et al. [220] introduced this technique on Qdash platform by growing two device-structures; firstly, the 6-stack Qdash with varying InAs nominal thickness (3 to $4.6 \mathrm{ML}$ ), and fixed $25 \mathrm{~nm}$ barriers graded separate confinement heterostructure structure; and secondly, the 4-stack Qdash with identical 4 ML InAs deposition thickness in conjunction with the reduced barriers of $10 \mathrm{~nm}$ for strain coupling, and for modifying the Qdash size from layer to layer, as discussed earlier. Although PL measurement results were not reported, the laser device results below threshold showed a broad electroluminescence from both the structure suggesting an increase in gain-bandwidth by $45 \%$ compared to the conventional 4 stack Qdash laser structure with identical InAs layer thickness with thick barriers. In the same year, Reithmaier et al. [26] demonstrated a wide gain-bandwidth of $270 \mathrm{~nm}$ with peak gain $40 \mathrm{~cm}^{-1}$ at 5 $\mathrm{kA} / \mathrm{cm}^{2}$ from a chirped active region which is a combination of the above two designs i.e. 4 dash 
layers with different InAs thickness separated by fixed thin $10 \mathrm{~nm}$ barriers (design A) [221]. The measurement was done under pulsed current using segment contact method and shown in Fig. 32(a). In addition, another design named as $2 \times 2$ scheme (design B) wherein two groups of nominally identical Qdash layers, each separated by $10 \mathrm{~nm}$ barriers, and the groups separated by $20 \mathrm{~nm}$ barriers, was proposed and demonstrated. A gain-bandwid th of $210 \mathrm{~nm}$ was reported from this structure with peak gain of $10 \mathrm{~cm}^{-1}$ at pulsed current density of $5 \mathrm{kA} / \mathrm{cm}^{2}$ (see Fig. 32(a)).

Because of good tunability properties and control of Qdash emission, Somers et al. [222] was able to further extend the gain-bandwid th of Qdash structure to a value of $>300 \mathrm{~nm}$ with peak gain of $35 \mathrm{~cm}^{-1}$ which satisfies the need of broadband SOA covering the entire long wavelength communication band from $\sim 1.33 \mu \mathrm{m}$ to $\sim 1.67 \mu \mathrm{m}$ (see Fig. 32(b)). This is achieved by using 6-stack Qdash layers (design C) that was separated into three groups with $20 \mathrm{~nm}$ barrier in between, and the two Qdash layer within a group separated by $10 \mathrm{~nm}$ barrier. The nominal InAs layer thickness in each group was varied from $0.91 \mathrm{~nm}$ to $1.31 \mathrm{~nm}$ [5]. Very recently, our group demon strated a broad PL linewidth from a chirped InGaAlAs barrier 4-stack DaWELL laser structure [223] based on the systematic study on the effect of barrier thickness on the active region inhomogeneity [224]. By employing variable barrier thickness of $10 \mathrm{~nm}$ (p-side), $10 \mathrm{~nm}, 15 \mathrm{~nm}$, and $20 \mathrm{~nm}$ (n-side), we demonstrated an ultra-broad $77 \mathrm{~K}$ PL linewidth of $\sim 151 \mathrm{~nm}$ and found a significant increase in the linewidth when compared to a 4 stack fixed $10 \mathrm{~nm}$ barrier similar Qdash laser sample $(77 \mathrm{~K} \mathrm{PL}$ linewidth $\sim 100 \mathrm{~nm}$ ). We also performed gain measurements on the chirped barrier thickness Qdash structure utilizing the segment contact method, and measured a broad gain-bandwid th of $\sim 140 \mathrm{~nm}$ under $\mathrm{CW}$ current density of $8.3 \mathrm{kA} / \mathrm{cm}^{2}$ with peak modal gain $\sim 41 \mathrm{~cm}^{-1}$ [225], as illustrated in Fig. $32(\mathrm{c})$.
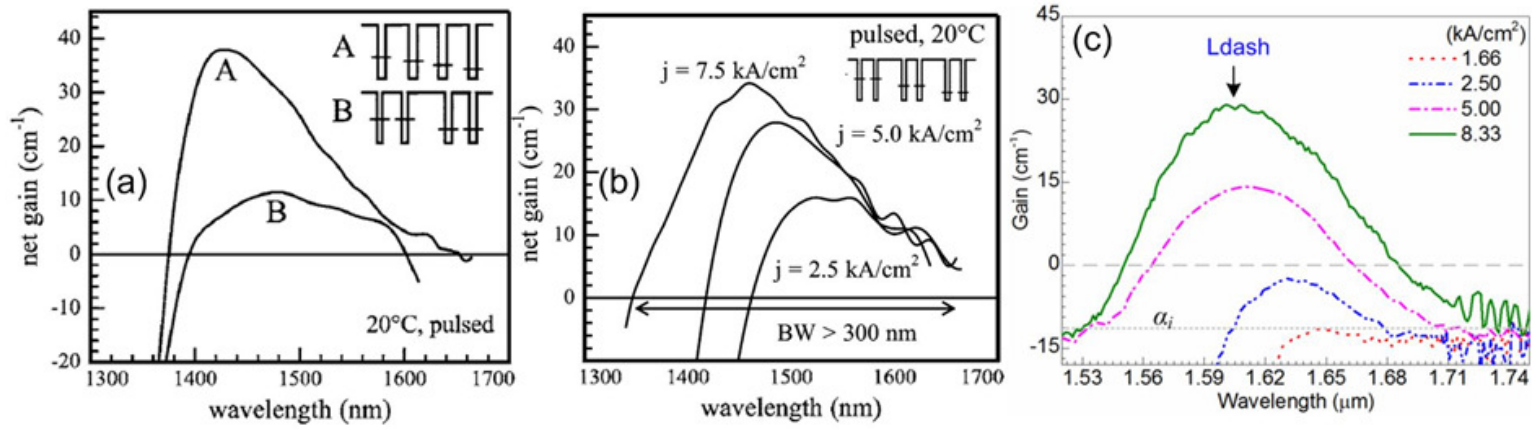

Fig. 32. Pulsed room temperature modal net gain of a InAs/InGaAlAs SCH Qdash laser structure with three broadened gain de signs: (a) first (design A) consisting of four Qdash layers with different Qdash sizes (InAs deposition thickness) and the Qdash layers are separated by $10 \mathrm{~nm}$ thick barriers; second (design B) consisting of two Qdash layer groups of different nominal Qdash layer thicknesses, and the individual Qdash layers are separated by $10 \mathrm{~nm}$ and the groups by $20 \mathrm{~nm}$, respectively; (b) third (design C) is a six layer three Qdash group structure combining designs A and B, as shown in the inset. (c) Gain of a four stack DaWELL laser structure with chirped barrier layer thickness (10, 10, 15, $20 \mathrm{~nm})$. Adapted from [222, 225]. 


\section{InAs/InP Qdash Devices}

In this section, we reviewed the accomplishments of Qdash active region in semiconductor devices by discussing the edge-emitting FP lasers, mode-locked lasers, SOA, and the recent new class of broadband lasers. Unlike conventional lasers, which emit narrow linewidth coherent beam, the broadband laser produces stimulated emission with much broader spectral linewidth. In addition, In addition, we also summarized utilization of Qdash active medium as SLDs in which our group pioneered.

\subsection{InAs/InP Qdash lasers}

The first demonstration of semiconductor diode laser employing Qdash active region was reported by Wang et al. [3] at room temperature using InAs/InGaAlAs Qdash material system on (100) InP substrate. This founding work led to the realization of the technological capabilities of this nanostructure, particularly in the field of mode-locking and optical amplification. The natural selfassembled growth of Qdashes on InP substrates without any assisting growth techniques makes it as a favorite contender for realizing long wavelength semiconductor devices.

\subsubsection{Lasers on (100) InP substrate}

\section{InAs/InGaAlAs Material System}

Fig. 33(a) shows the room temperature lasing from 1-, 3-, and 5-stack InAs/InGaAlAs DaWELL structure, reported first by Wang et al. [3] under pulsed current operation, emitting at $1.60 \mu \mathrm{m}$, $1.62 \mu \mathrm{m}$, and $1.66 \mu \mathrm{m}$, respectively. The correspond ing threshold current densities were $410 \mathrm{~A} / \mathrm{cm}^{2}$, $500 \mathrm{~A} / \mathrm{cm}^{2}$ (167 A/cm ${ }^{2}$ per layer), and $766 \mathrm{~A} / \mathrm{cm}^{2}$ (154 A/cm ${ }^{2}$ per layer), obtained from $100 \mu \mathrm{m}$ stripe broad-area lasers with cavity length $1.5-4.0 \mathrm{~mm}$ (the cavity was perpendicular to Qdash elongation). The internal quantum efficiency of $\sim 0.6$ and internal loss $\sim 10 \mathrm{~cm}^{-1}$ was extracted and high modal gain of $\sim 15 \mathrm{~cm}^{-1}$ from the single stack laser diode. These encouraging results motivated the research community to pursue further on this material system. Next report came from Schwertberger et al. [181] who reported wavelength tuning of InAs/InGaAlAs Qdash lasers from $1.54 \mu \mathrm{m}$ to $1.78 \mu \mathrm{m}$ by just varying the InAs deposition thickness from 5.0 ML to 7.5 ML. A threshold current density as low as $900 \mathrm{~A} / \mathrm{cm}^{2}\left(225 \mathrm{~A} / \mathrm{cm}^{2}\right.$ per layer) from $40 \times 1000 \mu \mathrm{m}^{2}$ device was reported with a total output power $>100 \mathrm{~mW}$ and characteristics temperature $\mathrm{T}_{0} \sim 61 \mathrm{~K}\left(20-80^{\circ} \mathrm{C}\right)$. The results are summarized in Fig. 33(b). Subsequently, with the improvement in the material quality, indicated by the improved quantum efficiency of $\sim 0.6$ and internal loss $\sim 8.5 \mathrm{~cm}^{-1}$ [180], a reduced transparency current density of $350 \mathrm{~A} / \mathrm{cm}^{2}$ (90 A/cm ${ }^{2}$ per layer) was reported, which is the among the best value reported in this material system. In addition, a $3 \times 2000 \mu \mathrm{m}^{2}$ ridge-waveguide lasers at $\sim 1.55 \mu \mathrm{m}$ was also demonstrated with an output power $>30 \mathrm{~mW}$ at room temperature. The flexibility in tuning the emission wavelength of Qdashes was shown by Rotter et al. [182] who demonstrated a lasing emission from a 5-stack InAs Qdashes in InGaAs Qwell laser structure on (100) InP at $2.03 \mu \mathrm{m}$, which is the record longest wavelength ever reported on any InAs/InP Qdash or Qdots material system. A pulsed threshold current density of $540 \mathrm{~A} / \mathrm{cm}^{2}\left(108 \mathrm{~A} / \mathrm{cm}^{2}\right.$ per layer) was reported with $\sim 0.52$ internal quantum efficiency, $\mathrm{T}_{0}=41 \mathrm{~K}$, and a very high internal loss $\sim 55$ $\mathrm{cm}^{-1}$ which was attributed to Rayleigh scattering of the laser mode due to local undulations of the refractive index near the Qdashes.

Following Rotter et al.'s work, the potential of this nanostructure to cover significantly longer wavelength range was further affirmed by Somers et al. [221] who reported $1.88 \mu \mathrm{m}$ lasing emission from InAs/InGaAs DaWELL lasers with good device performance ( $\sim 0.6$ internal quantum efficiency and $\sim 4 \mathrm{~cm}^{-1}$ internal loss), as shown in Fig. 33(c). Room temperature CW operation of 4 stack graded SCH InAs/InGaAlAs Qdash lasers was reported by Resneau et al. [226] who developed $1.57 \mu \mathrm{m}$ ridge-waveguide lasers with total power $>100 \mathrm{~mW}$ and a flat low RIN of $-162 \mathrm{~dB} / \mathrm{Hz}$ in 
0.1-13 GHz range (lower than the Qwell laser counterpart). The extracted small internal loss of 4 $\mathrm{cm}^{-1}$ from cavity length dependence measurements of the external quantum efficiency is among the smallest reported in this material system. In addition, dynamic characteristics measurements revealed a modulation efficiency of $0.36 \mathrm{GHz} / \mathrm{mA}^{0.5}$, K-factor of $1.51 \mathrm{~ns}$ and maximum intrinsic bandwidth of $5.9 \mathrm{GHz}$. These values were further improved in their following work [26] ([227]) with 0.64 (0.72) $\mathrm{GHz} / \mathrm{mA}^{0.5}$ modulation efficiency and $5.2(7.5) \mathrm{GHz}$ resonance frequency value, which was also based on non-optimized Qdash laser design for high speed measurements. The small signal modulation bandwidth in the former case was $5.6 \mathrm{GHz}$.
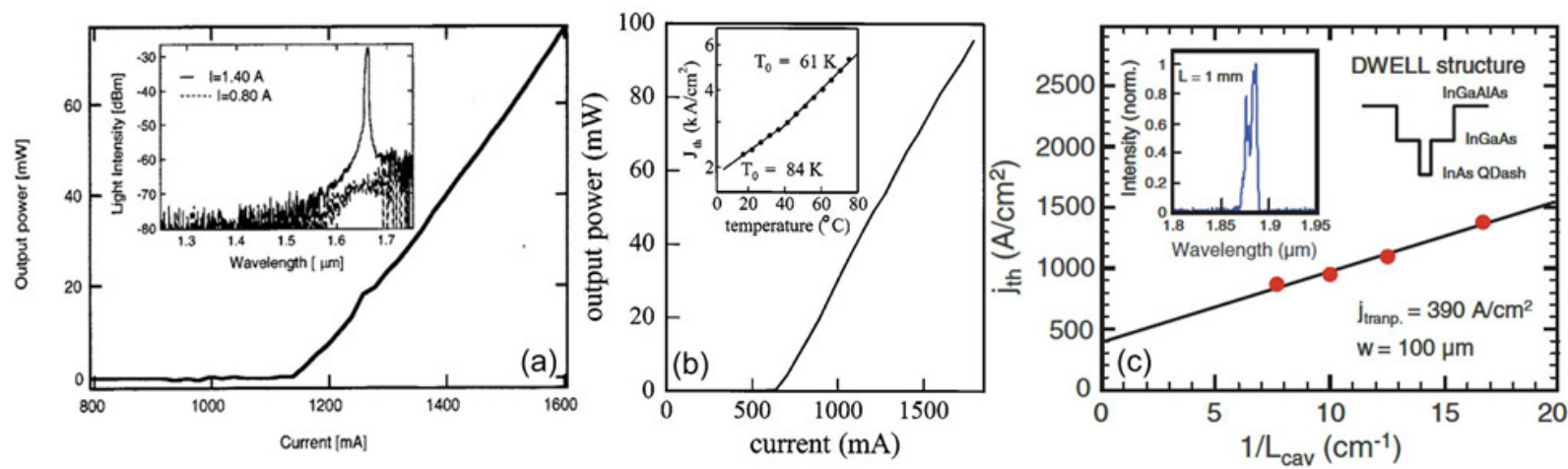

Fig. 33. (a) A two-facet light output versus pulsed injection current measured on a five-stack Qdash laser diode with geometry of $100 \times 1500 \mu \mathrm{m}^{2}$. The inset in (a) is the electroluminescence spectra under different pump levels for the device. (b) Single facet light output versus pulsed injection current of a $40 \times 500 \mu \mathrm{m}^{2}$ Qdash laser emitting at $1.54 \mu \mathrm{m}$. The inset in (b) shows the temperature dependence of the thre shold current density with solid lines representing the linear fits to the data points. (c) Threshold current density of a long wavelength broad area Qdash laser with a DaWELL design versus inverse cavity length. The inset in (c) shows the lasing emission spectrum of a $1000 \mu \mathrm{m}$ long device at $1.88 \mu \mathrm{m}$. Taken from $[3,26,181]$

The thrust to improve the high speed performance of Qdash lasers lead to the realization of $\mathrm{p}$ doped [228, 229] and tunnel injection schemes [186, 188]. Mi et al. [188] reported a high $\mathrm{T}_{0} \sim 196 \mathrm{~K}$ (up to $40^{\circ} \mathrm{C}$ ), enhanced small signal modulation bandwidth of $8 \mathrm{GHz}$ at $5^{\circ} \mathrm{C}$, sub-threshold $\mathrm{LEF} \sim 1$ and $<1.5$ frequency chirp, by employing a modulation p-doped 6-stack InAs/InGaAlAs Qdash laser under pulsed current operation, as shown in Figs. 34(a) and (b). This improvement was attributed to the increase in gain and differential gain of the active region. However, the internal loss increased substantially $\left(15.5 \mathrm{~cm}^{-1}\right)$ due to p-doping induced increased Auger recombination but a good internal efficiency of $\sim 0.6$ was maintained. Later, Hein et al. [228] demonstrated a CW $8 \mathrm{GHz}$ small signal modulation bandwidth with $0.76 \mathrm{GHz} / \mathrm{ma}^{0.5}$ modulation efficiency at room temperature from a moderately p-doped $3 \times 580 \mu \mathrm{m}^{2}$ ridge-waveguide 4-stack Qdash laser with exceptionally high slope efficiency 0.4 W/A at $1.55 \mu \mathrm{m}$. A systematic and detailed investigation of the effect of p-doping concentration on the Qdash lasers revealed [229] that high p-doping concentrations increased the differential gain by more than $50 \%$ but the enhanced gain compression and enlarged thermal heating due to high internal losses overcompensated this benefit. Therefore, a maximum small signal modulation bandwidth of $8 \mathrm{GHz}$ in $\mathrm{CW}$ operation at room temperature was obtained for moderate $\mathrm{p}$ doping concentration $\left(\sim 10\right.$ holes per Qdash) with internal loss $11 \mathrm{~cm}^{-1}, \sim 0.76$ internal quantum efficiency, and $\mathrm{T}_{0} \sim 83 \mathrm{~K}\left(20\right.$ to $\left.80^{\circ} \mathrm{C}\right)$. A maximum $\mathrm{T}_{0} \sim 96 \mathrm{~K}\left(20\right.$ to $\left.80^{\circ} \mathrm{C}\right)$ and modal gain $\sim 41 \mathrm{~cm}^{-1}$ were reported for 100 holes per Qdash p-doping but with an increased internal loss and transparency current density of $28 \mathrm{~cm}^{-1}$ and $1.3 \mathrm{kA} / \mathrm{cm}^{2}$, respectively. By employing tunnel injection scheme in tandem with p-doping, Mi et al. [186] succeeded in pushing the small signal modulation bandwidth up to $12 \mathrm{GHz}$ at $5^{\circ} \mathrm{C}$, which is the best value reported in InAs/InP Qdash system, with an enhanced $\mathrm{T}_{0} \sim 204 \mathrm{~K}$ (up to $40^{\circ} \mathrm{C}$ ) and reduced sub-threshold $\mathrm{LEF}<0.7$. This was attributed to the combined effects of p-doping and tunnel injection which minimized the hot carrier effects and carrier leakage in the active region. On the other hand, Hein et al. [230] reported a room temperature maximum small signal bandwidth of $9.6 \mathrm{GHz}$ utilizing $2.5 \times 600 \mu \mathrm{m}^{2}$ undoped 6-stack InAs/InGaAlAs Qdash laser in $\mathrm{CW}$ operation with a modulation efficiency of $0.82 \mathrm{GHz} / \mathrm{mA}^{0.5}, \mathrm{~K}$-factor of $0.78 \mathrm{~ns}$ and maximum intrinsic bandwidth of $11.4 \mathrm{GHz}$, as illustrated in Fig. 34(c). This is the best value reported in Qdash system at room temperature. The corresponding above-threshold LEF of 2.5 was obtained 
utilizing the amplitude-modulation/frequency-modulation method. In another study, Dziak et al. [231] also measured the above-threshold LEF of Qdash laser using the injection locking scheme. In this case, the measured LEF increased from 1.2 to 8.6 with increasing current injection at the peak lasing wavelength.
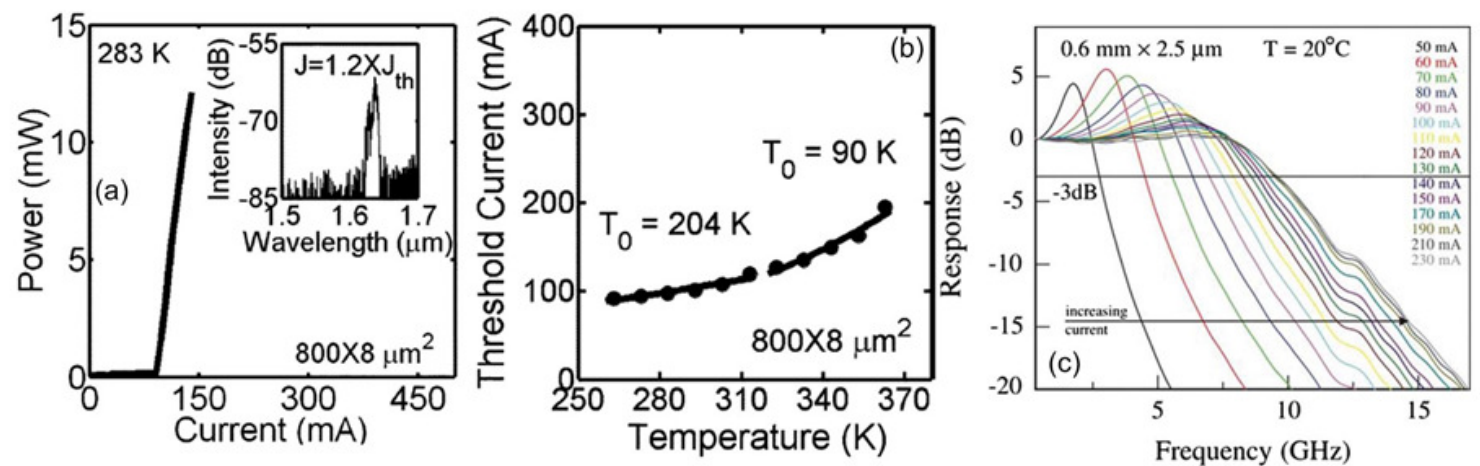

Fig. 34. Characteristics of p-doped InAs tunnel injection InAs/InGaA 1 As Qdash lasers under pulsed operation: (a) $L-I$ and output spectrum (inset) and (b) variation of threshold current $w$ ith temperature. (c) Room temperature small-signal modulation response of a ridge-waveguide $2.5 \times 600 \mu m^{2}$ undoped InAs/InGaAlAs Qdash laser for various CW drive currents. Reproduced from [186, 230].

The best multi-stack Qdash laser threshold current density value $\left[730 \mathrm{~A} / \mathrm{cm}^{2}\left(46 \mathrm{~A} / \mathrm{cm}^{2}\right.\right.$ per layer)] on this material system was reported by Hein et al. [189] from $1.3 \mathrm{~mm}$ long 16-stack columnar Qdash laser structure emitting at $1.7 \mu \mathrm{m}$ followed by Rotter et al. [182] [540 A/cm ${ }^{2}(108$ $\mathrm{A} / \mathrm{cm}^{2}$ per layer)]. In terms of device internal quantum efficiency, our group reported the best value of 0.93 for the 4-stack Qdash laser [216]. In addition, our group successfully showed the improvement in the Qdash laser performance by controlled IFVD intermixing process with $\sim 11 \%$ and $\sim 45 \%$ reduction in the threshold current and transparency current density, respectively, while maintaining the other laser performance parameter values similar, and lasing at $\sim 1.61 \mu \mathrm{m}$. This was attributed to the defect annealing at the Qdash-Qwell interfaces in the 4-stack InAs/InGaAlAs Qdash laser.

Chirped active region based Qdash lasers were also reported in this material system by various groups, and their designs has already been discussed in section 4.4. In terms of device performance Deubert et al. [220] and Somers et al. [221, 222] reported lasing at $1.53 \mu \mathrm{m}$ with reasonable threshold current density (250-440 A/ $\mathrm{cm}^{2}$ per layer), internal quantum efficiency (0.60.75 ) and internal loss $\left(4.6-10 \mathrm{~cm}^{-1}\right)$. On the other hand, our chirped Qdash lasers exhibited 550 $\mathrm{A} / \mathrm{cm}^{2}$ per layer threshold current density with 0.85 and $11 \mathrm{~cm}^{-1}$ internal quantum efficiency and internal loss, respectively [225]. Lastly, the reliability properties of the strained InAs/In GaAlAs Qdash lasers were also reported by Resneau et al. [232] because of their susceptibility to the creation of crystal defects. Lifetime measurements under CW operation on many $0.9 \mathrm{~mm}$ [227] and $1.9 \mathrm{~mm}$ [26] long Qdash lasers were carried out over $2800 \mathrm{~h}$ and $7000 \mathrm{~h}$, respectively, at $60^{\circ} \mathrm{C}$. A stable average output power of $10 \mathrm{~mW}$ per facet was observed with slight improvement in power after the first 100-200 h of burn-in time in the longer laser cavity cases.

\section{$\underline{\text { In As/InGaAsP Material System }}$}

Qdash lasers based on this material system were embraced relatively late with Moreau et al. [233] reporting the effect of layer stacking and p-doping on the performance of $1.52 \mu \mathrm{m} \mathrm{InAs/InGaAsP}$ Qdash lasers on (100) InP substrate, as depicted in Fig. 35(a). A pulsed threshold current density as low as $1100 \mathrm{~A} / \mathrm{cm}^{2}\left(123 \mathrm{~A} / \mathrm{cm}^{2}\right.$ per layer) from $50 \times 600 \mu \mathrm{m}^{2}$ device with 9 -stack InAs/InGaAsP DaWELL structure was reported at room temperature. The corresponding transparency current density was $83 \mathrm{~A} / \mathrm{cm}^{2}$ per layer and high modal gain of $48 \mathrm{~cm}^{-1}\left(5.4 \mathrm{~cm}^{-1}\right.$ per layer). These values were found to be superior when compared to the 6-stack and 12-stack Qdash laser performances. In general, the characteristics temperature $\mathrm{T}_{0}$ measured from these different stack lasers were in the range of $\sim 60-70 \mathrm{~K}\left(20\right.$ to $\left.80^{\circ} \mathrm{C}\right)$ with internal loss $\sim 14 \mathrm{~cm}^{-1}$. On the other hand, p-doping at different 
doping concentration on the 6-stack sample revealed insignificant improvement in characteristics temperature $\mathrm{T}_{0} \sim 60 \mathrm{~K}$ as observed in Ref. [186] with substantially increased internal loss $25-46 \mathrm{~cm}^{-}$ 1. These demonstrations were soon followed by room temperature ridge-waveguide Qdash laser operation report lasing at $1.51 \mu \mathrm{m}$ with output power $>14 \mathrm{~mW}$ and slope efficiency 0.4 W/A [233235]. The above-threshold LEF values which is in the range of 3.6-6.5 was found to increase with current injection, similar to other reports on InAs/InGaAlAs system [231]. This was attributed to the plasma effect and the carrier filling of the non-lasing states (higher transition energy states and the wetting layer), which resulted in reduction of differential gain above threshold. In another study, Lelarge et al. [207] compared the Qdash-in-barrier and DaWELL scheme at room temperature on $50 \times 2000 \mu \mathrm{m}^{2}$ device under CW operation and observed a red shift in the lasing wavelength $(1.56 \mu \mathrm{m}$ in the former scheme while $1.65 \mu \mathrm{m}$ in the latter scheme). In addition, the transparency current density, plotted in Fig. 35(b), was shown to significantly decrease in the DaWELL scheme with 660 $\mathrm{A} / \mathrm{cm}^{2}$ (110 A/cm ${ }^{2}$ per layer) compared to the Qdash-in-barrier scheme $1140 \mathrm{~A} / \mathrm{cm}^{2}\left(190 \mathrm{~A} / \mathrm{cm}^{2}\right.$ per layer), due likely to better carrier injection [236]. An internal quantum efficiency of 0.8 with an estimated internal loss of $19 \mathrm{~cm}^{-1}$ from the DaWELL structures was reported. In addition, a modal gain as high as $105 \mathrm{~cm}^{-1}$ (from short $140 \mu \mathrm{m}$ cavity) and characteristics temperature $\mathrm{T}_{0} \sim 60 \mathrm{~K}$ (up to $80^{\circ} \mathrm{C}$ ) were extracted. It was shown that even the p-doping in the active region did not increase $\mathrm{T}_{0}$ which was attributed either to the shape of the nanostructures or to the band structure.
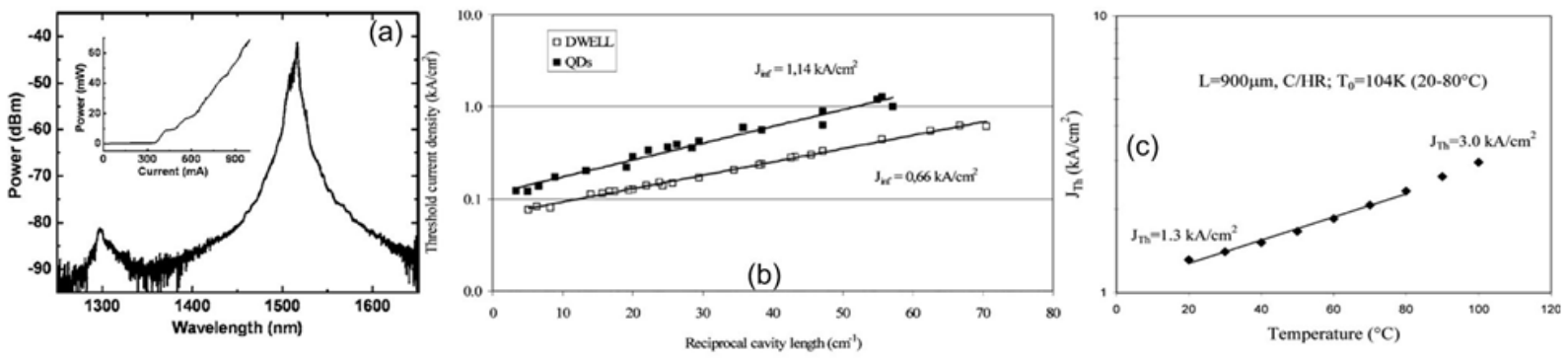

Fig. 35. (a) Room temperature above thre shold lasing spectrum under pulsed excitation of InAs/InGaAsP DWELL broad area laser. The inset in (a) shows the $L-I$ characteristic of the $40 \times 518 \mu \mathrm{m}^{2}$ de vice. (b) Threshold current density of $50 \mu \mathrm{m}$ broad area lasers versus reciprocal cavity length for InAs/InGaAsP dash-in-a-barrier and dash-in-a-well struc tures. (c) Threshold current density variation with temperature of an optimized $50 \times 900 \mu m^{2}$ dash-in-well broad area lasers with re duced InGaAsP Qwell thic kness of 6nm. Collected from [207, 233]

Later, through high quality growth optimization of Qdashes and utilizing buried ridge fabrication process, a high $\mathrm{T}_{0} \sim 135 \mathrm{~K}\left(25\right.$ to $\left.85^{\circ} \mathrm{C}\right)$ was reported from p-doped 6-stack Qdash-inbarrier laser with two times higher modal gain compared to the undoped laser $\left[66 \mathrm{~cm}^{-1}\left(11 \mathrm{~cm}^{-1}\right.\right.$ per layer) versus $34 \mathrm{~cm}^{-1}\left(5.6 \mathrm{~cm}^{-1}\right.$ per layer)], but with high threshold $\left(\sim 10 \mathrm{kA} / \mathrm{cm}^{2}\right)$ and internal loss $\left(\sim 60 \mathrm{~cm}^{-1}\right)$ [237]. Moreover, a significant improvement in the device dynamic characteristics was also reported with a high relaxation frequency value of $13.5 \mathrm{GHz}$ obtained from the RIN measurements $(-155$ to $-160 \mathrm{~dB} / \mathrm{Hz}$ from 0.5 to $20 \mathrm{GHz})$; among the best values reported on In As/InP Qdash material system. However, the p-doped device showed an inferior measured small signal modulation bandwidth value of $7 \mathrm{GHz}$ due mostly to $\mathrm{RC}$ parasitic limitation related to the buried ridge process. But, a strong decrease in the above-threshold LEF $<3.5$ for current up to 140 $\mathrm{mA}$ and a successful large signal modulation capability at 10 Gbps utilizing NRZ PBRS signal affirmed the p-doped Qdash laser compatibility for $10 \mathrm{Gbps} \mathrm{CW}$ operation.

Alternatively, Lelarge et al. [207] demonstrated an increase in $\mathrm{T}_{0}$ to $100 \mathrm{~K}$ (20 to $\left.80{ }^{\circ} \mathrm{C}\right)$, as shown in Fig. 35(c) without employing any p-doping in the active region, and jut by optimized active region design with a thin InGaAsP Qwell layer of $6 \mathrm{~nm}$. This was attributed to an increased energy level of the ES of the DaWELL structure which reduced the carrier escape from Qdash to Qwell. This slight modification in the active region design was further attested by ridge-waveguide (buried ridge stripe) $1.5 \times 600 \mu \mathrm{m}^{2}$ Qdash laser demonstration under $\mathrm{CW}$ operation. A characteristic temperature $\mathrm{T}_{0} \sim 80 \mathrm{~K}$ (up to $80{ }^{\circ} \mathrm{C}$ ) with threshold current density as low as $12 \mathrm{~mA}$ and lasing wavelength $\sim 1.55 \mu \mathrm{m}$ was reported. This Qdash active region design was further optimized by Dagens et al. [238] by reducing p-side $(20 \mathrm{~nm})$ and $\mathrm{n}$-side $(70 \mathrm{~nm}) \mathrm{SCH}$ layers for the 6-stack In As/InGaAsP Qdash laser in order to reduce the carrier transit time, and to limit the recovery of the 
optical mode and the absorbing p-doped waveguiding layers. A $600 \mu \mathrm{m}$ long buried ridge-waveguide laser demonstrated a relaxation frequency of about $8.5 \mathrm{GHz}$, relatively flat RIN $-155 \mathrm{~dB} / \mathrm{Hz}(0.1-16$ $\mathrm{GHz}$ ), and a small signal modulation bandwidth of $10.5 \mathrm{GHz}$ in $\mathrm{CW}$ operation and room temperature (largest value ever reported on any Qdash material system), as shown in Figs. 36(a) and (b). The laser output power was $>25 \mathrm{~mW}, \mathrm{~T}_{0} \sim 72 \mathrm{~K}\left(20\right.$ to $\left.80{ }^{\circ} \mathrm{C}\right)$ and $\mathrm{CW}$ threshold current of $32 \mathrm{~mA}$. Furthermore, investigation of the large signal modulation characteristics on $300 \mu \mathrm{m}$ long device utilizing 10 Gbps NRZ PRBS signal and measuring the BER after passing through 10 Gbps synchronous digital hierarchy $(\mathrm{SDH})$ receiver demonstrated a floor free BER with $10^{-10}$ sensitivity of $-11 \mathrm{dBm}$ with $7 \mathrm{~dB}$ extinction ratio at the laser output. Even at elevated temperatures of $75^{\circ} \mathrm{C} \mathrm{a}$ successful floor-free back-to-back BER with $10^{-10}$ sensitivity of $-9.6 \mathrm{dBm}$ at $10 \mathrm{Gbps}$ was reported by Lelarge et al. [207]. P-doping on this optimized active region design was also performed and reported via fabrication of the DFB lasers [239] which is discussed in section 5.1.4.

Tunneling injection scheme was also investigated by Lelarge et al. [240, 241] on the In As/InGaAsP Qdash laser material system. A threshold current density of $2000 \mathrm{~A} / \mathrm{cm}^{2}\left(334 \mathrm{~A} / \mathrm{cm}^{2}\right.$ per layer) was obtained from the optimized $50 \times 600 \mu \mathrm{m}^{2}$ laser device under $\mathrm{CW}$ operation and the transparency current density as low as $155 \mathrm{~A} / \mathrm{cm}^{2}$ per layer. A buried rid ge-waveguide configuration incorporating the tunneling scheme was later utilized to test the dynamic properties. The $1.5 \times 600$ $\mu \mathrm{m}^{2}$ optimized laser device exhibited a threshold current of $16 \mathrm{~mA}$ with slope efficiency $0.34 \mathrm{~W} / \mathrm{A}$, output $>10 \mathrm{~mW}$ and $\mathrm{T}_{0} \sim 50 \mathrm{~K}\left(20\right.$ to $\left.80^{\circ} \mathrm{C}\right)$. The room temperature RIN measurements resulted in a value of $-155 \mathrm{~dB} / \mathrm{Hz}$ from $0.1-16 \mathrm{GHz}$ range with an extracted resonance frequency $\sim 8.5 \mathrm{GHz}$, and measured small signal modulation bandwidth of about $4.5 \mathrm{GHz}$, which showed strong parasitic-like roll off related to carrier transport limitations [240]. This insignificant effect of tunneling scheme on the laser dynamic characteristics was related mainly to the large escape of carriers from the injectorQdash ensembles which could be improved via higher energy barriers or moderate p-doping as has been demonstrated by Mi et al. [186] utilizing both p-doping and tunnel injection scheme.
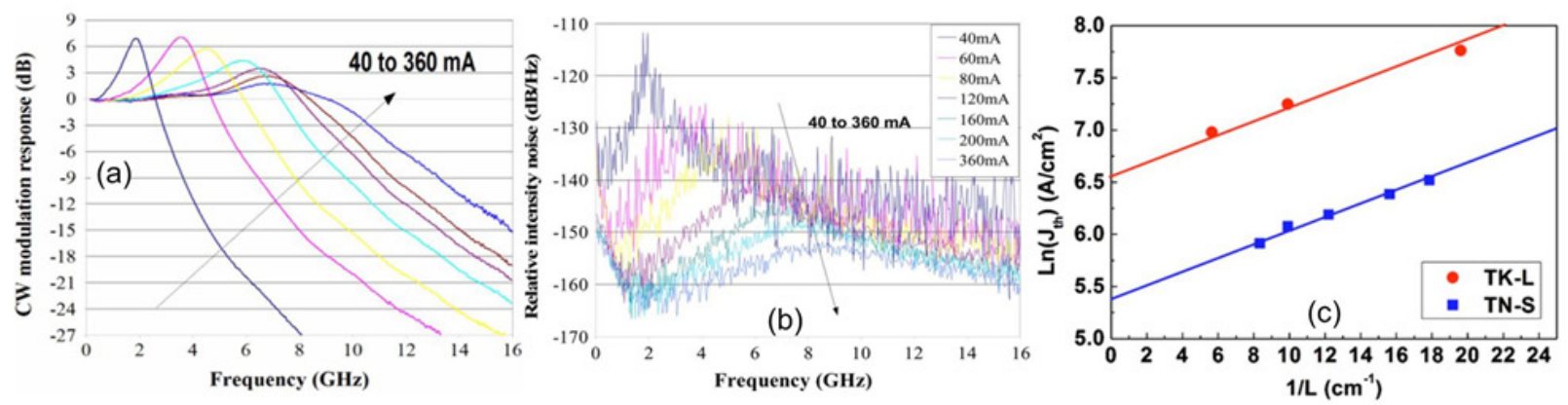

Fig. 36. (a) Small signal modulation response on a $600 \mu \mathrm{m}$ as-cleaved, reduced SCH layer thickness buried ridge stripe DaWELL laser at $25^{\circ} \mathrm{C}$ and (b) the corres ponding RIN measurement. (c) Threshold current density versus the reciprocal cavity length for 5-stack Qdash laser samples TN-S (TK-L) with $2.2 \mathrm{~nm}$ and $2.5 \mathrm{~nm}$ cap, and the corresponding $30 \mathrm{sec}$ and $60 \mathrm{sec}$ growth interruption. Solid lines in (c) are exponential fits of experimental re sults. Adapted from $[238,242]$.

In terms of the laser static performance, Zhou et al. [6, 242] demonstrated the lowest threshold current density of $360 \mathrm{~A} / \mathrm{cm}^{2}\left(72 \mathrm{~A} / \mathrm{cm}^{2}\right.$ per layer) and transparency current density as low as $220 \mathrm{~A} / \mathrm{cm}^{2}$ ( $45 \mathrm{~A} / \mathrm{cm}^{2}$ per layer) from 5-stack Qdash-in-barrier SCH laser structure grown by the optimized double-cap technique, and utilizing $2.2 \mathrm{~nm}$ cap with $30 \mathrm{sec}$ growth interruption (TN-S), as shown in Fig. 36(c). These are the best values reported on InAs/In GaAsP Qdash material system with extracted internal quantum efficiency and internal loss of 0.58 and $7 \mathrm{~cm}^{-1}$, respectively, and lasing at $\sim 1.55-1.58 \mu \mathrm{m}$. In addition, a detailed study on the effect of stacking layers on the Qdash laser performance was also performed [7,243] with demonstrated modal gain of $8 \mathrm{~cm}^{-1}$ and a red shift in the lasing wavelength (from 1.48 to $1.58 \mu \mathrm{m}$ ) on increasing the stacks from 2 to 6 . This was ascribed to the carrier re-distribution between multiple layers of inhomogeneous Qdashes. The high modal gain of Qdash lasers, corroborated via lasing from very short cavity lasers, has demonstrated in various reports $[5,26,207,244]$. In particular, a $120 \mu \mathrm{m}$ cavity exhibiting lasing with highest exhibited slope efficiency of 0.5 W/A was reported by Merghem et al. [245]. The $2 \times 120 \mu \mathrm{m}^{2} 6$-stack 
SCH Qdash laser device exhibited a threshold current density of $2.5 \mathrm{kA} / \mathrm{cm}^{2}$, lasing at $1.55 \mu \mathrm{m}$, output power $>5.0 \mathrm{~mW}$, and $34 \mathrm{~cm}^{-1}$ modal gain. This was followed by a detailed optimization of the modal gain by employing undoped multi-stack DaWELL structures and also introducing pdoping in the active region. A modal gain as high as $60 \mathrm{~cm}^{-1}$ was reported by Merghem et al. from a 15 stack undoped and 6 stack p-doped DaWELL structure [246]. Also, a small above-threshold LEF of $<2.4$ (up to $150 \mathrm{~mA}$ ) was measured from these devices with extracted resonance frequency of $>$ $10 \mathrm{GHz}$ from the RIN measurements, and was ascribed to the increase in the device differential gain. Next, Faugeron et al. [247] demonstrated the lowest internal loss of $2.7 \mathrm{~cm}^{-1}$ (on any Qdash material system) with high internal quantum efficiency of 0.81 utilizing an asymmetric-cladding 6-stack Qdash-in-barrier laser. In general, a ridge-waveguide Qdash lasers under the pulsed mode showed a threshold current density of $\sim 4 \mathrm{kA} / \mathrm{cm}^{2}$, slope efficiency $0.32 \mathrm{~W} / \mathrm{A}$, and lasing around $1.59 \mu \mathrm{m}$. In this material system also ageing test of InAs/In GaAsP Qdash lasers was performed by Resneau et al. [248] at $70^{\circ} \mathrm{C}$ and $90^{\circ} \mathrm{C}$ with an output power maintained at $10 \mathrm{~mW}$ for the $0.9 \mathrm{~mm}$ cavity laser. Sudden failure and change in the optical power were not observed from any of the 10 devices tested after $700 \mathrm{~h}$, thus showing the optical quality of these lasers.

\subsubsection{Multiwavelength Qdash lasers}

Exploiting the inherent size dispersion of the self-assembled Qdashes resulting in a broadband gain spectrum is advantageous for comb generation, i.e. equally spaced multiwavelength generation with a precise channel separation, attractive for wavelength division multiplexed (WDM) system. Akrout et al. [249] was the first to exploit this feature from a $420 \mu \mathrm{m}$ long buried ridge-waveguide InAs/InGaAsP FP Qdash laser which provided a flat optical spectrum with $\sim 12$ channels and 100 $\mathrm{GHz}$ channel spacing, centered at $1.55 \mu \mathrm{m}$, as shown in Fig. 37(a), by exploiting the inherent FP mode spacing. The error free $\left(\mathrm{BER}<10^{-9}\right)$ transmission through a single mode fiber over $50 \mathrm{~km}$, utilizing 8 separate on-off keying channels at $10 \mathrm{Gbps}$, was demonstrated at a power level of $\sim-16$ $\mathrm{dBm}$ (see Fig. 37(b)). A penalty of $1.5 \mathrm{~dB}$ was measured compared to a reference single mode external laser source and was attributed to the rise in the RIN level of the filtered modes $(\sim-110$ $\mathrm{dB} / \mathrm{Hz}$ from $0.1-20 \mathrm{GHz})$ [250]. This work was further strengthened by M'Sallem et al. [251] demonstrating multiwavelength Qdash laser source for WDM multicast applications with differential phase-shift keying (DQPSK). An error free transmission of 56 Gbps DQPSK modulation on 9 channels with $100 \mathrm{GHz}$ spacing [corresponding to the International Telecommunication Union (ITU) grid channel spacing] was achieved. An error floor at BER of $10^{-9}$ was obtained with a power penalty from 1.5 to $5 \mathrm{~dB}$, as illu strated in Fig. 37(c). In another study, Nguyen et al. [252] employed Qdash mode-locked laser as a multiwavelength coherent seeding source for colorless WDM-passive optical network (PON) system based on injection locked FP laser diode. An error free transmission at 2.5 Gbps was achieved over $25 \mathrm{~km}$ single mode fiber with 16 channels operating in the C-band with 85 GHz spacing. An average BER sensitivity of $10^{-9}$ at receiver input power $-21.9 \mathrm{~dB}$ was reported with a power penalty of $>0.5 \mathrm{~dB}$. The suitability of Qdash multiwavelength laser for future coherent orthogonal frequency division multiplexing (OFDM) super channel applications was demonstrated by Rosales et al. [253] who assessed the mode coherence between spectral modes of a multiwavelength $890 \mu \mathrm{m}$ long Qdash laser diode. At $400 \mathrm{~mA}$ bias current the group delay and spectral phase of the modes were determined and a group dispersion delay of $1.3 \mathrm{ps}^{2}$ was calculated. When the modes passed through a $65 \mathrm{~m}$ single mode fiber (to compensate the group dispersion delay), mode locking was observed. Next, the single side band OFDM signal, which constituted 74 sub-carriers each encoded with 16-QAM, was modulated into 33 modes of the $48 \mathrm{GHz}$ Qdash laser, with a spectral bandwidth of $\sim 1.5 \mathrm{THz}$ [254]. However, the coherent transmission required an optical linewidth of $<1 \mathrm{MHz}$, which require further optimization of the Qdash mode-locked lasers as currently a typical linewidth of about few to tens of $\mathrm{MHz}$ is available. 

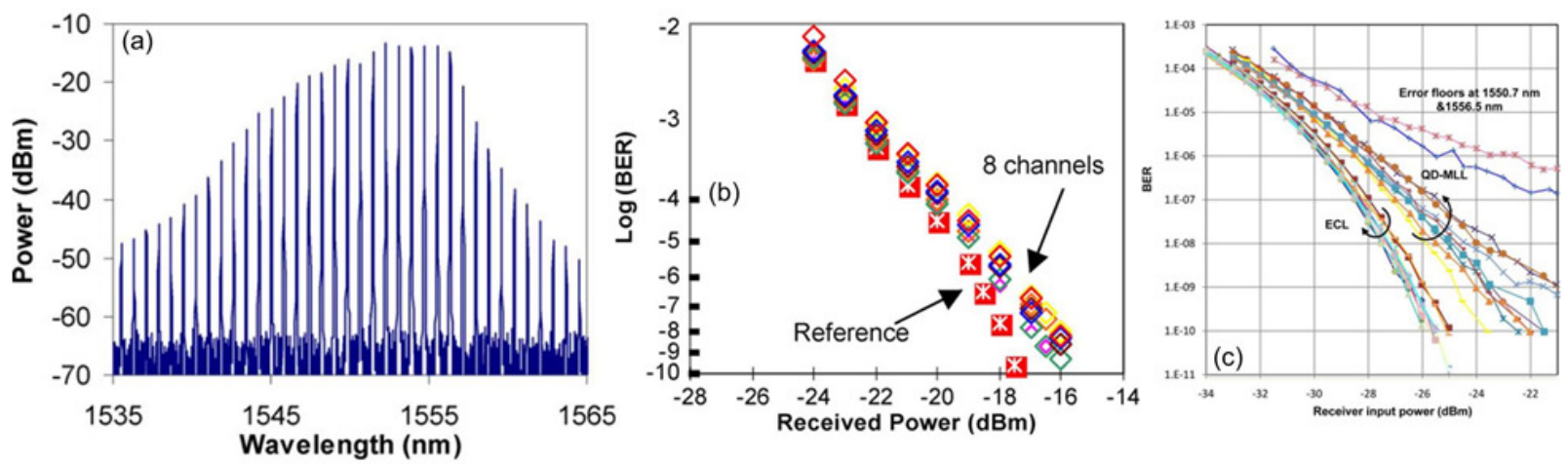

Fig. 37. (a) Optical spectrum of a $100 \mathrm{GHz}$ Qdash mode-locked laser. (b) BER for the Qdash mode-locked laser channels and a reference laser operating at $10 \mathrm{Gbps}$ on-off-keying modulation, and (c) BER versus receiver input power for 11 Qdash mode-locked laser channels, and a reference external cavity laser (ECL) operating at $10 \mathrm{Gbps}$ utilizing the DQPSK modulation scheme. Courtesy of [249, 251].

In addition, InAs/InGaAsP Qdash mode-locked lasers were also employed for wavelength tunability applications. For instance, Girault et al. [255] demonstrated a wavelength tunable RZ transmitter by exploiting the flat and wide band lasing emission $(\sim 19 \mathrm{~nm}-3 \mathrm{~dB}$ bandwidth) from Qdash lasers centered at $1.55 \mu \mathrm{m}$. The wavelength tuning was achieved by selecting a part of the lasing emission using an external optical filter. The potential of the Qdash mode-locked laser technology for the realization of a wavelength tunable transmitter for bit rates of $170 \mathrm{Gbps}$ [256] and $4 \times 170 \mathrm{Gbps}$ [257] were reported by Silva et al. In the former report [256], by adjusting the shaping filter frequency, tunability was achieved while in the latter case [257], four channels transmission was tested using the RZ pattern scheme, and an error floor for BER sensitivity of $10^{-8}$ up to $100 \mathrm{~km}$, and a penalty of $1 \mathrm{~dB}$ at BER $10^{-9}$ for back-to-back transmission, was demonstrated [258].

\subsubsection{Broadband Qdash lasers}

This new class of semiconductor laser diode source was first demonstrated by our group via exploiting the highly inhomogeneous nature of multi-stack InAs/InGaAlAs Qdash active region. A lasing bandwidth of $\sim 22 \mathrm{~nm}$ centered at $\sim 1.64 \mu \mathrm{m}$ was reported with a lasing emission coverage spanning $76 \mathrm{~nm}$ [259], as shown in Fig. 38(a). The 50×600 $\mu \mathrm{m}^{2}$ device exhibited a pulsed threshold current density of $2.6 \mathrm{kA} / \mathrm{cm}^{2}\left(650 \mathrm{~A} / \mathrm{cm}^{2}\right.$ per layer), slope efficiency $0.165 \mathrm{~W} / \mathrm{A}$, and output power $>400 \mathrm{~mW}$. The lasing wavelength of the device was successfully blue-shifted by $100 \mathrm{~nm}$ by postgrowth bandgap tuning utilizing the IFVD intermixing process. A widened lasing emission coverage of $85 \mathrm{~nm}$ with enhanced lasing bandwidth of $\sim 41 \mathrm{~nm}$ was reported by Tan et al. [260] from the $50 \times 500 \mu \mathrm{m}^{2}$ as-cleaved intermixed device which is also shown in Fig. 38(a). Furthermore, improved threshold current density of $2.1 \mathrm{kA} / \mathrm{cm}^{2}\left(525 \mathrm{~A} / \mathrm{cm}^{2}\right.$ per layer), slope efficiency $0.423 \mathrm{~W} / \mathrm{A}$, and characteristics temperature $\left(\mathrm{T}_{0} \sim 57 \mathrm{~K}\right.$ from 10 to $\left.60^{\circ} \mathrm{C}\right)$ were achieved. The total output power was $\sim 1 \mathrm{~W}$ which could potentially be employed as a highly efficient resonant pumping source for eyesafe Erbium-doped amplifiers and solid state lasers, besides applications in optical telecommunication, sensing and spectroscopy, optical metrology, biomedical imaging, etc. [261], as discussed in section 1. In view of extending the lasing bandwidth, we realized chirped barrier multistack Qdash active region (discussed in section. 4.4.) and reported a record $\sim 50 \mathrm{~nm}$ lasing bandwidth and lasing emission coverage of $\sim 65 \mathrm{~nm}$ from a $2 \times 830 \mu \mathrm{m}^{2}$ ridge-waveguide laser, illustrated in Fig. 38 (b). The device exhibited $3.6 \mathrm{kA} / \mathrm{cm}^{2}$ (900 A/ $\mathrm{cm}^{2}$ per layer) threshold current density, slope efficiency $0.36 \mathrm{~W} / \mathrm{A}$, and output power $>180 \mathrm{~mW}$ [8]. Moreover, we also studied the device physics of the chirped Qdash active region and found that the broad emission could be ascribed to the simultaneous emission for dispersive Qdashes [225] at high injection and the non-uniform distribution of carriers in the Qdash active region [262]. Intrinsic dynamics characteristics of these ultra-broadband Qdash lasers were also performed by Chen et al. [263]. A modulation efficiency of $\sim 0.3-0.6 \mathrm{GHz} / \mathrm{mA}^{0.5}$, and small signal modulation bandwidth of $\sim 3-6 \mathrm{GHz}$ was reported in the temperature range of $-40{ }^{\circ} \mathrm{C}$ to $40^{\circ} \mathrm{C}$. 

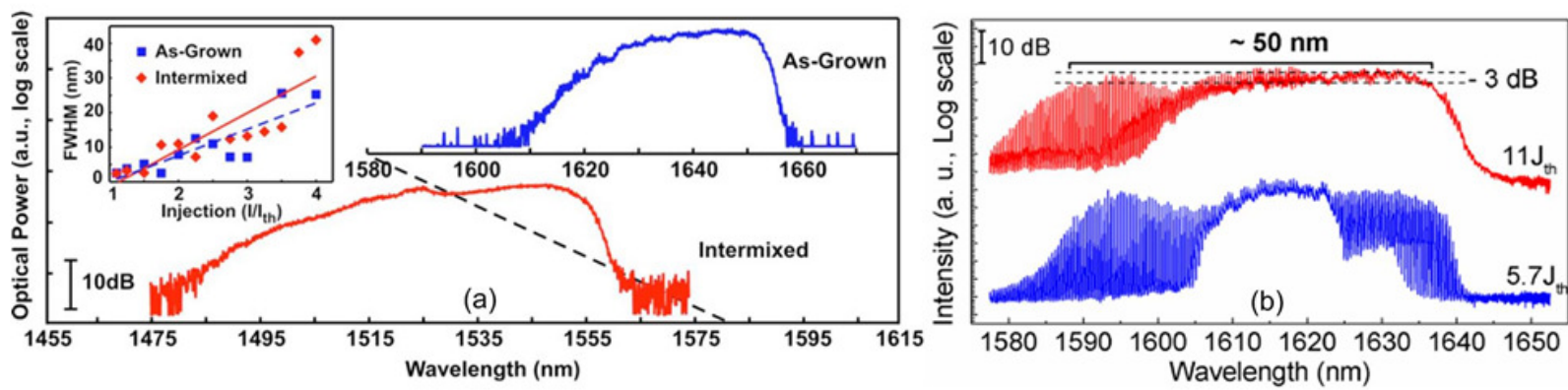

Fig. 38. (a) The wavelength tuned broadband fixed barrier 4 stack InAs/InGaAlAs DaWELL laser from $1.64 \mu \mathrm{m}$ (as-grown) to $1.54 \mu \mathrm{m}$ (IFVD intermixed) center wave length. The lasing coverage increases from 76 to $85 \mathrm{~nm}$ after the intermixing process. The inset shows the $-3 \mathrm{~dB}$ bandwidth of the broadband Qdash laser in accordance to injection. (b) Room temperature lasing spectra of $2 \times 830 \mu \mathrm{m}^{2}$ chirped barrier thickness 4 stack InAs/InGaAlAs DaWELL laser at different pulsed injection current density. A lasing bandwidth of $\sim 50 \mathrm{~nm}$ was measured. Taken from [8, 260].

To show the viability of this new class of lasers as a single multiwavelength source in WDM system, the broadband lasing spectrum was coupled into a 40-channel array waveguide gratings (AWG), and the lasing emission from each channel was measured [264]. A channel spacing of 100 $\mathrm{GHz}$ came from the AWG design rather from the broadband laser cavity length, with each channel exhibiting a $-3 \mathrm{~dB}$ bandwidth of $\sim 0.4 \mathrm{~nm}$. In addition, by varying the AWG temperature, a red-shift in the optical emission from a single AWG channel with a rate of $\sim 11.1 \mathrm{~A}^{\circ} /{ }^{\circ} \mathrm{C}\left(10^{\circ} \mathrm{C}\right.$ to $\left.30^{\circ} \mathrm{C}\right)$ was recorded thus showing the nearly ideal analogous wavelength tuning using a single broadband laser in conjunction with AWG. On the other hand, the discrete lon gitudinal modes of a FP cavity of the ultra-broadband Qdash lasers [8] wherein the mode spacing is a function of cavity length could also be a potential multi-wavelen gth source as discussed in section. 5.1.2.

\subsubsection{Single mode lasers}

A thorough investigation on the potential of the Qdash material platform has been carried out by fabricating, and testing single mode lasers and their capabilities. Here we reviewed the reported single mode laser on both the material platform and the achieved dynamic performances.
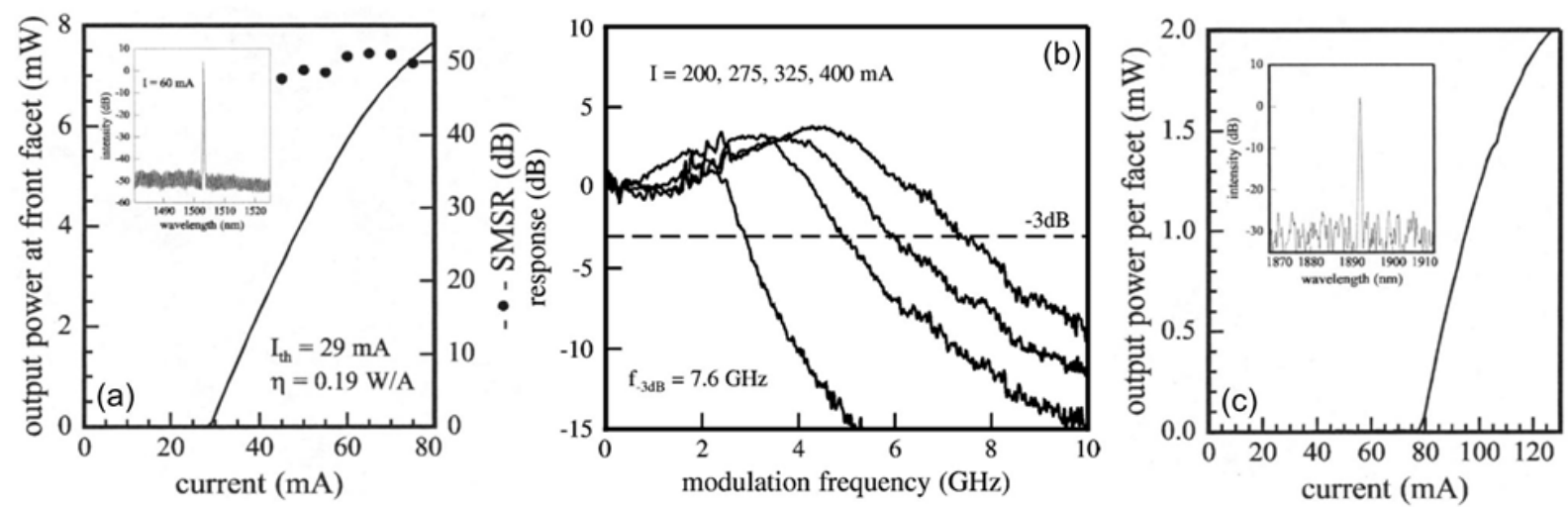

Fig. 39. (a) Output power and SMSR as a function of the drive current of a $600 \mu \mathrm{m}$ long InAs/InGaAlAs Qdash DFB laser under CW operation at room temperature. (b) Small signal modulation response of a $3 \times 1000 \mu \mathrm{m}^{2}$ Qdash DFB laser at four different pulsed current values. (c) Light output characteristic and emission spectra of a $1200 \mu \mathrm{m}$ long InAs DaWELL DFB laser emitting near $1.9 \mu \mathrm{m}$ at room temperature. Collected from [5, 265]

\section{In As/InGaAlAs Material System}

Bach et al. [266] initially reported a single mode Qdash laser based on distributed Bragg reflector (DBR) scheme and lasing at $1.54 \mu \mathrm{m}$. The 4-stack graded SCH Qdash laser with $300 \mu \mathrm{m}$ long grating un-pumped section and $2.5 \times 800 \mu \mathrm{m}^{2}$ gain section showed lasing at $210 \mathrm{~mA}$ with output power $>30 \mathrm{~mW}$ and SMSR $>40 \mathrm{~dB}$. From the temperature dependent measurements, a small shift in the lasing wavelength of $0.1 \mathrm{~nm} / \mathrm{K}\left(20\right.$ to $\left.70^{\circ} \mathrm{C}\right)$ was obtained and has been attributed to the temperature dependence of the active region refractive index. This work was soon followed by 
Kaiser et al. [267] who reported the dynamic properties of laterally coupled DFB gratings based 4 stack InAs/In GaAlAs Qdash laser, as shown in Fig. 39(a). A small signal modulation bandwidth of 7.6 GHz was measured from $3 \times 1000 \mu \mathrm{m}^{2}$ device under pulsed and CW operations, respectively (see Fig. 39(b)). A slope efficiency of $0.13 \mathrm{~W} / \mathrm{A}$, output power $>30 \mathrm{~mW}, 65 \mathrm{~mA}$ threshold current under $\mathrm{CW}$ operation, and a single peak at $1.51 \mu \mathrm{m}$ with SMSR of $>40 \mathrm{~dB}$ was observed. Under pulsed operation, the output power exceeded $110 \mathrm{~mW}$. By employing a deeply etched vertical grating DFB laser using Qdash active medium, Mathwig et al. [268] was able to push the modulation bandwidth up to $5.5 \mathrm{GHz}$ in $\mathrm{CW}$ operation. The fabricated 4 stack InAs/InGaAlAs graded $\mathrm{SCH}$ devices exhibited a threshold current of $24 \mathrm{~mA}$, SMSR > $48 \mathrm{~dB}$, and a wavelength shift of $0.1 \mathrm{~nm} / \mathrm{K}$. The tunability of Qdash DFB laser in the wavelength range of 1.5 to $1.9 \mu \mathrm{m}$ was shown by Kaiser et al. [265]. By employing InGaAs well based DaWELL or graded SCH InAs/InGaAlAs Qdash structure, a single mode lasing at $1.9 \mu \mathrm{m}$ and $1.51 \mu \mathrm{m}$, respectively, with corresponding SMSR of $>25 \mathrm{~dB}$ and $>45 \mathrm{~dB}$ were demonstrated, as depicted in Fig. 39(c). In addition, the short wavelength devices showed a slope efficiency of $0.06 \mathrm{~W} / \mathrm{A}, 76 \mathrm{~mA}$ threshold current, and output power $>2.0 \mathrm{~mW}$; while the long wavelength devices showed a slope efficiency of 0.19 W/A, $29 \mathrm{~mA}$ threshold current, and output powers $>80 \mathrm{~mW}$ [26]. Subsequently, the comparatively inferior performance of the long wavelength InAs Qdash in InGaAs Qwell DFB lasers was improved by Zeller et al. [269] and Hein et al. [270] who reported single mode lasers at $2.01 \mu \mathrm{m}$ and $1.89 \mu \mathrm{m}$, respectively, at room temperature $\mathrm{CW}$ operation. In the former case, a threshold current of $40 \mathrm{~mA}$ from $2 \times 900 \mu \mathrm{m}^{2}$ device showed a high SMSR of $>35 \mathrm{~dB}$ with good reliability properties (maintained CW output power at 60 $\mathrm{mA}$ up to $4800 \mathrm{~h}$ ). The latter case demonstrated a slope efficiency $0.22 \mathrm{~W} / \mathrm{A}$ with output power $>25$ $\mathrm{mW}$, and SMSR > $35 \mathrm{~dB}$ and temperature coefficient $0.14 \mathrm{~nm} / \mathrm{K}$.
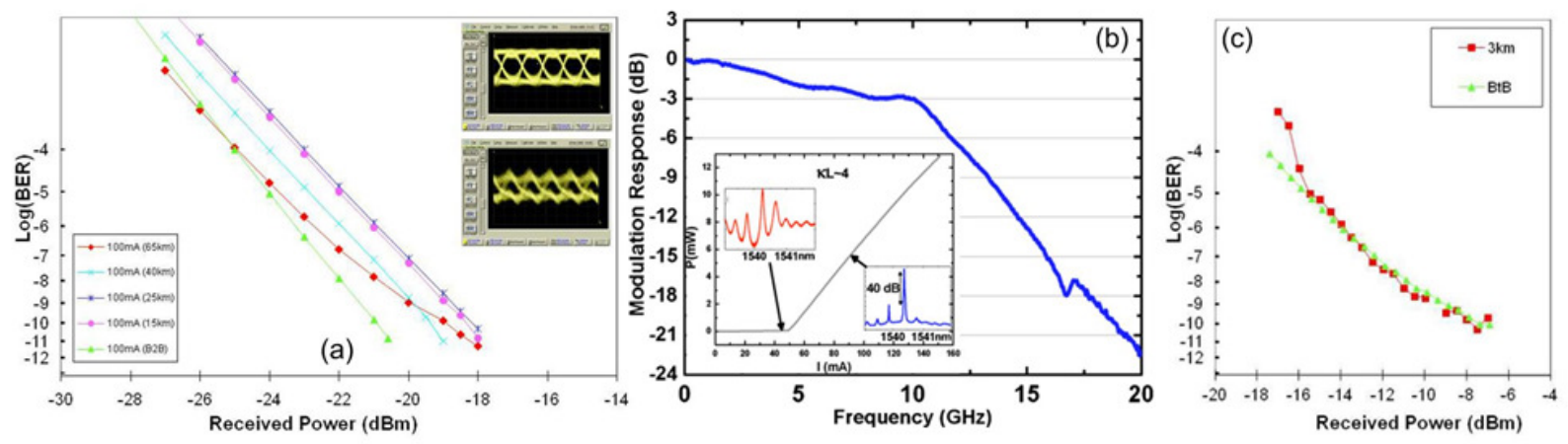

Fig. 40. (a) $10 \mathrm{Gbps} \mathrm{BER}$ at $25^{\circ} \mathrm{C}$ in back-to-back and after $15,25,40$ and $65 \mathrm{~km}$ single mode fiber transmission. Inset in (a) shows the eye diagrams in back-to-back and after $65 \mathrm{~km}$ transmissions. (b) Small signal modulation response of $1000 \mu \mathrm{m}$ long p-doped buried ridge-waveguide InAs/InGaAsP Qdash DFB laser at $150 \mathrm{~mA}$ injection current. Insets in (b) show the corresponding $L-I$ characteristic and optical spectrum at $46 \mathrm{~mA}$ (left) and $90 \mathrm{~mA}$ (right). (c) $20 \mathrm{Gbps} \mathrm{BER}$ at $25^{\circ} \mathrm{C}$ in back-to-back and after single mode fiber transmission over $3 \mathrm{~km}$, of a $500 \mu \mathrm{m}$ undoped and optimized active region Qdash DFB laser. Reproduced from [239, 271, 272].

\section{$\underline{\text { In As/InGaAsP Material System }}$}

A single mode Qdash laser on this material system was demonstrated by Dagens et al. [273], reporting a $10 \mathrm{Gbps}$ direct large signal modulation with a buried ridge-waveguide DFB laser operating at $1.51 \mu \mathrm{m}$. The $0.8 \times 205 \mu \mathrm{m}^{2}$ device was based on 6-stack DaWELL structure grown by MBE-MOCVD techniques, exhibiting $4.8 \mathrm{~mA}$ threshold current, $0.3 \mathrm{~W} / \mathrm{A}$ slope efficiency, and SMSR about $45.5 \mathrm{~dB}$. The small signal modulation bandwidth reached around $6.7 \mathrm{GHz}$ with high modulation efficiency of $1.9 \mathrm{GHz} / \mathrm{mA}^{0.5}$ and K-factor $0.43 \mathrm{~ns}$ [207]. Furthermore, the device chirp was quantified via above-threshold LEF with measured value of 4.5-6.5. The BER measurements of this direct modulation laser before and after transmission revealed a floor free measurement with $10^{-}$ ${ }^{10}$ sensitivity of $-13 \mathrm{dBm}$. In spite of large LEF, after transmission through 2,8 , and $10 \mathrm{~km}$ stand ard fibers (metropolitan distance), floor-free transmission was achieved with $0.8,4$, and $5 \mathrm{~dB}$ penalty, respectively. Following this work, utilizing optimized DaWELL active region design and by reducing the Qwell thickness from $8 \mathrm{~nm}$ to $6 \mathrm{~nm}$, Dagens et al. [274] reported a $1.0 \times 240 \mu \mathrm{m}^{2} \mathrm{DFB}$ 
laser with CW threshold current around $4.4 \mathrm{~mA}$, lasing wavelength $\sim 1.55 \mu \mathrm{m}$ with SMSR $33 \mathrm{~dB}$. The device showed a high modulation efficiency of $1.6 \mathrm{GHz} / \mathrm{mA}^{0.5}$ and $\mathrm{K}$-factor $0.3 \mathrm{~ns}$. The large signal modulation of $10 \mathrm{Gbps}$ NRZ PRBS signal on this laser at $35^{\circ} \mathrm{C}$ and $85^{\circ} \mathrm{C}$ demonstrated floorfree BER at $10^{-10}$ and $-9 \mathrm{dBm}$ with no penalty both in back-to-back and after $10 \mathrm{~km}$ transmission. By reducing the $\mathrm{SCH}$ layer thickness in addition to the above optimized DaWELL laser structure, Lelarge et al. [239] further investigated $0.5 \mathrm{~mm}$ and $1.0 \mathrm{~mm}$ buried ridge-waveguide DFB lasers at $1.54 \mu \mathrm{m}$. The SMSR of $\sim 40 \mathrm{~dB}$ was obtained with threshold currents around $30 \mathrm{~mA}$ and output power $>10 \mathrm{~mW}$. Direct small signal modulation bandwidth of $\sim 10 \mathrm{GHz}$ was measured with K-factor as low as $0.3 \mathrm{~ns}$ and reduced above threshold LEF $<2$. As shown in Fig. 40(a), an error free $10 \mathrm{Gbps}$ transmission was reported up to $65 \mathrm{~km}$ with BER sensitivity $10^{-10}$ at -18 to $-20 \mathrm{dBm}$ received power, and $3 \mathrm{~dB}$ extinction ratio.

Active region p-doped InAs/InGaAsP Qdash buried ridge-waveguide index coupled DFB laser was also demonstrated in the material system by Zou et al. [271]. The $1 \mathrm{~mm}$ long device exhibited a threshold current of $50 \mathrm{~mA}$, slope efficiency $0.26 \mathrm{~W} / \mathrm{A}$ at $1.54 \mu \mathrm{m}$ with $40 \mathrm{~dB}$ SMSR. A small signal modulation bandwidth of $>10 \mathrm{GHz}$ was measured from the device at $150 \mathrm{~mA} \mathrm{CW}$ bias current and is plotted in Fig. 40(b). The corresponding modulation efficiency and above-threshold LEF values were $1.1 \mathrm{GHz} / \mathrm{mA}^{0.5}$ and $<2$, respectively [275]. These performance characteristics were further improved very recently by Chimot et al. [272] who reported large signal modulation of 20 Gbps NRZ PRBS error free transmission up to $3 \mathrm{~km}$ with BER sensitivity of $10^{-10}$ at $-10 \mathrm{dBm}$ received power and $>10 \mathrm{GHz}$ small signal modulation bandwidth, from the $500 \mu \mathrm{m}$ buried ridgewaveguide DFB laser at $1.54 \mu \mathrm{m}$ (see Fig.40(c)). Besides, the extinction ratio of $3 \mathrm{~dB}$ was improved to 6-8 $\mathrm{dB}$ by combining the Qdash laser with a commercially av ailable elaton filter.

\subsubsection{Injection locking/critical feedback of Qdash lasers}

The dynamic properties of InAs/InP Qdash lasers were further improved by optical injection under stable locking conditions by Li et al. [276] who showed a three times increase in the small signal modulation bandwidth of the laser. The $4 \times 500 \mu \mathrm{m}^{2}$ injection locked device exhibited a $-3 \mathrm{~dB}$ bandwidth of $8.7 \mathrm{GHz}$ at $-8.6 \mathrm{dBm}$ injected power under zero detuning compared to $3.4 \mathrm{GHz}$ for the free-running case. In another study, a value of $11.7 \mathrm{GHz}$ was observed at $3.5 \mathrm{dBm}$ injected power in the master laser by Naderi et al. [277] under stable locking conditions with $30 \mathrm{~dB}$ SMSR. This corresponded to a threefold improvement relative to the free-running case. The LEF was also measured above threshold and varied from 1 at threshold to 11 at $90 \mathrm{~mA}$, attributed to the carrier density being unclamped at threshold which is due to the inhomogeneous gain broadening in Qdashes. Recently, an impressive $16.5 \mathrm{GHz}-3 \mathrm{~dB}$ small signal modulation bandwidth was reported by Lester et al. [278] by slightly blue shifted $(1.535 \mu \mathrm{m})$ injection locked Qdash laser under strong optical injection of $9.3 \mathrm{~dB}$. This was approximately four times bandwidth enhancement compared to the free-running $4.5 \mathrm{GHz}$ at $1.565 \mu \mathrm{m}$. In addition, remarkable values of near zero above-threshold LEF and $5.9 \times 10^{-14} \mathrm{~cm}^{-2}$ differential gain were obtained under these conditions. This is highly attractive as packaged RF photonic transmitter in high-frequency optical fiber links [278, 279]. A detailed dynamic study of an injection locked Qdash laser was carried out by Pochet et al. [280, 281] at zero detuning, and it was found that Qdash laser's large damping rate, gain compression coefficient, and sufficiently small LEF yielded period-one and stable locking operating conditions at bias curren ts close to threshold. In fact, under period-one state, a tunable photonic oscillator based on optical-injection Qdash laser was demonstrated by varying the injection field ratio from $4.9 \mathrm{GHz}$ to $45.4 \mathrm{GHz}$ [282]. A tunable reson ance frequency from $4.9 \mathrm{GHz}$ to $8.3 \mathrm{GHz}$ was reported.

Till now, the beneficial effects of the optical feedback were discussed wherein a very weak or a very strong optical injection was employed to achieve improved performance characteristics of Qdash lasers. However, optical feedback on the other hand results in laser instability causing collapse of the coherence time and hence broadening of the lasing spectrum, in addition to degradation in the LEF as well as the BER. The tolerance to the optical feedback of Qdash lasers was first studied by Azouigui et al. [234] who demon strated an onset of coherence collapse from -41 to $-27 \mathrm{~dB}$ on increasing the current injection of $205 \mu \mathrm{m}$ long InAs/InGaAsP Qdash DFB laser from 10 
to $100 \mathrm{~mA}$, based on optical spectrum and RIN static characteristics. In terms of dynamic characteristics at $10 \mathrm{Gbps}$ PRBS transmission signal operating at $30 \mathrm{~mA}$, a floor-free operation (BER sensitivity $10^{-10}$ at $\sim-13.5 \mathrm{dBm}$ ) was achieved at $-32 \mathrm{~dB}$ (correspond to $-24 \mathrm{~dB}$ return loss and $\sim 4.5$ LEF ) and the performance degraded above this value. The onset of coherence collapse was further improved on a $600 \mu \mathrm{m}$ FP Qdash laser with values ranging from -29 to $-21 \mathrm{~dB}$ (as a function of current) and hence $-19 \mathrm{~dB}$ return loss, thus complying with the requirement of IEEE $802.3 \mathrm{ae} 10$ Gbps Ethernet standard [235]. A systematic investigation of the external feedback effect on several Qdash lasers exhibiting different LEF values ( 3.6 to $\sim 10.7)$ and damping factors ( 9 to $\sim 5.2)$ were also performed. An onset of coherence collapse as good as $>-18 \mathrm{~dB}$ was achieved with linear dependence on the drive current, and thus highlighted the better performance from high differential gain devices [283]. In addition, the effect of temperature and cavity length was also investigated by Azouigui et al. [284] and it was found that higher optical feedback could be achieved from temperature insensitive differential gain laser and/or longer cavity devices. In general, these features were similar to the bulk or Qwell lasers but with improved critical feedback level, and both InAs/InP Qdots and Qdash lasers with similar differential gain and cavity length exhibited similar onset of coherence collapse. In contrast, Grillot et al. [285] found a decrease in the critical feedback level with increasing current injection from a $500 \mu \mathrm{m}$ long ridge-waveguide Qdash laser; an unconventional trend compared to the Qwell lasers. This was attributed to the contributions of the ES coupled to the non-linear effects which caused non-linear increase in the GS above-threshold LEF from $\sim 1$ to $\sim 14$ on increasing current injection, thus emerging as a key parameter for designing feedback-resistant lasers [286].

\subsection{InAs/InP Qdash mode-locked lasers}

The broad stimulated spectrum width of the InAs/InP Qdash lasers showing sub-picosecond pusle generation capability has also been the centre of attraction since a decade and demonstrated impressive results in terms of pulse width, repetition frequency and particularly RF spectrum bandwwidth which was observed to be from $<1 \mathrm{kHz}$ up to $600 \mathrm{kHz}$. However, in general, reduction of dimenstionality from zero dimensional Qdots to quasi-zero dimentional Qdashes has no significant impact on the mode locking characteritics of InAs/InP nanostructure lasers. This observation has been deduced based on our discussion on In As/InP Qdots mode-locked lasers (section. 3.2) and comparison with the achievments in InAs/InP Qdash mode-locked lasers summarized in this section and plotted in Fig. 2(b).

\subsubsection{Two-section}

Passive mode locking employing classical two-section In As/InGaAsP Qdash laser with one gain and one absorber section was first demonstrated by Gosset et al. [287] who measured $47 \mathrm{kHz} \mathrm{RF}$ linewidth from a 6-stack Qdash laser with $940 \mu \mathrm{m}$ gain and $180 \mu \mathrm{m}$ absorber sections. A pulsation frequency of $43.6 \mathrm{GHz}$ was measured at $169 \mathrm{~mA} \mathrm{CW}$ current and with no significant effect of applied bias on the absorber section. This was attributed to the FWM phenomenon in the gain section to be dominant compared to the effect of the absorber section. Later, Merghem et al. [288] investigated a $2.4 \mathrm{~mm}$ Qdash mode-locked laser with absorber/gain length ratio of $4 \%$ which corresponds to a repetition frequency of $17 \mathrm{GHz}$ at $1.58 \mu \mathrm{m}$. Pulse widths ranging from 3 to $14 \mathrm{ps}$ (after deconvolution), and RF linewidth values from $250 \mathrm{kHz}$ to $3.5 \mathrm{MHz}$ were observed for a wide operating regime (current injection from 60 to $160 \mathrm{~mA}$ and reverse bias voltage from -5 to $0 \mathrm{~V}$ ). It was shown that when the mode-locked laser was subjected to optical feedback at $\sim-22 \mathrm{~dB}$, the mode-beating linewidth substantially narrowed to $<1.0 \mathrm{kHz}$ to $500 \mathrm{kHz}$ range with slight increase in the pulse duration with a smallest value of $\sim 500 \mathrm{~Hz}$ at current values 100 to $130 \mathrm{~mA}$ compared to $370 \mathrm{kHz}$ without the optical feedback. Later, Dontabactouny et al. [289] studied the phase noise and timing jitter on a $10 \mathrm{GHz}$ two section $3.95 \mathrm{~mm}(0.13 \mathrm{~mm}$ absorber section) long 5 stacks InAs/In GaAsP Qdash laser emitting at $1.59 \mu \mathrm{m}$. Highly chirped pulses ranging from 8 to 14 ps were observed at a fixed bias current and reverse bias voltage and by changing the position of a $1 \mathrm{~nm}$ 
bandwidth filter centered at $1.6 \mu \mathrm{m}$. By employing $545 \mathrm{~m}$ single mode optical fiber for dispersion compensation, pulses in picosecond range were reported with the smallest value 975 fs after deconvolution. Phase noise measurements revealed values $-80 \mathrm{dBc} / \mathrm{Hz}$ at $100 \mathrm{kHz}$, which reduced down to $-140 \mathrm{dBc} / \mathrm{Hz}$ at $100 \mathrm{MHz}$, while an average timing jitter as low as $800 \mathrm{fs}$ was reported. Similar mode locking characteristics on $1.0 \mathrm{~mm}$ device (4\% absorber length) corresponding to 41 $\mathrm{GHz}$ pulse repetition rate was also investigated which again exhibited strongly chirped pulses. Pulse duration of $\sim 18 \mathrm{ps}$ (raw pulses) with $\sim 1 \mathrm{MHz}$ RF linewidth was observed and when passed through a $60 \mathrm{~m}$ single mode fiber decreased to $\sim 7 \mathrm{ps}$ with $\sim 100 \mathrm{kHz}$ mode-beating linewidth.

A systematic passive mode locking investigation at repetition rates of 20,48 and $95 \mathrm{GHz}$ from $1200 \mu \mathrm{m}$ (long), $890 \mu \mathrm{m}$ (medium) and $450 \mu \mathrm{m}$ (short) length (10\% absorber section) In As/InGaAsP Qdash lasers were reported by Rosales et al. [250, 290, 291] with $95 \mathrm{GHz}$ repetition value being the highest value ever reported on a monolithic two-section device in any InAs/InP Qdash material system. The results are summarized in Figs. 41(a)-(c). Stable mode locking regime in all three lasers were discussed in detail with minimum achievable pulse durations $\sim 1.4, \sim 2.5$ and $\sim 1.6$ ps without deconvolution, and time bandwidth product of 1.7, 1.5 and 1.4, for the long, medium, and short cavity lasers, respectively. These values were obtained for low drive currents at a reasonably high reverse bias value. Noise trends were also assessed by RF linewidth measurement showing small values of $\sim 100 \mathrm{kHz}$ from the medium cavity laser and $\sim 20 \mathrm{kHz}$ from the long and short cavity lasers. Measurements of group delay (GD) and group delay dispersion (GDD) were also performed by Rosales et al. [292] on a $48 \mathrm{GHz}, 890 \mu \mathrm{m}$ long optimized Qdash laser with $60 \mu \mathrm{m}$ absorber section. Deconvolved pulses down to 1.2 ps were observed after compensation of the GDD and 1.7 time bandwidth product. Similar results were also achieved from two-section InAs/In GaAlAs Qdash laser by Lin et al. [293] who demonstrated 12.3 and $18.4 \mathrm{GHz}$ repetition rates from 3.4 and $2.3 \mathrm{~mm}$ long devices, respectively, emitting at $1.59 \mu \mathrm{m}$. In general, lower average output powers due to low drive current besides the inability to attain wider spectral bandwidth render the two-section mode-locked laser application s compared to the single section device

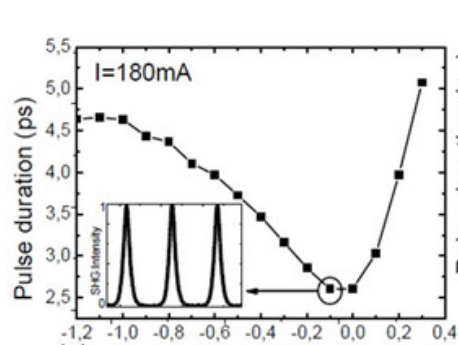

(a)
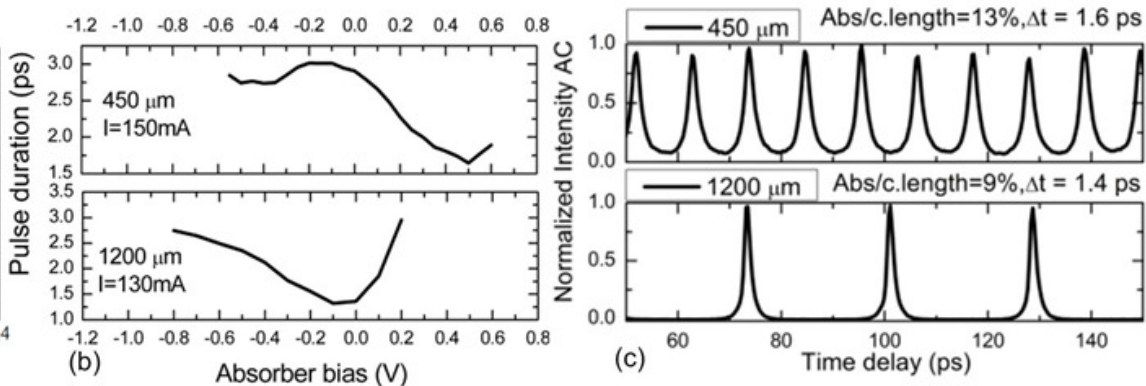

Fig. 41. (a) Pulse duration as a function of reverse bias at a fixed drive current of $180 \mathrm{~mA}$ for $1.5 \times 890 \mu \mathrm{m}^{2} \mathrm{InAs} / \mathrm{InGaAsP}$ Qdash mode-locked laser with $90 \mu \mathrm{m}$ absorber section and yielding a repetition rate of $48 \mathrm{GHz}$ (shown in the inset). (b) The effect of reverse bias on the pulse width for the 450 $\mu \mathrm{m}$ and $1200 \mu \mathrm{m}$ long high ga in InAs/InGaAsP Qdash mode-locked laser and (c) the corresponding autocorre lation pulse train. Taken from [290, 291].

\subsubsection{Self-pulsation}

Single section passive mode locking is highly attractive compared to the two-section mode locking in terms of attainable output power and pulse width, and has been actively researched since a decade with superior performances. As discussed in section 3.2.2, a $45 \mathrm{GHz} \mathrm{CW}$ mode locking from monosection InAs/InP Qdot/Qdash laser was first demonstrated by Renaudier et al. [120]. Following this report was the detailed investigation by Gosset et al. [287] who demonstrated $134 \mathrm{GHz}$ pulse generation from $340 \mu \mathrm{m}$ long 6-stack InAs/InGaAsP DaWELL laser under CW operation. The 1.56 $\mu \mathrm{m}$ device exhibited deconvolved sub-picosecond pulse width of 800 fs without any pulse compression scheme and 0.46 time bandwidth product. RF spectral linewidth of $50 \mathrm{kHz}$ was measured from the $990 \mu \mathrm{m}$ device exhibiting $42.2 \mathrm{GHz}$ pulse repetition rate and $2 \mathrm{ps}$ auto-correlated pulses. The ability to reduce the mode beating linewid th to $2 \mathrm{kHz}$ by an inclusion of an optical filter was demonstrated by Shen et al. [294] on a $2.5 \mathrm{~mm}$ long, $17 \mathrm{GHz}$ Qdash mode-locked laser, and 
further showed that reduction in the optical confinement factor also decreased the mode beating linewidth [295]. In general, these high performance characteristics were attributed to the reduction in the active region dimensionality resulting in reduced interaction of the optical mode with the amplified spontaneous emission, and large population inversion, which reduces the phase noise, apart from FWM process [250, 296]. On the other hand, Akrout et al. [297] exploited the external cavity length based high quality factor for initial phase noise reduction in a $30.3 \mathrm{GHz}$ In As/InGaAsP/InP Qdash self-pulsating mode-locked laser. The auto-optical feedback loop resulted in the reduction of the mode-beating linewidth (phase noise) from $30 \mathrm{kHz}(-75 \mathrm{dBc} / \mathrm{Hz})$ to mere 200 $\mathrm{Hz}(-105 \mathrm{dBc} / \mathrm{Hz})$.

A systematic investigation of timing jitter on 9-stack InAs/InGaAsP Qdash-in-barrier laser was performed by Tourrenc et al. [298] on a $40 \mathrm{GHz}$ mode-locked laser exhibiting 20 dB extinction ratio, $3.71 \mathrm{ps}$ deconvolved pulse wid th and $240 \mathrm{kHz} \mathrm{RF}$ linewidth. A value of $860 \mathrm{fs}$ was reported in the $1 \mathrm{MHz}$ to $20 \mathrm{MHz}$ range and an optimized RF linewidth of $30 \mathrm{kHz}$ was also achieved at $242 \mathrm{~mA}$ with an estimated reduction in the timing jitter to $280 \mathrm{fs}$. These performance parameters were further improved by Latkowski et al. [299] who successfully measured 720 fs pulse width from a $39.8 \mathrm{GHz}$ Qdash mode-locked laser by passing through a $450 \mathrm{~m}$ single mode dispersion compensated fiber. Furthermore, as depicted in Fig. 42(a), mode beating linewidth of 10 to $25 \mathrm{kHz}$ was measured regardless of the bias current value and the timing jitter varied from $350 \mathrm{fs}$ to $150 \mathrm{fs}$ with peak power varying from $40 \mathrm{~mW}$ to $140 \mathrm{~mW}[300,301]$. The influence of the bias current and the filter bandwidth on the pulses were also performed which revealed decrease in the pulse width either by increasing filter bandwidth or the bias current. This was related to locking of larger number of longitudinal modes and a better respective phase locking at higher injection [302, 303]. Further improvement of the $40 \mathrm{GHz}$ mode-locked laser characteristics was achieved by Maldonado-Basilio et al. [304] who reported best ever $8 \mathrm{~Hz}$ RF linewidth and $64 \mathrm{fs}$ timing jitter by subjecting the laser to an optical injection by its fourth harmonic i.e. $10 \mathrm{GHz}$ pulses at $\sim 1.55 \mu \mathrm{m}$, as shown in Fig. 42(b). In terms of highest repetition rate, Merghem et al. [245] reported a record $346 \mathrm{GHz}$ pulse generation (see Fig. 42(c)) using a passively mode-locked $120 \mu \mathrm{m}$ long Qdash laser emitting 560 fs deconvolved pulses with $20 \mathrm{~mW}$ peak power and $9 \mathrm{~dB}$ extinction ratio. On the other hand, a $170 \mu \mathrm{m}$ 6 stacks Qdash mode-locked laser exhibited $870 \mathrm{fs}$ pulses at $245 \mathrm{GHz}$ with $100 \mathrm{~mW}$ peak power and $7 \mathrm{~dB}$ extinction ratio.
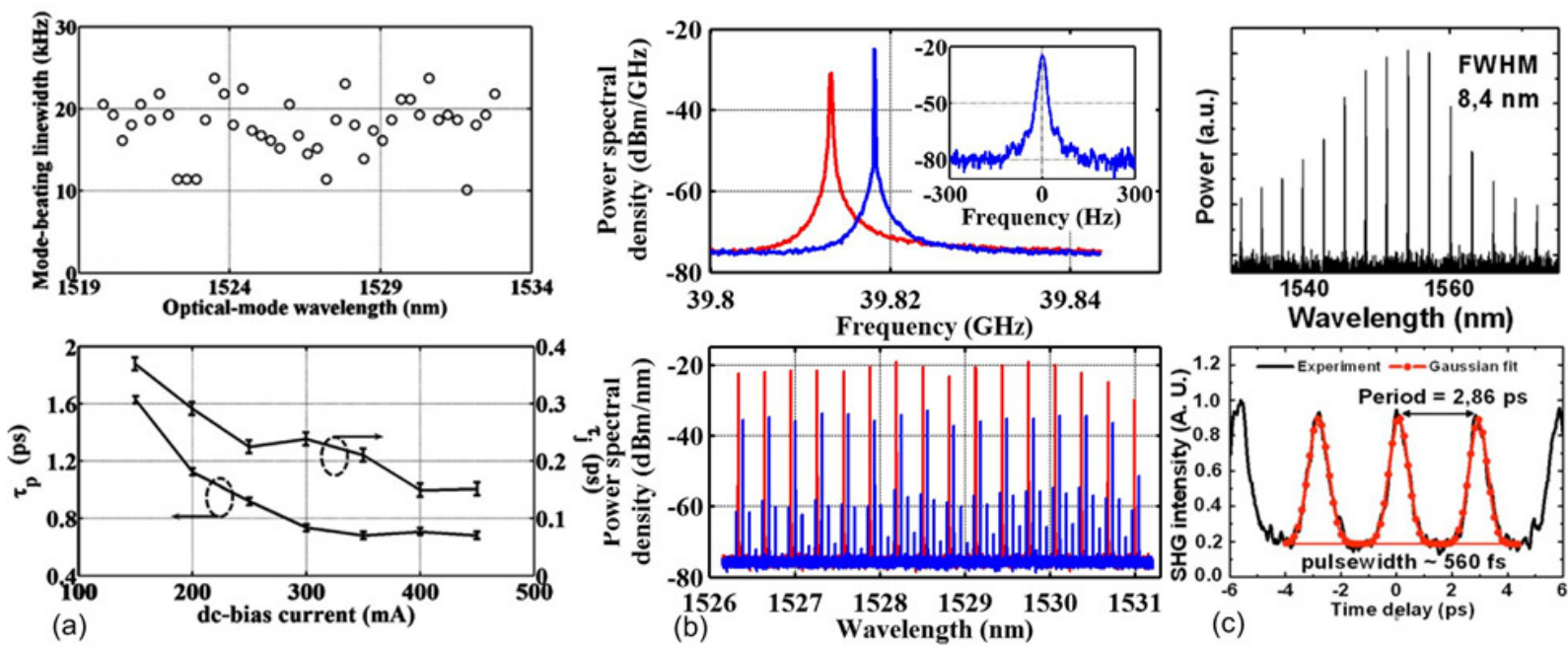

(c)

Fig. 42. (a) Typical mode-beating associated with each pair of optical modes as a function of the filter central wavelength (top) of the $1000 \mu \mathrm{m}$ long 40 $\mathrm{GHz}$ InAs/InGaAsP Qdash mode-locked laser at $350 \mathrm{~mA}$. The corresponding device pulse width and timing jitter as sociated with the optically generated pulses as a function of CW current (bottom). (b) Beat-tones (top) and optical spectrum (bottom) for the $1000 \mu \mathrm{m}$ long $40 \mathrm{GHz}$ InAs/InGaAsP Qdash mode-locked laser under free-running (red trace) and under the injection of $10 \mathrm{GHz}$ optical pulses (blue trace), at bias current of $100 \mathrm{~mA}$. Inset illustrates the beat-tone under optical injection rec orded with a $10 \mathrm{~Hz}$ resolution showing $8 \mathrm{~Hz}$ linewidth. (c) Optical spectrum (top) and autoc orrelation trace (bottom) of $120 \mu \mathrm{m}$ long, $345 \mathrm{GHz}$, InAs/InGaAsP Qdash mode-locked laser at $217 \mathrm{~mA}$. Courtesy of [245, 301, 304]

Instead of the typical stepped-heterodyne technique for pulse characterization, Calo et al. [305] employed the second-harmonic generation frequency resolved optical gating (SHG-FROG) technique for the investigation of high gain $\sim 50 \mathrm{~cm}^{-1}, 1.56 \mu \mathrm{m}, 9$-stack InAs/InGaAsP dash-in- 
barrier laser. The $890 \mu \mathrm{m}$ long device exhibited a repetition frequency of $\sim 48 \mathrm{GHz}$ under $\mathrm{CW}$ operation. Highly chirped pulses broadened by positive GDD were compressed by a single mode fiber to achieve $423 \mathrm{fs}$ pulses with $\sim 100 \mathrm{~mW}$ peak power at $300 \mathrm{~mA}$ bias [306]. The value was found to reduce at $400 \mathrm{~mA}$ injection reaching a $374 \mathrm{fs}$ which is the best value reported on an InAs/InP Qdash material system, and is shown in Fig. 43(a). By developing an asymmetrical cladding Qdash laser with extremely small loss, Faugeron et al. [247] reported small RF linewidth of $300 \mathrm{~Hz}$ from 4.4 GHz $10 \mathrm{~mm}$ long single section passive Qdash mode-locked laser. Furthermore, a record high peak power of $18 \mathrm{~W}$ was demonstrated from $4.3 \mathrm{~mm}$ long $10 \mathrm{GHz}$ mode-locked laser generating 750 fs deconvolved pulses after pulse compression via $570 \mathrm{~m}$ single mode fiber (compensating $9.3 \mathrm{ps} / \mathrm{nm}$ linear GD). An improved phase noise below $-100 \mathrm{~dB} / \mathrm{Hz}$ at an offset frequency of $1 \mathrm{kHz}$ and $\mathrm{RF}$ harmonics up to $120 \mathrm{GHz}$ was also demonstrated by active mode locking the $4.3 \mathrm{~mm}$ long Qdash laser via electrical $\sim 10 \mathrm{GHz}$ RF signal injection [211]. Very recently, an integration of 6 stacks In As/InGaAsP Qdash mode-locked laser in DBR configuration and with an integrated SOA was demonstrated by Joshi et al. [307]. By careful control of the DBR gratings an RF linewidth down to $30 \mathrm{kHz}$ was reported from the $40 \mathrm{GHz} 1.5 \times 1000 \mu \mathrm{m}^{2}$ device at a CW injection of $310 \mathrm{~mA}$. It was shown that the linewidth was maintained with and without biasing the SOA, except the regions of mode locking were changed. This is ascribed to the interaction of the laser emission with the amplified spontaneous emission of the SOA. A pulse width of 1.4 ps was obtained after chirp compensation with $120 \mathrm{~m}$ single mode fiber [308]. Besides, the capability of using SOA as modulator was also demonstrated by reporting a floor free BER sensitivity of $10^{-10}$ at $-34 \mathrm{dBm}$ via on-off keying modulation at 5 Gbps [309].

The phase correlation of the adjacent longitudinal modes in a passively mode-locked Qdash laser was investigated by Duan et al. [241, 310] by measuring the RF linewid th of couples of modes at three different wavelength regime selected by an optical filter. A fixed linewidth of $15 \mathrm{kHz}$ was obtained for all the three groups and was same as the spectral linewidth of the entire optical spectra, thus affirming that the relative phases of the adjacent modes have the same phase noise characteristics [207]. Alternatively, Rosales et al. [292, 311] and others [312-314] shed light on the spectral phase profile of the InAs/InGaAsP Qdash mode-locked lasers which determine the pulse shape. In the former report, employing a $\sim 48 \mathrm{GHz}(\sim 890 \mu \mathrm{m})$ and $23 \mathrm{GHz}(1820 \mu \mathrm{m}) \mathrm{InAs} / \mathrm{InGaAsP}$ Qdash mode-locked laser exhibiting corresponding minimum values of $20 \mathrm{kHz}$ and $100 \mathrm{kHz} \mathrm{RF}$ linewidth, GD and spectral phase of the electric field were determined and GDD values were calculated to be 1.3 and $3.0 \mathrm{ps}^{2}$, respectively. No pulse generation was observed without the dispersion compensation by single mode fibers. The shorter cavity yielded 600 fs pulses with $40 \mathrm{~mW}$ average power $(\sim 1 \mathrm{~W}$ peak power) emitting at $\sim 1.55 \mu \mathrm{m}$ while the longer cavity locked at $700 \mathrm{fs}$ pulses.
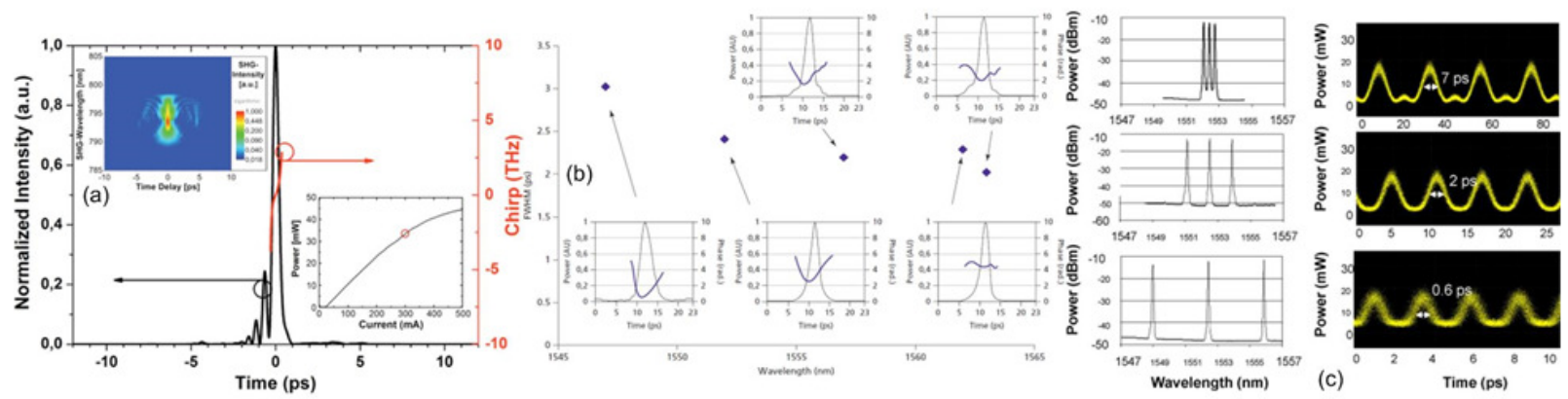

Fig. 43. (a) Intensity and chirp profile of pulse ge nerated by a $890 \mu \mathrm{m}$ long single-section Q dash mode-locked laser for an injection current of $300 \mathrm{~mA}$, after dispersion compensation using $66 \mathrm{~m}$ of single mode fiber. In the inse ts: (left) measured FROG spectrogram, (right) light-current characteristic of the laser. (b) Pulse width as a function of the tunable opticalfilter center wavelength for an actively mode-locked Qdash la ser. Pulse shape and chirp are shown in the inset. (c) Tunable pulse ge neration at three different frequencies: $42.7 \mathrm{GHz} ; 170.8 \mathrm{GHz}$, and $427 \mathrm{GHz}$, from a single $42.7 \mathrm{Qdash}$ actively mode-locked laser and a tunable spectral filter. Adapted from [241, 258, 306]

Tunable pulse generation utilizing Qdash active mode-locked laser via actively mode-locking through the electrical modulation at $42.7 \mathrm{GHz}$ was demonstrated by employing a tunable optical filter up to $16 \mathrm{~nm}$ over the laser emission spectrum with relatively fixed pulse width of $2.4 \pm 0.4 \mathrm{ps}$ 
and time bandwidth product $0.48 \pm 0.09$ at $42.7 \mathrm{GHz}$ [295, 310] as shown in Fig. 43(b). In addition, by inclusion of pulse compression approach via single mode fiber, a pulse width reduction down to 1.1 ps was obtained by Akrout et al. [315]. On the other hand, Costa-e-Silva et al. [256, 258] showed that a tunable pulse generation at $42.7 \mathrm{GHz}, 170.8 \mathrm{GHz}$, and $427 \mathrm{GHz}$ with pulse widths 7, 2, $0.6 \mathrm{ps}$, respectively, as shown in Fig. 43(c), could be achieved by actively mode-locked $42.7 \mathrm{GHz}$ Qdash laser by an external RZ $42.7 \mathrm{GHz}$ optical clock signal and a tunable spectral filter.

As noted in this section, optical injection could also be utilized to reduce the phase noise, and hence achieve narrow RF linewidth and low timing jitter in Qdash mode-locked lasers. In continuation, Maldonado-Basilio et al. [316] further investigated the optical locking dynamics on the single section Qdash laser. Alternatively, Sooudi et al. [317] demonstrated a higher intensity $\sim 15 \mathrm{~dB}$ and $\sim 160 \mathrm{kHz}$ linewid th $\mathrm{RF}$ spectrum under $\mathrm{CW}$ injection locking (at negative detuning) compared to the free-running $\sim 20 \mathrm{GHz}$ Qdash InAs/InGaAsP self-mode-locked laser. This was attributed to the supermode noise reduction of the laser which led to amplification of mode-locked peak. In another study, tunable harmonic mode locking via optical injection in Qdash laser to generate microwave pulses with twice the self-mode-locked frequency was demonstrated [318]. Double locking with simultaneous optical injection and optical feedback was investigated by Sooudi et al. and showed two time reduction in the time bandwidth product, and two orders of magnitude reduction in the RF linewidth [319].

\subsubsection{Selected system-level applications}

Because of the small timing jitter, thanks to the low phase noise, InAs/InP Qdash mode-locked laser have found profound interest in many applications requiring narrow mode-beating linewidth like optical-signal processing, optoelectronic oscillators [282, 297, 320], frequency comb generation, WDM system, etc. Here, we briefly review some of these applications, and direct the readers to existing review papers for further information [241, 250].

Truly all optical clock recovery at $40 \mathrm{GHz}$ employing Qdash mode-locked laser was first demonstrated by Renaudier et al. [321, 322] with jitter characteristics in compliant with the ITU recommendations. This was followed by a series of experimental demonstrations of the wavelength tunability [323], all optical frequency down conversion [258], and retiming, reshaping, and reamplifying (3R) generators at $40 \mathrm{Gbps}$ [324]. Clock recovery utilizing different modulation formats such as RZ-OOK [325], NRZ- and RZ-DPSK [325], burst operation [326], as well as subharmonic $40 \mathrm{GHz}$ clock recovery from 40, 80 [327-329], 160 [330, 331], and 320 Gbps [332, 333] has also been demonstrated.

The potential of Qdash mode-locked lasers for millimeter tone generation stems from the self-mode locking capability without any external oscillator and reduced signal linewidth. In addition, a direct modulation of the laser should be possible without assistance from an external modulator [334, 335]. This radio-over-fiber technique was demonstrated on the $60 \mathrm{GHz}$ system for broadband wireless services using 1.5 Gbps OOK and 3.03 Gbps OFDM QPSK modulation schemes by Lecoche et al. [336] with floor free BER measurements and error vector magnitude (EVM) $19 \%$ for a signal-to-noise ratio of $21.5 \mathrm{~dB}$ which is less than the criterion for successful detection (23\%) [250]. The advantages of $60 \mathrm{GHz}$ Qdash mode-locked laser were further assessed by in-door and outdoor 5 Gbps operation experiments and shown an error floor of $10^{-7}$ [337] and further improvement of the EVM to 11\% with high single-to-noise-ratio of $25 \mathrm{~dB}$ [338].

Frequency comb generation is another viable application exploiting the unique features of InAs/InP Qdash mode-locked laser. This has been demonstrated in various reports [254, 339, 340] . Moreover, improvements to noise reduction [341] and stability tests [342] were also recently performed. Besides, the broad lasing multi-longitudinal modes profile of the Qdash laser was itself exploited for multi-channel WDM system demonstration, as discussed in section. 5.1.2.

\subsection{InAs/InP Qdash semiconductor optical amplifie rs}


In As/InP Qdash material which exhibits broad gain profile has been employed as SOA. As seen in In As/InP Qdot SOA, Qdash SOA also showed fast index and gain dynamics, and the capability of amplifying multiple wavelengths without any crosstalk. A systematic review on the advancements of Qdash SOA has been discussed below.

\subsubsection{Broad gain}

Depolyment of multi-stack self-assembled Qdash layers as an active region for SOA was first studied by Bilenca et al. [343] on the In As/InGaAlAs material system. The anti-relection coated $4.5 \times 2100$ $\mu \mathrm{m}^{2}$ device exhibited a broad gain $-3 \mathrm{~dB}$ bandwidth of $>50 \mathrm{~nm}$ centered at $\sim 1.53 \mu \mathrm{m}$. A linear increase in the chip gain with $1.5 \mathrm{~dB}$ uncertainty was observed on increasing the injection current and reaching a maximum $12 \mathrm{~dB}$ at $150 \mathrm{~mA}$. Later, Reithmaier et al. demonstrated a $120 \mathrm{~nm}$ SOA wavelength band exhibiting $>10 \mathrm{~dB}$ chip gain with peak value reaching $25 \mathrm{~dB}$ at $\mathrm{CW}$ current of 300 $\mathrm{mA}$, from a $3.5 \times 2500 \mu \mathrm{m}^{2}$ 4-stack Qdash active region. A large flat amplification characteritics over large input range was observed with a saturation output power of $18 \mathrm{dBm}$ at $0 \mathrm{dBm}$ input power and extracted $3 \mathrm{~dB}$ saturation output power range froms $16 \mathrm{dBm}$ at $1.537 \mu \mathrm{m}$ to $18 \mathrm{dBm}$ at $1.585 \mu \mathrm{m}[26$, 344], as shown in Fig. 44(a). On the InAs/InGaAsP material system, Lelarge et al. [207, 244] reported a $-3 \mathrm{~dB}$ gain bandwidth of $50 \mathrm{~nm}$ and a total amplification bandwidth (gain $>0 \mathrm{~dB}$ ) of 210 $\mathrm{nm}$ from a $1500 \mu \mathrm{m}$ long Qdash SOA with $7^{\circ}$ tilted waveguide and anti-reflection coated. Analysis of the chip gain and noise figure against the injection current for the transverse electric polarization showed an increasing chip gain and reaching a maximum $20 \mathrm{~dB}$ at $300 \mathrm{~mA}$ while the noise figure decreased to $6 \mathrm{~dB}$ which is the lowest value reported on Qdash SOA platform. The performance was further improved with gain up to $30 \mathrm{~dB}$ at $350 \mathrm{~mA} \mathrm{CW}$ current by utilization of an optimized DaWELL strucuture [345]. Looking at the recent encouraging demonstrations of large bandiwdth and peak gain from the chirped Qdash active region structures discussed in section. 4.4, we believe that there is still room of Qdash SOA performance improvement..
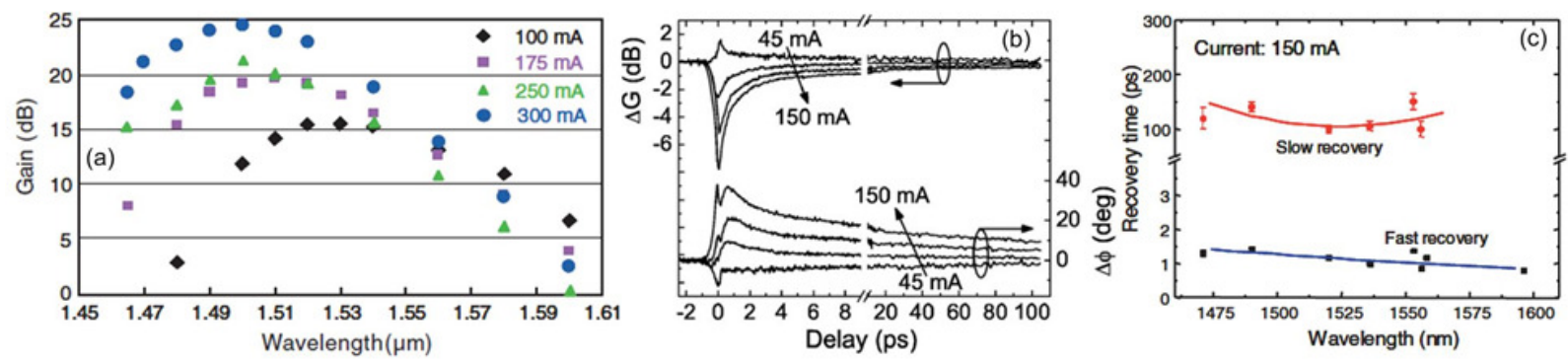

Fig. 44. (a) Optical gain spectra of a $3.5 \times 2500 \mu \mathrm{m}^{2}$ long and Qdash SOA at different drive currents. A maximum chip gain of $25 \mathrm{~dB}$ was obtained at a drive current of $300 \mathrm{~mA}$. (b) Time resolved gain and phase recovery of Qdash SOA for the bias currents of 45, 75, 100, and $150 \mathrm{~mA}$ at a fixed operating wavelength of $1523 \mathrm{~nm}$. (c) Characteristic gain recovery times of Qdash SOA as function of wavelength at a fixed bias of $150 \mathrm{~mA}$. The lines are guides to the eyes. Collected from [26, 346].

\subsubsection{Ultrafast gain and index dynamics}

Gain and phase changes of a weak probe pulse following a strong pump pulse in the InAs/In GaAlAs Qdash SOA was reported by van der Poel et al. [346]. By employing the degenerate heterodyne pump-probe setup (with $150 \mathrm{fs}$ pulse width), the dynamics of the SOA were measured at $1.523 \mu \mathrm{m}$ (gain maximum) probe pulse. As shown in Fig. 44(b), at $150 \mathrm{~mA}$ bias, the SOA reacted very fast with $<1 \mathrm{ps}$ rise time followed by a recovery with two time components due to bi-exponential fit; first being a fast component of $1.6 \mathrm{ps}$ and second a slower component of $130 \mathrm{ps}$. While the former time component was ascribed to the combination of carrier relaxation (high lying dash carrier relaxes into the active states) and carrier capture from barrier to the dash, the latter was attributed to the recovery of the total carrier density of the device, i.e. the effective carrier lifetime. Similar results were also reported by Lunnemann et al. [347] and analogous to the InAs/InP Qdots system discussed in section. 3.3.2. A weak dependence of the gain recovery time on the bias current and the wavelength 
was observed (see Fig. 44(c)), attributed to the dominating phonon scattering and inhomogeneous broadening of the Qdashes. The phase dynamics, on the other hand, showed complex and different characteristics but along the same time scale as the gain recovery except that the fast component of the recovery was smaller relative to the slow component. This was related to the strong effect on the refractive index by changes in the carrier population in Qdash states, wetting layers, and/or barrier region where as only the population of the active states affects the gain $[26,348]$. However, the phase response compared to the Qdots counterpart was much larger which suggested larger LEF in the present case. In fact, Zilkie et al. [166] measured this value to be 5-12 with increasing bias current, on the InAs/InGaAlAs Qdash SOA. Moreover, ultrafast dynamics of 0.1-0.5 ps and 1.2-2.0 ps was found in the case of Qdash SOA on fitting the short lived dynamics with double exponential, and was attributed to the spectral hole burning and carrier heating, respectively [165]. In general, the gain and phase dynamics recovery were in between Qdots and Qwell SOA counterpart, but with a potential of reaching ultra-broad gain profile.

A further investigation on the Qdash SOA dynamics by Capua et al. [349] revealed a nearly instantaneous gain response at energies far above and below the pulse energy on a multi-wavelength pump-probe setup with $150 \mathrm{fs}$ resolution. This was shown by the transmission of probe at $1.57 \mu \mathrm{m}$ and $1.53 \mu \mathrm{m}$ which was $20 \mathrm{~nm}$ away from the gain maximum and was attributed to combination of complex non-linear processes such as two-photon absorption, inhomogeneity and the Qwire-like density of states. The condition to achieve this response was also investigated subsequently [350] and found that only under high optical and electrical excitations this was possible. Moreover, the cross gain saturation response at $1.52 \mu \mathrm{m}$ and $1.6 \mu \mathrm{m}$, with excitation at $1.55 \mu \mathrm{m}$ under low optical and electrical excitation, showed that the recovery at the short wavelength followed the biexponential fit with time constants $0.5 \mathrm{ps}$ and $33 \mathrm{ps}$ while the long wavelength followed single exponential fit of $1.54 \mathrm{ps}$ [351]. This was attributed to the larger role played by the fast interband carrier relaxation, similar to the observation by van der Poel et al. [346]. The noise properties based on coherent spectral hole measurement (since it is caused by the FWM like non linear interration between the signal and the noise) of InAs/InP Qdash nanostructure based SOA was also investigated by Hadaas et al. [344] who predicted a wide coherent spectral hole of about $0.5-1 \mathrm{THz}$, broader by one order of magnitude compared to the Qwell SOA counterpart [5], and later experimentally demonstrated by Capua et al. [352] with value around 500-600 GHz.

\subsubsection{High speed amplification and signal processing}

The unique characteristics of the Qdash SOA which are dictated by the inhomogeneously broadened gain profile and the fast gain and phase recovery dynamics with impressive demonstrations of single and multiple channel amplification and procesing makes them potential cahdidates in future high speed optical communicatio. In addition, the low noise properties of these nanostructures stands out which are crucial in a SOA perofrmance. Here we reviewed some of these achievements.

Single signal processing via wavelength conversion based on FWM on Qdash SOA was investigated by Bilenca et al. [343] who utilized a pump signal fixed at $1.542 \mu \mathrm{m}$ and tunable frequency $( \pm 1000 \mathrm{GHz})$ probe signal. The conversion efficiency of the conjugate FWM product for both the positive and negative detuning was measured and found to be same in shape and was attributed to the reduction in the LEF [353, 354]. A 40 ps probe signal at a repetition rate of 500 $\mathrm{MHz}$ and an average input power of $-7 \mathrm{dBm}$ was detuned from the CW pump by $6.2 \mathrm{~nm}$ with large signal-to-noise ratio. On the other hand, cross gain modulation was demonstrated by $2.5 \mathrm{Gbps} \mathrm{NRZ}$ data at a large detuning of $50 \mathrm{~nm}(7.5 \mathrm{THz})$ with in the inhomogneously broadened gain spectrum of $\sim 1.52 \mu \mathrm{m}$ InAs/InGaAlAs Qdash SOA [355]. The converted pulses possessed high signal-to-noise ratio and the measured BER at the Qdash SOA output of the signals at the original and converted wavelength were identical ( sensitivy $10^{-9}$ at $-26 \mathrm{dBm}$ ) indicative of efficient conversion process. The results are shown in Figs. 45(a) and (b). However, wide cross gain modulation bandwidth could be achieved with the expense of speed due to slow gain recovery (slower carrier escape time at shorter wavelengths due to raised energy levels of wetting layers or barriers at shorter wavelength side) 
[356] and intradash coupling. In other words, the carrier transport time between the spatially separated Qdashes would be too long to follow the faster singals [5].

The cross talk between different wavelengths due to coupling among the Qdash ensembles outside the homogneous linewidth via wetting layers was readily exploited in the ultra-broadband Qdash SOA by simultaneous signal processing and amplification of multiple wavelength channels. For instance, simultaneous amplification of 8 different wavelengths at the same time was performed by Alizon et al. [357] utilizing a single Qdash SOA. Each of the channels were coupled into a fiber, modulated by $10 \mathrm{Gbps}$ PRBS data stream, and then into the SOA. All the input signal powers were at $-20 \mathrm{dBm}$ and after amplification to a maximum of $\sim 0 \mathrm{dBm}$. BER measurements of all the 8 channels, as shown in Fig. 45(c), behaved very similar with no cross talk and penalty after amplification and resembled that of the receiver with sensitivity $10^{-9}$ at $-19 \mathrm{~dB}$ [26]. In addition, high speed transmission experiment by time domain multiplexing four $10 \mathrm{Gbps}$ singals (one $40 \mathrm{Gbps}$ bit stream) was also performed. A clear open eye diagram of the amplified signal with significantly improved quality factor was observed from the low amplification power to deep sturation at $0.5 \mathrm{dBm}$ input power, indicative of the capability of Qdash SOA for signal recovery at high speeds [5, 352].
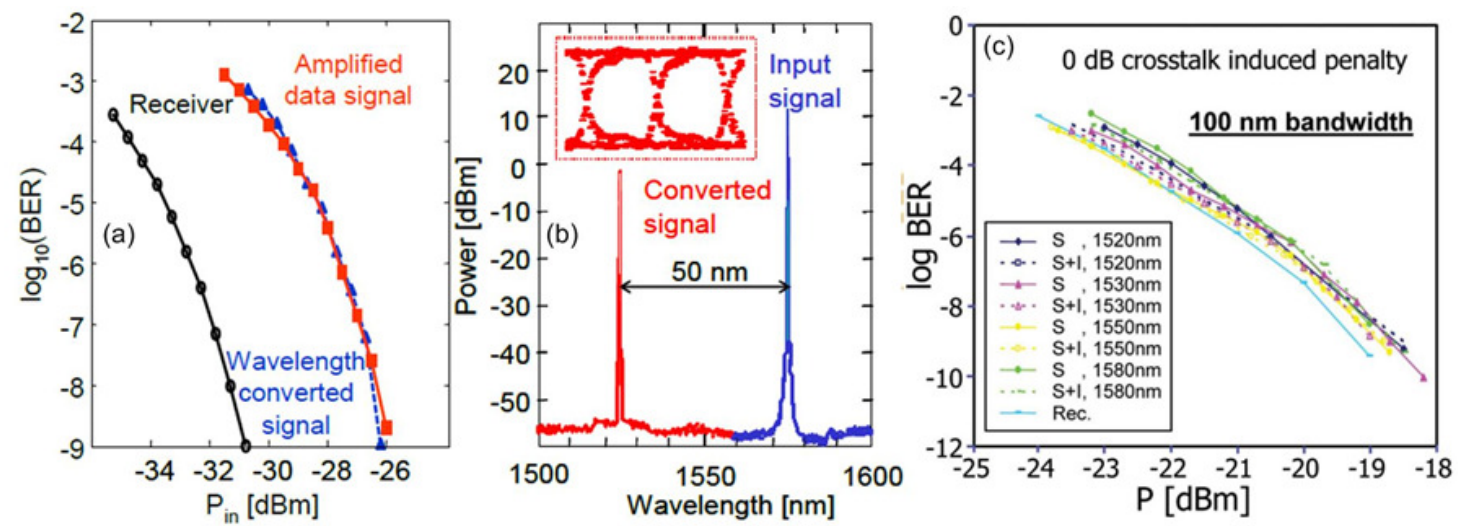

Fig. 45. (a) Cross gain modulation experiments based BER measurements of $2.5 \mathrm{Gbps}$ PRBS data at a $50 \mathrm{~nm}$ detuning. (b) The corresponding spectrum of modulated input signal (right side in blue) and wavelength converted output signal (left side in red). The eye-diagram of a $2.5 \mathrm{Gbps}$ wavelength converted PRBS signal is shown in the inset. (c) BER as a function of the input power for 8 channels simultaneously amplified by Qdash SOA. No cross talk is observed at a data rate of $10 \mathrm{Gbps}$ for each channel. Taken from [5, 358];

A systematic study on the cross saturation dynamics in the InAs/InGaAlAs Qdash SOA was studied by Alizon et al. [359] by amplifying two wavelength data streams at $10 \mathrm{Gbps}$ (signal and inteference) utilizing the optical amplifier and measuring the BER. It was found that for small (large) detuning of $4 \mathrm{~nm}(40 \mathrm{~nm})$, the cross saturation was determined by the dynamics of homogeneously (inhomogen eously) broadened gain region. The large detuning imposed a $2 \mathrm{~dB}$ penalty at a BER $10^{-9}$ for singal wavelength < inteference wavelenth and no penalty in the vice versa case was observed, ascribed to the large cross-saturation effects on the short wavelength side of the gain peak. Additional investigation at low bit rates (2 Gbps) revealed that the cross-saturaton between the channels depends on the detuning, bit rate, and spectral placement. Recently, the versatility of highly inhomogneous Qdash SOA was re-affirmed by Capua et al. [360] who demonstrated simulataneous wavelength conversion with no cross-talk on two independent, spectrally separated by $30 \mathrm{~nm}, 10$ Gbps channel via FWM process and each accompanied by a CW pump. Moreover, the effect of FWM and cross talk were examined by observation of the converted $10 \mathrm{Gbps}$ signal at one channel and measurements of its BER performance in the presence of second channel. A BER of $6 \times 10^{-9}$ at -4 $\mathrm{dBm}$ received power was measured on both the channels indicative of increased optical power requirement for detection.

The potential of In As/InP Qdash SOA for fast light with varible opitcal delays utilizing the FWM process was also demonstrated by Martinez et al. [361] on a microwave frequency modulated signal which underwent group index variation leading to a maximum optical tunable delay up to $136 \mathrm{ps}(\sim 55)$ at $250 \mathrm{MHz}(2 \mathrm{GHz})$ on changing the bias current. This was attributed to the enhanced degenerate FWM efficieny process. 


\subsection{InAs/InP Qdash superluminescent diodes}

Interest in $\mathrm{C}-\mathrm{L}-\mathrm{U}$ band $\mathrm{SLDs}$ has been growing owing to their broader applications in crossdisciplinary fields besides optical communications, as discussed in section 1 and 3.4. Currently, this wavelength window is dominated by the InAsGaAsP/InP Qwell active region platform based diodes which are already commercialized. The drive to attain comparatively flat-top emission and highly efficient devices was rather limited in Qwell active region, and hence paved the way for exploring self-assembled InAs Qdots/Qdashes on InP platform [362]. In this regard, our group was the first to demonstrate room temperature broad emission from InAs/InGaAlAs/InP Qdash SLD spanning 110 $\mathrm{nm}$ bandwidth with output power $\sim 1.5 \mathrm{~mW}$, as shown in Fig. 46(a). The device configuration was based on $50 \times 1000 \mu \mathrm{m}^{2}$ gain section with $1000 \mu \mathrm{m}$ integrated photon absorber. The corresponding emission spectra exhibited $<0.3 \mathrm{~dB}$ spectral ripple, and measured $12.3 \mu \mathrm{m}$ coherence length in air [363]. The latter measurement setup was based on optical fiber coupled Michelson interferometer system. Recently, we investigated the SLD characteristics on the chirped barrier thickness Qdash active region structure under both pulsed and CW operations. High performance SLD characteristics were obtained from both broad area $\left(20 \times 1000 \mu \mathrm{m}^{2}\right)$ and ridge-waveguide $\left(4 \times 2500 \mu \mathrm{m}^{2}\right)$ devices with $1000 \mu \mathrm{m}$ integrated photon absorber, in pulsed operation [362]. An output power more than $20 \mathrm{~mW}$ (Fig. 46(b)) was measured at an emission bandwidth $\sim 82 \mathrm{~nm}$ (Fig. 46(c)) from the former device and corresponding power band width product (PBP) of $1800 \mathrm{~mW}$-nm. The latter ridge-waveguide device exhibited $\sim 12 \mathrm{~mW}$ output power and a maximum bandwidth $\sim 72 \mathrm{~nm}$ (PBP $\sim 905 \mathrm{~mW}-\mathrm{nm}$ ). This investigation showed a significant improvement in the PBP values while maintaining the spectral ripple below $0.3 \mathrm{~dB}$. In the $\mathrm{CW}$ operation, Khan et al. [9] provided a quantitative evidence of simultaneous amplified spontaneous emission from InGaAlAs Qwell and InAs Qdashes from multistack DaWELL structure by demonstrating a record emission bandwidth of $>700 \mathrm{~nm}$ at room temperature from a broad area $50 \times 1000 \mu \mathrm{m}^{2}$ SLD device with output power $>0.3 \mathrm{~mW}$ and capability of reaching beyond $1.3 \mathrm{~mW}$. As shown in Fig. 46(d), the emission wavelength spanned from 1.3$\sim 1.9 \mu \mathrm{m}$ covering the entire $\mathrm{O}-\mathrm{E}-\mathrm{S}-\mathrm{C}-\mathrm{L}-\mathrm{U}$ communication bands. This achievement was attributed to all the possible optical transitions of the Qdash layers and asymmetric Qwells covering the long and short wavelength emission regions, respectively, in addition to junction heating.
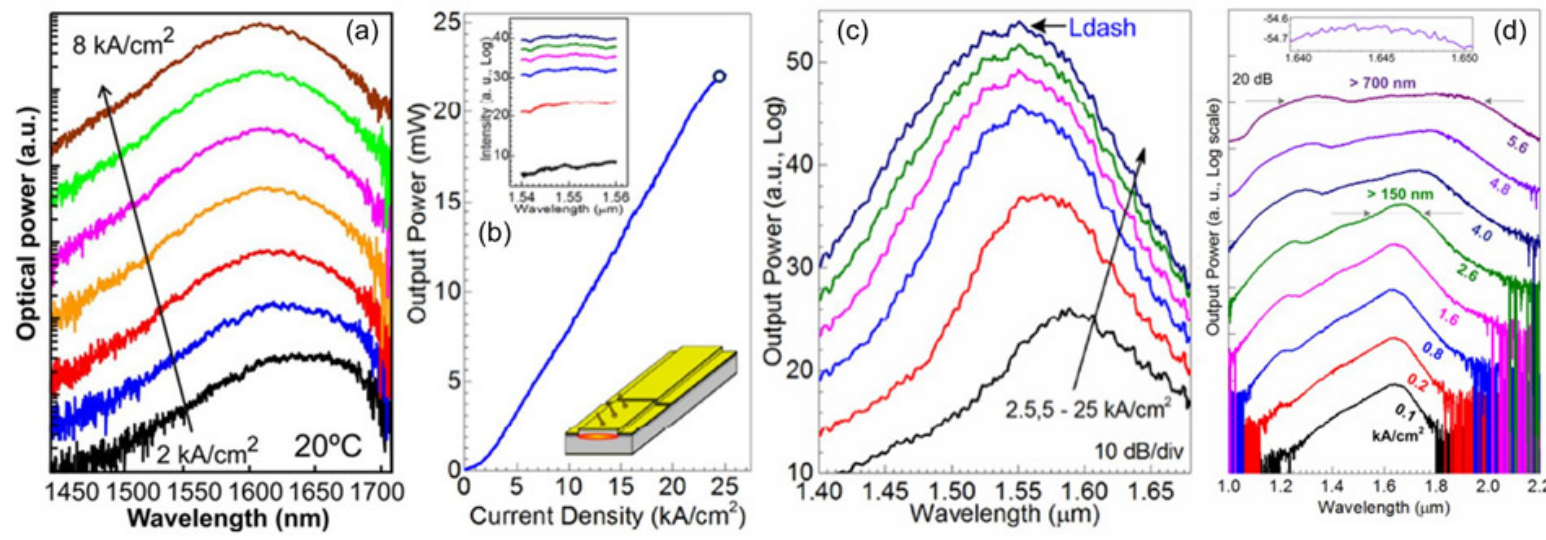

Fig. 46. (a) Broadband spectra from InAs/InGaA 1As DaWELL SLD (1000 $\mu \mathrm{m}$ long gain section with $1000 \mu \mathrm{m}$ photon absorber section) at different current injections from $2-8 \mathrm{kA} / \mathrm{cm}$ measured at $20^{\circ} \mathrm{C}$. (b) Room temperature $L-I$ characteristics of $20 \times 1000 \mu \mathrm{m}^{2}$ chirped Qdash broad area SLD in pulsed operation, and with an integrated $1000 \mu \mathrm{m}$ photon absorber section. (c) The corresponding wideband emission spectra at different injection current density at $2.5 \mathrm{kA} / \mathrm{cm}^{2}$ and $5-25 \mathrm{kA} / \mathrm{cm}^{2}$ in steps of $5 \mathrm{kA} / \mathrm{cm}^{2}$. (d) The ultra-broadband emission spectra at room temperature of the fabricated $50 \times 1000 \mu \mathrm{m}^{2}$ chirped Qdash SLD device at different injection current density under CW operation. The insets in (b) and (d) shows the spectra ripple within $10 \mathrm{~mm}$ from the central wavelength. The emission spectra in (a), (c) and (d) are vertically offset for clarity. Courtesy of [9, 362, 363] 


\section{Conclusion}

In this paper, we reviewed the current advances of InAs/InP based Qdots and Qdash structures from the growth as well as device implementation view point. These 0D and quasi-0D nanostructures show promise in satisfying the exponentially growing internet and mobile connectivity in terms of the bandwidth, power consumption, and cost requirements. The unique properties of Qdot laser exhibiting low threshold, fast modulation, and sub-pico second pulse generation, and Qdash lasers capable of producing broadband stimulated emission and potential to replace multiple discrete laser components in the WDM system by a single device, are capable of meeting the stringint demand of implementing optical interconnects system with energy per bit of $\sim 100 \mathrm{fJ} / \mathrm{bit}$ and eventually $\sim 50$ $\mathrm{fJ} / \mathrm{bit}$ in near future $[364,365]$. In addition, the current bandwidth hungry scenario at $1.55 \mu \mathrm{m}$ telecommunication wavelegnth also calls for extending the transmission system window towards 2 $\mu \mathrm{m}$ wavelength. In this respect, the excellent long wavelength tuning properties of In As Qdot/Qdash laser and related components with their ability to reach this wavelength regime, when implemented according to their strength, pose to realize a green ICT in the near future.

\section{References}

[1] D. Bimberg, M. Grundmann, and N. N. Ledents ov, Quantum dotheterostructures: John Wiley \& Sons, 1999.

[2] A. Zhukov, M. Maksimov, and A. Kovsh, "Device characteristics of long-wavelength lasers based on self-organized quantum dots," Semiconductors, vol. 46, pp. 1225-1250, 2012.

[3] R. Wang, A. Stintz, P. Varangis, T. Newell, H. Li, K. Malloy, et al., "R oom-temperature operation of InAs quantum-dash lasers on InP [001]," IEEE Photonics Technology Letters, vol. 13, pp. 767-769, 2001.

[4] V. Sichkovskyi, M. Waniczek, and J. Reithmaier, 'High-gain wavelength-stabilized $1.55 \mu \mathrm{m}$ InAs/InP (100) based lasers with reduced number of quantum dot active layers," Applied Physics Letters, vol. 102, p. 221117, 2013.

[5] J. P. Reithmaier, G. Eisenste in, and A. Forche1, "InAs/InP quantum-dash lasers and amplifiers," Proceeding s of the IEEE, vol. 95, pp. 1779-1790, 2007.

[6] D. Zhou, R. Piron, M. Dontabactouny, O. Dehaese, F. Grillot, T. Batte, et al., "Low threshold current density of InAs quantum dash laser on InP (100) through optimizing double cap technique," Applied Physics Letters, vol. 94, p. 081107, 2009.

[7] D. Zhou, R. Piron, F. Grillot, O. Dehaese, E. Homeyer, M. Dontabactouny, et al., "Study of the characteristics of $1.55 \mu \mathrm{m}$ quantum dash/dot semic onductor lasers on InP subs trate," Applied Physics Letters, vol. 93, pp. 161104-161104-3, 2008.

[8] M. Z. M. Khan, T. K. Ng, C.-S. Lee, P. Bhattacharya, and B. S. Ooi, "Chirped InAs/InP quantum-dash laser with enhanced broad spectrum of stimulated emission," Applied Physics Letters, vol. 102, p. 091102, 2013.

[9] M. Z. M. Khan, M. A. Majid, T. K. Ng, D. Cha, and B. S. Ooi, "Simultaneous quantum dash-well emission in a chirped dash-in-well superluminescent diode w ith spectral bandwidth> $700 \mathrm{~nm}$," Optics Letters, vol. 38, pp. 3720-3723, 2013.

[10] J. Liu, Z. Lu, P. Poole, P. Barrios, D. Poitras, Z. Jiao, et al., "THz optical pulses from a coupled-cavity quantum-dot laser," Optics Communications, vol. 285, pp. 1323-1325, 2012.

[11] Z. Lu, J. Liu, P. Poole, Z. Jiao, P. Barrios, D. Poitras, et al., "Ultra-high repetition rate InAs/InP quantum dot mode-locked lasers," Optics Communications, vol. 284, pp. 2323-2326, 2011.

[12] Y.-S. Hsieh, Y.-C. Ho, S.-Y. Lee, C.-C. Chuang, J.-c. Tsai, K.-F. Lin, et al., "Dental Optical Coherence Tomography," Sen sors, vol. 13, pp. 89288949, 2013.

[13] S. Ishida and N. N is hizawa, "Quantitative comparison of contrast and imaging depth of ultrahigh-resolution optical coherence tomography images in 800-1700 nm wavelength re gion," Biomedical Optics Exp ress, vol. 3, pp. 282-294, 2012.

[14] V. Kodach, J. Kalkman, D. Faber, and T. Van Leeuwen, "Quantitative comparison of the OCT imaging depth at $1300 \mathrm{~nm}$ and $1600 \mathrm{~nm}$," Biomedical Optics Express, vol. 1, pp. 176-185, 2010.

[15] U. Sharma, E. W. Chang, and S. H. Y un, "Long-wavelength optical coherence tomography at $1.7 \mu \mathrm{m}$ for enhanced imaging depth," Optics Express, vol. 16, pp. 19712-19723, 2008.

[16] K. Scholle, S. Lamrini, P. Koopmann, and P. Fuhrberg, "2 $\mu \mathrm{m}$ laser sources and their pos sible applications," in Frontiers in Guided Wave Optics and Optoelectronics, ed: INTECH, 2010.

[17] D. Stifter, K. Wiesauer, M. Wurm, E. Schlotthauer, J. Kastner, M. Pircher, et al., "Investigation of polymer and polymer/fibre composite materials with opticalcoherence tomography," Measurement Science and Technology, vol. 19, p. 074011, 2008.

[18] H. Tanoto, S. Yoon, T. Ng, C. Ngo, C. Dohrman, E. Fitzgerald, et al., 'Origin and suppression of V-shaped defects in the capping of selfassembled InAs quantum dots on graded Si1-xGex/Si substrate," Applied Physics Letters, vol. 95, p. 2111, 2009.

[19] H. Liu, T. Wang, Q. Jiang, R. Hogg, F. Tutu, F. Pozzi, et al., "Long-wavelength InAs/GaAs quantum-dot laser diode monolithically grown on Ge substrate," Nature Photonics, vol. 5, pp. 416-419, 2011.

[20] J.-X. Fu, S. R. Bank, M. A. Wistey, H. B. Yuen, and J. S. Harris, "Solid-source molecular-beam epitaxy growth of GaInNAsSb/InGaAs single quantum well on InP with photoluminescence peak wavelength at $<$ equation $>2.04<$ span style $=$ "font-size: $.5 \mathrm{em} ; ">\& \mathrm{nbsp} ;</$ span $>\& \# \mathrm{x} 3 \mathrm{bc} ;<$ font face='verdana' $>\mathrm{m}</$ font $><$ /equation $>$," Joumal of Vacuum Science \& Technology B: Microelectronics and Nanometer Structures, vol. 22, pp. $1463-1467,2004$

[21] I. N. Stranski and L. Krastanov, Akad. Wiss. Lit. Mainz Abh. Math. Naturwiss. Kl., Abt. 2B, vol. 146, p. 797, 1938.

[22] L. González, J. M. García, R. García, F. Briones, J. Martínez-Pastor, and C. Ballesteros, "Influence of buffer-layer surface morphology on the self-organize d growth of InAs on InP(001) nanostruc tures," Applied Physics Letters, vol. 76, pp. 1104-1106, 2000.

[23] J. Brault, M. Gendry, G. Grenet, G. Hollinger, Y. Desieres, and T. Benyattou, "Role of buffer surface morphology and alloying effects on the properties of InAs nanostructures grown on InP (001)," Applied Physics Letters, vol. 73, pp. 2932-2934, 1998.

[24] S. Fafard, J. P. McCaffrey, Y. Feng, C. Allen, H. Marchand, L. Isnard, et al., "Toward quantum dot laser diodes emitting at $1.5 \mu \mathrm{m}, "$ in 1998 International Conference on App lication sof Photonic Technology, 1998, pp. 271-276.

[25] J. S. Kim, J. H. Lee, S. U. Hong, H.-S. Kwack, B. S. Choi, and D. K. Oh, "Well-defined excited states of self-assembled InAs/InA 1GaAs quantum dots on InP (001)," Applied Physics Letters, vol. 87, pp. 053102-053102-3, 2005. 
[26] J. Reithmaier, A. Somers, S. Deubert, R. Schwertberger, W. Kaiser, A. Forchel, et al., "InP based lasers and optical amplifiers with wire-/dot-like active regions," Journal of Physics D: Applied Physics, vol. 38, p. 2088, 2005.

[27] H. Saito, K. Nishi, and S. Sugou, "Ground-state lasing at room temperature in long-wavelength InAs quantum-dot lasers on InP (311) B substrates," Applied Physics Letters, vol. 78, pp. 267-269, 2001.

[28] H. Li, J. Wu, Z. Wang, and T. Daniels-Race, "High-density InAs nanowires realized in situ on (100) InP," Applied Physics Letters, vol. 75, pp. $1173-1175,1999$.

[29] R. Nötzel, J. Temmyo, A. Kozen, T. Tamamura, T. Fukui, and H. Hasegawa, "Self-organization of strained GaInAs microstruc tures on InP (311) substrates grown by metalorganic vapor-phase epitaxy," Applied Physics letters, vol. 66, pp. 2525-2527, 1995.

[30] I. Alghoraibi, T. Rohel, N. Bertru, A. Le Corre, A. Letoublon, P. Caroff, et al., "Self-assembled InAs quantum dots grown on InP (311) B substrates: Role of buffer layer and amount of InAs deposited," Jou rnal of Crystal Growth, vol. 293, pp. 263-268, 2006.

[31] P. Caroff, N. Bertru, A. Le Corre, O. Dehaese, T. Rohel, I. Alghoraibi et al., "Achievement of high density InAs quantum dots on InP (311) B substrate emitting at $1.55 \mu \mathrm{m}, "$ Japanese Jou rnalof Applied Physics, vol. 44, p. L1069, 2005.

[32] K. Akahane, N. Ohtani, Y. Okada, and M. Kawabe, "Fabrication of ultra-high density InAs-stacked quantum dots by strain-controlled growth on InP (311) B substrate," Journal of Crystal Growth, vol. 245, pp. 31-36, 2002.

[33] K. Akahane, N. Yamamoto, N. Ohtani, Y. Okada, and M. Kawabe, "Role of Al in spacer layer on the formation of stacked InAs quantum dot structures on InP (311) B," Journal of Crystal Growth, vol. 256, pp. 7-11, 2003

[34] K. Akahane, N. Yamamoto, and T. Kawanishi, "Fabrication of ultra-high-density InAs quantum dots using the strain-compensation technique," Physica Status Solidi (a), vol. 208, pp. 425-428, 2011.

[35] K. Akahane, N. Yamamoto, and M. Tsuchiya, "Highly stacked quantum-dot laser fabricated using a strain compensation technique," Applied Physics Letters, vol. 93, p. 041121, 2008.

[36] J. S. Kim, J. H. Lee, S. U. Hong, W. S. Han, H.-S. Kwack, C. W. Lee, et al., "Formation of self-assembled InAs quantum dots on InAl (Ga) As/InP and effects of a thin GaAs layer," Journal of Crystal Growth, vol. 259, pp. 252-256, 2003.

[37] A. Ponchet, A. Le Corre, H. L'haridon, B. Lambert, and S. Salaün, 'R elationship between self-organization and size of InAs islands on InP (001) grown by gas-source molecular beam epitaxy," Applied Physics Letters, vol. 67, pp. 1850-1852, 1995.

[38] A. Stintz, T. Rotter, and K. Malloy, "Formation of quantum wires and quantum dots on buffer layers grown on InP substrates," Journal of Crystal Growth, vol. 255, pp. 266-272, 2003.

[39] M. Borgstrom, M. Pires, T. Bryllert, S. Landi, W. Seifert, and P. Souza, "InAs quantum dots grown on InAlGaAs lattice matched to InP," Journal of Crystal Growth, vol. 252, pp. 481-485, 2003.

[40] Y. Li, X. Ye, B. Xu, F. Liu, D. Ding, W. Jiang, et al., "Room temperature $1.55 \mu \mathrm{m}$ emission from InAs quantum dots grown on (001) InP substrate by molecular beam epitaxy," Journal of Crystal Growth, vol. 218, pp. 451-454, 2000.

[41] J. S. Kim, J. H. Lee, S. U. Hong, W. S. Han, H.-S. Kwack, and D. K. Oh, "Effects of a thin InGaAs layer on InAs quantum dots embedded in InA 1 (Ga) As," Applied Physics Letters, vol. 83, pp. 3785-3787, 2003.

[42] J. S. Kim, C.-R. Lee, B. S. Choi, H.-S. Kwack, C. W. Lee, E. D. Sim, et al., "Vertical stacks of shape-engineered InA s/InAlGaAs quantum dot and its influences on the lasing characteristics," Applied Physics Letters, vol. 90, pp. 153111-153111-3, 2007.

[43] Y. Yang, B. Jo, J. K im, C.-R. Lee, J. S. Kim, D. K. Oh et al., "Optical stability of shape-engineered InAs/InAlGaAs quantum dots," Journal of Applied Physics, vol. 105, p. 053510, 2009.

[44] Y. Yang, B. Jo, J. Kim, K. J. Lee, M. Ko, C.-R. Lee, et al., "Effects of group-III elements on the growth kinetics of shape-engineered InA s/InAlGaAs quantum dots," Thin Solid Films, vol. 517, pp. 3979-3982, 2009.

[45] C. Gilfert, E.-M. Pavelescu, and J. Reithmaier, "Influence of the As2/As4 growth modes on the formation of quantum dot-like InAs islands grown on InAlGaAs/InP (100)," Applied Physics Letters, vol. 96, p. 191903, 2010.

[46] S. Fafard, Z. Wasilewski, J. McCaffrey, S. Raymond, and S. Charbonneau, "InAs self-assembled quantum dots on InP by molecular beam epitaxy," Applied Physics Letters, vol. 68, pp. 991-993, 1996.

[47] S. Yoon, Y. Moon, T.-W. Lee,E. Yoon, and Y. D. Kim, "Effects of As/P exchange reaction on the formation of InAs/InP quantum dots," Applied Physics Letters, vol. 74, pp. 2029-2031, 1999.

[48] A. Michon, G. Saint-Girons, G. Beaudoin, I. Sagnes, L. Largeau, and G. Patriarche, "InAs/InP (001) quantum dots emitting at $1.55 \mu \mathrm{m}$ grown by low-pressure metalorganic vapor-phase epitaxy," Applied Physics Letters, vol. 87, p. 253114, 2005.

[49] A. Michon, G. Patriarche, G. Beaudoin, G. Saint-Girons, N. Gogneau, and I. Sagnes, "Density of InAs/InP (001) quantum dots grown by metalorganic vapor phase epitaxy: Independent effects of InAs and cap-layer growth rates," Applied Physics Letters, vol. 91, pp. 102107-102107-3, 2007.

[50] R. Nötzel, S. Anantathanasarn, R. P. Van Veldhoven, F. W. Van Otten, T. J. Eijkemans, A. Trampert, et al., "Self assembled InAs/InP quantum dots for te lec om applications in the $1.55 \mu \mathrm{m}$ wave length range: wa velength tuning, stacking, polarization control, and lasing," Japanese Journal of Applied Physics, vol. 45, p. 6544, 2006.

[51] S. Anantathanasarn, R. Nötzel, P. Van Veldhoven, T. Eijkemans, and J. Wolter, "Wavelength-tunable (1.55- $\mu \mathrm{m}$ region) InAs quantum dots in InGaAsP/InP (100) grown by metal-organic vapor-phase epitaxy," Jou mal of Applied Physics, vol. 98, p. 013503, 2005.

[52] R. Notzel, S. Anantathanasarn, P. van Veldhoven, F. van Otten, T. Eijke mans, A. Trampert, et al., "Wa velength tuning of InAs/InP quantum dots: Control of As/P surface exchange reaction," Jou mal of Vacuum Science \& Technology B, vol. 24, pp. 2075-2079, 2006.

[53] S. Anantathanasarn, R. Nötzel, P. Van Veldhoven, F. Van Otten, T. Eijkemans, and J. Wolter, "Stacking and polarization control of wavelengthtunable (1.55 $\mu \mathrm{m}$ region) InAs/InGaAsP/InP (100) quantum dots," Applied Physics Letters, vol. 88, p. 063105, 2006.

[54] S. Anantatha nasarn, R. Nötzel, P. van Veldhoven, F. van Otten, Y. Barbarin, G. Servanton, et al., "Wa velength controlled InAs/InP quantum dots for telec om laser applications," Microelectronics Jou mal, vol. 37, pp. 1461-1467, 2006.

[55] J. Jang, S. Pyun, S. Lee, I. Lee, W. G. Jeong, R. Stevenson, et al., "Room temperature operation of InGaAs/InGaAsP/InP quantum dot lasers," Applied Physics Letters, vol. 85, pp. 3675-3677, 2004.

[56] S. Pyun, S. Lee, I. Lee, H. Kim, W. G. Jeong, J. Jang, et al., "Photoluminescence and lasing characteristics of InGaAs/InGaAsP/InP quantum dots," Journal of Applied Physics, vol. 96, pp. 5766-5770, 2004.

[57] A. Michon, R. Hostein, G. Patriarche, N. Gogneau, G. Beaudoin, A. Beveratos, et al., "Metal organic vapor phase epitaxy of InAsP/InP (001) quantum dots for $1.55 \mu \mathrm{m}$ applic ations: Growth, structural, and optical properties," Journal of Applied Physics, vol. 104, p. 043504, 2008.

[58] P. Poole, J. McCaffrey, R. Williams, J. Lefebvre, and D. Chithrani, "Chemical beam epitaxy growth of self-assembled InAs/InP quantum dots," Jou mal of Vacuum Science \& Technology B, vol. 19, pp. 1467-1470, 2001.

[59] S. Fréchengues, N. Bertru, V. Drouot, B. Lambert, S. Robinet, S. Loualiche, et al., "Wavelength tuning of InAs quantum dots grown on (311) B InP," Applied Physics Letters, vol. 74, pp. 3356-3358, 1999.

[60] P. Poole, R. Williams, J. Lefebvre, J. McCaffrey, and N. Rowell, "Trimming the size of InAs/InP quantum dots grown by CBE," in International Conference on Indium Phosphide and Related Materials, 2000. Conference Proceeding s. 2000 2000, pp. 396-399.

[61] N. N. Ledentsov, V. A. Shchukin, M. Grundmann, N. Kirstaedter, J. Böhrer, O. Schmidt, et al., "Direct formation of vertically coupled quantum dots in Stranski-Krastanow growth," Physical Review B, vol. 54, pp. 8743-8750, 09/15/ 1996.

[62] H. R. Gutiérrez, M. A. Cotta, J. R. R. Bortoleto, and M. M. G. de Carvalho, 'R ole of group V exchange on the shape and size of InA s/InP selfassembled nanostructures," Journal of Applied Physics, vol. 92, pp. 7523-7526, 2002.

[63] Q. Gong, R. Nötzel, P. Van Veldhoven, and J. Wolter, "InAs/InP quantum dots emitting in the wavelength region by inserting ultrathin GaAs and GaP interlayers," Journal of Crystal Growth, vol. 278, pp. 67-71, 2005. 
[64] Q. Gong, R. Nötzel, P. Van Veldhoven, T. Eijkemans, and J. Wolter, "Wavelength tuning of InAs quantum dots grow n on InP (100) by chemicalbeam epitaxy," Applied Physics Letters, vol. 84, pp. 275-277, 2004.

[65] Q. Gong, R. Notzel, P. Van Veldhoven, T. Eijkemans, and J. Wolter, "InAs/InP quantum dots emitting in the $1.55 \mu \mathrm{m}$ wavelength region by inserting submonolayer GaP interlayers," Applied Physics Letters, vol. 85, pp. 1404-1406, 2004.

[66] E. Homeyer, R. Piron, F. Grillot, O. Dehaese, K. Tavernier, E. Macé, et al., "First demonstration of a $1.52 \mu \mathrm{m}$ RT InA s/InP (3 11 1) B laser with an active zone based on a single QD layer," Semiconductor Science and Technology, vol. 22, p. 827, 2007.

[67] Z. R. Wasilewski, S. Fafard, and J. P. McCaffrey, "Size and shape engineering of vertically stacked self-assembled quantum dots," Journal of Crystal Growth, vol. 201-202, pp. 1131-1135, 5// 1999

[68] P. Poole, K. Kaminska, P. Barrios, Z. Lu, and J. Liu, "Growth of InAs/InP-based quantum dots for $1.55 \mu \mathrm{m}$ laser applications," Journal of Crystal Growth, vol. 311, pp. 1482-1486, 2009.

[69] P. Poole, "InP-Based Quantum D ot Lasers," in Advances in Semiconductor La sers. vol. 86, ed, 2012, p. 419.

[70] P. Poole, R. Williams, J. Lefebvre, and S. Moisa, "Using As/P exchange processes to modify InAs/InP quantum dots," Jou mal of Crystal Growth, vol. 257, pp. 89-96, 2003

[71] S. Li, Q. Gong, Y. Lao, K. He, J. Li, Y. Zhang et al., "Room temperature continuous-wave operation of InA s/InP (100) quantum dot lasers grown by gas-source molecular-beam epitaxy," Applied Physics Letters, vol. 93, pp. 111109-111109-3, 2008

[72] G. Elias, A. Létoublon, R. Piron, I. Alghoraibi, A. Nakkar, N. Che valier, et al., "Achievement of high density InAs/GaInAsP quantum dots on mis oriented InP (001) substrates emitting at $1.55 \mu \mathrm{m}, "$ Japanese Jou mal of Applied Physics, vol. 48, p. 070204, 2009.

[73] S. Li, Q. Gong, C. Cao, X. Wang, L. Yue, Q. Liu, et al., "Optical investigation of InAs/InP (100) quantum dots grown by gas source molecular beam epitaxy," Infrared Physics \& Technology, vol 55, pp. 205-209, 2012.

[74] B. Jo, C.-R. Lee, J. S. Kim, W. S. Han, J. H. Song, J.-Y. Leem, et al., "Investigation on the lasing characteristics of InAs/InGaAsP quantum dots with additional confinement structures," Journal of Crystal Growth, vol. 393, pp. 59-63, 2014

[75] G. Elias, A. Letoublon, R. Piron, I. Alghraibi, K. Tavernier, N. Che valier, et al., "Improvement of $1.55 \mu \mathrm{m}$ InAs QD laser using vicinal(001) InP substrate," in IEEE International Conference on Indium Pho sphide \& Related Materials, 2009 (IPRM'09) 2009, pp. 41-44.

[76] C. Paranthon, N. Bertru, O. Dehaese, A. Le Corre, S. Loualiche, B. Lambert, et al., "Height dispersion control of InAs/InP quantum dots emitting at $1.55 \mu \mathrm{m}, "$ Applied Physics Letters, vol. 78, pp. 1751-1753, 2001.

[77] C. Paranthoen, C. Platz, G. Moreau, N. Bertru, O. Dehaese, A. Le Corre, et al., "Grow th and optical characterizations of InAs quantum dots on InP substrate: towards a $1.55 \mu \mathrm{m}$ quantum dot laser," Journalof Crystal Growth, vol. 251, pp. 230-235, 2003.

[78] P. Caroff, N. Bertru, C. Platz, O. Dehaese, A. Le Corre, and S. Loualiche, "Emission wavelength control of InAs quantum dots in a GaInAsP matrix grown on InP (311) B substrates," Joumal of Crystal Growth, vol. 273, pp. 357-362, 2005.

[79] J. Ulloa, P. Koenraad, E. Gapihan, A. Letoublon, and N. Bertru, "D ouble capping of molecular beam epitaxy grown InAs/InP quantum dots studied by cross-sectional scanning tunneling microscopy," Applied Physics Letters, vol. 91, pp. 073106-073106-3, 2007.

[80] S. Fréchengues, V. Drouot, N. Bertru, B. Lambert, S. Loualiche, and A. Le Corre, "Self-organized InAs is lands on InP (311) B substrates emitting around $1.55 \mu \mathrm{m}, "$ Journal of Crystal Growth, vol. 201, pp. 1180-1185, 1999.

[81] A. Nakkar, H. Folliot, A. Le Corre, F. Doré, I. Alghoraibi, C. Labbé, et al., "Optical properties and morphology of InAs/InP (113) B surface quantum dots," Applied Physics Letters, vol. 92, pp. 231911-231911-3, 2008.

[82] N. Bertru, C. Paranthoen, O. Dehaese, H. Folliot, A. Le Corre, R. Piron, et al., "QD haser on InP substrate for $1.55 \mu \mathrm{m}$ emission and beyond," in Proc. of SPIE, 2010, pp. 76081B-76081B-14.

[83] J. F. Girard, C. Dion, P. Desjardins, C. N. Allen, P. J. Poole, and S. Raymond, "Tuning of the electronic properties of self-assembled InAs/InP(001) quantum dots by rapid thermal annealing," Applied Physics Letters, vol. 84, pp. 3382-3384, 2004.

[84] C. K. Chia, S. J. Chua, S. Tripathy, and J. R. Dong, "Group-V intermixing in InAs/nP quantum dots," Applied Physics Letters, vol. 86, pp. -, 2005.

[85] Y. Wang, H. S. Djie, and B. S. Ooi, "Group-III intermixing in InAs/nGaAlAs quantum dots-in-well," Applied Physics Letters, vol. 88, pp. -, 2006.

[86] C. Dion, P. J. Poole, S. Raymond, P. Desjardins, and F. Schiettekatte, "Tuning of the electronic properties of self-assembled InAsInP(001) quantum dots using grown-in defect mediated intermixing," Applied Physics Letters, vol. 89, pp. -, 2006.

[87] S. Barik, H. H. Tan, and C. Jagadish, "Proton implantation-induced intermixing of InAs/nP quantum dots," Applied Physics Letters, vol. 88, pp. -, 2006.

[88] C. Dion, P. Desjardins, M. Chicoine, F. Schiettekatte, P. Poole, and S. Raymond, "Drastic ion-implantation-induced inter-mixing during the annealing of self-assembled InAs/InP (001) quantum dots," Nanotechnology, vol. 18, p. 015404, 2007.

[89] S. Barik, H. H. Tan, and C. Jaga dish, "High temperature rapid thermal annealing of phosphorous ion implanted InAs/nP quantum dots," Applied Physics Letters, vol. 90, pp. -, 2007.

[90] W. Lei and C. Jagadish, "Lasers and photodetectors for mid-infrared 2-3 $\mu \mathrm{m}$ applications," Jou rnal of Applied Physics, vol. 104, pp. -, 2008.

[91] A. Zhukov, V. Ustinov, A. Kovsh, A. Y. Egorov, N. Maleev, N. Ledentsov, et al., "Control of the emission wavelength of self-organized InGaAs quantum dots: main achie vements and present status," Semiconductor science and technology, vol. 14, p. 575, 1999.

[92] Z. Yin and X. Tang, "A review of energy bandgap engineering in III-V semiconductor alloys for mid-infrared laser applications," Solid-State Electronics, vol. 51, pp. 6-15, 1// 2007.

[93] Y. Qiu and D. Uhl, "Self-assembled InAsS b qua ntum dots on (001) InP substrates," Applied Physics Letters, vol. 84, pp. 1510-1512, 2004.

[94] F. Doré, C. Cornet, P. Caroff, A. Ballestar, J. Even, N. Bertru, et al., 'InAs(Sb)/InP(100) quantum dots for mid-infrared emitters: observation of $2.35 \mu \mathrm{m}$ photoluminescence," physica status solidi (c), vol. 3, pp. 3920-3923, 2006.

[95] J. Kotani, P. J. van Veldhoven, and R. Nötzel, "Mid-infrared emission from InAs quantum dots, wells, and dots on well nanostructures grown on InP (100) by metal organic vapor phase epitaxy," Jou mal of Applied Physics, vol. 106, p. 093112, 2009.

[96] Y. Qiu, D. Uhl, R. Chacon, and R. Q. Yang, "Lasing characteristics of InAs quantum-dot hasers on (001) InP substrate," Applied Physics Letters, vol. 83, pp. 1704-1706, 2003.

[97] Y. Qiu, D. Uhl, and S. Keo, "Room-temperature continuous-wave operation of InAsSb quantum-dot lasers near $2 \mu \mathrm{m}$ based on (001) InP substrate," Applied Physics Letters, vol. 84, pp. 263-265, 2004

[98] V. Ustinov, A. Zhukov, A. Y. Egorov, A. Kovsh, S. Zaitsev, N. Y. Gordeev, et al., "Low threshold quantum dot injection hser emitting at 1.9 $\mu \mathrm{m}, "$ Electronics Letters, vol. 34, pp. 670-672, 1998.

[99] V. M. Ustinov, A. R. Kovsh, A. E. Zhukov, A. Y. Egorov, N. N. Ledentsov, A. V. Lunev, et al., "Low-threshold quantum-dot injection heterolaser emitting at $1.84 \mu \mathrm{m}, "$ Technical Physics Letters, vol. 24, pp. 22-23, 1998/01/01 1998.

[100] K. Nishi, M. Yamada, T. Anan, A. Gomyo, and S. Sugou, "Long-wavelength lasing from InAs self-assembled quantum dots on (311) B InP by gas-source molecular beam epitaxy," in IEEE International Conference on Indium Phosphide and Related Materials, 1998, pp. 163-166.

[101] P. Caroff, C. Paranthoen, C. Platz, O. Dehaese, H. Folliot, N. Bertru et al., "High-gain and low-threshold InAs quantum-dot lasers on InP," Applied Physics Letters, vol. 87, pp. 243107-243107-3, 2005.

[102] A. Martinez, K. Merghem, S. Bouchoule, G. Moreau, A. Ramdane, J.-G. Provost, et al., "D ynamic properties of InAs/InP (311) B quantum dot Fabry-Perot lasers emitting at 1.52 $\mu \mathrm{m}, "$ Applied Physics Letters, vol 93, pp. 021101-021101-3, 2008.

[103] K. Nishi, M. Yamada, T. Anan, A. Gomyo, and S. Sugou, "Long-wavelength lasing from InAs self-as sembled quantum dots on (311) B InP," Applied Physics Letters, vol. 73, pp. 526-528, 1998.

[104] C. Paranthoen, N. Bertru, B. Lambert, O. Dehaese, A. Le Corre, J. Even, et al., "Room temperature laser emission of $1.5 \mu \mathrm{m}$ from InAs/InP (311) B quantum dots," Semiconductor Science and Technology, vol. 17, p. L5, 2002 
[105]E. Homeyer, R. Piron, F. Grillot, O. Dehae se, K. Tavernier, E. Macé, et al., "Demonstration of a low threshold current in $1.54 \mu \mathrm{m}$ InAs/InP (311) B quantum dot laser with re duced quantum dot stacks," Japanese Journal of Applied Physics, vol. 46, p. 6903, 2007.

[106] K. Klaime, C. Calò, R. Piron, C. Paranthoen, D. Thiam, T. Batte, et al., "23 and 39 GHz low phase noise monosection InAs/InP (113)B quantum dots mode-locked lasers," Optics Express, vol. 21, pp. 29000-29005, 2013/11/18 2013.

[107] K. Akahane, N. Yamamoto, and T. Kawanishi, "Wavelength tunability of highly stacked quantum dot laser fabricated by a strain compensation technique," in Semiconductor Laser Conference (ISLC), 2010 22nd IEEE International, 2010, pp. 37-38.

[108] K. Akahane, N. Yamamoto, and T. Kawanishi, "The dependence of the characteristic temperature of highly stacked InAs quantum dot laser diodes fabricated using a strain-compensation technique on stacking layer number," in Semiconductor Laser Conference (ISLC), 2012 23rd IEEE International, 2012, pp. 82-83.

[109] I. Alghoraibi, T. Rohel, R. Piron, N. Bertru, C. Paranthoen, G. Elias, et al., "Negative characteristic temperature of long wavelength InAs/AlGaInAs quantum dot lasers grown on InP substrates," Applied Physics Letters, vol. 91, pp. 261 105-261 105-3, 2007.

[110] K. Akahane, N. Yamamoto, H. Sotobayashi, and M. Tsuchiya, "1.7- $\mu \mathrm{m}$ laser emission at room temperature using highly-stacked InAs quantum dots," in Indium Pho sphide and Related Materials, 2008. IPRM 2008. 20 th International Conference on, 2008, pp. 1-3.

[111] J. S. Kim, J. H. Lee, S. U. Hong, W. S. Han, H.-S. Kwack, C. W. Lee, et al., "Long-wavelength laser based on self-assembled InAs quantum dots in InAlGaAs on InP (001)," Applied Physics Letters, vol. 85, pp. 1033-1035, 2004.

[112] J. S. Kim, J. H. Lee, S. U. Hong, W. S. Han, H.-S. Kwack, C. W. Lee, et al., "Room-temperature operation of InP-based InAs quantum dot laser," IEEE Photonics Technology Letters, vol. 16, pp. 1607-1609, 2004.

[113] B. Jo, Y. Yang, J. Kim, M. Ko, K. J. Lee, C.-R. Lee, et al., "Enhancement in the gain of quantum dot laser by increasing overlap integral between electron and hole wave-functions," Thin Solid Films, vol 517, pp. 3983-3986, 2009.

[114] C. Gilfert, V. Ivanov, N. Oehl, M. Yacob, and J. Reithmaier, "High gain $1.55 \mu \mathrm{m}$ diode lasers based on InAs quantum dot like active regions," Applied Physics Letters, vol. 98, pp. 201102-201102-3, 2011.

[115] S. Bhow mick, M. Z. Baten, T. Frost, B. S. Ooi, and P. Bhattacharya, "High Performance InAs/In0. 53Ga0. 23A10. 24As/InP Quantum Dot 1.55 $\mu \mathrm{m}$ TunnelInjection Laser," IEEE Journal of Quantum Electronics, vol. 50, pp. 7-14, 2013.

[116] D. Gready, G. Eisenstein, C. Gilfert, V. Ivanov, and J. P. Reithmaier, "High-Speed Low-Noise InAs/InAlGaAs/InP 1.55-Quantum-Dot Lasers," IEEE Photon ics Technology Letters, vol. 24, pp. 809-811, 2012.

[117]D. Gready, G. Eisenstein, V. Ivanov, C. Gilfert, F. Schnabel, A. Rippien, et al., "High Speed 1.55 um InAs/InGaAlAs/InP Quantum Dot Lasers," IEEE Photonics Technology Letters, vol. 26, pp. 11-13, 2014.

[118]C. N. Allen, P. Poole, P. Marshall, J. Fraser, S. Raymond, and S. Fafard, "InAs self-assembled quantum-dot lasers grown on (100) InP," Applied Physics Letters, vol. 80, pp. 3629-3631, 2002.

[119] C. N. Allen, P. Poole, P. Barrios, P. Marshall, G. Pakulski, S. Raymond et al., "External cavity quantum dot tunable laser through $1.55 \mu \mathrm{m}, "$ Physica E, vol. 26, pp. 372-376, 2005.

[120] J. Renaudier, R. Brenot, B. Dagens, F. Lelarge, B. Rousseau, F. Poingt, et al., "45 GHz self-pulsation with narrow linew idth in quantum dot Fabry-Perot semic onductor lasers at $1.5 \mu \mathrm{m}, "$ Electronics Letters, vol. 41, pp. 1007-1008, 2005.

[121]F. Lelarge, B. Rousseau, B. Dagens, F. Poingt, F. Pommereau, and A. Accard, "Room temperature continuous-wave operation of buried ridge stripe lasers using InAs-InP (100) quantum dots as ac tive core," IEEE Photonics Technology Letters, vol. 17, pp. 1369-1371, 2005.

[122] S. Anantathanasarn, R. Notzel, P. Van Veldhoven, F. Van Otten, Y. Barbarin, G. Servanton, et al., "Lasing of wavelength-tunable (1.55 $\mu \mathrm{m}$ region) InAs/InGaAsP/InP (100) quantum dots grown by metal organic vapor-phase epitaxy," Applied Physics Letters, vol. 89, pp. 073115073115-3, 2006.

[123] S. G. Li, Q. Gong, C. F. Cao, X. Z. Wang, L. Yue, J. Y. Yan et al., "Morphology and shape dependent characteristics of InAs/InP(1\&\#xa0;0\&\#xa 0;0) quantum dot laser grow $\mathrm{n}$ by gas source molecular beam epitaxy," Physica E, vol. 44, pp. 1983-1987, 2012.

[124] S. Li, Q. Gong, Y. Lao, Y. Zhang, S. Feng, and H. Wang, "InA s/InP (100) quantum dot laser w ith high wavelength stability," Electronics Letters vol. 46, pp. 158-159, 2010.

[125]Z. Jiao, Z. Lu, J. Liu, P. Poole, P. Barrios, D. Poitras, et al., "Linew idth enhancement factor of InAs/InP quantum dot lasers around $1.5 \mu \mathrm{m}, "$ Optics Communications, vol. 285, pp. 4372-4375, 2012

[126] H. Kim, W. Jeong, J. Lee, J. Y im, D. Lee, R. Stevenson, et al., "Continuous-wave operation of $1.5 \mu \mathrm{m}$ InGaAs/InGaAsP/InP quantum dot hsers at room temperature," Applied Physics Letters, vol. 87, pp. 083110-083110-3, 2005.

[127] J. Kotani, P. Van Veldhoven, T. De Vries, B. Smalbrugge, E. Bente, M. Smit, et al., "First de monstration of single-layer InAs/InP (100) quantumdot laser: continuous wave, room temperature, ground state," Electronics Letters, vol. 45, pp. 1317-1318, 2009.

[128] J. Kotani, P. J. van Veldhoven, and R. Nötzel, "What Is the Longest Lasing Wavelength of InAs/InP (100) Quantum Dots Grown by Metal Organic Vapor Phase Epitaxy?," Applied Physics Express, vol. 3, p. 072101, 2010.

[129]R. Notzel, "Quantum dots for future nanophotonic devices: hateral ordering, position, and number control," IEEE Photonics Joumal, vol 2, pp. $67-80,2010$.

[130] C. N. Allen, G. Ortner, C. Dion, P. Poole, P. Barrios, J. Lapointe, et al., "Externatcavity quantum-dot laser tunable through $1.55 \mu \mathrm{m}, "$ Applied Physics Letters, vol. 88, pp. 113109-113109-3, 2006.

[131] S. Li, Q. Gong, C. Cao, X. Wang, J. Yan, Y. Wang et al., "A review of external cavity-coupled quantum dot lasers," Optical and Quantum Electronics, vol. 46, pp. 623-640, 2014.

[132] G. Ortner, C. N. Allen, C. Dion, P. Barrios, D. Poitras, D. Dalacu, et al., "External cavity InAs/InP quantum dot laser with a tuning range of 166 nm," Applied Physics Letters, vol. 88, pp. 121119-121119-3, 2006.

[133]P. Chen, Q. Gong, C. Cao, S. Li, Y. Wang, Q. Liu, et al., "High performance external cavity InAs/InP quantum dot lasers," Applied Physics Letters, vol. 98, p. 121102, 2011.

[134] J. Liu, Z. Lu, S. Raymond, P. Poole, P. Barrios, G. Pakulski, et al., "U niform 90-channel multiwavelength InAs/InGaAsP qua ntum dot laser," Electronics Letters, vol. 43, pp. 458-460, 2007.

[135] J. Liu, Z. Lu, S. Raymond, P. Poole, P. Barrios, and D. Poitras, "1.6- $\mu$ m Multiwavelength Emission of an InAs-InGaAsP Quantum-Dot Laser," IEEE Photonics Technology Letters, vol. 20, pp. 81-83, 2008.

[136]Z. Lu, J. Liu, P. Pook, S. Raymond, P. Barrios, D. Poitras, et al., "Low noise InAs/InP quantum dot C-band monolithic multiwavelength lasers for WDM-PONs," in Optical Fiber Communication Conference, 2009, p. JWA27.

[137] J. S. Kim, J. H. Lee, S. U. Hong, H.-S. Kwack, B. S. Choi, and D. K. Oh, "InAs-InAlGaAs quantum dot DFB lasers based on InP [001]," IEEE Photonics Technology Letters, vol. 18, pp. 595-597, 2006.

[138]Z. Lu, P. Poole, J. Liu, P. Barrios, Z. Jiao, G. Pakulki, et al., "High-performance 1.52 um InAs/InP quantum dot distributed feedback laser," Electronics letters, vol. 47, pp. 818-819, 2011.

[139] J. P. Reithmaier, V. Ivanov, V. Sichkovskyi, C. Gilfert, A. Rippien, N. Felgen et al., "InP-based 1.5 \&\#x00B5;m quantum dot lasers: Static and dynamic properties," in IEEE Photonics Conference (IPC), 2013 IEEE, 2013, pp. 1-2.

[140] M. J. Heck, E. A. Bente, B. Smalbrugge, Y.-S. Oei, M. K. S mit, and S. Anantathanasarn, 'Observation of Q-switching and mode-locking in twosection InAs/InP (100) quantum dot lasers around $1.55 \mu \mathrm{m}$," Optics Express, vol. 15, pp. 16292-16301, 2007.

[141] M. J. Heck, A. Renault, E. A. Bente, Y.-S. Oei, M. K. Smit, K. S. Eikema, et al., "Passively mode-locked 4.6 and 10.5 GHz quantum dot laser diodes around $1.55 \mu \mathrm{m}$ with harge operating regime," IEEE Jou mal of Selected Topics in Quantum Electronics, vol. 15, pp. 634-643, 2009.

[142] M. Tahvili, L. Du, M. Heck, R. Nötzel, M. Smit, and E. Bente, "Dual-wavelength passive and hybrid mode-locking of 3, $4.5 \mathrm{and} 10 \mathrm{GHz}$ InAs/InP (100) quantum dot lasers," Optics Express, vol. 20, pp. 8117-8135, 2012. 
[143] M. J. Heck, E. J. Salumbides, A. Renault, E. A. Bente, Y.-S. Oei, M. K. Smit, et al., "Analysis of hybrid mode-locking of two-section quantum dot lasers operating at $1.5 \mu \mathrm{m}, "$ Optics Express, vol. 17, pp. 18063-18075, 2009.

[144]Z. Lu, J. Liu, P. Poole, P. Barrios, D. Poitras, S. Raymond, et al., "A passive mode-locked InAs/InP quantum dot laser with pulse duration of les s than $300 \mathrm{fs}$," in SPIE OPTO: Integrated Optoelectronic Devices, 2009, pp. 722413-722413-7.

[145]Z. Lu, J. Liu, S. Raymond, P. Poole, P. Barrios, and D. Poitras, "312-fs pulse generation from a passive C-band InAs/InP quantum dot modelocked laser," Optics Express, vol. 16, pp. 10835-10840, 2008.

[146] J. Liu, Z. Lu, S. Raymond, P. Poole, P. Barrios, and D. Poitras, "Dual-wavelength 92.5 GHz self-mode-locked InP-based quantum dot laser," Optics Letters, vol. 33, pp. 1702-1704, 2008.

[147]Z. Lu, J. Liu, P. Poole, S. Raymond, P. Barrios, D. Poitras, et al., "An L-band monolithic InAs/InP quantum dot mode-locked laser with femtosecond pulses," Optics Express, vol. 17, pp. 13609-13614, 2009.

[148]Z. Jiao, J. Liu, Z. Lu, X. Zhang, P. J. Poole, P. J. Barrios, et al., "A-Band InAs/InP Quantum Dot Semiconductor Mode-Locked Laser Emitting 403-GHz Repetition Rate Pulses," IEEE Photonics Technology Letters, vol. 23, pp. 543-545, 2011.

[149]Z. Jiao, J. Liu, Z. Lu, X. Zhang, P. J. Poole, P. J. Barrios, et al., "T unable terahertz be at signal generation from an InAs/InP quantum-dot modelocked laser combined with external-cavity," IEEE Photonics Technology Letters, vol. 24, pp. 518-520, 2012.

[150] S. Weiss, J. M. Wiesenfeld, D. S. Chemla, G. Raybon, G. Sucha, M. Wegener, et al., "Carrier capture times in $1.5 \mu$ m multiple quantum well optical amplifiers," Applied Physics Letters, vol. 60, pp. 9-11, 1992.

[151]P. J. A. Thijs, L. F. Tiemeijer, P. I. Kuindersma, J. J. M. Binsma, and T. van Dongen, "High-performance $1.5 \&$ mu;m wavelength InGaAsInGaAsP strained quantum well lasers and amplifiers," Quan tum Electronics, IEEE Jou rnal of, vol. 27, pp. 1426-1439, 1991.

[152] G. Eisenstein, J. M. Wiesenfeld, M. Wegener, G. Sucha, D. S. Chemla, S. Weiss, et al., "Ultrafast ga in dynamics in $1.5 \mu \mathrm{m}$ multiple quantum well optical amplifiers," Applied Physics Letters, vol. 58, pp. 158-160, 1991.

[153] E. Bogaart, R. Notzel, Q. Gong, J. Haverkort, and J. Wolter, "Ultrafast carrier capture at room temperature in InAs/InP quantum dots emitting in the $1.55 \mu \mathrm{m}$ wavelength region," Applied Physics Letters, vol. 86, pp. 173109-173109-3, 2005.

[154] T. Akiyama, M. Ekawa, M. Sugawara, K. Kawaguchi, H. Sudo, A. Kuramata, et al., "An ultrawide-band semic onductor optical amplifier having an extremely high penalty-free output power of $23 \mathrm{dBm}$ achieved with quantum dots," IEEE Photonics Technology Letters, vol. 17, pp. 1614$1616,2005$.

[155] T. Akiyama, M. Ekawa, M. Sugawara, H. Sudo, K. Kawaguchi, A. Kuramata, et al., "An ultraw ide-band (120 nm) semiconductor optical amplifier having an extremely-high penalty-free output power of $23 \mathrm{dBm}$ realized with quantum-dot active layers," in Optical Fiber Communication Conference, 2004, p. PD12.

[156] T. Akiyama, M. Sugawara, and Y. Arakawa, "Quantum-dot semiconductor optical amplifiers," Proceeding s of the IEEE, vol. 95, pp. 1757-1766, 2007.

[157] K. Akahane, N. Yamamoto, T. Umezawa, A. Kanno, and T. Kawanishi, "A semiconductor optical amplifier comprising highly stacked InAs quantum dots fabricated using the strain-compensation technique," Japanese Jou mal of Applied Physics, vol. 53, p. 04EG02, 2014.

[158] A. Matsumoto, Y. Takei, A. Matsushita, K. Akahane, Y. Matsushima, and K. Utaka, "Fundamental characteristic s of 1550nm-band 20-layerstacked QD-SOA grown on InP(311)B substrate for all-optical logic gate de vice," in Microoptics Conference (MOC), 2013 18th, 2013 , pp. 1-2.

[159] N. Kim, J. Oh, M. Kim, D. Lee, S. Pyun, W. Jeong, et al., "Gain characteristics of InAs/InGaAsP quantum dot semiconductor optical amplifiers at $1.5 \mu \mathrm{m}, "$ Applied physics letters, vol. 90, pp. 241108-241108-3, 2007.

[160] G. Contestabile, A. Maruta, and K. Kitayama, "Gain dynamics in quantum-dot semiconductor optical amplifiers at 1550 nm," Photonics Technology Letters, IEEE, vol. 22, pp. 987-989, 2010.

[161] P. Borri, W. Langbein, J. M. Hvam, F. Heinrichsdorff, M. H. Mao, and D. Bimberg, "Ultrafast gain dynamics in InAs-InGaAs quantum-dot amplifiers," Photonics Technology Letters, IEEE, vol. 12, pp. 594-596, 2000.

[162] T. Vallaitis, C. Koos, R. Bonk, W. Freude, M. Laemmlin, C. Meuer, et al., "Slow and fast dynamics of gain and phase in a quantum dot semic onductor optical amplifier," Optics Express, vol. 16, pp. 170-178, 2008/01/07 2008.

[163] K. Solis-Trapala, Y. An, R. Notzel, H. J. Dorren, and R. J. Manning, "Gain and Phase Dynamics of an InAs/InGaAsP/InP Quantum-Dot Semic onductor Optic al Amplifier at 1.55 ?m," in Advanced Photonics \& Renewable Energy, Karlsruhe, 2010, p. NMB2.

[164] A. J. Zilkie, J. Meier, P. W. Smith, M. Mojahedi, J. S. Aitchison, P. J. Poole, et al., "Femtosecond gain and index dynamics in an InAs/InGaAsP quantum dot amplifier operating at 1.55 Ám," Optics express, vol. 14, pp. 11453-11459, 2006.

[165] A. J. Zilkie, J. Meier, M. Mojahe di, P. J. Poole, P. Barrios, D. Poitras, et al., "Carrier dynamics of quantum-dot, quantum-dash, and quantum-well semic onductor optical amplifiers operating at $1.55 \mu \mathrm{m}$, "IEEE Journal of Quantum Electronics, vol. 43, pp. 982-991, 2007.

[166] A. J. Zilkie, J. Meier, M. Mojahe di, A. S. Helmy, P. J. Poole, P. Barrios, et al., "Time-Res olved Linew idth Enhancement Factors in Quantum Dot and Higher-Dimensional Semiconductor Amplifiers Operating at 1.55\$ mu $\{\backslash$ hbox $\{\mathrm{m}\}\}$," Journal of Lightwave Technology, vol. 26, pp. $1498-1509,2008$

[167] J. Park, N. Kim, Y. Jang, E. Lee, J. Lee, J. Baek, et al., "Gain dynamics of an InAs/InGaAsP quantum dot semiconductor optical amplifier operating at $1.5 \mu \mathrm{m}, "$ Applied Physics Letters, vol. 98, p. 011107, 2011.

[168] M. Sugawara, N. Hatori, M. Ishida, H. Ebe, Y. Arakawa, T. Akiyama, et al., "Recent progress in self-assembled quantum-dot optical de vices for optical telecommunication: temperature-insensitive $10 \mathrm{~Gb} \mathrm{~s}-1$ directly modulated lasers and $40 \mathrm{~Gb}$ s- 1 signal-re generative amplifiers," Journal of Physics D: Applied Physics, vol. 38, p. 2126, 2005.

[169] G. Contes tabile, A. Maruta, S. Sekiguchi, K. Morito, M. S ugaw ara, and K. Kitayama, "Regenerative amplification by using self-phase modulation in a quantum-dot SOA," Photonics Technology Letters, IEEE, vol. 22, pp. 492-494, 2010

[170] J. Park, Y. Jang, J. Baek, N. Kim, K. Yee, H. Lee, et al., "Gain recovery in a quantum dot semic onductor optical amplifier and corres ponding pattern effects in amplified optical signals at $1.5 \mu \mathrm{m}, "$ Optics express, vol. 20, pp. 6215-6224, 2012.

[171]Z. Lu, J. Liu, S. Raymond, P. Poole, P. Barrios, D. Poitras, et al., "Highly efficient non-degenerate four-wave mixing process in InAs/InGaAsP quantum dots," Electronics letters, vol. 42, pp. 1112-1114, 2006.

[172] G. Contestabile, Y. Yoshida, A. Maruta, and K. Kitayama, "Coherent Wavelength Conversion in a Quantum Dot SOA," IEEE Photonics Technology Letters, vol. 25, pp. 791-794, 2013.

[173] G. Contestabile, A. Maruta, and K. I. Kitayama, "Four Wave Mixing in Quantum Dot Semiconductor Optical Amplifiers," IEEE Journal of Quan tum Electronics, vol. 50, pp. 379-389, 2014.

[174] G. Contestabile, A. Maruta, S. Sekiguchi, K. Morito, M. Sugawara, and K.-i. Kitayama, "Cross-gain modulation in quantum-dot SOA at 1550 nm," Quan tum Electronics, IEEE Journal of, vol. 46, pp. 1696-1703, 2010.

[175] K. Akahane, N. Yamamoto, and T. Kawanishi, "Broad-B and Electroluminescence from Highly Stacked InAs Quantum Dot at Telec om-Band," Advanced Materials Research, vol. 871, pp. 269-273, 2014.

[176] T. Utzmeier, P. A. Postigo, J. Tamayo, R. Garcia, and F. Briones, "Transition from self-organized InSb quantum-dots to quantum dashes," Applied Physics Letters, vol. 69, pp. 26742676, 1996.

[177] S. Guo, H. Ohno, A. Shen, F. Matsukura, and Y. Ohno, "InAs self-organized quantum dashes grown on GaAs (211) B," Applied Physics Letters, vol. 70, pp. 2738-2740, 1997.

[178]H. Li, Q. Zhuang, X. Kong, Z. Wang, and T. Daniels-Race, "Self-organization of wire-like InAs nanostructures on InP," Journal of Crystal Growth, vol. 205, pp. 613-617, 1999.

[179] J. Brault, M. Gendry, O. Marty, M. Pitaval, J. Olivares, G. Grenet, et al., "Staggered vertical self-organization of stacked InAs/InA 1As quantum wires on InP(001)," Applied Surface Science, vol. 162-163, pp. 584-589, 8/1/ 2000 
[180]R. Schwertberger, D. Gold, J. Reithmaier, and A. Forchel, "Epitaxial growth of $1.55 \mu \mathrm{m}$ emitting InAs quantum dashes on InP-based heterostructures by GS-MBE for long-wavelength laser applications," Jo urnal of Crystal Growth, vol. 251, pp. 248-252, 2003.

[181]R. Schwertberger, D. Gold, J. Reithmaier, and A. Forchel, "Long-wavelength InP-based quantum-dash lasers," IEEE Photonics Technology Letters, vol. 14, pp. 735-737, 2002.

[182] T. Rotter, A. Stintz, and K. Malloy, "InP based quantum dash lasers with $2 \mu \mathrm{m}$ wavelength," in IEE Proceeding s Optoelectronics 2003, pp. 318321.

[183] A. Sauerwald, T. Kummell, G. Bacher, A. Somers, R. Schwertberger, J. Reithmaier, et al., "Size control of InAs quantum dashes," Applied Physics Letters, vol. 86, pp. 253112-253112-3, 2005.

[184] T. Kümmell, A. Sauerwald, A. Somers, A. Löffler, J. Reithmaier, A. Forchel, et al., "Structural and optical analysis of size-controlled InAs quantum dashes," Physica E: Low-dimen sional Systems and Nano structures, vol. 32, pp. 108-110, 2006.

[185] A. Sauerwald, T. Kümmell, A. Löffler, A. Somers, J. Reithmaier, A. Forchel, et al., "Scanning transmission electron microscopy of vertically stacked self organized quantum structures," Physica Status Solidi (c), vol. 3, pp. 3947-3950, 2006.

[186]Z. Mi, J. Y ang, and P. Bhattacharya, "Growth and characteristics of P-doped InAs tunnel injection quantum-dash lasers on InP," IEEE Photonics Technology Letters, vol. 18, pp. 1377-1379, 2006.

[187]P. Podemski, G. Sęk, K. Ryczko, J. Misiewicz, S. Hein, S. Höfling, et al., "Columnar quantum dashes for an active region in polarization

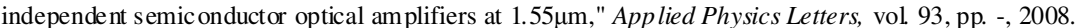

[188]Z. Mi and P. Bhattacharya, "DC and Dynamic Characteristics of P-Doped and Tunnel Injection 1.65-InAs Quantum-Dash Lasers Grown on InP (001)," IEEE Journal of Quan tum Electronics, vol. 42, pp. 1224-1232, 2006.

[189] S. Hein, P. Podemski, G. Sęk, J. Misiew icz, P. Ridha, A. Fiore, et al., "Orientation dependent emission properties of columnar quantum dash laser structures," Applied Physics Letters, vol. 94, p. 241113, 2009.

[190] A. Musiał, P. Podemski, G. Sęk, P. Kaczmarkiewicz, J. Andrzejewski, P. Machnikowski, et al., "Height-driven linear polarization of the surface emission from quantum dashes," Semiconductor Science and Technology, vol. 27, p. 105022, 2012.

[191] A. Ukhanov, R. Wang, T. Rotter, A. Stintz, L. Lester, P. Eliseev, et al., 'Orientation dependence of the optical properties in InAs quantum-dash lasers on InP,"Applied Physics Letters, vol. 81, pp. 981-983, 2002.

[192] D. P. Popescu and K. J. Malloy, "Anisotropy of carrier transport in the active region of lasers with self-assembled InAs quantum dashes," IEEE Photonics Technology Letters, vol. 18, pp. 2401-2403, 2006.

[193] P. Podemski, R. Kudrawiec, J. Misiewicz, A. Somers, R. Schwertberger, J. Reithmaier, et al., "Thermal quenching of photoluminescence from InAs In0. 53Ga 0. 23A10. 24As/InP quantum dashes with different sizes," Applied Physics Letters, vol. 89, p. 151902, 2006.

[194]N. A. Jahan, C. Hermannstädter, J.-H. Huh, H. Sasakura, T. J. Rotter, P. Ahirwar, et al., "Temperature dependent carrier dynamics in telecommunication band InAs quantum dots and dashes grown on InP substrates," Journal of Applied Physics, vol 113, p. 033506, 2013.

[195] W. Rudno-Rudziński, G. Sęk, K. Ryczko, R. Kudrawiec, J. Misiew ic z, A. Somers, et al., "Optically probed wetting layer in InAs/InGaAlAs/InP quantum-dash structures," Applied Physics Letters, vol. 86, p. 101904, 2005.

[196] W. Rudno-Rudziński, R. Kudrawiec, P. Podemski, G. Sęk, J. Misiewicz, A. Somers, et al., "Photoreflectance-probed excited states in InAs/ InGaAlAs quantum das hes grown on InP substrate," Applied Physics Letters, vol. 89, p. 031908, 2006.

[197] W. Rudno-Rudziński, R. Kudraw iec, G. Sęk, J. Misiew icz, A. Somers, R. Schwertberger, et al., "Photoreflectance investigation of InAs quantum dashes embedded in In0.53Ga 0.47AsIn0.53Ga0.23A10.24As quantum well grown on InP substrate," Applied Physics Letters, vol. 88, pp. -, 2006.

[198] P. Podemski, R. Kudrawiec, J. Misiewicz, A. Somers, J. Reithmaier, and A. Forchel, "On the tunnel injection of excitons and free carriers from In0. 53Ga0. 47As $/$ In0. 53Ga 0. 23A10. 24As quantum well to InAs In0. 53Ga0. 23A10. 24As quantum dashes," Applied Physics Letters, vol. 89, p. 061902,2006

[199] G. Sęk, P. Poloczek, P. Podemski, R. Kudraw iec, J. Misiewicz, A. Somers, et al., "Experimentale vidence on quantum well-quantum dash energy transfer in tunnel injection structures for $1.55 \mu \mathrm{m}$ emission," Applied Physics Letters, vol 90, p. 081915, 2007.

[200] I. Marko, S. Sweeney, A. Adams, S. Jin, B. Murdin, R. Schwertberger, et al., "Recombination mechanisms in InAs/InP quantum dash lasers studied using high hydrostatic pressure," PhysicaSstatus Solidi (b), vol. 241, pp. 3427-3431, 2004.

[201] C. Walther, W. Hoerstel, H. Niehus, J. Erxmeyer, and W. Masselink, "Growth, structural, and electrical investigation of self-assembled InAs quantum wires on (001) InP," Journal of Crystal Growth, vol. 209, pp. 572-580, 2000.

[202] B. Salem, J. Olivares, G. Guillot, G. Bremond, J. Brault, C. Monat, et al., "Optical properties of self-assembled InAs quantum islands grown on InP (001) vicinal substrates," Applied Physics Letters, vol. 79, pp. 4435-4437, 2001.

[203] J. Garcia, L. González, M. U. González, J. P. Silveira, Y. González, and F. Briones, "InAs/InP (001) quantum wire formation due to anisotropic stress relaxation: in situ stress measurements," Jou mal of Crystal Growth, vol. 227, pp. 975-979, 2001

[204] M. Gendry, C. Monat, J. Brault, P. Regreny, G. Hollinger, B. Salem, et al., "From large to low height dis persion for self-orga nized InAs quantum sticks emitting at $1.55 \mu \mathrm{m}$ on InP (001)," Jou rnal of Applied Physics, vol. 95, pp. 4761-4766, 2004.

[205]D. Fuster, L. González, Y. González, M. U. González, and J. Martínez-Pastor, "Size and emission wavelength control of InAsł InP quantum wires," Journal of Applied Physics, vol 98, p. 033502, 2005.

[206] D. Fuster, M. U. González, L. González, Y. González, T. Ben, A. Ponce, et al., "Size control of InAs/InP (001) quantum wires by tailoring P/As exchange," Applied Physics Letters, vol. 85, pp. 1424-1426, 2004.

[207]F. Lelarge, B. Dagens, J. Renaudier, R. Brenot, A. Accard, F. van Dijk, et al., "Recent advances on InAs/InP quantum dash based semic onductor lasers and optical amplifiers operating at 1.55 $\mu \mathrm{m}$," IEEE Journal of Selected Topics in Quantum Electron ics, vol. 13, pp. 111-124, 2007.

[208] B. Alén, J. Martínez-Pastor, L. González, J. M. García, S. I. Molina, A. Ponce, et al., "Size-filtering effects by stacking InAs/InP (001) selfassembled quantum wires into multilayers," Physical Review B, vol. 65, p. 241301, 2002.

[209]D. Fuster, M. U. González, L. González, Y. González, T. Ben, A. Ponce, et al., "Stacking of InAs/InP (001) quantum wires studied by in situ stress measurements: role of inhomogeneous stress fields," Applied Physics Letters, vol. 84, pp. 4723-4725, 2004.

[210] A. Lenz, F. Genz, H. Eise le, L. Ivanova, R. Timm, D. Franke, et al., "Formation of InAs/InGaAsP quantum-dashes on InP (001)," Applied Physics Letters, vol. 95, pp. 203105-203105-3, 2009.

[211] M. Faugeron, M. Tran, F. Lelarge, M. Chtioui, Y. Robert, E. Vinet, et al., "High power mode locked quantum dash $1.5 \mu \mathrm{m}$ laser with asymmetricalcladding," in CLEO: Science and Innovations, 2012, p. JW2A. 85.

[212] B. Alén, J. Martınez-Pastor, A. Garcia-Cristobal, L. González, and J. M. Garca, "Optical transitions and excitonic recombination in InAs/InP self-assemble d quantum wires," Applied Physics Letters, vol. 78, pp. 4025-4027, 2001.

[213] B. Salem, T. Benyattou, G. Guillot, C. Bru-Che vallier, G. Bremond, C. Monat, et al., "Strong carrier confinement and evidence for excited states in self-assembled InAs quantum islands grown on InP (001)," Physical Review B, vol. 66, p. 193305, 2002.

[214] S. C. Heck, S. Osborne, S. B. Healy, E. P. O'Reilly, F. Lelarge, F. Poingt, et al., "Experimental and theoretical study of InAs/InGaAsP/InP quantum dash lasers," IEEE Journal of Quantum Electronics, vol. 45, pp. 1508-1516, 2009.

[215] S. Heck, S. Healy, S. Osborne, E. O'Reilly, F. Lelarge, F. Poingt, et al., "An a nalysis of $1.55 \mu \mathrm{m}$ InAs/InP quantum dash lasers," Applied Physics Letters, vol. 92, pp. 251105-251105-3, 2008.

[216]H. S. Djie, Y. Wang, B. S. Ooi, D.-N. Wang, J. Hwang, G. T. Dang et al., "Defect annealing of InAs-InAlGaAs quantum-dash-in-asymmetricwell laser," IEEE Photonics Technology Letters, vol. 18, pp. 2329-2331, 2006.

[217]H. Djie, Y. Wang, B. Ooi, D.-N. Wang, J. Hwang, X.-M. Fang et al., "Wavelength tuning of InAs/InAlGaAs quantum-dash-in-well laser using postgrowth intermixing," Electronics Letters, vol 43, pp. 33-35, 2007.

[218] H. Djie, Y. Wang, D. Negro, and B. Ooi, "Postgrowth band gap trimming of InAs/InA IGaAs quantum-dash laser," Applied Physics Letters, vol. 90, p. 031101, 2007. 
[219]H. S. Djie, Y. Wang, Y.-H. Ding, D.-N. Wang, J. Hwang, X.-M. Fang et al., "Quantum dash intermixing," IEEE Joumal of Selected Topics in Quan tum Electronics, vol. 14, pp. 1239-1249, 2008.

[220] S. Deubert, A. Somers, W. Kaiser, R. Schwertberger, J. Reithmaier, and A. Forchel, "InP-based quantum dash lasers for wide gain bandwidth applications," Jou mal of Crystal Growth, vol. 278, pp. 346-350, 2005.

[221] A. Somers, W. Kaiser, J. Reithmaier, and A. Forchel, "InP-based quantum dash lasers for broadband optical amplification and gas sensing applications," in International Conference on Indium Pho sphide and Related Materials, 2005. , 2005, pp. 56-59.

[222] A. Somers, W. Kaiser, J. Reithmaier, A. Forchel, M. Gioaninni, and I. Montros set, "Optical gain properties of InA s/InAlGaAs/InP quantum dash structures with a spectral gain bandwidth of more than $300 \mathrm{~nm}$, "Applied Physics Letters, vol. 89, pp. 061 107-061107-3, 2006.

[223] M. Z. M. Khan, T. Ng, C.-S. Lee, D. Anjum, D. Cha, P. Bhattacharya, et al., "Distinct lasing operation from chirped InAs/InP quantum-dash laser," IEEE Photonics Journal, vol. 5, pp. 1501308-1501308, 2013.

[224] C. Tan, H. D jie, C. Tan, V. Hongpinyo, Y. Ding, and B. Ooi, 'The effect of multi active junctions on broadband emission from InAs/InGaAlAs quantum-dash structure," in LEOS Annual Meeting Conference Proceeding s, 2009. LEOS'09. IEEE, 2009, pp. 147-148.

[225]M. Z. M. Khan, T. K. Ng, C.-S. Lee, P. Bhattacharya, and B. S. Ooi, "Investigation of Chirped InAs/InGaAlAs/InP Quantum Dash Lasers as Broadband Emitters," IEEE Jo urnal of Qan tum Electronics, vol. 50, pp. 51-61, 2014.

[226] P. Resneau, M. Calligaro, S. Bansropun, O. Parillaud, M. Krakowski, R. Schwertberger, et al., "High-power and low-noise $1.55 \mu \mathrm{m}$ InP-based quantum dash lasers," in SPIE Photonics Europe, 2004, pp. 22-32.

[227] P. Resneau, M. Calligaro, M. Krakowski, H. Liu, M. Hopkinson, A. Somers, et al., "High power and very low noise operation at 1.3 and $1.5 \mu \mathrm{m}$ with quantum dot and quantum dash Fabry-Perot lasers for microwave links," in Optics/Photonics in Security and Defence, 2006, pp. 63990K$63990 \mathrm{~K}-12$.

[228] S. Hein, V. Von Hinten, W. Kaiser, S. Höfling, and A. Forchel, "D ynamic properties of 1.5 um quantum dash lasers on (100) InP," Electronics Letters, vol. 43, pp. 1093-1095, 2007.

[229] S. Hein, V. Von Hinten, S. Höfling, and A. Forchel, "The impact of p-doping on the static and dynamic properties of $1.5 \mu \mathrm{m}$ quantum dash lasers on InP," Applied Physics Letters, vol 92, p. 011120, 2008.

[230] S. Hein, S. Hofling, and A. Forchel, "Modulation Bandwidth and Linewidth Enhancement Factor of High-Speed 1.55-m Quantum-Dash Lasers," IEEE Photon ics Technology Letters, vol. 21, pp. 528-530, 2009.

[231]C. Dziak, A. Moscho, Y. Li, M. Fanto, V. Kovanis, J. Malowicki et al., "Measurement of the linewidth enhancement factor of a 1550-nm injection-locked quantum dash laser," in The 20 th Annual Meeting of the IEEE Lasers and Electro-Optics Society, 2007. LEOS 2007. ,2007, pp. 433-434.

[232] P. Resneau, M. Calligaro, S. Bansropun, O. Parillaud, M. Krakowski, R. Schwertberger, et al., "Long term ageing of $1.55 \mu \mathrm{m}$ InP-based FabryPerot quantum dash lasers," in Conference on Lasers and Electro-Optics Europe, 2005. CLEO/Europe. 2005 2005, p. 95.

[233] G. Moreau, S. Azouigui, D.-Y. Cong, K. Merghem, A. Martinez, G. Patriarche, et al., "Effect of layer stacking and p-type doping on the performance of InAs/InP quantum-dash-in-a-well lasers emitting at 1.55 $\mu \mathrm{m}$," Applied Physics Letters, vol 89, pp. 241123-241123-3, 2006.

[234] S. Azouigui, B. Dagens, F. Lelarge, J. Provost, A. Accard, F. Grillot, et al., "Tolerance to optical feedback of 10-gb/s quantum-dash-based hasers emitting at $1.51 \mu \mathrm{m}, "$ IEEE Photonics Technology Letters, vol. 19, pp. 1181-1183, 2007.

[235] S. Azouigui, B. Kelleher, S. Hegarty, G. Huyet, B. Dagens, F. Lelarge, et al., "Coherence collapse and low-frequency fluctuations in quantumdash based lasers emitting at $1.57 \mu \mathrm{m}, "$ Optics Express, vol 15, pp. 14155-14162, 2007.

[236] G. Moreau, A. Martinez, K. Merghem, S. Guilet, S. Bouchoule, G. Patriarche, et al., "InAs/InP Quantum Dash Based Electro Optic Modulator with Over $70 \mathrm{NM}$ Bandwidth at $1.55 \mu \mathrm{M}, "$ in IEEE 19th International Conference on Indium Phosphide \& Related Materials, 2007. IPRM'07, 2007, pp. 271-273.

[237]F. B. Lelarge, R. Rousseau, B. Martin, F. Poingt, F. LeGouezigou, and O. P. L Le Gouezigou, "Effect of P-doping on temperature and dynamic performances of 1550nm InAs/InP Quantum Dash based lasers," 2009.

[238] B. Dagens, D. Make, F. Lelarge, B. Rous seau, M. Calligaro, M. Carbonnelle, et al., "High Bandwidth Operation of Directly Modulated Laser Based on Quantum-Dash InAs-InP Material at 1.55 m," IEEE Photonics Technology Letters, vol. 20, pp. 903-905, 2008.

[239] F. Lelarge, N. Chimot, B. Rousseau, F. Martin, R. Brenot, and A. Accard, "Chirp optimization of 1550nm InA s/InP Quantum Dash based directly modulated lasers for $10 \mathrm{~Gb} / \mathrm{s} \mathrm{SMF}$ transmission up to $65 \mathrm{Km}$," in International Conference on Indium Phosphide \& Related Materials (IPRM), 20102010 , pp. 1-3.

[240]F. Lelarge, B. Rousseau, F. Martin, F. Poingt, L. Le Gouezigou, O. Le Gouezigou, et al., "Optimization of Tunneling-Injection InAs/InP (100) Quantum Dashes Lasers for High-Speed Optoelectronic Devices," in. IEEE 19 th International Conference on Indium Phosphide \& Related Materials, 2007. IPRM'07, 2007, pp. 274-277.

[241] G. H. Duan, A. Shen, A. Akrout, F. V. Dijk, F. Lelarge, F. Pommereau et al., "High performance InP-based quantum dash semic onductor modelocked lasers for opticalc ommunications," Bell Labs Technical Jou mal, vol. 14, pp. 63-84, 2009.

[242] D. Zhou, R. Piron, M. Dontabactouny, O. Dehaese, F. Grillot, T. Batte, et al., "Low-threshold current density InAs quantum dash hsers on InP (100) grow n by molecular beam epitaxy," Electronics Letters, vol. 45, pp. 50-51, 2009.

[243] D. Zhou, R. Piron, M. Dontabactouny, E. Homeyer, O. Dehaese, T. Batte, et al., "Effect of stack number on the threshold current density and emission wavelength in quantum dash/dot lasers," Physica Status Solidi (c), vol. 6, pp. 2217-2221, 2009.

[244]F. Lelarge, R. Brenot, B. Rousseau, F. Martin, G. Patriarche, F. Poingt, et al., "Recent developments of InP-based quantum dashes for directly modulated lasers and semiconduc tor optical amplifiers," in Integrated Optoelectronic Devices 2008, 2008, pp. 68890L-68890L-20.

[245] K. Merghem, A. Akrout, A. Martinez, G. Aubin, A. Ramdane, F. Lelarge, et al., 'Pulse ge neration at $346 \mathrm{GHz}$ using a passively mode locked quantum-dash-based laser at $1.55 \mu \mathrm{m}, "$ Applied Physics Letters, vol. 94, pp. -, 2009.

[246] K. Merghem, R. Rosales, A. Martinez, G. Patriarche, A. Ramdane, N. Chimot, et al., "Improvement of modal gain of InAs/InP quantum-dash lasers," in Compound Semiconductor Week (CSW/IPRM), 2011 and 23rd International Conference on Indium Phosphide and Related Materials, 2011 , pp. 1-4.

[247]M. Faugeron, F. Lelarge, M. Tran, Y. Robert, E. Vinet, A. Enard, et al., "High Peak Power, Narrow RF Linewidth Asymmetrical Cladding Quantum-Dash Mode-Locked Lasers," IEEE Journal of Selected Topics in Quan tum Electronics, vol. 19, 2013.

[248] P. Resneau, M. Calligaro, B. Rousseau, F. Lelarge, and M. Krakowski, "Low threshold, very low noise, high temperature operation of $1.55 \mu \mathrm{m}$ InP-based Fabry-Perot quantum dashes-in-a-well (DWELL) hsers," in European Conference on Lasers and Electro-Optics, 2007 and the International Quantum Electronics Conference. CLEOE-IQEC 2007. , 2007, pp. 1-1.

[249] A. Akrout, A. Shen, R. Brenot, F. Van Dijk, O. Legouezigou, F. Pommereau et al., "Separate error-free transmission of eight channels at $10 \mathrm{~Gb} / \mathrm{s}$ using comb generation in a quantum-dash-based mode-locked laser," IEEE Photonics Technology Letters, vol. 21, pp. 1746-1748, 2009.

[250] R. Rosales, K. Merghem, A. Martinez, A. Akrout, J.-P. Tourrenc, A. Accard, et al., "InAs/InP Quantum-Dot Passively Mode-Locked Lasers for 1.55- $\mu \mathrm{m}$ A pplications," IEEE Journal of Selected Topics in Quan tum Electron ics, vol. 17, pp. 1292-1301, 2011.

[251] Y. B. M'Sallem, Q. T. Le, L. Bramerie, Q. T. Nguyen, E. Borgne, P. Besnard et al., "Quantum-dash mode-locked laser as a source for 56-Gb/s DQPSK modulation in WDM multicast applic ations," IEEE Photonics Technology Letters, vol. 23, pp. 453-455, 2011.

[252]Q. T. Nguyen, L. Bramerie, G. Girault, O. Vaudel, P. Besnard, J.-C. Simon, et al., "16x2.5 Gbit/s D ownstream Transmission in Colorless WDMPON Based on Injection-Locked Fabry-Perot Laser Diode Using a Single Quantum Dash Mode-Locked Fabry-Perot Laser as Multi-Wavelength Seeding Source," in Optical Fiber Communication Conference and National Fiber Optic Engineers Conference, San Diego, California, 2009, p. OThA3.

[253]R. Rosales, R. Watts, K. Merghem, C. Calò, A. Martinez, A. Accard, et al., "Quantum Dash Mode Locked Lasers as optical comb sources for OFDM superchannels," in European Conference and Exhibition on Optical Communication, 2012, p. Mo. 1. E. 5. 
[254] A. Martinez, C. Calò, R. Rosales, R. Watts, K. Merghem, A. Accard, et al., 'Quantum dot mode locked lasers for coherent frequency comb generation," in SPIE OPTO, 2013, pp. 89930A-89930A-9.

[255] G. Girault, M. Gay, S. Lobo, L. Bramerie, M. Joindot, J.-C. Simon, et al., "Q uantum dash actively modelocked Fabry-Perot laser module demonstrated as part of wavelength tunable RZ trans mitter," Electronics Letters, vol. 44, pp. 873-875, 2008.

[256] M. Costa e Silva, H. Ramanitra, M. Gay, L. Bramerie, S. Lobo, M. Joindot, et al., "Wa velength tunability assessment of a 170 Gbit/s transmitter using a quantum dash Fabry Perot mode-locked laser," ECOC 2009, 2009.

[257] M. Costa e Silva, L. Bramerie, M. Gay, S. Lobo, M. Joindot, and J. C. Simon, "4\&\#x00D7;170 Gbit/s DWDM/OTDM transmission using only one quantum dash Fabry Perot mode-locked laser," in Optical Communication (ECOC), 2010 36th European Conference and Exhibition on, 2010, pp. 1-3.

[258] M. Costa e Silva, A. Lagrost, L. Bramerie, M. Gay, P. Besnard, M. Joindot, et al., "Up to 427 GHz all optical freque ncy down-conversion clock recovery base d on quantum-dash Fabry-Perot mode-locked laser," Joumal of Lightwave Technology, vol. 29, pp. 609-615, 2011.

[259]H. S. Djie, C. L. Tan, B. S. Ooi, J. Hwang, X.-M. Fang, Y. Wu, et al., "Ultrabroad stimulated emission from quantum-dash laser," Applied Physics Letters, vol. 91, pp. 111116-111116-3, 2007.

[260]C. Tan, H. S. Djie, Y. Wang, C. E. Dimas, V. Hongpinyo, Y. H. Ding, et al., "Wavelength tuning and emission width widening of ultrabroad quantum dash interband laser," Applied Physics Letters, vol. 93, p. 111101, 2008.

[261] B. S. Ooi, H. S. Djie, Y. Wang, C.-L. Tan, J. Hwang, X.-M. Fang et al., "Quantum dashes on InP substrate for broadband emitter applications," IEEE Journalof Selected Topics in Quantum Electronics, vol. 14, pp. 1230-1238, 2008.

[262] C. Tan, H. D jie, Y. Wang, C. Dimas, V. Hongpinyo, Y. Ding, et al., "The influe nce of nonequilibrium distribution on room-temperature lasing spectra in quantum-dash lasers," IEEE Photonics Technology Letters, vol. 21, p. 30, 2009.

[263] C. Chen, Y. Wang, H. S. Djie, B. S. Ooi, L. F. Lester, T. L. Koch, et al., "Intrinsic Dynamics of Quantum-Dash Lasers," IEEE Joumal of Selected Topics in Quan tum Electronics, vol. 17, pp. 1167-1174, 2011.

[264] C.-L. Tan, H. S. Djie, and B. S. Ooi, "Novel multiwavelength emitter for WDM transmis sion utilizing broadband quantum-dash laser diode," in Conference on Lasers and Electro-Optics, 2009, p. CTuY 6.

[265] W. Kaiser, M. Legge, A. Somers, S. Deubert, J. Reithmaier, and A. Forchel, "Single mode CW operating InP based quantum dash distributed feedback lasers at 1.5 to $1.9 \mu \mathrm{m}$," in Conference on Lasers and Electro-Optics Europe, 2005. CLEO/Eu rope. 20052005 , p. 96.

[266] L. Bach, W. Kaiser, R. Schwertberger, J. Reithmaier, and A. Forchel, "1.54 um singlemode InP-based Q-dash lasers," Electronics Letters, vol. 39, pp. 985-987, 2003.

[267] W. Kaiser, K. Mathwig, S. Deubert, J. Reithmaier, A. Forchel, O. Parillaud, et al., "S tatic and dynamic properties of laterally coupled DFB lasers based on InAs/InP QDash structures," Electronics Letters, vol. 41, pp. 808-810, 2005.

[268] K. Mathwig, W. Kaiser, A. Somers, J. P. Reithmaier, A. Forchel, K. Ohira, et al., "DFB lasers with deeply etched vertical grating based on InAsInP quantum-dash structures," IEEE Photonics Technology Letters, vol. 19, p. 264, 2007.

[269] W. Zeller, M. Legge, A. Somers, W. Kaiser, J. Koeth, and A. Forche1, "Singlemode emis sion at $2 \mu \mathrm{m}$ wavelength with InP based quantum dash DFB lasers," Electronics Letters, vol. 44, pp. 354-356, 2008.

[270] S. Hein, A. Somers, W. Kaiser, S. Höfling, J. Reithmaier, and A. Forchel, "Singlemode InAs/InP quantum dash distributed feedback lasers emitting in $1.9 \mu \mathrm{m}$ range," Electronics Letters, vol. 44, pp. 527-528, 2008.

[271] Q. Zou, K. Merghem, S. Azouigui, A. Martinez, A. Accard, N. Chimot, et al., "Feedback-resistant p-type doped InAs/InP quantum-dash distributed feedback lasers for isolator-free $10 \mathrm{~Gb} / \mathrm{s}$ transmission at $1.55 \mu \mathrm{m}, "$ Applied Physics Letters, vol. 97, p. $231115,2010$.

[272] N. Chimot, S. Joshi, G. Aubin, K. Merghem, S. Barbet, A. Accard, et al., "1550nm InAs/InP Quantum Dash based directly modulated lasers for next generation passive optical netw ork," in International Conference on Indium Phosphide and Related Materials (IPRM), 2012 2012, pp. 177180.

[273] B. Dagens, D. Make, O. Le Gouezigou, J. Provost, F. Lelarge, A. Accard, et al., "First Demonstration of 10 Gb/s Direct Modulation with a Buried Ridge D istributed Feedback Laser based on Quantum Dash InAs/InP Material at $1.55 \mu \mathrm{m}, "$ in European Conference on Optical Commun ications, 2006. ECOC 2006. , 2006, pp. 1-2.

[274] B. Dagens, D. Make, O. L. Gouezigou, F. Lelarge, B. Rousseau, A. Accard, et al., "10Gb/s Transmission at 1.55 micrometer with Directly Modulated Quantum Dash Laser and Constant Operation Parame ters up to 85 C," ECOC 2007, 2007.

[275] K. Merghem, S. Azouigui, Q. Zou, A. Martinez, N. Chimot, A. Accard, et al., "1.54 $\mu$ m InAs/InP p-type doped quantum dash based DFB lasers for isolator free operation," in 22nd IEEE International Semiconductor Laser Conference (ISLC), 2010 2010, pp. 178-179.

[276] Y. N. Li, N. Kovanis, and L. V Lester, "Modulation response of an injection-locked $1550 \mathrm{~nm}$ quantum dash semiconductor laser," 2007.

[277] N. A. Naderi, M. Pochet, F. Grillot, N. B. Terry, V. Kovanis, and L. F. Lester, "Modeling the injection-locked behavior of a quantum dash semic onductor laser," IEEE Jo urnal of Selected Topics in Quantum Electronics, vol. 15, pp. 563-571, 2009.

[278]L. F. Lester, N. A. Naderi, F. Grillot, R. Raghunathan, and V. Kovanis, "Strong optical injection and the differential gain in a quantum dash laser," Optics Express, vol. 22, pp. 7222-7228, 2014.

[279] L. Lester, N. Terry, A. Moscho, M. Fanto, N. Naderi, Y. Li, et al., "Giant nonlinear gain coefficient of an InAs/AlGaInAs quantum dot laser," in Integ rated Opto electronic Devices 2008,2008, pp. 68890M-68890M-8.

[280] M. Pochet, N. Naderi, N. Terry, V. Kovanis, and L. Lester, "Dynamic behavior of an injection-locked quantum-dash Fabry-Perot laser at zerodetuning," Optics Express, vol. 17, pp. 20623-20630, 2009.

[281] M. C. Pochet, N. A. Naderi, V. Kovanis, and L. F. Lester, "Modeling the dynamic response of an optically-injected nanostructure diode laser," IEEE Journalof Quantum Electronics, vol. 47, pp. 827-833, 2011.

[282] M. Pochet, N. A. Naderi, Y. Li, V. Kovanis, and L. F. Lester, "T unable photonic oscillators using optically injected quantum-dash diode lasers," IEEE Photonics Technology Letters, vol. 22, pp. 763-765, 2010.

[283] S. Azouigui, B. Dagens, F. Lelarge, A. Accard, D. Make, O. Le Gouezigou, et al., "Systematic investigation of InAs/InP quantum-dash based lasers under external optical feedback," Applied Physics Letters, vol. 92, pp. 201106-201106-3, 2008.

[284] S. Azouigui, B. Dagens, F. Lelarge, J. Provost, D. Make, O. Le Gouezigou, et al., "Optical Feedback Tolerance of Quantum-Dot-and QuantumDash-Based Semiconductor Lasers Operating at 1.55 m," IEEE Journal of Selected Topics in Quantum Electronics, vol. 15, pp. 764 773, 2009.

[285]F. Grillot, N. A. Naderi, M. Pochet, C.-Y. Lin, and L. F. Lester, "Variation of the feedback sensitivity in a $1.55 \mu \mathrm{m}$ InAs/InP quantum-dash Fabry-Perot semic onductor laser," Applied Physics Letters, vol. 93, pp. 191108-191108-3, 2008.

[286]F. Grillot, N. A. Naderi, M. Pochet, C.-Y. Lin, P. Besnard, and L. F. Lester, 'Tuning of the critical feedback level in 1.55- $\mu$ m quantum dash semic onductor laser diodes," IET optoelectronics, vol. 3, pp. 242-247, 2009.

[287] C. Gosset, K. Merghem, A. Martinez, G. Moreau, G. Patriarche, G. Aubin, et al., "Subpic osecond pulse ge neration at 134 GHz using a quantumdash-based Fabry-Perot laser emitting at 1.56 $\mu \mathrm{m}$," Applied Physics Letters, vol. 88, pp. 241105-241105-3, 2006.

[288] K. Merghem, R. Rosales, S. Azouigui, A. Akrout, A. Martinez, F. Lelarge, et al., "Low noise performance of passively mode locked quantumdash-based lasers under external optic al feedback," Applied Physics Letters, vol. 95, p. 131111, 2009.

[289] M. Dontabactouny, C. Rosenberg, E. Semenova, D. Larsson, K. Yvind, R. Piron, et al., "10-GHz 1.59- $\mu \mathrm{m}$ quantum dash passively mode-locked two-section lasers," in SPIE Photonics Europe, 2010, pp. 77201A-77201A-10.

[290]R. Rosales, K. Merghem, A. Martinez, A. Accard, F. Lelarge, and A. Ramdane, "High repetition rate two-section InAs/InP quantum-dash passively mode locked lasers," IPRM 2011, 2011.

[291] R. Rosales, K. Merghem, A. Martinez, A. Accard, F. Lelarge, and A. Ramdane, "Two-section InAs/InP Quantum-Dash Passively Mode Locked Lasers," in CLEO: Science and Innovations, 2011, p. CThG2. 
[292] R. Rosales, S. Murdoch, R. Watts, K. Merghem, A. Martine z, F. Lelarge, et al., "H igh performance mode locking characteristics of single section quantum dash lasers," Optics Express, vol. 20, pp. 8649-8657, 2012.

[293] C.-Y. Lin, Y.-C. Xin, Y. Li, F. Chiragh, and L. Lester, "Cavity design and characteristics of monolithic long-wavelength InAs/InP quantum dash pas sively mode-locked lasers," Optics Express, vol. 17, pp. 19739-19748, 2009.

[294] A. Shen, C. Gosset, J. Renaudier, G. Duan, J. Oudar, F. Lelarge, et al., "U ltra-Narrow Mode-Beating Spectral Line-Width of a Passively ModeLocked Quantum Dot Fabry-Perot Laser Diode," in Optical Communications, 2006. ECOC 2006. Eu ropean Conference on, 2006, pp. 1-2.

[295] A. Shen, J.-G. Provost, A. Akrout, B. Rousseau, F. Lelarge, O. Legouezigou, et al., "Low confinement factor quantum dash (QD) mode-locked Fabry-Perot (FP) laser diode for tunable pulse generation," in Optical Fiber Commun ication Conference, 2008, p. OThK 1.

[296]C. Gosset, K. Merghem, A. Martinez, G. Moreau, G. Patriarche, G. Aubin, et al., "Subpicosecond pulse generation at $134 \mathrm{GHz}$ and low radiofrequency spectral linewidth in quantum dash-based Fabry-Perot lasers emitting at 1.5 $\mu \mathrm{m}$, " Electronics Letters, vol. 42, pp. 91-92, 2006.

[297] A. Akrout, A. Shen, A. Enard, G.-H. Duan,F. Lelarge, and A. Ramdane, "Low phase noise all-optical oscillator using quantum dash modelocked laser," Electronics Letters, vol. 46, pp. 73-74, 2010.

[298] J. Tourrenc, A. Akrout, K. Merghem, A. Martinez, F. Lehrge, A. Shen, et al., "Experimental investigation of the timing jitter in self-pulsating quantum-dash lasers operating at 1.55 Ám," Optics Express, vol. 16, pp. 17706-17713, 2008.

[299] S. Latkowski, R. n. Maldonado-Basilio, and P. Landais, "Sub-picosecond pulse generation by 40-GHz passively mode-locked quantum-dash 1mm-long Fabry-PÚrot laser diode," Optics Express, vol. 17, pp. 19166-19172, 2009.

[300]R. Maldonado-Basilio, S. Latkowski, and P. Landais, "720-fs pulse generation with $40 \mathrm{GHz}$ passively-mode locked quantum-dash FabryP\&\#x00E9;rot laser," in Optical Communication, 2009. ECOC 09. 35 th European Conference on, 2009, pp. 1-2.

[301]R. Maldonado-Basilio, J. Parra-Cetina, S. Latkowski, and P. Landais, "Timing-jitter, optical, and mode-beating linew idths analysis on subpicosec ond optical pulses generated by a quantum-dash passively mode-locked semic onductor laser," Optics Letters, vol. 35, pp. 1184-1186, 2010.

[302] R. Maldonado-Basilio, S. Latkowski, F. Surre, and P. Landais, 'Linew idth analysis of 40-GHz passively mode-locked multi-mode semic onductor lasers," Optics Communications, vol. 283, pp. 299-303, 2010.

[303] S. Latkowski, R. Maldonado-Basilio, and P. Landais, "Short pulse generation with $40 \mathrm{GHz}$ passively-mode locked Q-dashed FabryP\&\#x00E9;rot laser," in 11 th International Conference on Transparent Optical Networks, 2009. ICTON '09. , 2009, pp. 1-4.

[304]R. Maldonado-Basilio, S. Latkowski, S. Philippe, and P. Landais, "40 GHz mode-beating with $8 \mathrm{~Hz}$ linew idth and 64 fs timing jitter from a synchronized mode-locked quantum-dash laser diode," Optics Letters, vol 36, pp. 3142-3144, 2011.

[305] C. Calò, H. Schmeckebier, K. Merghem, R. R osales, F. Lelarge, A. Martinez, et al., "Frequency resolved optical gating characterization of sub-ps pulses from single-section InAs/InP quantum dash based mode-locked lasers," Optics Express, vol. 22, pp. 1742-1748, 2014

[306] C. Calo, H. Schmeckebier, K. Merghem, R. Rosales, F. Lelarge, A. Martinez, et al., "Frequency-resolved optic al gating measurements of sub-ps pulses from InAs/InP quantum dash based mode-locked lasers," in Indium Phosphide and Related Materials (IPRM), 2013 International Conference 2013, pp. 1-2.

[307] S. Joshi, C. Calò, N. Chimot, M. Radziunas, R. Arkhipov, S. Barbet, et al., "Quantum dash based single section mode locked lasers for photonic inte grated circuits," Optics Express, vol. 22, pp. 11254-11266, 2014.

[308] S. Joshi, N. Chimot, S. Barbet, A. Accard, and F. Lelarge, "Mode-locked InAs/InP quantum-dash-based DBR laser with monolithically-integrated SOA," in SPIE OPTO, 2014, pp. 90020C-90020C-8.

[309]S. Joshi, N. Chimot, R. Rosales, S. Barbet, A. Accard, A. Ramdane, et al., "Mode locked InAs/InP Quantum dash based DBR Laser monolithically integrated with a semic onductor optical amplifier," in Indium Phosphide and Related Materials (IPRM), 2013 International Conference on, 2013, pp. 1-2.

[310] G.-H. Duan, F. Lelarge, B. Dagens, R. Brenot, A. Accard, A. Shen et al., "InAs/InP based quantum dot mode-locked semic onductor lasers at 1.5 $\mu \mathrm{m}, "$ 2007, pp. $67821 \mathrm{~V}-67821 \mathrm{~V}-6$.

[311]R. Rosales, K. Merghem, A. Martinez, F. Lelarge, A. Accard, and A. Ramdane, 'Timing jitter from the optical spectrum in semic onductor passively mode locked hsers," Optics Express, vol. 20, pp. 9151-9160, 2012/04/09 2012.

[312] S. Murdoch, R. Watts, Y. Xu, R. Maldonado-Basilio, J. Parra-Cetina, S. Latkowski, et al., "Spectral amplitude and phase measurement of a 40 GHz free-running quantum-dash modelocked laser diode," Optics Express, vol. 19, pp. 13628-13635, 2011.

[313]E. Martin, R. Watts, L. Bramerie, A. Shen, H. Gariah, F. Blache, et al., "Terahertz-bandwidth coherence measurements of a quantum dash laser in passive and active mode-locking operation," Optics Letters, vol. 37, pp. 4967-4969, 2012/12/01 2012.

[314]C. Gosset, K. Merghem, G. Moreau, A. Martinez, G. Aubin, J. L. Oudar, et al., "Phase-amplitude characterization of a high-repetition-rate quantum dash passively mode-locked laser," Optics Letters, vol. 31, pp. 1848-1850, 2006/06/15 2006.

[315] A. Akrout, A. Shen, F. Lelarge, F. Pommereau, H. Gariah, F. B lache, et al., "Spectrum filtering and pulse compression of Quantum-Dash modelocked lasers emitting at $1.55 \mu \mathrm{m}, "$ in 34 th European Conference on Optical Commun ication, 2008. ECOC 2008., 2008, pp. 1-2.

[316] R. Maldonado-Basilio, J. Parra-Cetina, S. Latkowski, N. Calabretta, and P. Landais, "Experimental Investigation of the Optical Injection Locking Dynamics in Single-Section Quantum-Dash Fabry-Pérot Laser Diode for Packet-Based Clock Recovery Applications," Joumal of Lightwave Technology, vol. 31, pp. 860-865, 2013.

[317]E. Sooudi, G. Huyet, J. G. McInerney, F. Lelarge, K. Merghem, R. Rosales, et al., "Injection-locking properties of InAs/InP-based mode-locked quantum-dash lasers at $21 \mathrm{GHz}$," IEEE Photonics Technolog y Letters, vol. 23, pp. 1544-1546, 2011.

[318]E. Sooudi, G. Huyet, J. G. McInerney, F. Lelarge, K. Merghem, A. Martinez, et al., "Observation of harmonic-mode-locking in a mode-locked InA s/InP-based quantum-dash laser with cw optical injection," IEEE Photonics Technolog y Letters, vol. 23, pp. 549-551, 2011.

[319]E. Sooudi, C. de Dios, J. G. McInerney, H. Huyet, L. Lelarge, K. Merghem, et al., "A Novel Scheme for Two-Level Stabilization of Semic onductor Mode-Locked Lasers Using Simultaneous Optical Injection and Optical Feedback," IEEE Joumal of Selected Topics in Quan tum Electronics, vol. 19, pp. 1101208-1101208, 2013.

[320]F. Van Dijk, A. Enard, X. Buet, F. Lelarge, and G.-H. Duan, "Phase noise reduction of a quantum dash mode-locked laser in a millimeter-wave coupled opto-electronic oscillator," Lightwave Technology, Jou mal of, vol. 26, pp. 2789-2794, 2008.

[321] J. Renaudier, B. Lavigne, P. Gallion, and G.-H. Duan, "Study of phase-noise properties and timing jitter of 40-GHz all-optical clock recovery using self-pulsating semic onductor lasers," Jo urnal of lightwave technology, vol. 24, pp. 3734-3742, 2006.

[322] J. Renaudier, B. Lavigne, F. Lelarge, M. Jourdran, B. Dagens, O. Legoue zigou, et al., "Standard-compliant jitter transfer function of all-optical clock recovery at $40 \mathrm{GHz}$ based on a quantum-dot self-pulsating semic onductor laser," Photonics Technology Letters, IEEE, vol. 18, pp. 1249$1251,2006$.

[323] J. Parra-Cetina, S. Latkowski, R. Maldonado-Basilio, and P. Landais, "Wavelength tunability of all-optical clock-recovery based on quantumdash mode-locked laser diode under injection of a 40-Gb/s NRZ data stream," Photonics Technology Letters, IEEE, vol. 23, pp. 531-533, 2011.

[324] X. Tang, S. H. Chung, J. C. Cartledge, A. Shen, A. Akrout, and G.-H. Duan, "Application of a passively mode-locked quantum-dot Fabry-Perot laser in $40 \mathrm{~Gb} / \mathrm{s}$ all-optical 3R regeneration," Optics Express, vol. 18, pp. 9378-9383, 2010.

[325] X. Tang, J. C. Cartle dge, A. Shen, F. van Dijk, A. Akrout, and G.-H. Duan, "Characterization of all-optical clock recovery for $40 \mathrm{~Gb} / \mathrm{s}$ RZ-OOK and RZ-DPSK data using mode-locked semic onductor lasers," Journal of Lightwave Technology, vol. 27, pp. 4603-4609, 2009.

[326] J. Luo, J. Parra-Cetina, P. Landais, H. J. Dorren, and N. Calabretta, "Performance Assessment of 40 G Burst Optical Clock Recovery based on Quantum Dash La ser," IEEE Photonics Technology Letters, vol. 25, pp. 2221-2224, 20132013.

[327] J. Parra-Cetina, J. Luo, N. Calabretta, S. Latkowski, H. J. Dorren, and P. Landa is, "Subharmonic All-Optical Clock Recovery of up to $320 \mathrm{~Gb} / \mathrm{s}$ Signal U sing a Quantum Dash Fabry-Pérot Mode-Locked Laser," Jou mal of Lightwave Technology, vol. 31, pp. 3127-3134, 2013. 
[328] R. Maldonado-Basilio, S. Latkowski, S. Philippe, and P. Landais, "Experimental investigation of harmonic and subharmonic synchronization of $40 \mathrm{GHz}$ mode-locked quantum-dash laser diodes," Optics letters, vol. 36, pp. 1569-1571, 2011.

[329] V. Ronc in, A. O'Hare, S. Lobo, E. Jacquette, L. Bramerie, P. Rochard, et al., "Multi-data-rate system performance of a 40-GHz all-optical clock recovery based on a quantum-dot Fabry-Pérot laser," Photon ics Technology Letters, IEEE, vol. 19, pp. 1409-1411, 2007.

[330] M. Yañez and J. C. Cartledge, "Single harmonically driven electroabsorption modulator for OTDM demultiplexing," Joumal of Lightwave Technology, vol. 29, pp. 1437-1444, 2011.

[331] J. Luo, J. Parra-Cetina, S. Latkowski, R. Maldonado-Basilio, P. Landais, H. Dorren, et al., "Quantum Dash Mode-Locked Laser based Open-Loop Optical Clock Recovery for $160 \mathrm{~Gb} / \mathrm{s}$ Transmission System," in Optical Fiber Communication Conference/National Fiber Optic Eng ineers Conference 2013, Anaheim, California, 2013, p. OTh4D.6.

[332] N. Calabretta, J. Luo, J. Parra-Cetina, S. Latkowski, R. Maldonado-Basilio, P. Landais, et al., "320 Gb/s all-optical clock recovery and time demultiplexing enabled by a single Quantum Dash Mode-Locked Laser Fabry-Perot Optical Clock Pulse Generator," in Optical Fiber Communication Conference/National Fiber Optic Engineers Conference 2013, Anaheim, California, 2013, p. OTh4D.5.

[333] J. Luo, N. Calabretta, J. Parra-Cetina, S. Latkowski, R. Maldonado-Basilio, P. Landais, et al., "320 Gb/s all-optical clock recovery and time demultiplexing after transmission enabled by single quantum dash mode-locked laser," Optics letters, vol. 38, pp. 4805-4808, 2013.

[334] A. Stöhr, A. Akrout, R. B uß, B. Charbonnier, F. van Dijk, A. Enard, et al., "60 GHz radio-over-fiber technologies for broadband wireless service s [Invited]," Journal of Optical Networking, vol. 8, pp. 471-487, 2009.

[335] A. Stohr, S. Babiel, P. J. Cannard, B. Charbonnier, F. Van Dijk, S. Fedderwitz et al., "Millimeter-wave photonic components for broadband wireless systems," Microwave Theory and Techniques, IEEE Transactions on, vol. 58, pp. 3071-3082, 2010.

[336]F. Lecoche, E. Tanguy, B. Charbonnier, H. Li, F. Van Dijk, A. Enard et al., "Transmission quality measurement of two types of 60 GHz millimeter-wave generation and distribution systems," Jou rnal of Lightwave Technology, vol. 27, pp. 5469-5474, 2009.

[337]F. van Dijk, B. Charbonnier, S. Constant, A. Enard, S. Fedderwitz, S. Formont, et al., "Quantum dash mode-locked lasers for millimeter wave signal generation and transmission," in 2010 23RD ANNUAL MEETING OF THE IEEE PHOTONICS SOCIETY, 2010, pp. 187-188.

[338] R. Rosales, B. Charbonnier, K. Merghem, F. Van Dijk, F. Lelarge, A. Martinez, et al., "InAs/InP quantum dash based mode locked lasers for 60 GHz radio over fiber applications," in In dium Pho sphide and Related Materials (IPRM), 2012 International Conference on,2012, pp. 185-187.

[339]F. Van Dijk, M. Faugeron, F. Lelarge, M. Tran, M. Chtioui, Y. Robert, et al., "Asymmetrical Cladding quantum dash Mode-Locked Laser for Terahertz wide frequency comb," in Microwave Photonics (MWP), 2012 International Topical Meeting on, 2012, pp. 282-285.

[340]R. Watts, R. Rosales, S. Murdoch, F. Lelarge, A. Ramdane, and L. Barry, "QDash semic onductor mode-locked lasers as compact subchannel comb for optical OFDM superchannel systems," in CLEO: Science and Innovations, 2012, p. CM1I. 5.

[341] E. Sooudi, S. Sygletos, A. D. Ellis, G. Huyet, J. G. McInerney, F. Lelarge, et al., "Optical frequency comb generation using dual-mode injectionlocking of quantum-dash mode-locked lasers: Properties and applications," IEEE Jou mal of Quan tum Electronics vol. 48, pp. 1327-1338, 2012.

[342] K. Merghem, C. Calo, R. Rosales, X. Lafos se, G. Aubin, A. Martinez, et al., "Stability of Optical Frequency Comb Generated With InAs/InP Quantum-Dash-Based Passive Mode-Locked Lasers," IEEE Journal of Qantum Electronics, vol. 50, pp. 275-280, 20142014.

[343] A. Bilenca, R. Alizon, V. Mikhelashvili, G. Eisenstein, R. Schwertberger, D. Gold, et al., "InAs/InP $1550 \mathrm{~nm}$ quantum dash semic onductor optical amplifiers," Electronics Letters, vol. 38, pp. 1350-1351, 2002

[344] D. Hadass, A. Bilenca, R. Alizon, H. Dery, V. Mikhelashvili, G. Eisens tein et al., "Gain and noise saturation of w ide-band InAs-InP quantum dash optical amplifiers: model and experiments," IEEE Journal of Selected Topics in Quantum Electronics, vol. 11, pp. 1015-1026, 2005.

[345]F. Pommereau, R. Brenot, B. Rous seau, F. Martin, F. Poingt, L. LeGouezigou, et al., "Optimization of 1550nm InAs/InP Quantum Dash and Quantum Dot based semiconductor optical amplifier," in IEEE International Conference on Indium Phosphide \& Related Materials, 2009. IPRM'09. , 2009, pp. 339-342.

[346] M. Van der Poel, J. Mørk, A. Somers, A. Forchel, J. Reithmaier, and G. Eisenstein, "Ultrafast gain and index dynamics of quantum dash structures emitting at 1.55 $\mu \mathrm{m}, "$ Applied Physics Letters, vol. 89, p. 081102, 2006.

[347] P. Lunnemann, S. Ek, K. Yvind, R. Piron, and J. Mørk, "Nonlinear carrier dynamics in a quantum dash optical amplifier," New Journal of Physics, vol. 14, p. 013042, 2012.

[348] M. van der Pol, T. W. Berg, J. Mørk, D. B irkedal, V. Mikhelashvili, G. Eisenstein, et al., "Measurements of gain and index dynamics in quantum dash semiconductor optical amplifiers," in Optical Amplifiers and Their Application s/Integ rated Photonics Research, San Francisco, California, 2004, p. OTuD4.

[349] A. Capua, G. Eisenstein, and J. Reithmaier, "A nearly instantaneous gain response in quantum dash based optical amplifiers," Applied Physics Letters, vol. 97 , p. $131108,2010$.

[350] A. Capua, G. Eisenstein, and J. Reithmaier, "Ultrafast cross saturation dynamics in inhomogeneously broadened InAs/InP quantum dash optical amplifiers," Applied Physics Letters, vol. 98, p. 101108, 2011.

[351]A. Capua, A. Saal, O. Karni, G. Eisenstein, J. P. Reithmaier, and K. Yvind, "Complex characterization of short-pulse propagation through InAs/InP quantum-dash optical amplifiers: from the quasi-linear to the two-photon-dominated regime," Optics Express, vol. 20, pp. 347-353, 2012.

[352] A. Capua, V. Mikhelashvili, G. Eisenstein, J. Reithmaier, A. Somers, A. Forchel, et al., "Direct observation of the coherent spectral hole in the noise spectrum of a saturated InAs/InP quantum da sh amplifier operating near $1550 \mathrm{~nm}$," Optics Express, vol 16, pp. 2141-2146, 2008.

[353] D. Hadass, V. Mikhelashvili, G. Eisenstein, A. Somers, S. Deubert, W. Kaiser, et al., "Time-re solved chirp in an InAs/InP quantum-dash optical amplifier operating with $10 \mathrm{Gbit} / \mathrm{s}$ data," Applied Physics Letters, vol. 87, pp. 021104-021104-3, 2005.

[354] R. Brenot, M. Manzanedo, J.-G. Provost, O. Le gouezigou, F. Pommereau, F. Poingt, et al., "Chirp reduction in quantum dot-like semic onductor optical amplifiers, "ECOC 2007, 2007.

[355] A. Bilenca, R. Alizon, V. Mikhelashhvili, D. Dahan, G. Eisenstein, R. Schwertberger, et al., "Broad-band wavelength conversion ba sed on crossgain modulation and four-wave mixing in InAs-InP quantum-dash semiconductor optical amplifiers operating at $1550 \mathrm{~nm}$," IEEE Photonics Technology Letters, vol. 15, pp. 563-565, 2003.

[356] R. Alizon, A. B ilenca, H. Dery, V. Mikhelashvili, G. Eisenstein, R. Schwertberger, et al., 'Cross-gain modulation in inhomogeneously broadened gain spectra of InP-Based $1550 \mathrm{~nm}$ quantum dash optical amplifiers: Small-signal bandw idth dependence on wavelength detuning," Applied Physics Letters, vol. 82, pp. 4660-4662, 2003.

[357] R. Alizon, D. Hadass, V. Mikhelashvili, G. Eisenstein, R. Schwertberger, A. Somers, et al., "Multiple wa velength amplification in wide band high power $1550 \mathrm{~nm}$ quantum dash optical amplifier," Electronics Letters, vol. 40, pp. 760-761, 2004.

[358] G. Eisenstein, "Do Quantum Dots or Quantum Wire Based Devices Offer a Practical Advantage in Producing Semic onductor Optical Amplifiers over Conventional 2-D Active Media," in Optical Fiber Communication and the National Fiber Optic Engineers Conference, 2007. OFC/NFOEC 2007. Conference on, 2007, pp. 1-2.

[359] R. Alizon, D. Hadass, V. Mikhelashvili, G. Eisenstein, R. Schwertberger, A. S omers, et al., "Cross-saturation dynamics in InAs/InP quantum dash optical amplifiers operating at $1550 \mathrm{~nm}, "$ Electronics Letters, vol. 41, pp. 266-268, 2005.

[360] A. Capua, S. O' duill, V. Mikhelashvili, G. Eisenstein, J. Reithmaier, A. Somers, et al., "Cros s talk free multi channel processing of 10 Gbit/s data via four wave mixing in a $1550 \mathrm{~nm}$ InAs/InP quantum dash amplifier," Optics Express, vol. 16, pp. 19072-19077, 2008.

[361] A. Martinez, G. Aubin, F. Lelarge, R. Brenot, J. Landreau, and A. Ramdane, "Variable optical delays at $1.55 \mu \mathrm{m}$ using fast light in an InAs/InP quantum dash based semiconductor optical amplifier," Applied Physics Letters, vol. 93, pp. 091116-091116-3, 2008.

[362] M. Z. M. Khan, T. K. Ng, and B. S. Ooi, "High Performance 1.55 um Superluminescent Diode Based on Broad Gain InAs/InGaAlAs/InP Quantum Dash Active Region," IEEE Photonics Journal, vol. 6, p. 1600108, 2014. 
[363] C. E. Dimas, C.-L. Tan, H. S. Djie, A. D. McAulay, and B.-S. Ooi, "A temporal coherence study of quantum-dot/dash broadband lasers and superluminescent diodes," IEEE Photon ics Technology Letters, vol. 21, pp. 694-696, 2009.

[364]D. A. Miller, "Device requirements for optical interc onnec ts to silic on chips," Proceedings of the IEEE, vol. 97, pp. 1166-1185, 2009.

[365]E. Timurdogan, C. M. Sorace-Agaskar, J. Sun, E. S. Hos se ini, A. Biberman, and M. R. Watts, "A one femtojoule athermal silicon modulator," arXiv prep rint arXiv:1312.2683, 2013. 